\title{
BASIC CONCEPTS OF ENRICHED CATEGORY THEORY
}

\author{
G.M. KELLY, F.A.A. \\ PROFESSOR OF PURE MATHEMATICS, UNIVERSITY OF SYDNEY
}

Received by the editors 2004-10-30.

Transmitted by Steve Lack, Ross Street and RJ Wood. Reprint published on 2005-04-23.

Several typographical errors corrected 2012-05-13.

2000 Mathematics Subject Classification: 18-02, 18D10, 18D20.

Key words and phrases: enriched categories, monoidal categories.

Originally published by Cambridge University Press, London Mathematical Society Lecture

Notes Series 64, 1982

(C) G.M. Kelly, 2005. Permission to copy for private use granted. 
ACKNOWLEDGEMENTS FOR THE REPRINT:

From THE AUTHOR:

I want first to express my very deep gratitude to the volunteer typists whose efforts have allowed the production of this attractive reprint of 'Basic Concepts of Enriched Category Theory'. They are: Richard Garner, who had begun to re-type the book for his own use and offered several chapters, along with Toby Bartels, William Boshuck, Maria Manuel Clementino, Robert Dawson, Steve Lack, Tom Leinster, Francisco Marmolejo, Shane O'Conchuir, Craig Pastro, Mark Weber and Ralph Wojtowicz - each of whom handled several sections. Thanks also to the many other colleagues who offered to help with the typing.

I am in addition particularly indebted to Donovan Van Osdol for his careful proofreading of the text; to Michael Barr for his coordination of the volunteer effort, for his elegant typesetting of the diagrams, and for his fine work on the index; and to Bob Rosebrugh, the Managing Editor, for his constant encouragement and for many kindnesses: to these, too, I offer my very sincere thanks.

G. Max Kelly

Sydney, April 2005

\section{FROM THE EDITORS:}

It is a pleasure to add this book to the Reprints in Theory and Applications of Categories. There have been requests for its inclusion since we began the series.

As we did for Jon Beck's thesis, we asked for volunteers to retype the text and were again overwhelmed by the response - nearly 30 colleagues volunteered within a day of sending the request. We warmly thank everyone who volunteered and especially those we asked to do the work.

Serendipitously, Richard Garner had begun to re-type the book for his own use and kindly offered his excellent work - much of the first three chapters. We then selected other volunteers to type several sections each. The typists we thank, in addition to Richard Garner, are: Toby Bartels, William Boshuck, Maria Manuel Clementino, Robert Dawson, Steve Lack, Tom Leinster, Francisco Marmolejo, Shane O'Conchuir, Craig Pastro, Mark Weber and Ralph Wojtowicz. Everyone did their part promptly and followed instructions that simplified coordination.

We also thank Donovan Van Osdol for providing a superb proof-reading job and Michael Barr for coordinating the many different files, for rendering the diagrams beautifully using his own diagxy front end for xy-pic, and for the difficult task of creating the index.

The few typographical errors from the original of which the author was aware, and a few noted by the typists, have been corrected without comment.

The Editors of Theory and Applications of Categories 
To my wife and children 


\section{Contents}

$\begin{array}{ll}\text { Introduction } & 1\end{array}$

1 The elementary notions $\quad 7$

1.1 Monoidal categories . . . . . . . . . . . . . . . . . . 7

1.2 The 2-category $\mathcal{V}$-CAT for a monoidal $\mathcal{V} \ldots \ldots \ldots$

1.3 The 2-functor ()$_{0}: \mathcal{V}$-CAT $\rightarrow$ CAT . . . . . . . . . . . . . . 10

1.4 Symmetric monoidal categories: tensor product and duality . . . . . . . . . 11

1.5 Closed and biclosed monoidal categories . . . . . . . . . . . . . . 13

$1.6 \mathcal{V}$ as a $\mathcal{V}$-category; representable $\mathcal{V}$-functors . . . . . . . . . . . . . . 15

1.7 Extraordinary $\mathcal{V}$-naturality . . . . . . . . . . . . . . 17

1.8 The $\mathcal{V}$-naturality of the canonical maps . . . . . . . . . . . . . . . . . . . 19

1.9 The (weak) Yoneda lemma for $\mathcal{V}$-CAT . . . . . . . . . . . . . . . 21

1.10 Representability of $\mathcal{V}$-functors; $\mathcal{V}$-functor of the passive variables . . . . . . 22

1.11 Adjunctions and equivalences in $\mathcal{V}$-CAT . . . . . . . . . . . . . 23

2 Functor categories $\quad 27$

2.1 Ends in $\mathcal{V} \ldots \ldots \ldots \ldots \ldots \ldots$

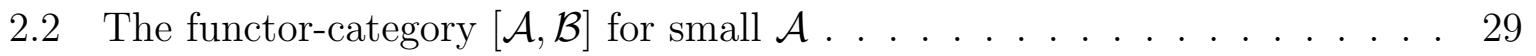

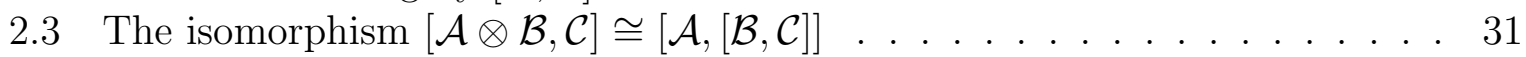

2.4 The (strong) Yoneda lemma for $\mathcal{V}$-CAT; the Yoneda embedding . . . . . . 33

2.5 The free $\mathcal{V}$-category on a Set-category . . . . . . . . . . . . . . 35

2.6 Universe-enlargement $\mathcal{V} \subset \mathcal{V}^{\prime} ;[\mathcal{A}, \mathcal{B}]$ as a $\mathcal{V}^{\prime}$-category . . . . . . . . . . . 35

3 Indexed limits and colimits 37

3.1 Indexing types; limits and colimits; Yoneda isomorphisms . . . . . . . . . . 37

3.2 Preservation of limits and colimits . . . . . . . . . . . . . . . . . 39

3.3 Limits in functor categories; double limits and iterated limits . . . . . . . . 40

3.4 The connexion with classical conical limits when $\mathcal{V}=$ Set . . . . . . . . . . 42

3.5 Full subcategories and limits; the closure of a full subcategory . . . . . . . 44

3.6 Strongly generating functors . . . . . . . . . . . . . . . . 46

3.7 Tensor and cotensor products . . . . . . . . . . . . . . . . . . 48

3.8 Conical limits in a $\mathcal{V}$-category . . . . . . . . . . . . . . . . . . . . 49

3.9 The inadequacy of conical limits . . . . . . . . . . . . . . . . . . . 50

3.10 Ends and coends in a general $\mathcal{V}$-category; completeness . . . . . . . . . . . 52

3.11 The existence of a limit-preserving universe-enlargement $\mathcal{V} \subset \mathcal{V}^{\prime} \ldots \ldots 4$ 
3.12 The existence of a limit- and colimit-preserving universe-enlargement $\mathcal{V} \subset \mathcal{V}^{\prime} 56$

4 Kan extensions $\quad 59$

4.1 The definition of Kan extensions; their expressibility by limits and colimits 59

4.2 Elementary properties and examples . . . . . . . . . . . . . 61

4.3 A universal property of $\operatorname{Lan}_{K} G$; its inadequacy as a definition . . . . . . . 64

4.4 Iterated Kan extensions; Kan adjoints; $\left[\mathcal{A}^{\mathrm{op}}, \mathcal{V}\right]$ as the free cocompletion . . 66

4.5 Initial diagrams as the left Kan extensions into $\mathcal{V}$. . . . . . . . . . . . 68

4.6 Filtered categories when $\mathcal{V}=$ Set . . . . . . . . . . . . . 71

4.7 Factorization into an initial functor and a discrete op-fibration . . . . . . . 73

4.8 The general representability and adjoint-functor theorems . . . . . . . . . . 75

4.9 Representability and adjoint-functor theorems when $\mathcal{V}=$ Set . . . . . . . . 77

4.10 Existence and characterization of the left Kan extension . . . . . . . . . 80

5 Density $\quad 85$

5.1 Definition of density, and equivalent formulations . . . . . . . . . . 85

5.2 Composability and cancellability properties of dense functors . . . . . . . 87

5.3 Examples of density; comparison with strong generation . . . . . . . . . . 89

5.4 Density of a fully-faithful $K$ in terms of $K$-absolute colimits . . . . . . . . 92

5.5 Functor categories; small projectives; Morita equivalence . . . . . . . . . . 94

5.6 Left Kan extensions and adjoint functor theorems involving density . . . . 96

5.7 The free completion of $\mathcal{A}$ with respect to . . . . . . . . . . . . . . 98

5.8 Cauchy completion as the idempotent-splitting completion when $\mathcal{V}=$ Set . 100

5.9 The finite-colimit completion when $\mathcal{V}=$ Set $\ldots \ldots$. . . . . . . . . 101

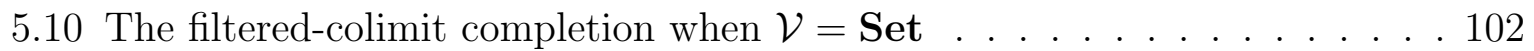

5.11 The image under $[K, 1]:[\mathcal{C}, \mathcal{B}] \longrightarrow[\mathcal{A}, \mathcal{B}] \ldots \ldots$. . . . . . . . . . . . 104

5.12 Description of the image in terms of $K$-comodels . . . . . . . . . . . . . 106

5.13 A dense $K: \mathcal{A} \longrightarrow \mathcal{C}$ as an essentially-algebraic theory . . . . . . . . . . 109

5.14 The image under $[K, 1]:[\mathcal{C}, \mathcal{B}] \rightarrow[\mathcal{A}, \mathcal{B}] \ldots \ldots$. . . . . . . . . 110

6 Essentially-algebraic theories defined by reguli and by sketches 113

6.1 Locally-bounded categories; the local boundedness of $\mathcal{V}$. . . . . . . . . . . 113

6.2 The reflectivity in $\left[\mathcal{A}^{\mathrm{op}}, \mathcal{V}\right]$ of the category of algebras for a regulus . . . . 115

6.3 The category of algebras for a sketch . . . . . . . . . . . . . . 118

6.4 The $\mathcal{F}$-theory generated by a small sketch . . . . . . . . . . . . . . . . . . 122

6.5 Symmetric monoidal closed structure on $\mathcal{F}$-cocomplete categories . . . . . 124

$\begin{array}{ll}\text { Bibliography } & 125\end{array}$

$\begin{array}{ll}\text { Index } & 131\end{array}$ 


\section{Introduction}

(i) Although numerous contributions from divers authors, over the past fifteen years or so, have brought enriched category theory to a developed state, there is still no connected account of the theory, or even of a substantial part of it. As the applications of the theory continue to expand - some recent examples are given below - the lack of such an account is the more acutely felt.

The present book is designed to supply the want in part, by giving a fairly complete treatment of the limited area to which the title refers. The basic concepts of category theory certainly include the notion of functor-category, of limit and colimit, of Kan extension, and of density; with their applications to completions, perhaps including those relative completions given by categories of algebras for limit-defined theories. If we read " $\mathcal{V}$-category" for "category" here, this is essentially the list of our chapter-headings below, after the first chapter introducing $\mathcal{V}$-categories.

In fact our scope is wider than this might suggest; for what we give is also a selfcontained account of basic category theory as described above, assuming as prior knowledge only the most elementary categorical concepts, and treating the ordinary and enriched cases together from Chapter 3 on.

(ii) In order to include the enriched case we begin in Chapter 1 by introducing symmetric monoidal closed categories $\mathcal{V}$, examining their elementary properties, and defining the 2-category $\mathcal{V}$-CAT of $\mathcal{V}$-categories, $\mathcal{V}$-functors, and $\mathcal{V}$-natural transformations, together with the forgetful 2 -functor $(-)_{0}: \mathcal{V}$-CAT $\longrightarrow$ CAT; this much is easy and brief. Next, developing the basic structure of $\mathcal{V}$-CAT - tensor products of $\mathcal{V}$-categories, $\mathcal{V}$-functors of two variables, extraordinary $\mathcal{V}$-natural transformations, $\mathcal{V}$ itself as a $\mathcal{V}$-category, representable $\mathcal{V}$-functors, and the Yoneda lemma - requires verifications of diagram commutativity, whose analogues for $\mathcal{V}=$ Set reduce to fairly trivial equations between functions. This seems to be an inevitable cost of the extra generality; but we have been at pains so to arrange the account that the reader should find the burden a light one. With this done, the discussion of representability, adjunction, and equivalences in $\mathcal{V}$-CAT, which closes Chapter 1, is simple and direct.

The short Chapter 2 takes up the closed structure of $\mathcal{V}$-CAT, given by the $\mathcal{V}$-functorcategory $[\mathcal{B}, \mathcal{C}]$. Since the hom $[\mathcal{B}, \mathcal{C}](T, S)$ is to be an object of $\mathcal{V}$ and not merely a set, more work is once again required than in the $\mathcal{V}=$ Set case. However this hom for small $\mathcal{B}$ is quite a simple limit in $\mathcal{V}_{0}$ - now supposed complete - of the special kind called an end, precisely adapted to the extraordinary $\mathcal{V}$-natural transformations; and from this definition flow easily the desired properties.

The indexed limits and colimits of Chapter 3, along with their various special cases, 
are constructs of the greatest importance even when $\mathcal{V}=$ Set; and the relations between double and iterated indexed limits express a rich content. This importance is scarcely lessened by their reducibility when $\mathcal{V}=$ Set to classical limits - any more than the importance of these classical ones is lessened by their reducibility in good cases to products and equalizers. Even in the case $\mathcal{V}=\mathbf{A b}$ of additive categories, the indexed limits although here they exist when all the classical ones do - are no longer directly reducible to the latter; while for a general $\mathcal{V}$ the indexed limits are essential, and the classical ones no longer suffice. Chapter 3 ends by showing how to expand $\mathcal{V}$ into a bigger universe, without disturbing limits and colimits, so as to allow the free use of functor-categories $[\mathcal{B}, \mathcal{C}]$ with large $\mathcal{B}$.

The remaining chapters 4, 5, and 6, dealing respectively with Kan extensions, density, and algebras defined by limits (or more generally by "topologies"), make use of these limitand colimit-notions to complete the development of our chosen area of basic category theory. Most of the results apply equally to categories and to $\mathcal{V}$-categories, without a word's being changed in the statement or the proof; so that scarcely a word would be saved if we restricted ourselves to ordinary categories alone. Certainly this requires proofs adapted to the case of a general $\mathcal{V}$; but these almost always turn out to be the best proofs in the classical case $\mathcal{V}=$ Set as well. This reflects the hopes with which Eilenberg and the author set out when writing [26], and Lawvere's observation in [54] that the relevant segment of classical logic "applies directly to structures valued in an arbitrary [symmetric monoidal] closed category $\mathcal{V}$ ".

Because of the special properties of Set, there are of course certain results peculiar to the case $\mathcal{V}=$ Set; and we accordingly devote an occasional section to this case alone. Some examples are the commutativity in Set of filtered colimits with finite limits; the notions of initial functor and of discrete op-fibration; and the classical adjoint-functor theorems. We treat all of these for completeness - partly to keep the account self-contained, and partly to compare them, where appropriate, with analogues for a general $\mathcal{V}$.

The little prior knowledge that we do assume is easily available - for instance from [60] - and to have included it here, with examples to enliven it, would have involved an unjustifiable increase in the length. For Chapter 1, it consists of the basic notions of category, functor and natural transformation, including functors of two or more variables and contravariant functors; representable functors and the Yoneda lemma; adjunction and equivalence; and what is meant by a faithful or a fully-faithful functor. From Chapter 2 on, we also need the classical notions of limit and colimit; with the names of such special limits as product, equalizer, pullback, and terminal object; and the meaning of monomorphism and epimorphism. In the rare places where more is assumed but not expounded here, references are given.

(iii) We now turn to what we have omitted from the present book; the list includes many important notions, well meriting an extended treatment, whose inclusion would however have disturbed the essential simplicity of an initial account such as this.

First (to return to the title), the basic concepts of category theory concern categories in our case, $\mathcal{V}$-categories - as the objects of discussion. These form, or live in, a 2-category $\mathcal{V}$-CAT; but 2-categories are not yet the formal objects of discussion, any more than categories are when we study group theory. Category theory casts light on group theory, 
as does 2-category theory on category theory. Hence the step to this next level, where weaker notions of natural transformation naturally arise, is an important one; but it is quite properly deferred pending experience of some particular 2-categories, and we do not take it here. We make a start on some aspects of it in the forthcoming [46].

Closely connected to this is our decision not to discuss the "change of basecategory" given by a symmetric monoidal functor $\mathcal{V} \longrightarrow \mathcal{V}^{\prime}$, and the induced 2functor $\mathcal{V}$-CAT $\longrightarrow \mathcal{V}^{\prime}$-CAT. We do, as we said, consider the forgetful 2-functor $(-)_{0}: \mathcal{V}$-CAT $\longrightarrow$ CAT induced by the canonical $\mathcal{V} \longrightarrow$ Set; but this is entirely elementary, not involving even the definition of a symmetric monoidal functor. The general change of base, important though it is, is yet logically secondary to the basic $\mathcal{V}$-category theory it acts on. To treat it properly needs a careful analysis of the 2-category of symmetric monoidal categories, symmetric monoidal functors, and symmetric monoidal natural transformations - including adjunctions therein and the dual concept of opmonoidal functor. There is evidence in [43] that this itself is best studied in the more general context of categories with essentially-algebraic structure, which draws on the matter of this book together with [45] and [46].

Since the natural setting for the important work of Day ([12], [14], [16]) on the construction of symmetric monoidal closed categories as functor-categories, or as reflective subcategories of these, involves the 2-category of symmetric monoidal categories, this too has been omitted.

One thing we particularly regret omitting is the theory of monads; it could certainly be seen as basic category theory, yet there was no convenient place to put it, and it would have required an extra chapter to itself. Luckily, besides the account of Mac Lane [60] in the classical case, we have the articles of Linton [57], Dubuc [22], and Kock [51], covering various aspects of the enriched case. We also have the elegant 2-categorical treatment by Street [70], which provides some argument for deferring the topic until 2-categories have been more closely studied. A consequence is our failure to discuss the completeness and cocompleteness of the 2 -category $\mathcal{V}$-Cat of small $\mathcal{V}$-categories - which is most easily referred back to the completeness and the cocompleteness of the algebras for a monad.

Finally, our account covers only what can be said for every well-behaved $\mathcal{V}$ (except for those things special to $\mathcal{V}=$ Set). Results valid only for a special class of $\mathcal{V}$ are best treated in separate articles, of which the author's forthcoming [45] is one example.

(iv) Our concern being to provide a development of basic category theory that covers the enriched case, we have given illustrations of many of the results in isolation, but have not thought this the place to discuss detailed applications to particular areas of mathematics. In fact, applications needing the enriched case are rapidly proliferating; the following is a selection of recent ones that have come to the author's notice.

The discussion of dualities in topological algebra, taking for $\mathcal{V}$ a suitable category of topological or quasitopological spaces, and initiated by Dubuc and Porta [23], has been continued in numerous articles by Dubuc, Day, and Nel. Pelletier [67] has made use of Kan extensions where $\mathcal{V}$ is Banach spaces. Recent Soviet work in representation theory (see the articles by Kleiner and Roiter, and by Golovaschuk, Ovsienko, and Roiter, in [64]) has differential graded modules for $\mathcal{V}$. The present author, as part of a study of essentially-algebraic structures borne by categories, has extended in [45] the classical 
results on "cartesian theories" (finite-limit theories) from the case where $\mathcal{V}$ is sets to those where it is groupoids or categories.

The study of homotopy limits by Gray [32] takes him into the area of change of basecategories, involving the connexions between the closed categories of categories and of simplicial sets. The work of May on infinite loop spaces, involving from the beginning [61] the base category $\mathcal{V}$ of compactly-generated spaces, has of late led him [62] to very general considerations on categories $\mathcal{V}$ with two symmetric monoidal structures and a distributive law between them. Mitchell [63] has related monoidal structures on $[G, \mathcal{V}]$ for suitable $\mathcal{V}$ to low-dimensional cohomology groups of the group $G$. These latter applications go beyond the basic theory presented here, but of course presuppose the relevant parts of it.

Recent work of Walters ([75], [76]) does concern the basic theory, but with $\mathcal{V}$ generalized to a symmetric monoidal closed bicategory; in this context he exhibits the sheaves on a site as the symmetric Cauchy-complete $\mathcal{V}$-categories. It is plain that continued expansion of the applications is to be expected.

(v) Writing in text-book style, we have not interrupted the development to assign credit for individual results. To do so with precision and justice would in any case be a daunting task; much has been written, and many insights were arrived at independently by several authors. Such references as do occur in the text are rather intended to show where more detail may be found. What we can do, though, is to list here some of the works to which we are particularly indebted.

Perhaps the first to advocate in print the study of enriched categories was Mac Lane [59]; although published in 1965, this represents basically his Colloquium Lectures at Boulder in 1963. There were some early attempts by Linton [56] and Kelly [40] to make a start; Bénabou ([4], [5]) went further; while Eilenberg and Kelly wrote a voluminous article [26] covering only the "elementary" notions, but including change of base-category. Our present Chapter 1 draws mainly on [26] and on the author's article [42].

The principal source for Chapter 2 is Day and Kelly [20]; but see also Bunge [10]. The ideas in Chapter 3 of cotensor products, of ends, and of limits in a $\mathcal{V}$-category, go back to [42] and [20]; and the concept of their pointwise existence in a functor-category to Dubuc [22]. However, the general notion of indexed limit, which makes Chapter 3 possible in its present form, was discovered independently by Street [72], Auderset [1], and Borceux and Kelly [9]. The last two sections of Chapter 3 call on Day's articles [12] and [14].

Chapter 4, on Kan extensions, is certainly indebted to the three articles [20], [22], [9] already mentioned; in particular it was Dubuc in [22] who pointed out the importance of the "pointwise" existence of Kan extensions, which we make part of our definition. The chapter also contains many more-or-less classical results for $\mathcal{V}=\mathbf{S e t}$; all but the best known are credited in the text to their authors.

It is especially in Chapter 5, on density, that we have been heavily influenced by writers who themselves dealt only with $\mathcal{V}=$ Set; namely Gabriel and Ulmer [31] and Diers [21]. The term "density presentation" we have taken from Day [16], although modifying its meaning somewhat. Again, writers on particular aspects are given credit in the text.

Chapter 6 shows its debt to Ehresmann [24], as well as to Gabriel and Ulmer. The form of the transfinite construction used here to prove the reflectivity of the algebras, 
although it is taken from the author's article [44], ultimately depends on an idea from an unpublished manuscript of Barr (reference [3] of [44]), which makes cowellpoweredness an unnecessary hypothesis, and so enables us to include the important example of quasitopological spaces.

The author's original contributions to the book are perhaps most visible in the arrangement of topics and in the construction of proofs that apply equally to the classical and enriched cases. For instance, many readers will find the way of introducing Kan extensions quite novel. Beyond this, the work in 5.11-5.13 on Kan extensions along a non-fully-faithful dense functor seems to be quite new even when $\mathcal{V}=$ Set, as is its application in 6.4; while the whole of Chapter 6 is new in the enriched setting.

(vi) In the early chapters the formal definition-theorem-proof style seemed out of place, and apt to lengthen an essentially simple account. Deciding to avoid it, we have accordingly made reference to a result either by quoting the section in which it occurs, or (more commonly) by quoting the number of the displayed formula most closely associated with it. In the later chapters, now more concerned with applying the logic than developing it, it seemed best to return to formally-numbered propositions and theorems.

The end-result is as follows. A symbol 5.6 without parentheses refers to section 6 of Chapter 5. All other references occur in a single series, consisting of displayed formulae and proposition- or theorem-numbers, with the first digit denoting the chapter. Thus in Chapter 5, formula (5.51) is followed by Theorem 5.52, itself followed by formula (5.53), which in fact occurs in the statement of the theorem. To assist cross-referencing, each left-hand page bears at its head the number of the last section to which that page belongs, and each right-hand page bears the numbers of the formulae, propositions, and theorems that occur on the two-page spread (in parentheses, even if they are theorem-numbers). ${ }^{1}$

(vii) It remains to thank those who have made the book possible. Over the last twelve months I have had the opportunity to present early drafts of the material to acute and stimulating audiences at the Universities of Sydney, Trieste, and Hagen; their encouragement has been of inestimable value. Equally important has been the support of my colleagues in the Sydney Category Theory Seminar - including the visiting member André Joyal, whose presence was made possible by the Australian Research Grants Committee. I am indebted to Cambridge University Press, for suggesting this Lecture Notes Series as suited to such a book, and for their careful help with the technical details of publication. It was they who observed that the original typed manuscript was already suitable as camera-ready copy; I regard this as a compliment to the intelligence and taste of my secretary Helen Rubin, whose first mathematical job this was, and I thank her very sincerely. Finally I thank my wife and children, who have borne months of shameful neglect.

\section{Max Kelly, Sydney, January 1981.}

\footnotetext{
${ }^{1}$ This has not been done in this electronic version. Instead, we use a running section title on recto pages and a running chapter title on the verso.
} 


\section{Chapter 1}

\section{The elementary notions}

\subsection{Monoidal categories}

A monoidal category $\mathcal{V}=\left(\mathcal{V}_{0}, \otimes, I, a, l, r\right)$ consists of a category $\mathcal{V}_{0}$, a functor $\otimes: \mathcal{V}_{0} \times$ $\mathcal{V}_{0} \longrightarrow \mathcal{V}_{0}$, an object $I$ of $\mathcal{V}_{0}$ and natural isomorphisms $a_{X Y Z}:(X \otimes Y) \otimes Z \longrightarrow X \otimes(Y \otimes Z)$, $l_{X}: I \otimes X \longrightarrow X, r_{X}: X \otimes I \longrightarrow X$, subject to two coherence axioms expressing the commutativity of the following diagrams:
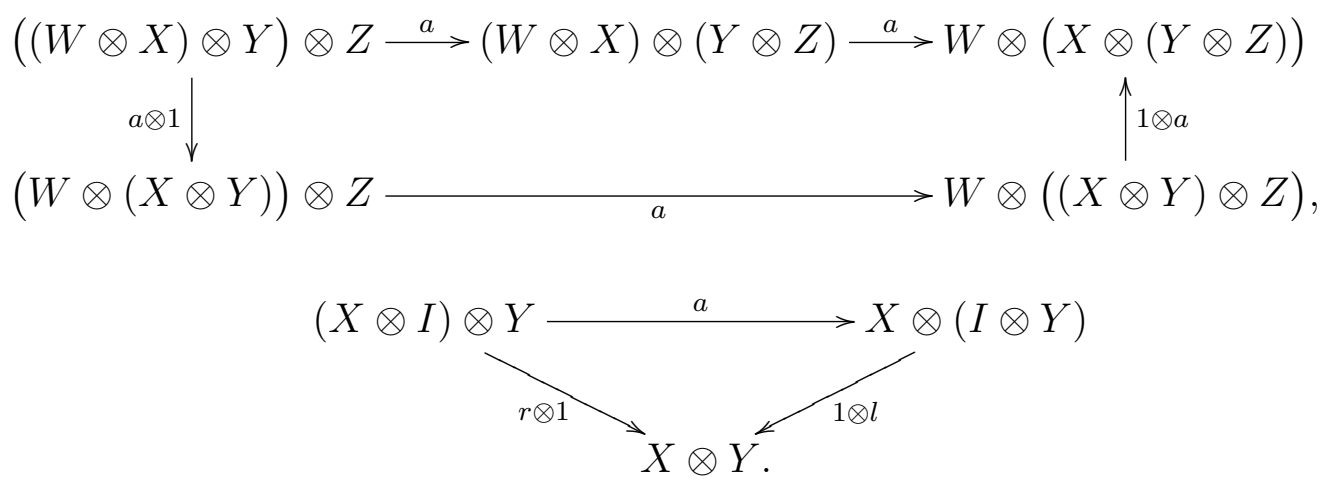

It then follows (see [58] and [39]) that every diagram of natural transformations commutes, each arrow of which is obtained by repeatedly applying the functor $\otimes$ to instances of $a$, $l, r$, their inverses, and 1 ; here an "instance" of $a$ is a natural transformation, such as $a_{W \otimes X, Y, Z}$ or $a_{X, I, Y}$ in the diagrams above, formed from $a$ by repeated application of the functors $\otimes$ and $I$ to its variables. For a precise formulation, see [58].

A special kind of example, called a cartesian monoidal category, is given by taking for $\mathcal{V}_{0}$ any category with finite products, taking for $\otimes$ and $I$ the product $\times$ and the terminal object 1 , and taking for $a, l, r$ the canonical isomorphisms. Important particular cases of this are the categories Set, Cat, Gpd, Ord, Top, CGTop, HCGTop, QTop, Shv S, of (small) sets, categories, groupoids, ordered sets, topological spaces, compactly generated topological spaces, Hausdorff such, quasitopological spaces [69], and sheaves for a site $S$; here "small" is in reference to some chosen universe, which we suppose given once and for all. Other cartesian examples are obtained by taking for $\mathcal{V}_{0}$ an ordered set with finite intersections, such as the ordinal $\mathbf{2}=\{0,1\}$. All of these examples are symmetric in the sense of 1.4 below, and all the named ones except Top are closed in the sense of 1.5 below. 
A collection of non-cartesian (symmetric, closed) examples is given by the categories Ab, $R$-Mod, G- $R$-Mod, DG- $R$-Mod of (small) abelian groups, $R$-modules for a commutative ring $R$, graded $R$-modules, and differential graded $R$-modules, each with its usual tensor product as $\otimes$; the category Ban of Banach spaces and linear maps of norm $\leqslant 1$, with the projective tensor product; the category $\mathbf{C G T o p}_{*}$ of pointed compactly-generated spaces with the smash product for $\otimes$; and the ordered set $\overline{\mathbb{R}}_{+}$of extended non-negative reals, with the reverse of the usual order, and with + as $\otimes$.

A non-symmetric example is the category of bimodules over a non-commutative ring $R$, with $\otimes_{R}$ as $\otimes$. Another is the category of endofunctors of a small category, with composition as $\otimes$; here $a, l, r$ are identities, so that the monoidal category is called strict.

In general we call $\otimes$ the tensor product, and $I$ the unit object.

We suppose henceforth given a particular monoidal $\mathcal{V}$ such that $\mathcal{V}_{0}$ is locally small (has small hom-sets). We then have the representable functor $\mathcal{V}_{0}(I,-): \mathcal{V}_{0} \longrightarrow$ Set, which we denote by $V$. In such cases as Set, Ord, Top, Ab, $R$-Mod, CGTop , $_{*}$, it is (to within isomorphism) the ordinary "underlying-set" functor; in the case of Ban, $V X$ is the unit ball of $X$; in these cases $V$ is faithful, while in some of them (Set, Ab, $R$-Mod, Ban) it is even conservative (= isomorphism-reflecting). Yet $V$ is not faithful in general; in the cases Cat and Gpd, $V X$ is the set of objects of $X$, and in the case DG- $R$-Mod, $V X$ is the set of 0-cycles.

In spite of the failure of $V$ to be faithful in general, it is convenient to call an element $f$ of $V X$ (that is, a map $f: I \longrightarrow X$ in $\mathcal{V}_{0}$ ) an element $f$ of $X$.

\subsection{The 2-category $\mathcal{V}$-CAT for a monoidal $\mathcal{V}$}

A $\mathcal{V}$-category $\mathcal{A}$ consists of a set ob $\mathcal{A}$ of objects, a hom-object $\mathcal{A}(A, B) \in \mathcal{V}_{0}$ for each pair of objects of $\mathcal{A}$, a composition law $M=M_{A B C}: \mathcal{A}(B, C) \otimes \mathcal{A}(A, B) \longrightarrow \mathcal{A}(A, C)$ for each triple of objects, and an identity element $j_{A}: I \longrightarrow \mathcal{A}(A, A)$ for each object; subject to the associativity and unit axioms expressed by the commutativity of

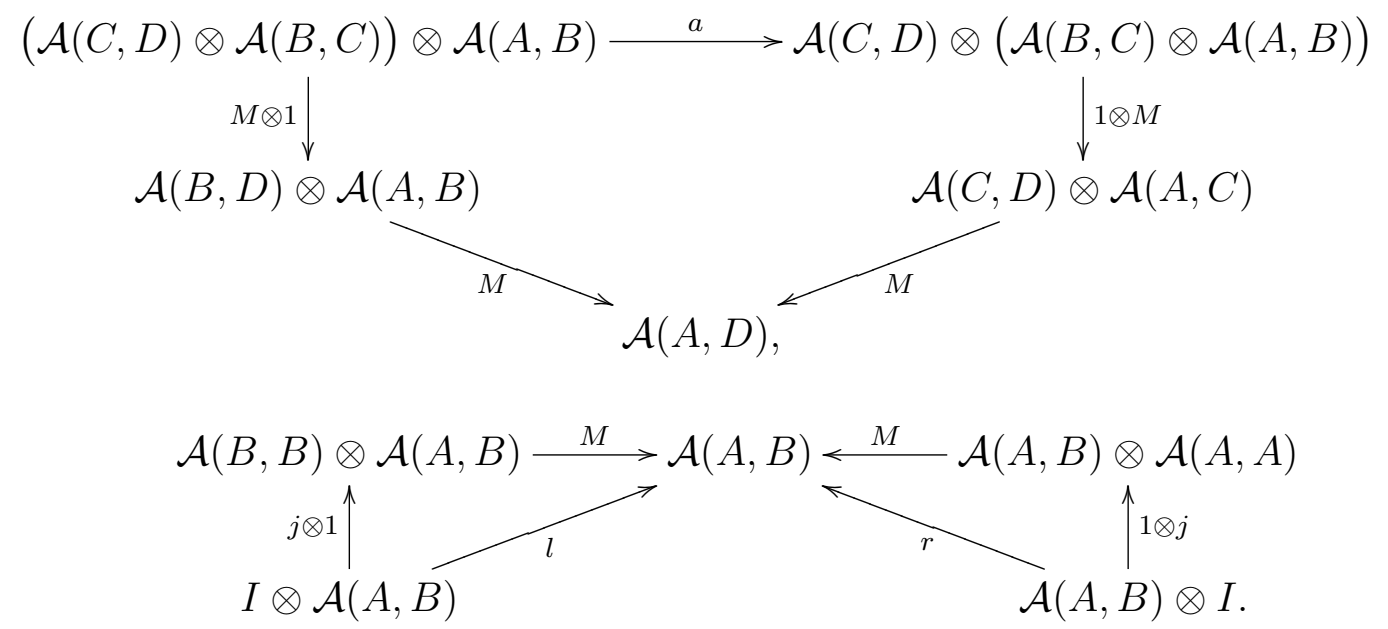

Taking $\mathcal{V}=$ Set, Cat, 2, Ab, DG- $R$-Mod, $\overline{\mathbb{R}}_{+}$, we re-find the classical notions of (locally small) ordinary category, 2-category, pre-ordered set, additive category (some 
call it "pre-additive"), differential graded category, and (rather generalized) metric space. For a general reference on 2-categories, see [49]; and for the generalized metric spaces, see [54]. We call the $\mathcal{V}$-category $\mathcal{A}$ small if ob $\mathcal{A}$ is small.

For $\mathcal{V}$-categories $\mathcal{A}$ and $\mathcal{B}$, a $\mathcal{V}$-functor $T: \mathcal{A} \longrightarrow \mathcal{B}$ consists of a function

$$
T: \operatorname{ob} \mathcal{A} \longrightarrow \text { ob } \mathcal{B}
$$

together with, for each pair $A, B \in$ ob $\mathcal{A}$, a map $T_{A B}: \mathcal{A}(A, B) \longrightarrow \mathcal{B}(T A, T B)$, subject to the compatibility with composition and with the identities expressed by the commutativity of
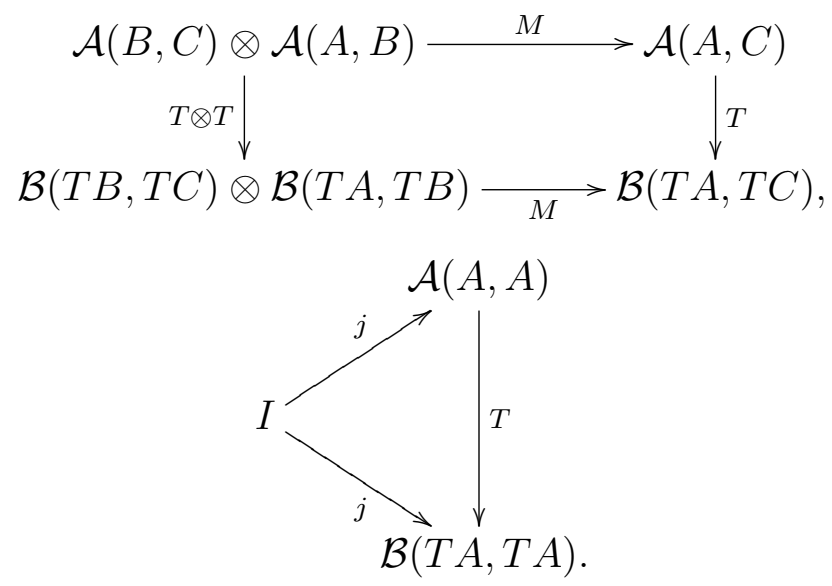

In the six examples above we re-find the classical notions of functor, 2-functor, increasing function, additive functor, differential graded functor, and contracting map. The $\mathcal{V}$ functor $T$ is fully faithful if each $T_{A B}$ is an isomorphism; an example is the inclusion $T: \mathcal{A} \longrightarrow \mathcal{B}$ of a full subcategory, determined by a subset ob $\mathcal{A}$ of ob $\mathcal{B}$. Clearly $\mathcal{V}$-functors can be composed, to form a category.

For $\mathcal{V}$-functors $T, S: \mathcal{A} \longrightarrow \mathcal{B}$, a $\mathcal{V}$-natural transformation $\alpha: T \longrightarrow S: \mathcal{A} \longrightarrow \mathcal{B}$ is an ob $\mathcal{A}$-indexed family of components $\alpha_{A}: I \longrightarrow \mathcal{B}(T A, S A)$ satisfying the $\mathcal{V}$-naturality condition expressed by the commutativity of

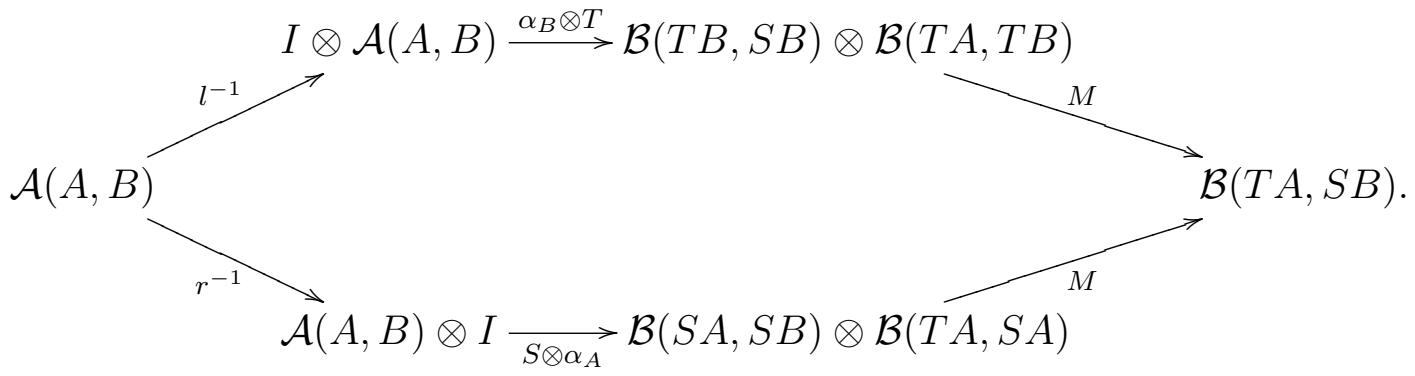

The "vertical composite" $\beta \cdot \alpha$ of $\alpha: T \longrightarrow S: \mathcal{A} \longrightarrow \mathcal{B}$ and $\beta: S \longrightarrow R: \mathcal{A} \longrightarrow \mathcal{B}$ has the component $(\beta \cdot \alpha)_{A}$ given by

$$
I \cong I \otimes I \underset{\beta_{A} \otimes \alpha_{A}}{\longrightarrow} \mathcal{B}(S A, R A) \otimes \mathcal{B}(T A, S A) \underset{M}{\longrightarrow} \mathcal{B}(T A, R A) .
$$

The composite of $\alpha$ above with $Q: \mathcal{B} \longrightarrow \mathcal{C}$ has for its component $(Q \alpha)_{A}$ the composite

$$
I \underset{\alpha_{A}}{\longrightarrow} \mathcal{B}(T A, S A) \underset{Q}{\longrightarrow} \mathcal{C}(Q T A, Q S A)
$$


while the composite of $\alpha$ with $P: \mathcal{D} \longrightarrow \mathcal{A}$ has for its component $(\alpha P)_{D}$ simply $\alpha_{P D}$.

It is now easy to verify that $\mathcal{V}$-categories, $\mathcal{V}$-functors, and $\mathcal{V}$-natural transformations constitute a 2-category $\mathcal{V}$-CAT; an "illegitimate" one, of course, unless some restriction is placed on the size, as in the legitimate 2-category $\mathcal{V}$-Cat, of small $\mathcal{V}$-categories. Of course $\mathcal{V}$-CAT reduces, when $\mathcal{V}=$ Set, to the 2-category CAT of locally small ordinary categories.

\subsection{The 2-functor ()$_{0}: \mathcal{V}$-CAT $\rightarrow$ CAT}

Denoting by $\mathcal{I}$ the unit $\mathcal{V}$-category with one object 0 and with $\mathcal{I}(0,0)=I$, we write $(-)_{0}: \mathcal{V}$-CAT $\longrightarrow$ CAT for the representable 2 -functor $\mathcal{V}-\operatorname{CAT}(\mathcal{I},-)$; which we now proceed to describe in more elementary terms.

A $\mathcal{V}$-functor $A: \mathcal{I} \longrightarrow \mathcal{A}$ may be identified with an object $A$ of the $\mathcal{V}$-category $\mathcal{A}$; and a $\mathcal{V}$-natural $f: A \longrightarrow B: \mathcal{I} \longrightarrow \mathcal{A}$ consists of a single component $f: I \longrightarrow \mathcal{A}(A, B)$, the axiom (1.7) being trivially satisfied. Thus the ordinary category $\mathcal{A}_{0}=\mathcal{V}$ - $\operatorname{CAT}(\mathcal{I}, \mathcal{A})$, which is called the underlying category of $\mathcal{A}$, has the same objects as $\mathcal{A}$, while a map $f: A \longrightarrow B$ in $\mathcal{A}_{0}$ is just an element $f: I \longrightarrow \mathcal{A}(A, B)$ of $\mathcal{A}(A, B)$, in the sense of 1.1. Otherwise put, $\mathcal{A}_{0}(A, B)=V \mathcal{A}(A, B)$. By $(1.8)$, the composite $g f$ in $\mathcal{A}_{0}$ is given by the composite

$$
I \cong I \otimes I \underset{g \otimes f}{\longrightarrow} \mathcal{A}(B, C) \otimes \mathcal{A}(A, B) \underset{M}{\longrightarrow} \mathcal{A}(A, C)
$$

in $\mathcal{V}_{0}$, while the identity in $\mathcal{A}_{0}(A, A)$ is clearly $j_{A}$.

How much information about $\mathcal{A}$ is retained by $\mathcal{A}_{0}$ depends upon how faithful $V$ is. When $\mathcal{V}=$ Cat, $V$ is not faithful; $\mathcal{A}$ is a 2-category, and $\mathcal{A}_{0}$ is the category obtained by discarding the 2-cells. When $\mathcal{V}=$ CGTop, $V$ is faithful, and $\mathcal{A}_{0}$ has lost only the topology on the hom-objects of $\mathcal{A}$. When $\mathcal{V}=\mathrm{Ab}$ or $R$-Mod, $V$ is even conservative, and $\mathcal{A}_{0}$ is still closer to $\mathcal{A}$.

The ordinary functor $T_{0}: \mathcal{A}_{0} \longrightarrow \mathcal{B}_{0}$ induced by (or underlying) the $\mathcal{V}$-functor $T: \mathcal{A} \longrightarrow \mathcal{B}$ sends $A: \mathcal{I} \longrightarrow \mathcal{A}$ to $T A$ and sends $f: A \longrightarrow B: \mathcal{I} \longrightarrow \mathcal{A}$ to $T f$, which by (1.9) is the composite

$$
I \underset{f}{\longrightarrow} \mathcal{A}(A, B) \underset{T_{A B}}{\longrightarrow} \mathcal{B}(T A, T B) .
$$

Thus we have

$$
T_{0} A=T A, \quad T_{0} f=T f ;
$$

the latter of which means that

$$
T_{0 A B}: \mathcal{A}_{0}(A, B) \longrightarrow \mathcal{B}_{0}(T A, T B) \text { is } \quad V T_{A B}: V \mathcal{A}(A, B) \longrightarrow V \mathcal{B}(T A, T B)
$$

Clearly $T_{0}$ is fully faithful if $T$ is; $T \mapsto T_{0}$ is injective when $V$ is faithful, but not in general; and, when $V$ is conservative, $T$ is fully faithful if $T_{0}$ is.

The ordinary natural transformation $\alpha_{0}: T_{0} \longrightarrow S_{0}: \mathcal{A}_{0} \longrightarrow \mathcal{B}_{0}$ induced by the $\mathcal{V}$-natural $\alpha: T \longrightarrow S: \mathcal{A} \longrightarrow \mathcal{B}$ has for its $A$-component $\alpha_{0 A} \in \mathcal{B}_{0}(T A, S A)$ precisely the $A$-component $\alpha_{A}: I \longrightarrow \mathcal{B}(T A, S A)$ of $\alpha$; so that it is not usually necessary to distinguish $\alpha$ from $\alpha_{0}$. The $\mathcal{V}$-naturality condition (1.7) for $\alpha: T \longrightarrow S$ becomes the usual naturality condition for 
$\alpha: T_{0} \longrightarrow S_{0}$ when $V$ is applied to it. Hence the naturality of $\alpha: T_{0} \longrightarrow S_{0}$, while weaker in general than the $\mathcal{V}$-naturality of $\alpha: T \longrightarrow S$, is equivalent to it when $V$ is faithful.

In spite of such formulae as (1.12), both clarity and economy are served by carefully distinguishing $\mathcal{A}$ from $\mathcal{A}_{0}$ and $T$ from $T_{0}$. For instance, completeness of $\mathcal{A}$, to be defined below, means something stronger than completeness of $\mathcal{A}_{0}$; and continuity of $T$ means something stronger than continuity of $T_{0}$. Having a left adjoint for $T$ is stronger than having a left adjoint for $T_{0}$; while the existence of a small dense subcategory of $\mathcal{A}$ neither implies nor is implied by the existence of a small dense subcategory of $\mathcal{A}_{0}$. As for economy, maintaining the distinction allows us, when $\mathcal{A}$ and $\mathcal{B}$ are $\mathcal{V}$-categories, to abbreviate "V-functor $T: \mathcal{A} \longrightarrow \mathcal{B}$ " to "functor (or map) $T: \mathcal{A} \longrightarrow \mathcal{B}$ "; if we had meant a functor $T: \mathcal{A}_{0} \longrightarrow \mathcal{B}_{0}$, we should have said so. Again, to speak of "a $\mathcal{V}$-functor $T: \mathcal{A} \longrightarrow \mathcal{B}$ " carries the converse implication, that $\mathcal{A}$ and $\mathcal{B}$ are $\mathcal{V}$-categories. Similarly, when $T, S: \mathcal{A} \longrightarrow \mathcal{B}$ are $\mathcal{V}$-functors, by "map (or natural transformation) $\alpha: T \longrightarrow S$ " we must mean a $\mathcal{V}$-natural one; for we did not speak of a map $\alpha: T_{0} \longrightarrow S_{0}$. When only components are written, however, it may be necessary to say " $\alpha_{A}: T A \longrightarrow S A$ is $\mathcal{V}$-natural in $A$ ", since $T A$ is also $T_{0} A$. Finally, since strictly speaking there are no "morphisms" in the $\mathcal{V}$-category $\mathcal{A}$, it is harmless to call a map $f: A \longrightarrow B$ in $\mathcal{A}_{0}$, which is an element $f: I \longrightarrow \mathcal{A}(A, B)$ of $\mathcal{A}(A, B)$, "a map $f: A \longrightarrow B$ in $\mathcal{A}$ ".

\subsection{Symmetric monoidal categories: the tensor product and duality on $\mathcal{V}$-CAT for a symmetric monoidal $\mathcal{V}$}

A symmetry $c$ for a monoidal category $\mathcal{V}$ is a natural isomorphism $c_{X Y}: X \otimes Y \rightarrow Y \otimes X$ satisfying the coherence axioms expressed by the commutativity of

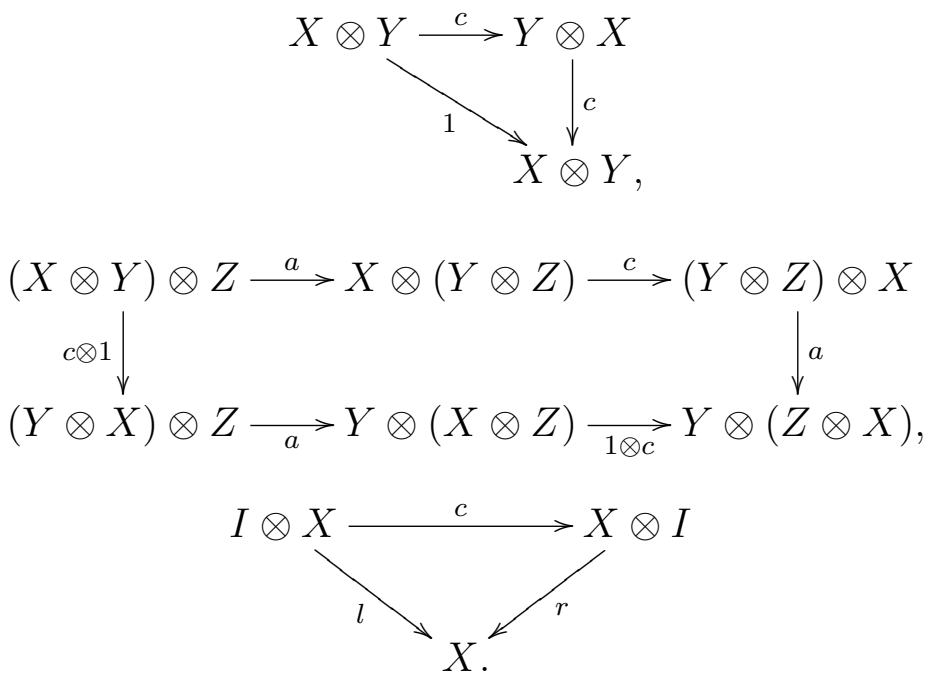

Note that (1.16) defines $l$ in terms of $r$, and then we need only the four coherence axioms (1.1), (1.2), (1.14), (1.15). It follows from [58] and [39] that every diagram of natural transformations commutes, each arrow of which is obtained by the repeated application of $\otimes$ to instances of $a, l, r, c$, their inverses, and 1 ; cf. 1.1 , and for a precise formulation, see again [58]. 
A monoidal category - even a closed one in the sense of 1.5 below - may admit more than one symmetry. The classical example is $\mathcal{V}=\mathbf{G}-R$-Mod, with $c(x \otimes y)$, for homogeneous elements $x, y$ of degrees $p, q$, defined either as $y \otimes x$ or as $(-1)^{p q} y \otimes x$. However, if $V$ is faithful and $\mathcal{V}$ is closed, there is at most one symmetry ([26] Chapter III, Proposition 6.1). For a cartesian $\mathcal{V}$, there is an evident canonical $c: X \otimes Y \longrightarrow Y \otimes X$. For the cases Ab, $R$-Mod, Ban, and $\mathbf{C G T o p}_{*}, V$ is faithful and the unique symmetry is well-known. For DG- $R$-Mod the classical symmetry is given by $c(x \otimes y)=(-1)^{p q} y \otimes x$; and for $\overline{\mathbb{R}}_{+}$it is the equality $x+y=y+x$.

A monoidal category $\mathcal{V}$ together with a symmetry is called a symmetric monoidal category. We now suppose $\mathcal{V}$ to be such.

Then to each pair $\mathcal{A}, \mathcal{B}$ of $\mathcal{V}$-categories we can associate a tensor product $\mathcal{A} \otimes \mathcal{B}$, with object-set ob $\mathcal{A} \times$ ob $\mathcal{B}$, and with $(A \otimes B)\left((A, B),\left(A^{\prime}, B^{\prime}\right)\right)=\mathcal{A}\left(A, A^{\prime}\right) \otimes \mathcal{B}\left(B, B^{\prime}\right)$. The composition-law is given by the top edge of

$$
\begin{aligned}
& \left(\mathcal{A}\left(A^{\prime}, A^{\prime \prime}\right) \otimes \mathcal{B}\left(B^{\prime}, B^{\prime \prime}\right)\right) \otimes\left(\mathcal{A}\left(A, A^{\prime}\right) \otimes \mathcal{B}\left(B, B^{\prime}\right)\right) \stackrel{M}{\longrightarrow} \mathcal{A}\left(A, A^{\prime \prime}\right) \otimes \mathcal{B}\left(B, B^{\prime \prime}\right) \\
& m \downarrow \\
& \left(\mathcal{A}\left(A^{\prime}, A^{\prime \prime}\right) \otimes \mathcal{A}\left(A, A^{\prime}\right)\right) \otimes\left(\mathcal{B}\left(B^{\prime}, \widehat{B^{\prime \prime}}\right) \otimes \mathcal{B}\left(B, B^{\prime}\right)\right),
\end{aligned}
$$

where $m:(W \otimes X) \otimes(Y \otimes Z) \cong(W \otimes Y) \otimes(X \otimes Z)$ is the middle-four interchange defined by any suitable composite of instances of $a$ and of $c$. The identity element is the composite

$$
I \cong I \otimes I \underset{j_{A} \otimes j_{B}}{\longrightarrow} \mathcal{A}(A, A) \otimes \mathcal{B}(B, B)
$$

and axioms (1.3) and (1.4) are easy to verify.

It is further easy to check that, with the obvious definitions of $T \otimes S: \mathcal{A} \otimes \mathcal{B} \longrightarrow \mathcal{A}^{\prime} \otimes \mathcal{B}^{\prime}$ and of $\alpha \otimes \beta: T \otimes S \longrightarrow T^{\prime} \otimes S^{\prime}$, we have a 2-functor $\otimes: \mathcal{V}$-CAT $\times \mathcal{V}$-CAT $\longrightarrow \mathcal{V}$-CAT; and that we have coherent 2-natural isomorphisms $(\mathcal{A} \otimes B) \otimes C \cong \mathcal{A} \otimes(\mathcal{B} \otimes \mathcal{C}), \mathcal{I} \otimes \mathcal{A} \cong$ $\mathcal{A} \otimes \mathcal{I} \cong \mathcal{A}$, and $\mathcal{A} \otimes \mathcal{B} \cong \mathcal{B} \otimes \mathcal{A}$ (where $\mathcal{I}$ is the unit $\mathcal{V}$-category of 1.3 ). Thus $\mathcal{V}$-CAT is, in an evident sense, a symmetric monoidal 2-category.

Moreover, to each $\mathcal{V}$-category $\mathcal{A}$ we can associate a dual, or opposite, $\mathcal{V}$-category $\mathcal{A}^{\text {op }}$, with the same objects as $\mathcal{A}$, but with $\mathcal{A}^{\mathrm{op}}(A, B)=\mathcal{A}(B, A)$. The composition-law $M: \mathcal{A}^{\mathrm{op}}(B, C) \otimes \mathcal{A}^{\mathrm{op}}(A, B) \longrightarrow \mathcal{A}^{\mathrm{op}}(A, C)$ is just the composite

$$
\mathcal{A}(C, B) \otimes \mathcal{A}(B, A) \underset{c}{\longrightarrow} \mathcal{A}(B, A) \otimes \mathcal{A}(C, B) \underset{M}{\longrightarrow} \mathcal{A}(C, A)
$$

while the unit element $I \longrightarrow \mathcal{A}^{\mathrm{op}}(A, A)$ is that of $\mathcal{A}$. From $\alpha: T \longrightarrow S: \mathcal{A} \longrightarrow \mathcal{B}$ we get, with evident definitions, $\alpha^{\mathrm{op}}: S^{\mathrm{op}} \longrightarrow T^{\mathrm{op}}: \mathcal{A}^{\mathrm{op}} \longrightarrow \mathcal{B}^{\mathrm{op}}$; note that $(-)^{\text {op }}$ reverses 2 -cells but not 1-cells. Of course $(-)^{\mathrm{op}}$ is involutary: $\left(\mathcal{A}^{\mathrm{op}}\right)^{\mathrm{op}}=\mathcal{A}$; and it respects tensor products: $(\mathcal{A} \otimes \mathcal{B})^{\text {op }}=\mathcal{A}^{\text {op }} \otimes \mathcal{B}^{\text {op }}$. Thus, in an evident sense, $\mathcal{V}$-CAT is a symmetric monoidal 2-category with a duality involution.

A functor $\mathcal{A}^{\mathrm{op}} \longrightarrow \mathcal{B}$ may be called a contravariant functor from $\mathcal{A}$ to $\mathcal{B}$; while a functor $T: \mathcal{A} \otimes \mathcal{B} \longrightarrow \mathcal{C}$ may be thought of as a functor of two variables. Such a $T$ gives rise to partial functors $T(A,-): \mathcal{B} \longrightarrow \mathcal{C}$ for each $A \in \mathcal{A}$ and $T(-, B): \mathcal{A} \longrightarrow \mathcal{C}$ for each $B \in \mathcal{B}$. 
Here $T(A,-)$ is the composite

$$
\mathcal{B} \cong \mathcal{I} \otimes \mathcal{B} \underset{A \otimes 1}{\longrightarrow} \mathcal{A} \otimes \mathcal{B} \underset{T}{\longrightarrow} \mathcal{C}
$$

from which can be read off the value of $T(A,-)_{B B^{\prime}}$.

Suppose, conversely, that we are given a family of functors $T(A,-): \mathcal{B} \longrightarrow \mathcal{C}$ indexed by ob $\mathcal{A}$ and a family of functors $T(-, B): \mathcal{A} \longrightarrow \mathcal{C}$ indexed by ob $\mathcal{B}$, such that on objects we have $T(A,-) B=T(-, B) A,=T(A, B)$, say. Then there is a functor $T: \mathcal{A} \otimes \mathcal{B} \longrightarrow \mathcal{C}$ of which these are the partial functors if and only if we have commutativity in

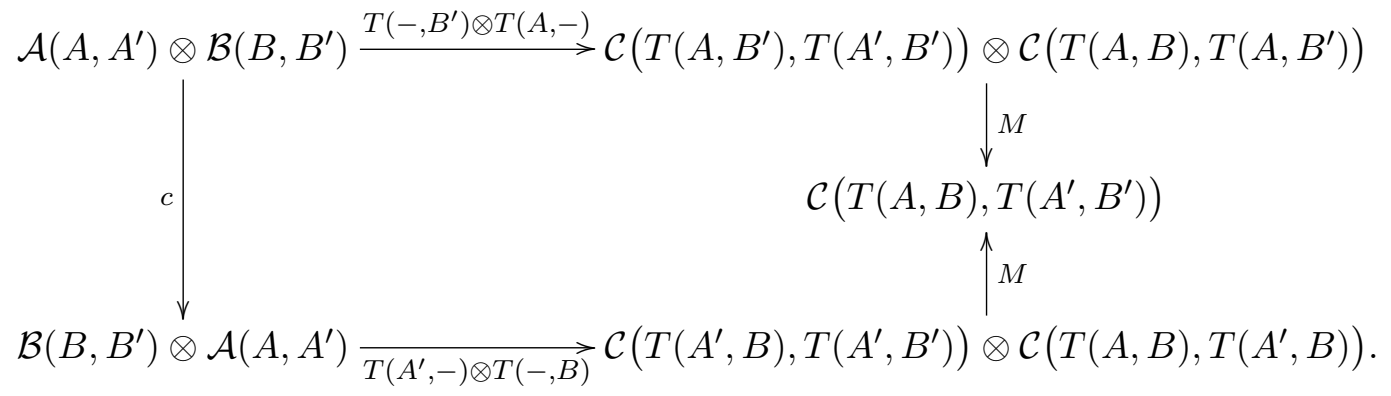

Moreover $T$ is then unique, and $T_{(A, B),\left(A^{\prime}, B^{\prime}\right)}$ is the diagonal of (1.21). The verification is easy, and the details can be found in ([26] Chapter III, §4).

It is further easy (using (1.21), and (1.3) for $\mathcal{C}$ ) to verify that, if $T, S: \mathcal{A} \otimes \mathcal{B} \longrightarrow \mathcal{C}$, a family $\alpha_{A B}: T(A, B) \longrightarrow S(A, B)$ in $\mathcal{C}_{0}$ constitutes a $\mathcal{V}$-natural transformation $T \longrightarrow S$ if and only if it constitutes, for each fixed $A$, a $\mathcal{V}$-natural $T(A,-) \longrightarrow S(A,-)$, and, for each fixed $B$, a $\mathcal{V}$-natural $T(-, B) \longrightarrow S(-, B)$. In other words, $\mathcal{V}$-naturality may be verified variable-by-variable.

In relation to the underlying ordinary categories, we have of course $\left(\mathcal{A}^{\mathrm{op}}\right)_{0}=\left(\mathcal{A}_{0}\right)^{\mathrm{op}}$ and $\left(T^{\mathrm{op}}\right)_{0}=\left(T_{0}\right)^{\mathrm{op}}$. However, $(\mathcal{A} \otimes \mathcal{B})_{0}$ is not $\mathcal{A}_{0} \times \mathcal{B}_{0}$; rather there is an evident canonical functor $\mathcal{A}_{0} \times \mathcal{B}_{0} \longrightarrow(\mathcal{A} \otimes \mathcal{B})_{0}$, and a similar one $1 \longrightarrow \mathcal{I}_{0}$. For $T: \mathcal{A} \otimes \mathcal{B} \longrightarrow \mathcal{C}$, the partial functors of the composite ordinary functor

$$
\mathcal{A}_{0} \times \mathcal{B}_{0} \longrightarrow(\mathcal{A} \otimes \mathcal{B})_{0} \underset{T_{0}}{\longrightarrow} \mathcal{C}_{0}
$$

are precisely $T(A,-)_{0}$ and $T(-, B)_{0}$.

We could discuss, in the present setting, the extraordinary $\mathcal{V}$-natural families of 1.7 below. However, the techniques that make this simple arise more naturally, and at a higher level, when $\mathcal{V}$ is closed; which is the case of real interest. Moreover it is shown in [17] that any symmetric monoidal category can, by passing to a higher universe, be embedded in a closed one; see also 2.6 and 3.11 below.

\subsection{Closed and biclosed monoidal categories}

The monoidal category $\mathcal{V}$ (symmetric or not) is said to be closed (cartesian closed, when $\mathcal{V}$ is cartesian monoidal) if each functor $-\otimes Y: \mathcal{V}_{0} \longrightarrow \mathcal{V}_{0}$ has a right adjoint $[Y,-]$; so that we have an adjunction

$$
\pi: \mathcal{V}_{0}(X \otimes Y, Z) \cong \mathcal{V}_{0}(X,[Y, Z])
$$


with unit and counit (the latter called evaluation) say

$$
d: X \longrightarrow[Y, X \otimes Y], \quad e:[Y, Z] \otimes Y \longrightarrow Z .
$$

Putting $X=I$ in (1.23), using the isomorphism $l: I \otimes Y \cong Y$, and recalling from 1.1 that $V=\mathcal{V}_{0}(I,-): \mathcal{V}_{0} \longrightarrow$ Set, we get a natural isomorphism

$$
\mathcal{V}_{0}(Y, Z) \cong V[Y, Z]
$$

since $[Y, Z]$ is thus exhibited as a lifting through $V$ of the hom-set $\mathcal{V}_{0}(Y, Z)$, it is called the internal hom of $Y$ and $Z$.

Putting $Y=I$ in (1.23) and using the isomorphism $r: X \otimes I \cong X$, we deduce a natural isomorphism

$$
i: Z \cong[I, Z]
$$

it is easy to verify that $V i: V Z \cong V[I, Z]$ is just the case $Y=I$ of (1.25). Replacing $X$ by $W \otimes X$ in (1.23), and using the isomorphism $a:(W \otimes X) \otimes Y \cong W \otimes(X \otimes Y)$, we deduce a natural isomorphism

$$
p:[X \otimes Y, Z] \cong[X,[Y, Z]]
$$

and it easy to verify that $V p$ agrees with the $\pi$ of (1.23) modulo the isomorphisms (1.25). In many concrete cases it is possible and convenient to replace $V$ with an isomorph in such a way that (1.25) becomes an equality; this should be clear from the examples of closed categories given in 1.1, and we do not labour the point.

The monoidal category $\mathcal{V}$ is said to be biclosed if, not only does every $-\otimes Y$ have a right adjoint $[Y,-]$, but also every $X \otimes-$ has a right adjoint $\llbracket X,-\rrbracket$. When $\mathcal{V}$ is symmetric, it is biclosed if closed, with $\llbracket X,-\rrbracket=[X,-]$. The non-symmetric monoidal category of $R$-bimodules, mentioned in 1.1, is biclosed. It is part of a larger structure, the biclosed bicategory of left- $R$-, right- $S$-, bimodules, where $R$ and $S$ vary over all rings; and it seems to be typical that non-symmetric biclosed monoidal categories occur in nature as a small part of such a bicategory. (The notion of bicategory, for an exposition of which see [6], generalises that of 2-category, the strict associativity of 1-cells being replaced by associativity to within coherent isomorphisms; a one-object bicategory is a monoidal category, just as a one-object 2-category is a strict monoidal category.) Recent work of R.F.C. Walters ([75], [76]) suggests the importance of studying " $\mathcal{V}$-categories $\mathcal{A}$ " where $\mathcal{V}$ is a biclosed bicategory with an appropriate kind of symmetry; but that goes beyond the scope of the present account.

There also do occur in nature important closed monoidal categories that are not biclosed. A typical example is given by the strict monoidal category of small endofunctors (in some suitable sense of "small") of a well-behaved large category such as Set; see [45]. For such a monoidal closed $\mathcal{V}$, we can develop part of the theory of $\mathcal{V}$-categories; we have the results of 1.2 and 1.3, but not those of 1.4; and we have the Yoneda Lemma of 1.9 below, but not its extra-variable form. However, we do not pursue this at all, for in practice it seems that the interest in such a $\mathcal{V}$ lies in $\mathcal{V}$ itself, not in $\mathcal{V}$-categories - which become interesting chiefly when $\mathcal{V}$ is symmetric monoidal closed. 
We end this section with two further comments on examples. First, the symmetric (cartesian) monoidal Top is not closed: $-\times Y$ cannot have a right adjoint since it does not preserve regular epimorphisms; see [19]. Next, to the examples of symmetric monoidal closed category in 1.1 , we add one more class. An ordered set $\mathcal{V}$ with finite intersections and finite unions is called a Heyting algebra if it is cartesian closed; a boolean algebra is a special case.

\section{$1.6 \mathcal{V}$ as a $\mathcal{V}$-category for symmetric monoidal closed $\mathcal{V}$; representable $\mathcal{V}$-functors}

From now on we suppose that our given $\mathcal{V}$ is symmetric monoidal closed, with $\mathcal{V}_{0}$ locally small. The structure of $\mathcal{V}$-CAT then becomes rich enough to permit of Yoneda-lemma arguments formally identical with those in CAT. Before giving the Yoneda Lemma and its extensions in 1.9, we collect in this section and the next two some necessary preliminaries: results many of which are almost trivial in CAT, but less so here.

The proof of each assertion of these sections is the verification of the commutativity of a more-or-less large diagram. This verification can be done wholesale, in that each diagram involved is trivially checked to be of the type proved commutative in the coherence theorems of [47] and [48]. Yet direct verifications, although somewhat tedious, are nevertheless fairly straightforward if the order below is followed.

The first point is that the internal-hom of $\mathcal{V}$ "makes $\mathcal{V}$ itself into a $\mathcal{V}$-category". More precisely, there is a $\mathcal{V}$-category, which we call $\mathcal{V}$, whose objects are those of $\mathcal{V}_{0}$, and whose hom-object $\mathcal{V}(X, Y)$ is $[X, Y]$. Its composition-law $M:[Y, Z] \otimes[X, Y] \longrightarrow[X, Z]$ corresponds under the adjunction (1.23) to the composite

$$
([Y, Z] \otimes[X, Y]) \otimes X \underset{a}{\longrightarrow}[Y, Z] \otimes([X, Y] \otimes X) \underset{1 \otimes e}{\longrightarrow}[Y, Z] \otimes Y \underset{e}{\longrightarrow} Z,
$$

and its identity element $j_{X}: I \longrightarrow[X, X]$ corresponds under $(1.23)$ to $l: I \otimes X \longrightarrow X$. Verification of the axioms (1.3) and (1.4) is easy when we recall that, because of the relation of $e$ to the $\pi$ of (1.23), the definition (1.28) of $M$ is equivalent to $e(M \otimes 1)=$ $e(1 \otimes e) a$. It is further easily verified that (1.25) gives an isomorphism between $\mathcal{V}_{0}$ and the underlying ordinary category of the $\mathcal{V}$-category $\mathcal{V}$; we henceforth identify these two ordinary categories by this isomorphism, thus rendering the notation $\mathcal{V}_{0}$ consistent with the notation $\mathcal{A}_{0}$ of 1.3 .

Next we observe that, for each $\mathcal{V}$-category $\mathcal{A}$ and each object $A \in \mathcal{A}$, we have the representable $\mathcal{V}$-functor $\mathcal{A}(A,-): \mathcal{A} \longrightarrow \mathcal{V}$ sending $B \in \mathcal{A}$ to $\mathcal{A}(A, B) \in \mathcal{V}$, and with

$$
\mathcal{A}(A,-)_{B C}: \mathcal{A}(B, C) \longrightarrow[\mathcal{A}(A, B), \mathcal{A}(A, C)]
$$

corresponding under the adjunction (1.23) to $M: \mathcal{A}(B, C) \otimes \mathcal{A}(A, B) \longrightarrow \mathcal{A}(A, C)$. Axioms (1.5) and (1.6) for a $\mathcal{V}$-functor reduce easily to (1.3) and half of (1.4).

Replacing $\mathcal{A}$ by $\mathcal{A}^{\mathrm{op}}$ gives the contravariant representable functor $\mathcal{A}(-, B): \mathcal{A}^{\mathrm{op}} \longrightarrow \mathcal{V}$. The families $\mathcal{A}(A,-)$ and $\mathcal{A}(-, B)$ are the partial functors of a functor $\mathrm{Hom}_{\mathcal{A}}: \mathcal{A}^{\text {op }} \otimes$ $\mathcal{A} \longrightarrow \mathcal{V}$ sending $(A, B)$ to $\mathcal{A}(A, B)$; for the condition (1.21) again reduces easily to (1.3) for $\mathcal{A}$. 
We write $\operatorname{hom}_{\mathcal{A}}: \mathcal{A}_{0}^{\text {op }} \times \mathcal{A}_{0} \longrightarrow \mathcal{V}_{0}$ for the ordinary functor

$$
\mathcal{A}_{0}^{\mathrm{op}} \times \mathcal{A}_{0} \longrightarrow\left(\mathcal{A}^{\mathrm{op}} \otimes \mathcal{A}\right)_{0} \underset{\left(\mathrm{Hom}_{\mathcal{A}}\right)_{0}}{\longrightarrow} \mathcal{V}_{0}
$$

it sends $(A, B)$ to $\mathcal{A}(A, B)$, and for its value on maps we write

$$
\mathcal{A}(f, g): \mathcal{A}(A, B) \longrightarrow \mathcal{A}\left(A^{\prime}, B^{\prime}\right),
$$

where $f: A^{\prime} \longrightarrow A$ and $g: B \longrightarrow B^{\prime}$. By 1.4, the partial functors of hom $\mathcal{A}_{\mathcal{A}}$ are the functors underlying $\mathcal{A}(A,-)$ and $\mathcal{A}(-, B)$, so that our writing their values on morphisms as $\mathcal{A}(A, g)$ and $\mathcal{A}(f, B)$ is consistent with (1.12). Combining (1.11) with the definition (1.29) of $\mathcal{A}(A,-)_{B C}$, we see that $\mathcal{A}(A, g): \mathcal{A}(A, B) \longrightarrow \mathcal{A}(A, C)$ is the composite

$$
\mathcal{A}(A, B) \underset{l^{-1}}{\longrightarrow} I \otimes \mathcal{A}(A, B) \underset{g \otimes 1}{\longrightarrow} \mathcal{A}(B, C) \otimes \mathcal{A}(A, B) \underset{M}{\longrightarrow} \mathcal{A}(A, C),
$$

while $\mathcal{A}(f, B): \mathcal{A}(D, B) \longrightarrow \mathcal{A}(A, B)$ is

$$
\mathcal{A}(D, B) \underset{r^{-1}}{\longrightarrow} \mathcal{A}(D, B) \otimes I \underset{1 \otimes f}{\longrightarrow} \mathcal{A}(D, B) \otimes \mathcal{A}(A, D) \underset{M}{\longrightarrow} \mathcal{A}(A, B) .
$$

From these it follows easily that we have commutativity in

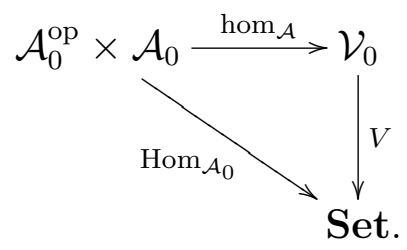

When $\mathcal{A}=\mathcal{V}$, we see at once that hom $\mathcal{V}$ is just the functor $[-,-]: \mathcal{V}_{0}^{\mathrm{op}} \times \mathcal{V}_{0} \longrightarrow \mathcal{V}_{0}$

Now we observe that there is also a $\mathcal{V}$-functor Ten: $\mathcal{V} \otimes \mathcal{V} \longrightarrow \mathcal{V}$, sending

$$
(X, Y) \longrightarrow \operatorname{Ten}(X, Y)=X \otimes Y
$$

and with $\operatorname{Ten}_{(X, Y),\left(X^{\prime}, Y^{\prime}\right)}:\left[X, X^{\prime}\right] \otimes\left[Y, Y^{\prime}\right] \longrightarrow\left[X \otimes Y, X^{\prime} \otimes Y^{\prime}\right]$ corresponding under the adjunction (1.23) to the composite

$$
\left(\left[X, X^{\prime}\right] \otimes\left[Y, Y^{\prime}\right]\right) \otimes(X \otimes Y) \underset{m}{\longrightarrow}\left(\left[X, X^{\prime}\right] \otimes X\right) \otimes\left(\left[Y, Y^{\prime}\right] \otimes Y\right) \underset{e \otimes e}{\longrightarrow} X^{\prime} \otimes Y^{\prime}
$$

when we observe that $(1.34)$ is equivalent to $e(\operatorname{Ten} \otimes 1)=(e \otimes e) m$, verification of the $\mathcal{V}$-functor axioms (1.5) and (1.6) is easy. The ordinary functor

$$
\mathcal{V}_{0} \times \mathcal{V}_{0} \longrightarrow(\mathcal{V} \otimes \mathcal{V})_{0} \underset{\operatorname{Ten}_{0}}{\longrightarrow} \mathcal{V}_{0}
$$

is at once seen to be $\otimes: \mathcal{V}_{0} \times \mathcal{V}_{0} \longrightarrow \mathcal{V}_{0}$; so that, by (1.4), the underlying ordinary functor of $\operatorname{Ten}(X,-)$ is $X \otimes-$. 
For any $\mathcal{V}$-categories $\mathcal{A}$ and $\mathcal{B}$ we have a commutative diagram

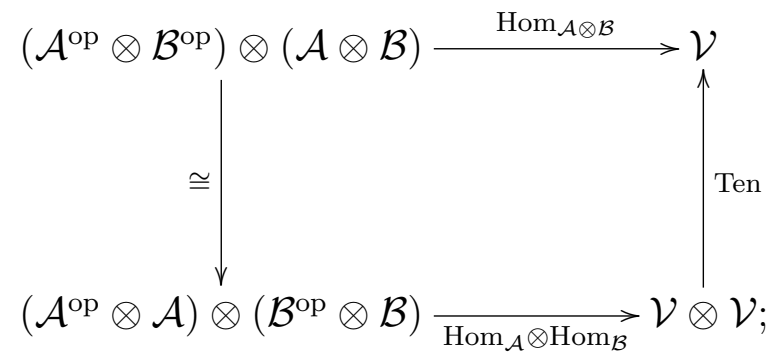

in terms of the partial functors, this asserts the commutativity of

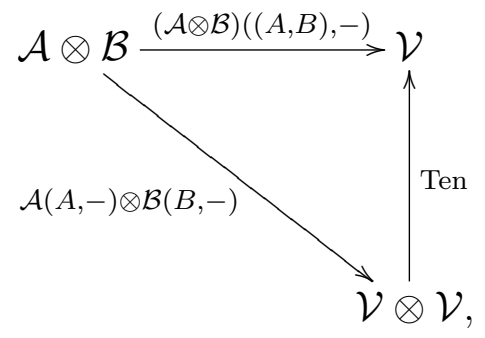

which is easily checked. At the level of the underlying functors hom, this gives

$$
(A \otimes B)\left((f, g),\left(f^{\prime}, g^{\prime}\right)\right)=\mathcal{A}\left(f, f^{\prime}\right) \otimes \mathcal{B}\left(g, g^{\prime}\right) .
$$

\subsection{Extraordinary $\mathcal{V}$-naturality}

The point to be made in the next section is that all the families of maps canonically associated to $\mathcal{V}$, or to a $\mathcal{V}$-category $\mathcal{A}$, or to a $\mathcal{V}$-functor $T$, or to a $\mathcal{V}$-natural $\alpha$, such as $a:(X \otimes Y) \otimes Z \longrightarrow X \otimes(Y \otimes Z)$, or $e:[Y, Z] \otimes Y \longrightarrow Z$, or $M: \mathcal{A}(B, C) \otimes \mathcal{A}(A, B) \longrightarrow \mathcal{A}(A, C)$, or $T: \mathcal{A}(A, B) \longrightarrow \mathcal{B}(T A, T B)$, or $\alpha: I \longrightarrow \mathcal{B}(T A, S A)$, are themselves $\mathcal{V}$-natural in every variable. To deal with a variable like $B$ in the case of $M$, we must now introduce the notion of extraordinary $\mathcal{V}$-naturality; which later plays an essential role in the definition of $\mathcal{V}$-functor-category.

First we observe that the formulae (1.31) and (1.32) allow us to write the "ordinary" $\mathcal{V}$-naturality condition (1.7) in the more compact form

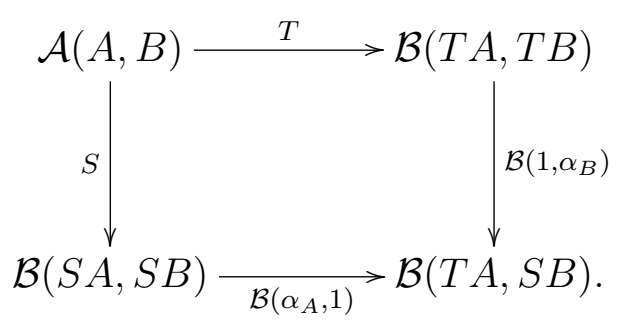

By (extraordinary) $\mathcal{V}$-naturality for an ob $\mathcal{A}$-indexed family of maps $\beta_{A}: K \longrightarrow T(A, A)$ 
in $\mathcal{B}_{0}$, where $K \in \mathcal{B}$ and $T: \mathcal{A}^{\text {op }} \otimes \mathcal{A} \longrightarrow \mathcal{B}$, we mean the commutativity of each diagram

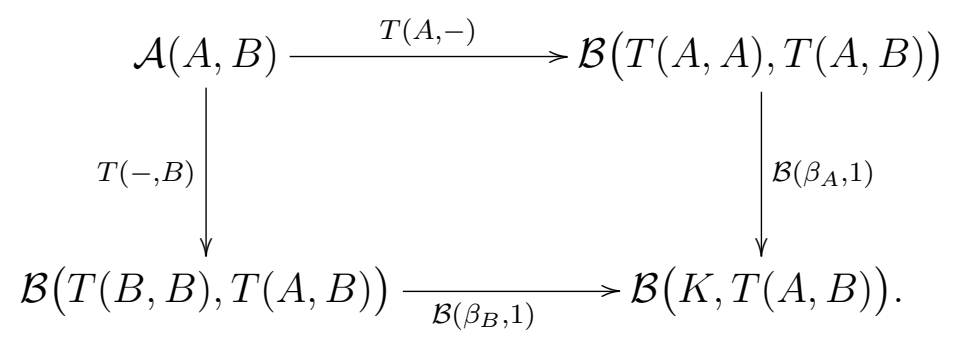

Duality gives the notion, for the same $T$ and $K$, of a $\mathcal{V}$-natural family $\gamma_{A}: T(A, A) \longrightarrow K$; namely the commutativity of

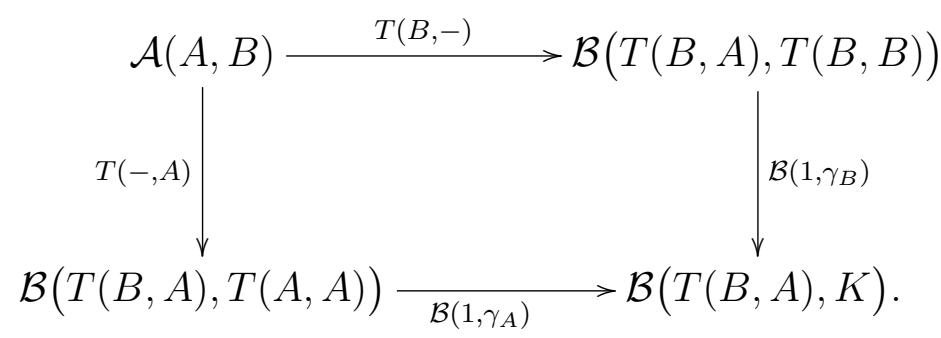

Such extraordinary $\mathcal{V}$-naturality is of course fully alive in the case $\mathcal{V}=\mathbf{S e t}$; it is exhibited there, for instance, by the "naturality in $Y$ " of the $d$ and the $e$ of (1.24). In the case $\mathcal{V}=$ Set, (1.40) and (1.41) have more elementary forms obtained by evaluating them at an arbitrary $f \in \mathcal{A}(A, B)$. Clearly extraordinary $\mathcal{V}$-naturality implies extraordinary Set-naturality: we only have to apply $V$ to (1.40) and (1.41).

Just as for ordinary $\mathcal{V}$-naturality, it is clear that if $\beta_{A}: K \longrightarrow T(A, A)$ is $\mathcal{V}$ natural as above, and if $P: \mathcal{D} \longrightarrow \mathcal{A}$ and $Q: \mathcal{B} \longrightarrow \mathcal{C}$, then the maps $Q \beta_{P D}(=$ $\left.Q_{0} \beta_{P D}\right): Q K \longrightarrow Q T(P D, P D)$ constitute a $\mathcal{V}$-natural family $Q \beta P$.

If $T:(\mathcal{A} \otimes \mathcal{D})^{\mathrm{op}} \otimes(\mathcal{A} \otimes \mathcal{D}) \longrightarrow \mathcal{B}$, a family $\beta_{A D}: K \longrightarrow T(A, D, A, D)$ is $\mathcal{V}$-natural in $(A, D)$ if and only if it is $\mathcal{V}$-natural in $A$ for each fixed $D$ and in $D$ for each fixed $A$, with respect to the partial functors $T(-, D,-, D)$ and $T(A,-, A,-)$ respectively. The most direct proof involves translating (1.40) via (1.32) into a form analogous to (1.7); then, as in the proof for ordinary $\mathcal{V}$-natural transformations, it is a matter of combining (1.21) with (1.3) for $\mathcal{C}$. Thus here, too, $\mathcal{V}$-naturality can be verified variable-by-variable.

That being so, we can combine ordinary and extraordinary $\mathcal{V}$-naturality, and speak of a $\mathcal{V}$-natural family $\alpha_{A B C}: T(A, B, B) \longrightarrow S(A, C, C)$, where $T: \mathcal{A} \otimes \mathcal{B}^{\text {op }} \otimes \mathcal{B} \longrightarrow \mathcal{D}$ and $S: \mathcal{A} \otimes \mathcal{C}^{\text {op }} \otimes \mathcal{C} \longrightarrow \mathcal{D}$. If each of $A, B$ and $C$ stands for a finite number of variables, this is then the most general form.

The analogue of "vertical composition" for such families is the calculus of [25]. There are three basic cases of composability, in addition to that for ordinary $\mathcal{V}$-natural transformations; and two of these three are dual. Each of the three, however, is in fact a sequence of sub-cases indexed by the natural numbers. The reader will see the pattern if we just give the first two sub-cases of the only two essentially-different sequences; the proofs are easy using (1.39)-(1.41).

For $T, S: \mathcal{A}^{\text {op }} \otimes \mathcal{A} \longrightarrow \mathcal{B}$, if $\alpha_{A}: K \longrightarrow T(A, A)$ is $\mathcal{V}$-natural in $A$ and if

$$
\beta_{A B}: T(A, B) \longrightarrow S(A, B)
$$


is $\mathcal{V}$-natural in $\mathcal{A}$ and $\mathcal{B}$, the composite

$$
K \underset{\alpha_{A}}{\longrightarrow} T(A, A) \underset{\beta_{A A}}{\longrightarrow} S(A, A)
$$

is $\mathcal{V}$-natural in $A$. For $T: \mathcal{A}^{\mathrm{op}} \otimes \mathcal{A} \otimes \mathcal{A}^{\mathrm{op}} \otimes \mathcal{A} \longrightarrow \mathcal{B}$ and $S: \mathcal{A}^{\mathrm{op}} \otimes \mathcal{A} \longrightarrow \mathcal{B}$, if $\alpha_{A B}: K \longrightarrow T(A, A, B, B)$ is $\mathcal{V}$-natural in $A$ and $B$ and if

$$
\beta_{A B C}: T(A, B, B, C) \longrightarrow S(A, C)
$$

is $\mathcal{V}$-natural in $A, B$ and $C$, then the composite

$$
K \underset{\alpha_{A A}}{\longrightarrow} T(A, A, A, A) \underset{\beta_{A A A}}{\longrightarrow} S(A, A)
$$

is $\mathcal{V}$-natural in $A$. The next one in this series has $\alpha_{A B C}: K \longrightarrow T(A, A, B, B, C, C)$ and $\beta_{A B C D}: T(A, B, B, C, C, D) \longrightarrow S(A, D)$; and there is of course a dual series.

The self-dual series begins with $T: \mathcal{A} \longrightarrow \mathcal{B}, S: \mathcal{A} \otimes \mathcal{A}^{\text {op }} \otimes \mathcal{A} \longrightarrow \mathcal{B}$, and $R: \mathcal{A} \longrightarrow \mathcal{B}$; if $\alpha_{A B}: T A \longrightarrow S(A, B, B)$ and $\beta_{A B}: S(A, A, B) \longrightarrow R B$ are $\mathcal{V}$-natural in $A$ and $B$, the composite

$$
T A \underset{\alpha_{A A}}{\longrightarrow} S(A, A, A) \underset{\beta_{A A}}{\longrightarrow} R A
$$

is $\mathcal{V}$-natural in $A$. For the same $T$ and $R$, but for $S: \mathcal{A} \otimes \mathcal{A}^{\text {op }} \otimes \mathcal{A} \otimes \mathcal{A}^{\text {op }} \otimes \mathcal{A} \longrightarrow \mathcal{B}$, if $\alpha_{A B C}: T A \longrightarrow S(A, B, B, C, C)$ and $\beta_{A B C}: S(A, A, B, B, C) \longrightarrow R C$ are $\mathcal{V}$-natural in $A, B$ and $C$, then the composite

$$
T A \underset{\alpha_{A A A}}{\longrightarrow} S(A, A, A, A, A) \underset{\beta_{A A A}}{\longrightarrow} R A
$$

is $\mathcal{V}$-natural in $A$.

In each case a handsome geometrical picture is produced if, to the diagrams (1.42)(1.45), there are added curves above the diagram linking variables which must be set equal in $\alpha$, and curves below the diagram linking variables which must be set equal in $\beta$; the geometrical union of these curves, thought of as meeting at the variables, then links the variables which must be set equal in the composite. Of course the associativity of this kind of composition, whenever it makes sense, is a triviality.

\subsection{The $\mathcal{V}$-naturality of the canonical maps}

We turn now to the $\mathcal{V}$-naturality of the canonical maps mentioned at the beginning of 1.7. Of course such an object as $\mathcal{A}(B, C) \otimes \mathcal{A}(A, B)$ is here thought of as the value $\operatorname{Ten}\left(\operatorname{Hom}_{\mathcal{A}}(B, C), \operatorname{Hom}_{\mathcal{A}}(A, B)\right)$ of the appropriate $\mathcal{V}$-functor.

(a) First, it follows at once from (1.29) that $\alpha_{A}: I \longrightarrow \mathcal{B}(T A, S A)$ is $\mathcal{V}$-natural in the sense of (1.40) precisely when $\alpha$ is a $\mathcal{V}$-natural transformation $\alpha: T \rightarrow S$ in the sense of $(1.7)$.

(b) Next, for a $\mathcal{V}$-functor $T: \mathcal{A} \longrightarrow \mathcal{B}$, the map $T_{A B}: \mathcal{A}(A, B) \longrightarrow \mathcal{B}(T A, T B)$ is $\mathcal{V}$-natural in both variables $A$ and $B$. It suffices to verify $\mathcal{V}$-naturality in $B$; and here the appropriate diagram of the form (1.39) reduces, in the light of (1.29), to (1.5) for $T$. 
(c) This last gives, for a $\mathcal{V}$-functor $T: \mathcal{A} \otimes \mathcal{B} \longrightarrow \mathcal{C}$, the $\mathcal{V}$-naturality in $A$ and $A^{\prime}$ of $T(-, B): \mathcal{A}\left(A, A^{\prime}\right) \longrightarrow \mathcal{C}\left(T(A, B), T\left(A^{\prime}, B\right)\right)$. However, this is also $\mathcal{V}$-natural in $B$; the appropriate instance of (1.40) reduces via (1.29) to (1.21).

(d) In particular, for a $\mathcal{V}$-category $\mathcal{A}$, the $\mathcal{A}(A,-)_{B C}: \mathcal{A}(B, C) \longrightarrow[\mathcal{A}(A, B), \mathcal{A}(A, C)]$ of $(1.29)$ is $\mathcal{V}$-natural in all variables.

(e) Composing the $\mathcal{A}(A,-)_{B C}$ of (d) with $g: I \longrightarrow \mathcal{A}(B, C)$ gives, by (a), the $\mathcal{V}$-naturality in $A$ of $\mathcal{A}(A, g): \mathcal{A}(A, B) \longrightarrow \mathcal{A}(A, C)$.

(f) The $\mathcal{V}$-naturality in both variables of $e:[Y, Z] \otimes Y \longrightarrow Z$ is an easy consequence of (1.29) and (1.34).

(g) Since, by (1.29), $M: \mathcal{A}(B, C) \otimes \mathcal{A}(A, B) \longrightarrow \mathcal{A}(A, C)$ for a $\mathcal{V}$-category $\mathcal{A}$ is the composite

$$
\mathcal{A}(B, C) \otimes \mathcal{A}(A, B) \underset{\mathcal{A}(A,-) \otimes 1}{\longrightarrow}[\mathcal{A}(A, B), \mathcal{A}(A, C)] \otimes \mathcal{A}(A, B) \longrightarrow \quad \longrightarrow \mathcal{A}(A, C),
$$

it is $\mathcal{V}$-natural in all variables by (d), (e), (f), (1.42) and (1.44).

(h) The $\mathcal{V}$-naturality of $j_{A}: I \longrightarrow \mathcal{A}(A, A)$ for a $\mathcal{V}$-category $\mathcal{A}$ follows from (a), since $1: 1 \longrightarrow 1: \mathcal{A} \longrightarrow \mathcal{A}$ is $\mathcal{V}$-natural.

(i) The $\mathcal{V}$-naturality in every variable of $a:(X \otimes Y) \otimes Z \longrightarrow X \otimes(Y \otimes Z), l: I \otimes X \longrightarrow X$, $r: X \otimes I \longrightarrow X, c: X \otimes Y \longrightarrow Y \otimes X$, follows easily from (1.34).

(j) From the definition (1.27) of the isomorphism $p$ and the definition (1.34) of Ten(-,Y), we easily see that $p^{-1}:[X,[Y, Z]] \longrightarrow[X \otimes Y, Z]$ is the composite

$$
[X,[Y, Z]] \underset{\operatorname{Ten}(-, Y)}{\longrightarrow}[X \otimes Y,[Y, Z] \otimes Y] \underset{[1, e]}{\longrightarrow}[X \otimes Y, Z] .
$$

It follows from (c), (e), (f), and (1.44) that $p^{-1}$ is $\mathcal{V}$-natural in all variables; whence the same is true of its inverse $p$.

(k) The composite

$$
X \underset{l^{-1}}{\longrightarrow} I \otimes X \underset{j \otimes 1}{\longrightarrow}[X \otimes Y, X \otimes Y] \otimes X \underset{p \otimes 1}{\longrightarrow}[X,[Y, X \otimes Y]] \otimes X \underset{e}{\longrightarrow}[Y, X \otimes Y]
$$

is easily verified to be the $d: X \longrightarrow[Y, X \otimes Y]$ of (1.24); the latter is therefore $\mathcal{V}$-natural in every variable by $(\mathrm{i}),(\mathrm{h}),(\mathrm{j}),(\mathrm{f}),(\mathrm{e}),(1.42)$, and (1.44).

(l) The composite

$$
X \underset{d}{\longrightarrow}[I, X \otimes I] \underset{[1, r]}{\longrightarrow}[I, X]
$$

is the isomorphism $i: X \longrightarrow[I, X]$, which is therefore $\mathcal{V}$-natural. 
This completes the list of canonical maps, and we end this section with a general principle. Since a map $f: X \otimes Y \longrightarrow Z$ and its image $\bar{f}: X \longrightarrow[Y, Z]$ under the $\pi$ of (1.23) are related by $\bar{f}=[1, f] d$ and $f=e(\bar{f} \otimes 1)$, it follows from (f), (k), (e) and (1.42)-(1.44) that, for $\mathcal{V}$-functors $T, S, R$ of suitable variances with codomain $\mathcal{V}$, we have:

(m) A family $f: T(D, D, A, B) \otimes S(E, E, A, C) \longrightarrow R(F, F, B, C)$ is $\mathcal{V}$-natural in any of its variables if and only if the corresponding

$$
\bar{f}: T(D, D, A, B) \longrightarrow[S(E, E, A, C), R(F, F, B, C)]
$$

is so.

\subsection{The (weak) Yoneda lemma for $\mathcal{V}$-CAT}

The form of the Yoneda Lemma we give here is a weak one, in that it asserts a bijection of sets rather than an isomorphism of objects of $\mathcal{V}$; a stronger form will be given in 2.4 below.

Consider a $\mathcal{V}$-functor $F: \mathcal{A} \longrightarrow \mathcal{V}$ and an object $K$ of $\mathcal{A}$. To each $\mathcal{V}$-natural transformation $\alpha: \mathcal{A}(K,-) \longrightarrow F$ we can assign the element $\eta: I \longrightarrow F K$ of $F K$ given by the composite

$$
I \underset{j_{K}}{\longrightarrow} \mathcal{A}(K, K) \underset{\alpha_{K}}{\longrightarrow} F K .
$$

The Yoneda Lemma asserts that this gives a bijection between the $\operatorname{set} \mathcal{V}$-nat $(\mathcal{A}(K,-), F)$ of $\mathcal{V}$-natural transformations and the set $\mathcal{V}_{0}(I, F K)(=V F K)$ of elements of $F K$; the component $\alpha_{A}$ being given in terms of $\eta$ as the composite

$$
\mathcal{A}(K, A) \underset{F_{K A}}{\longrightarrow}[F K, F A] \underset{[\eta, 1]}{\longrightarrow}[I, F A] \underset{i^{-1}}{\longrightarrow} F A .
$$

For the proof, we first note that, for any $\eta,(1.47)$ is indeed $\mathcal{V}$-natural in $A$, by $1.8(\mathrm{~b})$, (e), and (1). Next, if $\alpha$ is defined by (1.47), $\alpha_{K} j_{K}$ is in fact $\eta$; this follows easily from (1.6) for $F$, the Set-naturality of $j$ (a consequence, as we saw in (1.7), of its $\mathcal{V}$-naturality), the naturality of $i$, and the easy verification that $j_{I}=i_{I}: I \longrightarrow[I, I]$. It remains to show that (1.47) is in fact $\alpha_{A}$, when $\eta$ is given by (1.46). In the diagram

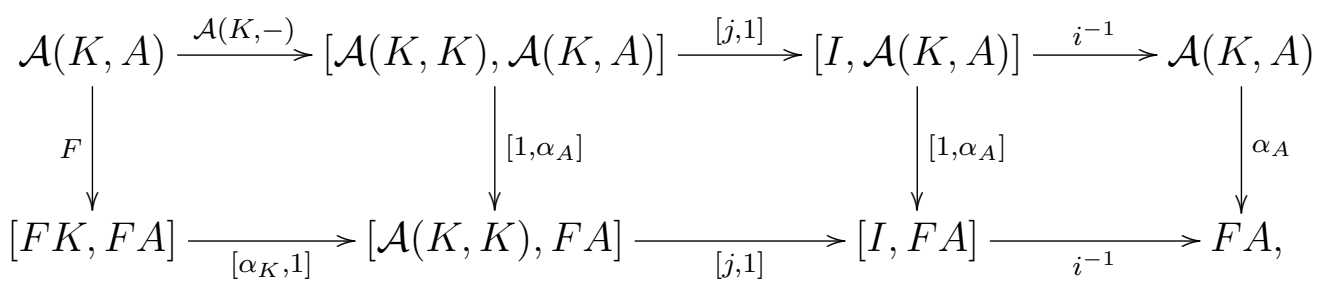

the left square commutes by the $\mathcal{V}$-naturality of $\alpha$ in the form (1.39), the middle square trivially, and the right square by the naturality of $i$. The proof is complete when we observe that the top edge of the diagram is the identity; which follows from the definition (1.29) of $\mathcal{A}(K,-)$, the axiom (1.4) for $\mathcal{A}$, and the definition (1.26) of $i$ in terms of $r$. 
There is an extra-variable form of the result, involving functors $F: \mathcal{B}^{\text {op }} \otimes \mathcal{A} \longrightarrow \mathcal{V}$ and $K: \mathcal{B} \longrightarrow \mathcal{A}$, and a family $\alpha_{B A}: \mathcal{A}(K B, A) \longrightarrow F(B, A)$ which is $\mathcal{V}$-natural in $A$ for each fixed $B$, so that it corresponds as above to a family $\eta_{B}: I \longrightarrow F(B, K B)$. It then follows from (1.46) and (1.42) that $\eta$ is $\mathcal{V}$-natural in $B$ if $\alpha$ is, and from (1.47) and (1.44) that $\alpha$ is $\mathcal{V}$-natural in $B$ if $\eta$ is.

It is worth noting an alternative way of writing (1.47) when $F$ has the form $\mathcal{B}(B, T-)$ for a $\mathcal{V}$-functor $T: \mathcal{A} \longrightarrow \mathcal{B}$, so that $\eta: I \longrightarrow \mathcal{B}(B, T K)$ is then a map $\eta: B \longrightarrow T K$ in $\mathcal{B}_{0}$ (in fact, the image of $1_{K}$ under $\left.V \alpha_{K}: \mathcal{A}_{0}(K, K) \longrightarrow \mathcal{B}_{0}(B, T K)\right)$. Then $\alpha_{A}$ is the composite

$$
\mathcal{A}(K, A) \underset{T_{K A}}{\longrightarrow} \mathcal{B}(T K, T A) \underset{\mathcal{B}(\eta, 1)}{\longrightarrow} \mathcal{B}(B, T A)
$$

which is easily seen by (1.32) to coincide with (1.47). In particular, every $\mathcal{V}$-natural $\alpha: \mathcal{A}(K,-) \longrightarrow \mathcal{A}(L,-)$ is $\mathcal{A}(k,-)$ for a unique $k: L \longrightarrow K$; and clearly $k$ is an isomorphism if and only if $\alpha$ is.

\subsection{Representability of $\mathcal{V}$-functors; the representing object as a $\mathcal{V}$ - functor of the passive variables}

We have called the $\mathcal{V}$-functors $\mathcal{A}(K,-): \mathcal{A} \longrightarrow \mathcal{V}$ representable. More generally, however, a $\mathcal{V}$-functor $F: \mathcal{A} \longrightarrow \mathcal{V}$ is called representable if there is some $K \in \mathcal{A}$ and an isomorphism $\alpha: \mathcal{A}(K,-) \longrightarrow F$. Then the pair $(K, \alpha)$ is a representation of $F$; it is essentially unique if it exists, in the sense that any other representation $\alpha^{\prime}: \mathcal{A}\left(K^{\prime},-\right) \longrightarrow F$ has the form $\alpha^{\prime}=\alpha \cdot \mathcal{A}(k,-)$ for a unique isomorphism $k: K \longrightarrow K^{\prime}$. The corresponding $\eta: I \longrightarrow F K$ is called the unit of the representation. (We call $\eta$ the counit when we are representing a contravariant $F: \mathcal{A}^{\text {op }} \longrightarrow \mathcal{V}$ in the form $\mathcal{A}(-, K)$.)

For a general $\mathcal{V}$, there is no simple criterion in terms of an $\eta: I \longrightarrow F K$ for the corresponding $\alpha: \mathcal{A}(K,-) \longrightarrow F$ to be an isomorphism. It is of course otherwise in the classical case $\mathcal{V}=$ Set; there we have the comma-category $1 / F=\operatorname{el} F$ of "elements of $F$ ", whose objects are pairs $(A, x)$ with $A \in \mathcal{A}$ and $x \in F A$; and $\alpha$ is invertible if and only if $(K, \eta)$ is initial in el $F$, so that $F$ is representable if and only if el $F$ has an initial object. However, any such kind of "universal property" criterion ultimately expresses a bijection of sets, and cannot suffice in general to characterize an isomorphism in $\mathcal{V}_{0}$.

Now let $F: \mathcal{B}^{\text {op }} \otimes \mathcal{A} \longrightarrow \mathcal{V}$ be such that each $F(B,-): \mathcal{A} \longrightarrow \mathcal{V}$ admits a representation $\alpha_{B}: \mathcal{A}(K B,-) \longrightarrow F(B,-)$. Then there is exactly one way of defining $K_{B C}: \mathcal{B}(B, C) \longrightarrow \mathcal{A}(K B, K C)$ that makes $K$ a $\mathcal{V}$-functor for which

$$
\alpha_{B A}: \mathcal{A}(K B, A) \longrightarrow F(B, A)
$$

is $\mathcal{V}$-natural in $B$ as well as $A$.

For by 1.9 , the $\mathcal{V}$-naturality in $B$ of $\alpha_{B A}$ is equivalent to that of $\eta_{B}$, which by $(1.40)$ 
means the commutativity of

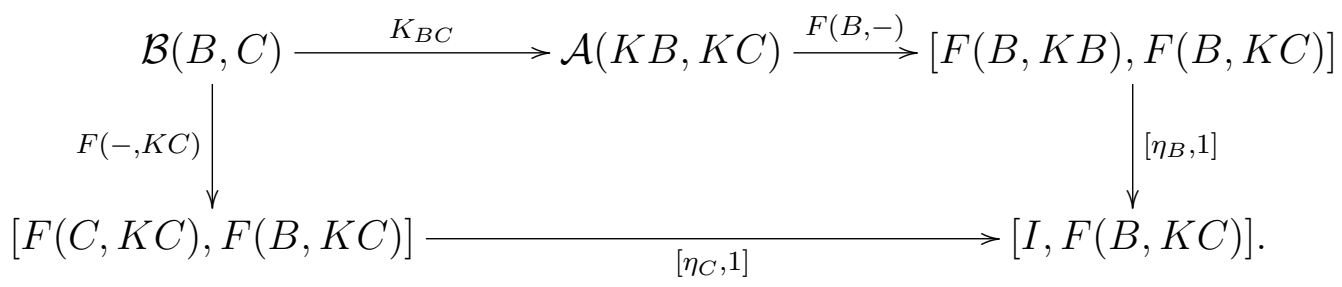

Now the composite $\left[\eta_{B}, 1\right] F(B,-)$ in this diagram is, by $(1.47)$, the composite isomorphism

$$
\mathcal{A}(K B, K C) \underset{\alpha_{B, K C}}{\longrightarrow} F(B, K C) \underset{i}{\longrightarrow}[I, F(B, K C)]
$$

so that (1.49) forces us to define $K_{B C}$ as

$$
K_{B C}=\alpha^{-1} i^{-1}\left[\eta_{C}, 1\right] F(-, K C) .
$$

This composite (1.50) is $\mathcal{V}$-natural in $B$, by 1.7 and 1.8 ; and from this the proof of $1.8(\mathrm{~b})$, read backwards, gives the $\mathcal{V}$-functor axiom (1.5) for $K$; while the remaining $\mathcal{V}$-functor axiom (1.6) is a trivial consequence of (1.49).

\subsection{Adjunctions and equivalences in $\mathcal{V}$-CAT}

As in any 2-category (see [49]) an adjunction $\eta, \epsilon: S \dashv T: \mathcal{A} \longrightarrow \mathcal{B}$ in $\mathcal{V}$-CAT between $T: \mathcal{A} \longrightarrow \mathcal{B}$ (the right adjoint) and $S: \mathcal{B} \longrightarrow \mathcal{A}$ (the left adjoint) consists of $\eta: 1 \longrightarrow T S$ (the unit) and $\epsilon: S T \longrightarrow 1$ (the counit) satisfying the triangular equations $T \epsilon . \eta T=1$ and $\epsilon S . S \eta=1$. By the Yoneda Lemma of 1.9 , such adjunctions in $\mathcal{V}$-CAT are in bijection with $\mathcal{V}$-natural isomorphisms

$$
n: \mathcal{A}(S B, A) \cong \mathcal{B}(B, T A)
$$

for by (1.48) a $\mathcal{V}$-natural map $n: \mathcal{A}(S B, A) \longrightarrow \mathcal{B}(B, T A)$ has the form $n=\mathcal{B}(\eta, 1) T$ for a unique $\eta$, while a $\mathcal{V}$-natural map $\bar{n}: \mathcal{B}(B, T A) \longrightarrow \mathcal{A}(S B, A)$ has the form $\bar{n}=\mathcal{A}(1, \epsilon) S$ for a unique $\epsilon$, and the equations $\bar{n} n=1$ and $n \bar{n}=1$ reduce by Yoneda precisely to the triangular equations.

The 2-functor $(-)_{0}: \mathcal{V}$-CAT $\longrightarrow$ CAT carries such an adjunction into an ordinary adjunction $\eta, \epsilon: S_{0} \dashv T_{0}: \mathcal{A}_{0} \longrightarrow \mathcal{B}_{0}$ in CAT. It is immediate from (1.33) that the corresponding isomorphism of hom-sets is

$$
V n: \mathcal{A}_{0}(S B, A) \cong \mathcal{B}_{0}(B, T A)
$$

Note that the unit and counit of this ordinary adjunction are the same $\eta$ and $\epsilon$, now seen as natural rather than $\mathcal{V}$-natural.

By 1.10, a $\mathcal{V}$-functor $T: \mathcal{A} \longrightarrow \mathcal{B}$ has a left adjoint (which is then unique to within isomorphism, as in any 2-category) exactly when each $\mathcal{B}(B, T-)$ is representable. More generally, if those $B$ for which $\mathcal{B}(B, T-)$ is representable constitute the full subcategory $\mathcal{B}^{\prime}$ of $\mathcal{B}$, we get a $\mathcal{V}$-functor $S: \mathcal{B}^{\prime} \longrightarrow \mathcal{A}$ called a partial left adjoint to $T$. 
When $V$ is conservative - faithfulness is not enough - the existence of a left adjoint $S_{0}$ for $T_{0}$ implies that of a left adjoint $S$ for $T$ : we set $S B=S_{0} B$, define a map $n$ that is $\mathcal{V}$-natural in $A$ by $n=\mathcal{B}(\eta, 1) T$ as above (using the unit $\eta$ for $S_{0} \dashv T_{0}$ ), and deduce that $n$ is an isomorphism since the $V n$ of (1.52) is. Thus, taking $\mathcal{V}=\mathbf{A b}$ for instance, an additive functor has a left adjoint exactly when its underlying functor has one.

Again by 1.10, in the extra-variable case of a $\mathcal{V}$-functor $T: \mathcal{C}^{\mathrm{op}} \otimes \mathcal{A} \longrightarrow \mathcal{B}$, if each $T(C,-)$ has a left adjoint $S(-, C)$, then $S$ automatically becomes a $\mathcal{V}$-functor $\mathcal{B} \otimes \mathcal{C} \longrightarrow \mathcal{A}$ in such a way that $n: \mathcal{A}(S(B, C), A) \cong \mathcal{B}(B, T(C, A))$ is $\mathcal{V}$-natural in all three variables. An evident example, with $\mathcal{A}=\mathcal{B}=\mathcal{C}=\mathcal{V}$, is the adjunction $p:[X \otimes Y, Z] \cong[X,[Y, Z]]$ of (1.27).

For a typical adjunction (1.51) as above, we have a commutative diagram

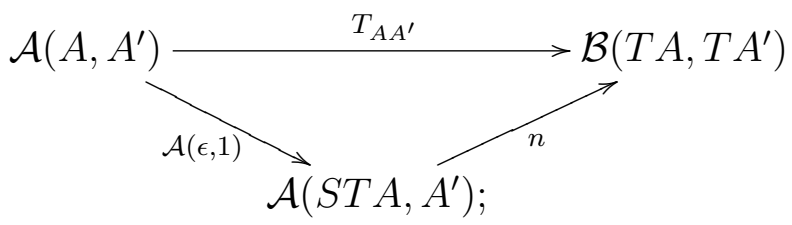

this follows from Yoneda on setting $A^{\prime}=A$ and composing both legs with $j$, since $(V n)\left(\epsilon_{A}\right)=1$. Because $n$ is an isomorphism, we conclude that $T$ is fully faithful if and only if $\epsilon$ is an isomorphism. In consequence, a right adjoint $T$ is fully faithful exactly when $T_{0}$ is so.

As in any 2-category, a $\mathcal{V}$-functor $T: \mathcal{A} \longrightarrow \mathcal{B}$ is called an equivalence, and we write $T: \mathcal{A} \simeq \mathcal{B}$, if there is some $S: \mathcal{B} \longrightarrow \mathcal{A}$ and isomorphisms $\eta: 1 \cong T S$ and $\rho: S T \cong 1$. Replacing $\rho$ (still in any 2-category) by the isomorphism $\epsilon: S T \cong 1$ given by $\epsilon=$ $\rho . S \eta^{-1} T . \rho^{-1} S T$, we actually have an adjunction $\eta, \epsilon: S \dashv T: \mathcal{A} \longrightarrow \mathcal{B}$. The word "equivalence" is sometimes used to mean an adjunction in which, as here $\eta$ and $\epsilon$ are isomorphisms; we write $S \dashv T: \mathcal{A} \simeq \mathcal{B}$.

In the 2-category $\mathcal{V}$-CAT, $T: \mathcal{A} \longrightarrow \mathcal{B}$ is an equivalence if and only if $T$ is fully faithful and essentially surjective on objects; the latter means that every $B \in \mathcal{B}$ is isomorphic (in $\mathcal{B}_{0}$, of course) to $T A$ for some $A \in \mathcal{A}$; we often omit the words "on objects".

For, if $T$ is an equivalence, $T$ is fully faithful since $\epsilon$ is an isomorphism, and is essentially surjective on objects since $\eta_{B}: B \longrightarrow T S B$ is an isomorphism. As for the converse, we choose for each $B \in \mathcal{B}$ an $S B \in \mathcal{A}$ and an isomorphism $\eta_{B}: B \longrightarrow T S B$. The corresponding $\mathcal{V}$-natural-in- $A$ transformation $n=\mathcal{B}\left(\eta_{B}, 1\right) T: \mathcal{A}(S B, A) \longrightarrow \mathcal{B}(B, T A)$ is then an isomorphism because $T$ is fully faithful, so that $S$ becomes automatically a $\mathcal{V}$-functor left adjoint to $T$. Since $\eta$ is an isomorphism, so is $T \epsilon$, by the triangular equation $T \epsilon . \eta T=1$; whence $\epsilon$ is an isomorphism by (1.12), since a fully-faithful $T_{0}$ is certainly conservative.

In any 2-category (see [49]) adjunctions can be composed, forming a category, of which the equivalences are a subcategory. It follows from Yoneda that the composition of adjunctions in $\mathcal{V}$-CAT corresponds to the composition of the $\mathcal{V}$-natural isomorphisms in

$$
\mathcal{A}\left(S S^{\prime} B, A\right) \cong \mathcal{B}\left(S^{\prime} B, T A\right) \cong \mathcal{B}\left(B, T^{\prime} T A\right) .
$$

It is further true in any 2-category (see [49]) that, given adjunctions $\eta, \epsilon: S \dashv$ $T: \mathcal{A} \longrightarrow \mathcal{B}$ and $\eta^{\prime}, \epsilon^{\prime}: S^{\prime} \dashv T^{\prime}: \mathcal{A}^{\prime} \longrightarrow \mathcal{B}^{\prime}$ and 1-cells $P: \mathcal{A} \longrightarrow \mathcal{A}^{\prime}$ and $Q: \mathcal{B} \longrightarrow \mathcal{B}^{\prime}$, there 
is a bijection (with appropriate naturality properties) between 2-cells $\lambda: Q T \longrightarrow T^{\prime} P$ and 2-cells $\mu: S^{\prime} Q \longrightarrow P S$. In $\mathcal{V}$-CAT it follows from Yoneda that $\lambda$ and $\mu$ determine one another through the commutativity of

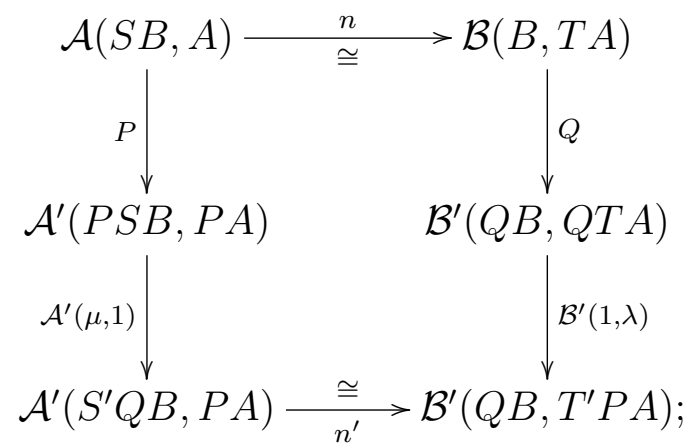

such a pair $\lambda, \mu$ may be called mates.

In particular, when $\mathcal{A}^{\prime}=\mathcal{A}$ with $P=1$ and $\mathcal{B}^{\prime}=\mathcal{B}$ with $Q=1$, so that $S \dashv T$ and $S^{\prime} \dashv T^{\prime}$ are both adjunctions $\mathcal{A} \longrightarrow \mathcal{B}$, we have a bijection between the $\lambda: T \longrightarrow T^{\prime}$ and the $\mu: S^{\prime} \longrightarrow S$. The adjunctions become a 2-category when we define a 2-cell from $S \dashv T$ to $S^{\prime} \dashv T^{\prime}$ to be such a pair $(\lambda, \mu)$.

Any $T: \mathcal{A} \longrightarrow \mathcal{B}$ determines two full subcategories of $\mathcal{B}$; the full image $\mathcal{A}^{\prime}$ of $T$, determined by those $B$ of the form $T A$, and the replete image $\mathcal{A}^{\prime \prime}$ of $T$, determined by those $B$ isomorphic to some $T A$. Clearly $\mathcal{A}^{\prime}$ and $\mathcal{A}^{\prime \prime}$ are equivalent; and each is equivalent to $\mathcal{A}$ if $T$ is fully faithful. A full subcategory which contains all the isomorphs of its objects is said to be replete; any full subcategory has a repletion, namely the replete image of its inclusion. Clearly a fully-faithful $T: \mathcal{A} \longrightarrow \mathcal{B}$ has a left adjoint if and only if the inclusion $\mathcal{A}^{\prime} \longrightarrow \mathcal{B}$ has one, and if and only if the inclusion $\mathcal{A}^{\prime \prime} \longrightarrow \mathcal{B}$ has one.

A full subcategory $\mathcal{A}$ of $\mathcal{B}$ is called reflective if the inclusion $T: \mathcal{A} \longrightarrow \mathcal{B}$ has a left adjoint. This implies, of course, that $\mathcal{A}_{0}$ is reflective in $\mathcal{B}_{0}$, but is in general strictly stronger. It follows - since it is trivially true for ordinary categories - that every retract (in $\mathcal{B}_{0}$ ) of an object of the reflective $\mathcal{A}$ lies in the repletion of $\mathcal{A}$.

When $\mathcal{A}$ is reflective we may so choose the left adjoint $S: \mathcal{B} \longrightarrow A$ that $\epsilon: S T \longrightarrow 1$ is the identity. Then $\eta: 1 \longrightarrow R=T S: \mathcal{B} \longrightarrow \mathcal{B}$ satisfies $R^{2}=R, \eta R=R \eta=1$. Such an $(R, \eta)$ is called an idempotent monad on $\mathcal{B}$. Conversely, for any idempotent monad $(R, \eta)$, both its full image and its replete image are reflective in $\mathcal{B}$. If $R$ is formed as above, the full image is $\mathcal{A}$ again; but $(R, \eta)$ is not uniquely determined by $\mathcal{A}$. The replete image of $R$ is the repletion of $\mathcal{A}$; and there is an evident bijection between full replete reflective subcategories of $\mathcal{B}$ and isomorphism classes of idempotent monads on $\mathcal{B}$. 


\section{Chapter 2}

\section{Functor categories}

\subsection{Ends in $\mathcal{V}$}

So far we have supposed that $\mathcal{V}$ is symmetric monoidal closed, and that the underlying ordinary category $\mathcal{V}_{0}$ is locally small. Henceforth we add the assumption that $\mathcal{V}_{0}$ is complete, in the sense that it admits all small limits; and from 2.5 on, we shall suppose as well that $\mathcal{V}_{0}$ is cocomplete.

Consider a $\mathcal{V}$-functor $T: \mathcal{A}^{\mathrm{op}} \otimes \mathcal{A} \longrightarrow \mathcal{V}$. If there exists a universal $\mathcal{V}$-natural family $\lambda_{A}: K \longrightarrow T(A, A)$, in the sense that every $\mathcal{V}$-natural $\alpha_{A}: X \longrightarrow T(A, A)$ is given by $\alpha_{A}=\lambda_{A} f$ for a unique $f: X \longrightarrow K$, we call $(K, \lambda)$ the end of $T$; it is clearly unique to within a unique isomorphism when it exists. We write $\int_{A \in \mathcal{A}} T(A, A)$ for the object $K$, and by the usual abuse of language also call this object the end of $T$ : then the universal $\mathcal{V}$-natural $\lambda_{A}: \int_{A} T(A, A) \longrightarrow T(A, A)$ may be called the counit of this end.

The $\mathcal{V}$-naturality condition (1.40), in the case $\mathcal{B}=\mathcal{V}$, transforms under the adjunction $\mathcal{V}_{0}(X,[Y, Z]) \cong \mathcal{V}_{0}(X \otimes Y, Z) \cong \mathcal{V}_{0}(Y,[X, Z])$ into

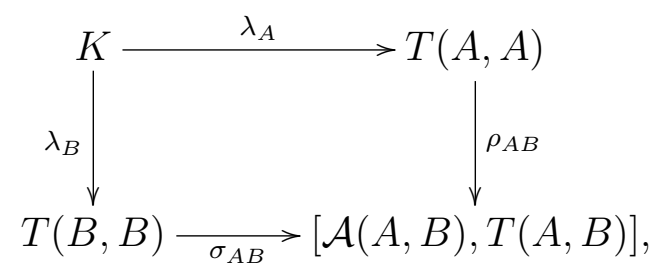

where $\rho_{A B}$ and $\sigma_{A B}$ are the transforms of $T(A,-)_{A B}$ and $T(-, B)_{B A}$. It follows that, when $\mathcal{A}$ is small, the end $\int_{A \in \mathcal{A}} T(A, A)$ certainly exists, and is given as an equalizer

$$
\int_{A \in \mathcal{A}} T(A, A) \underset{\lambda}{\longrightarrow} \prod_{A \in \mathcal{A}} T(A, A) \underset{\sigma}{\stackrel{\rho}{\longrightarrow}} \prod_{A, B \in \mathcal{A}}[\mathcal{A}(A, B), T(A, B)] .
$$

If $\mathcal{A}$, while not necessarily small, is equivalent to a small $\mathcal{V}$-category, then the full image in $\mathcal{A}$ of the latter is a small full subcategory $\mathcal{A}^{\prime}$ for which the inclusion $\mathcal{A}^{\prime} \longrightarrow \mathcal{A}$ is an equivalence. Since it clearly suffices to impose (2.1) only for $A, B \in \mathcal{A}^{\prime}$, it follows that $\int_{A \in \mathcal{A}} T(A, A)$ still exists, being $\int_{A \in \mathcal{A}^{\prime}} T(A, A)$. In consequence, we henceforth change the meaning of "small", extending it to include those $\mathcal{V}$-categories equivalent to small ones in the sense of 1.2. 
The universal property of the end - expressed, as it stands, by a bijection of sets - in fact lifts to an isomorphism in $\mathcal{V}_{0}$; in the sense that

$$
\left[X, \lambda_{A}\right]:\left[X, \int_{A} T(A, A)\right] \longrightarrow[X, T(A, A)]
$$

expresses its domain as the end of its codomain:

$$
\left.\left[X, \int_{A} T(A, A)\right] \cong \int_{A}[X, T(A, A)] \quad \text { (with counit }\left[X, \lambda_{A}\right]\right) .
$$

For $Y \longrightarrow[X, T(A, A)]$ is $\mathcal{V}$-natural if and only if $Y \otimes X \longrightarrow T(A, A)$ is, by $1.8(\mathrm{~m})$. Note that the right side of (2.3) exists for all $X$ if $\int_{A} T(A, A)$ exists; the converse of this is also true, as we see on taking $X=I$.

If $\alpha: T \longrightarrow T^{\prime}: \mathcal{A}^{\mathrm{op}} \otimes \mathcal{A} \longrightarrow \mathcal{V}$, and if the ends of $T$ and $T^{\prime}$ exist, the top leg of the diagram

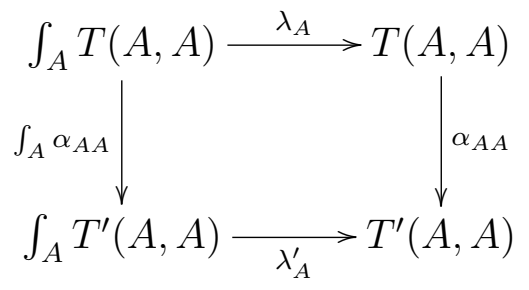

is $\mathcal{V}$-natural by (1.42); so that, by the universal property of $\lambda^{\prime}$, there is a unique $\int_{A} \alpha_{A A}$ rendering the diagram commutative.

This "functoriality" of ends lifts, in the appropriate circumstances, to a $\mathcal{V}$-functoriality. Suppose namely that, for $T: \mathcal{A}^{\text {op }} \otimes \mathcal{A} \otimes \mathcal{B} \longrightarrow \mathcal{V}$, the end $H B=\int_{A} T(A, A, B)$ exists for each $B$. Then there is exactly one way of making $H$ into a $\mathcal{V}$-functor $H: \mathcal{B} \longrightarrow \mathcal{V}$ that renders $\lambda_{A B}: H B \longrightarrow T(A, A, B) \mathcal{V}$-natural in $B$ as well as in $A$.

For, by (1.39), the $\mathcal{V}$-naturality in $B$ is expressed by the commutativity of

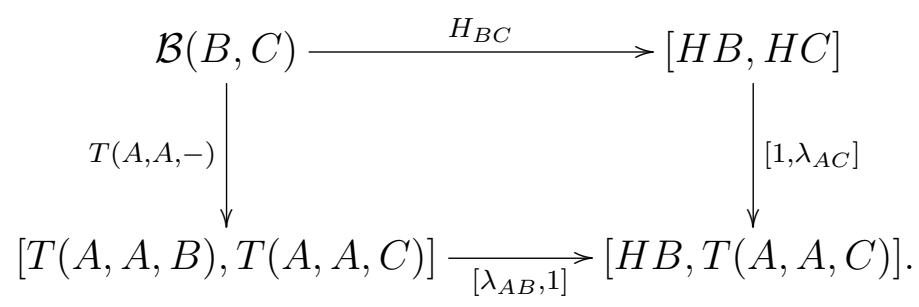

The bottom leg is $\mathcal{V}$-natural in $A$ by $1.8(\mathrm{c}), 1.8(\mathrm{e})$, and (1.43). The right edge is itself an end by (2.3). Hence a unique $H_{B C}$ renders the diagram commutative. The $\mathcal{V}$-functor axioms (1.5) and (1.6) for $H$ then follow from the $\mathcal{V}$-naturality of $M$ and $j(1.8(\mathrm{~g})$ and (h)), the composition-calculus of 1.7 , and the universal property of the counit $[1, \lambda]$.

In these circumstances, if $R: \mathcal{B} \longrightarrow \mathcal{V}$, a family $\beta_{B}: R B \longrightarrow \int_{A} T(A, A, B)$ is $\mathcal{V}$-natural in $B$ if and only if the composite

$$
R B \underset{\beta_{B}}{\longrightarrow} \int_{A} T(A, A, B) \underset{\lambda_{A B}}{\longrightarrow} T(A, A, B)
$$

is so. One direction is trivial, while the other follows easily from (2.5) and the universal property of the counit $[1, \lambda]$. In fact, this result can also be seen - using $1.8(\mathrm{~m})$ to view 
$(2.6)$ as a $\mathcal{V}$-natural family $I \longrightarrow[R B, T(A, A, B)]$ and using $(2.3)$ - as a special case of the following.

Let $T:(\mathcal{A} \otimes \mathcal{B})^{\mathrm{op}} \otimes(\mathcal{A} \otimes \mathcal{B}) \longrightarrow \mathcal{V}$, recalling that $(\mathcal{A} \otimes \mathcal{B})^{\mathrm{op}}=\mathcal{A}^{\mathrm{op}} \otimes \mathcal{B}^{\mathrm{op}}$. Suppose that, for each $B, C \in \mathcal{B}$, the end $\int_{A \in \mathcal{A}} T(A, B, A, C)$ exists; then, as above, it is the value of a $\mathcal{V}$-functor $\mathcal{B}^{\text {op }} \otimes \mathcal{B} \longrightarrow \mathcal{V}$. Every family $\alpha_{A B}: X \longrightarrow T(A, B, A, B)$ that is $\mathcal{V}$-natural in $A$ factorizes uniquely as

$$
X \underset{\beta_{B}}{\longrightarrow} \int_{A \in \mathcal{A}} T(A, B, A, B) \underset{\lambda_{A B B}}{\longrightarrow} T(A, B, A, B) ;
$$

and it follows again from (2.5) and the universal property of $[1, \lambda]$ that the $\mathcal{V}$-naturality in $B$ of $\alpha_{A B}$ is equivalent to the $\mathcal{V}$-naturality in $B$ of $\beta_{B}$.

Since, by 1.7, $\mathcal{V}$-naturality of $\alpha_{A B}$ in $A$ and $B$ separately coincides with its $\mathcal{V}$-naturality in $(A, B) \in \mathcal{A} \otimes \mathcal{B}$, we deduce the Fubini Theorem: if every $\int_{A} T(A, B, A, C)$ exists, then

$$
\int_{(A, B) \in \mathcal{A} \otimes \mathcal{B}} T(A, B, A, B) \cong \int_{B \in \mathcal{B}} \int_{A \in \mathcal{A}} T(A, B, A, B),
$$

either side existing if the other does.

This in turn gives, since $\mathcal{A} \otimes \mathcal{B} \cong \mathcal{B} \otimes \mathcal{A}$, the following interchange of ends theorem, often itself called the Fubini Theorem: if every $\int_{A} T(A, B, A, C)$ and every $\int_{B} T(A, B, D, B)$ exists, then

$$
\int_{A \in \mathcal{A}} \int_{B \in \mathcal{B}} T(A, B, A, B) \cong \int_{B \in \mathcal{B}} \int_{A \in \mathcal{A}} T(A, B, A, B),
$$

either side existing if the other does.

\subsection{The functor-category $[\mathcal{A}, \mathcal{B}]$ for small $\mathcal{A}$}

For $\mathcal{V}$-functors $T, S: \mathcal{A} \longrightarrow \mathcal{B}$ we introduce the notation

$$
[\mathcal{A}, \mathcal{B}](T, S)=\int_{A \in \mathcal{A}} \mathcal{B}(T A, S A)
$$

for the end on the right, whenever it exists; and we write the counit as

$$
E_{A}=E_{A, T S}:[\mathcal{A}, \mathcal{B}](T, S) \longrightarrow \mathcal{B}(T A, S A) .
$$

We write $[\mathcal{A}, \mathcal{B}]_{0}(T, S)$ for the set $V[\mathcal{A}, \mathcal{B}](T, S)$ of elements $\alpha: I \longrightarrow[\mathcal{A}, \mathcal{B}](T, S)$ of this end. These of course correspond to $\mathcal{V}$-natural families

$$
\alpha_{A}=E_{A} \alpha: I \longrightarrow \mathcal{B}(T A, S A),
$$

which by 1.8 (a) are precisely the $\mathcal{V}$-natural transformations $\alpha: T \longrightarrow S: \mathcal{A} \longrightarrow \mathcal{B}$.

Note that, by (2.3) and the adjunction $[X,[Y, Z]] \cong[Y,[X, Z]]$, we have

$$
[X,[\mathcal{A}, \mathcal{V}](T, S)] \cong[\mathcal{A}, \mathcal{V}](T,[X, S-]),
$$

either side existing for all $X \in \mathcal{V}$ if the other does.

When $[\mathcal{A}, \mathcal{B}](T, S)$ exists for all $T, S: \mathcal{A} \longrightarrow \mathcal{B}$, as it surely does by 2.1 if $\mathcal{A}$ is small, it is the hom-object of a $\mathcal{V}$-category $[\mathcal{A}, \mathcal{B}]$ with all the $T: \mathcal{A} \longrightarrow \mathcal{B}$ as objects. The composition 
law of $[\mathcal{A}, \mathcal{B}]$ is, by $1.8(\mathrm{~g}),(1.43)$, and the universal property of $E_{A, T R}$, uniquely determined by the commutativity of

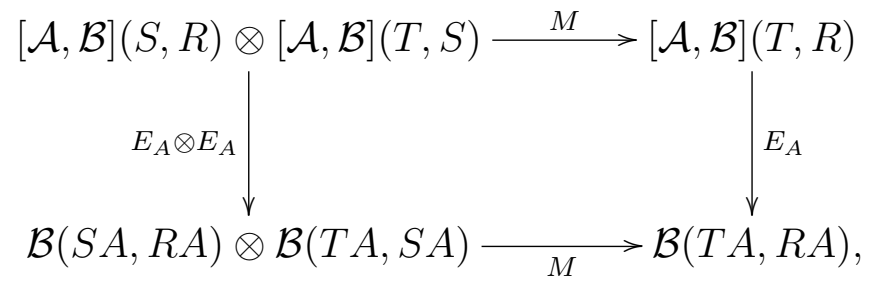

and its identity elements are similarly determined by

$$
E_{A} j_{T}=j_{T A}
$$

the axioms (1.3) and (1.4) follow at once from the corresponding axioms for $\mathcal{B}$ and the universal property of $E_{A}$.

We call $[\mathcal{A}, \mathcal{B}]$ the functor category; its underlying category $[\mathcal{A}, \mathcal{B}]_{0}$ is by $(2.12)$ the ordinary category of all $\mathcal{V}$-functors $\mathcal{A} \longrightarrow \mathcal{B}$ and all $\mathcal{V}$-natural transformations between them - that is

$$
[\mathcal{A}, \mathcal{B}]_{0}=\mathcal{V}-\operatorname{CAT}(\mathcal{A}, \mathcal{B})
$$

Even when $[\mathcal{A}, \mathcal{B}]$ does not exist, it may sometimes be convenient to use $[\mathcal{A}, \mathcal{B}]_{0}$ as an abbreviation for $\mathcal{V}$-CAT $(\mathcal{A}, \mathcal{B})$.

When $[\mathcal{A}, \mathcal{B}]$ exists, the function $T \mapsto T A$ and the maps $E_{A, T S}$ of $(2.11)$ constitute, by (2.14) and (2.15), a $\mathcal{V}$-functor $E_{A}:[\mathcal{A}, \mathcal{B}] \longrightarrow \mathcal{B}$, called evaluation at $A$. By (2.12), the ordinary functor underlying $E_{A}$ sends a map $\alpha \in[\mathcal{A}, \mathcal{B}]_{0}(T, S)$ to its component $\alpha_{A}$.

There is a $\mathcal{V}$-functor $E:[\mathcal{A}, \mathcal{B}] \otimes \mathcal{A} \longrightarrow \mathcal{B}$, simply called evaluation, whose partial functors are given by

$$
E(-, A)=E_{A}:[\mathcal{A}, \mathcal{B}] \longrightarrow \mathcal{B}, \quad E(T,-)=T: \mathcal{A} \longrightarrow \mathcal{B} .
$$

To show this we must verify the appropriate instance of (1.21); but, by the proof of $1.8(\mathrm{c})$ read backwards, this instance reduces via (1.29) to the instance of (1.40) expressing the $\mathcal{V}$-naturality in $A$ of $E_{A, T S}$.

More generally, $[\mathcal{A}, \mathcal{B}](T, S)$ may exist, not for all $T, S$, but for all those in some subset of the set of $\mathcal{V}$-functors $\mathcal{A} \longrightarrow \mathcal{B}$; a typical example would be the subset of those $\mathcal{A} \longrightarrow \mathcal{B}$ which are left Kan extensions, in the sense of 4.1 below, of their restrictions to some fixed small full subcategory of $\mathcal{A}$. Then we get as above a $\mathcal{V}$-category $[\mathcal{A}, \mathcal{B}]^{\prime}$ with these functors as objects, whose underlying category $[\mathcal{A}, \mathcal{B}]_{0}^{\prime}$ is the corresponding full subcategory of $\mathcal{V}-\operatorname{CAT}(\mathcal{A}, \mathcal{B})$; and we have the evaluation functor $E:[\mathcal{A}, \mathcal{B}]^{\prime} \otimes \mathcal{A} \longrightarrow \mathcal{B}$.

Still more generally, we may wish to consider $[\mathcal{A}, \mathcal{B}](T, S)$ just for certain particular pairs $T, S$ for which it exists - as in the Yoneda isomorphism of 2.4 below. To discuss its functoriality in this generality, suppose we have functors $P: \mathcal{C} \otimes \mathcal{A} \longrightarrow \mathcal{B}$ and $Q: \mathcal{D} \otimes \mathcal{A} \longrightarrow \mathcal{B}$ such that $[\mathcal{A}, \mathcal{B}](P(C,-), Q(D,-))$ exists for each $C$ and $D$. Then there is, by 2.1 , a unique $\mathcal{V}$-functor $H: \mathcal{C}^{\mathrm{op}} \otimes \mathcal{D} \longrightarrow \mathcal{V}$ having

$$
H(C, D)=[\mathcal{A}, \mathcal{B}](P(C,-), Q(D,-)),
$$


with respect to which $E_{A}:[\mathcal{A}, \mathcal{B}](P(C,-), Q(D,-)) \longrightarrow \mathcal{B}(P(C, A), Q(D, A))$ is $\mathcal{V}$-natural in $C$ and $D$ as well as $A$. Moreover by (2.6) and (2.7), in the situations (the second of which has $\mathcal{D}=\mathcal{C}$ )
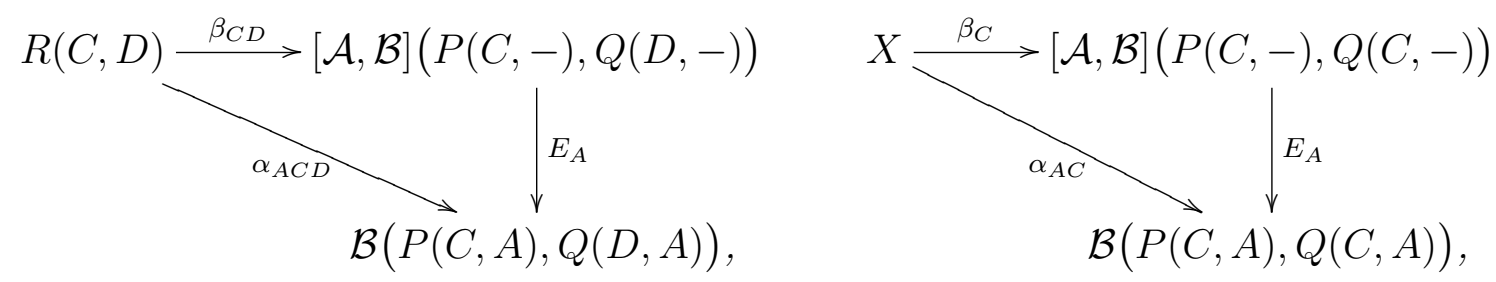

$\alpha$ is $\mathcal{V}$-natural in $\mathcal{C}$ or $\mathcal{D}$ if and only if $\beta$ is.

When $[\mathcal{A}, \mathcal{B}]$, or even some $[\mathcal{A}, \mathcal{B}]^{\prime}$, exists, the $H$ of $(2.18)$ can be given more explicitly see 2.3 below.

\subsection{The isomorphism $[\mathcal{A} \otimes \mathcal{B}, \mathcal{C}] \cong[\mathcal{A},[\mathcal{B}, \mathcal{C}]]$}

We return to the case where $[\mathcal{A}, \mathcal{B}]$ exists. The $\mathcal{V}$-functor $E:[\mathcal{A}, \mathcal{B}] \otimes \mathcal{A} \longrightarrow \mathcal{B}$ induces, for each $\mathcal{V}$-category $\mathcal{C}$, an ordinary functor

$$
\mathcal{V}-\operatorname{CAT}(\mathcal{C},[\mathcal{A}, \mathcal{B}]) \longrightarrow \mathcal{V}-\operatorname{CAT}(\mathcal{C} \otimes \mathcal{A}, \mathcal{B})
$$

sending $G: \mathcal{C} \longrightarrow[\mathcal{A}, \mathcal{B}]$ to the composite

$$
\mathcal{C} \otimes \mathcal{A} \underset{G \otimes 1}{\longrightarrow}[\mathcal{A}, \mathcal{B}] \otimes \mathcal{A} \underset{E}{\longrightarrow} \mathcal{B},
$$

and sending $\beta: G \longrightarrow G^{\prime}: \mathcal{C} \longrightarrow[\mathcal{A}, \mathcal{B}]$ to the composite $E\left(\beta \otimes 1_{\mathcal{A}}\right)$. Clearly the map (2.20) is 2-natural in $\mathcal{C}$; and in fact it is an isomorphism of categories.

To prove this we must first show that every $P: \mathcal{C} \otimes \mathcal{A} \longrightarrow \mathcal{B}$ has the form (2.21) for a unique $G$. Equating the partial functors of $P$ and (2.21) gives

$$
P(C,-)=G C, \quad P(-, A)=E_{A} G .
$$

The first of these determines $G$ on objects, whereupon the second asserts the commutativity of

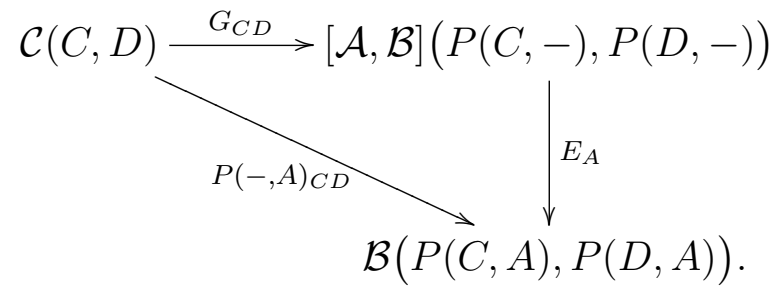

Since $P(-, A)_{C D}$ is $\mathcal{V}$-natural in $A$ by $1.8(\mathrm{c})$, this determines a unique $G_{C D}$ by the universal property of $E_{A}$. By this same universal property together with the compositioncalculus of 1.7, the $\mathcal{V}$-functor axioms (1.5) and (1.6) for $G$ follow at once from those for $P(-, A)$. 
Before showing that (2.20) is bijective on maps as well as on objects, let us analyze the $\mathcal{V}$-functoriality $(2.18)$ when $[\mathcal{A}, \mathcal{B}]$ exists. If $P: \mathcal{C} \otimes \mathcal{A} \longrightarrow \mathcal{B}$ and $Q: \mathcal{D} \otimes \mathcal{A} \longrightarrow \mathcal{B}$ correspond by $(2.20)$ to $G: \mathcal{C} \longrightarrow[\mathcal{A}, \mathcal{B}]$ and $F: \mathcal{D} \longrightarrow[\mathcal{A}, \mathcal{B}]$, we have the composite $\mathcal{V}$-functor

$$
\mathcal{C}^{\mathrm{op}} \otimes \mathcal{D} \underset{G^{\mathrm{op}} \otimes F}{\longrightarrow}[\mathcal{A}, \mathcal{B}]^{\mathrm{op}} \otimes[\mathcal{A}, \mathcal{B}] \underset{\operatorname{Hom}_{[\mathcal{A}, \mathcal{B}]}}{\longrightarrow} \mathcal{V}
$$

and with respect to this, $E_{A}:[\mathcal{A}, \mathcal{B}](G C, F D) \longrightarrow \mathcal{B}\left(E_{A} G C, E_{A} F D\right)$ is $\mathcal{V}$-natural in $\mathcal{C}$ and $\mathcal{D}$, by 1.7 and $1.8(\mathrm{~b})$. By $(2.22)$, this $E_{A}$ is

$$
E_{A}:[\mathcal{A}, \mathcal{B}](P(C,-), Q(D,-)) \rightarrow \mathcal{B}(P(C, A), Q(D, A)) ;
$$

hence the $H$ of (2.18), by its uniqueness, must be (2.24).

To complete the proof that (2.20) is an isomorphism of categories, it remains to verify that, if $P$ and $P^{\prime}$ are the images under (2.20) of $G$ and $G^{\prime}$, every $\alpha: P \longrightarrow P^{\prime}$ is $E\left(\beta \otimes 1_{\mathcal{A}}\right)$ for a unique $\beta: G \longrightarrow G^{\prime}$. By $(2.22)$ the equation $\alpha=E\left(\beta \otimes 1_{\mathcal{A}}\right)$ has the component-form $\alpha_{C A}=E_{A} \beta_{C}$, which by $(2.12)$ is

$$
\alpha_{C A}=E_{A} \beta_{C}=\left(\beta_{C}\right)_{A} .
$$

Thus $\beta_{C}: G C \longrightarrow G^{\prime} C$ must be the $\mathcal{V}$-natural transformation $\alpha_{C-}: P(C,-) \longrightarrow P^{\prime}(C,-)$; and the proof will be complete when we show that $\beta_{C}$, so defined, is $\mathcal{V}$-natural in $C$. If we look on $\beta_{C}$ as a map $I \longrightarrow[\mathcal{A}, \mathcal{B}]\left(G C, G^{\prime} C\right)$, this follows from $1.8(\mathrm{~m})$ and $(2.19)$, now that $H$ is identified as $(2.24)$.

More generally, if $[\mathcal{A}, \mathcal{B}]$ does not exist but some full subcategory $[\mathcal{A}, \mathcal{B}]^{\prime}$ does, in the sense of 2.2 , we have by the same arguments an isomorphism between the category $\mathcal{V}$-CAT $\left(\mathcal{C},[\mathcal{A}, \mathcal{B}]^{\prime}\right)$ and the full subcategory of $\mathcal{V}$-CAT $(\mathcal{C} \otimes \mathcal{A}, \mathcal{B})$ determined by those $P: \mathcal{C} \otimes \mathcal{A} \longrightarrow \mathcal{B}$ for which each $P(C,-)$ lies in $[\mathcal{A}, \mathcal{B}]^{\prime} ;$ for we have the analogue of $(2.24)$ with $[\mathcal{A}, \mathcal{B}]^{\prime}$ replacing $[\mathcal{A}, \mathcal{B}]$.

The 2-natural isomorphism (2.20) exhibits $[\mathcal{A}, \mathcal{B}]$, when it exists, as a representing object for the 2 -functor $\mathcal{V}$-CAT $(-\otimes \mathcal{A}, \mathcal{B})$. It is conversely true that, if this 2 -functor is representable by some $\mathcal{D}$, then $[\mathcal{A}, \mathcal{B}]$ exists and is isomorphic to $\mathcal{D}$ - at least if $\mathcal{V}_{0}$ has an initial object 0 . For taking $\mathcal{C}$ to be $\mathcal{I}$ in $(2.20)$, with $[\mathcal{A}, \mathcal{B}]$ replaced by $\mathcal{D}$, we identify the objects of $\mathcal{D}$ with the $\mathcal{V}$-functors $\mathcal{A} \longrightarrow \mathcal{B}$; while taking $\mathcal{C}$ to be the $\mathcal{V}$-category with two objects 0 and 1 , with $\mathcal{C}(0,0)=\mathcal{C}(1,1)=I$, with $\mathcal{C}(1,0)=0$, and with $\mathcal{C}(0,1)$ an arbitrary object $X$ of $\mathcal{V}$, we easily see that $\mathcal{D}(T, S)=\int_{A} \mathcal{B}(T A, S A)$.

It follows from 1.10 that, so far as it exists, $[\mathcal{A}, \mathcal{B}]$ is uniquely 2 -functorial in $\mathcal{A}$ and $\mathcal{B}$ in such a way that (2.20) is 2-natural in every variable; which by 1.9 is equivalent to the 2-naturality in each variable of $E:[\mathcal{A}, \mathcal{B}] \otimes \mathcal{A} \longrightarrow \mathcal{B}$. Using this latter criterion we easily see that, for $M: \mathcal{A}^{\prime} \longrightarrow \mathcal{A}$ and $N: \mathcal{B} \longrightarrow \mathcal{B}^{\prime}$, the $\mathcal{V}$-functor $[M, N]:[\mathcal{A}, \mathcal{B}] \longrightarrow\left[\mathcal{A}^{\prime}, \mathcal{B}^{\prime}\right]$ sends $T$ to $N T M$ and is determined on hom-objects by

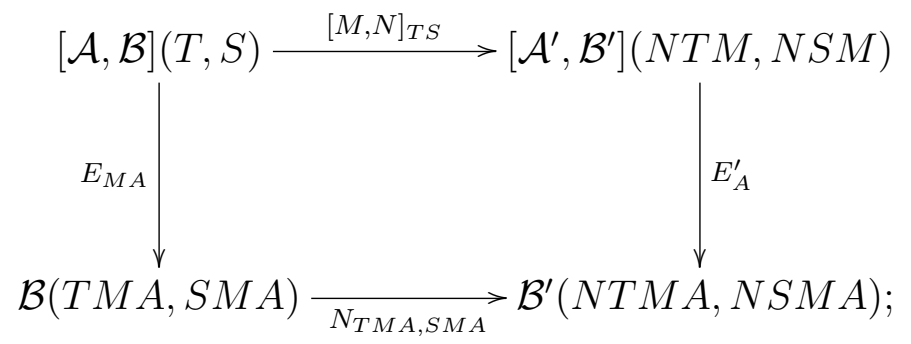


while the $\mathcal{V}$-natural transformation $[\mu, \nu]:[M, N] \longrightarrow\left[M^{\prime}, N^{\prime}\right]$ is given by

$$
[\mu, \nu]_{T}=\nu T \mu: N T M \longrightarrow N^{\prime} T M^{\prime} .
$$

As for the interplay of functor categories with duality, we clearly have a 2-natural isomorphism

$$
\left[\mathcal{A}^{\mathrm{op}}, \mathcal{B}^{\mathrm{op}}\right] \cong[\mathcal{A}, \mathcal{B}]^{\mathrm{op}}
$$

either side existing if the other does.

The 2 -functor $-\otimes \mathcal{A}: \mathcal{V}$-CAT $\longrightarrow \mathcal{V}$-CAT has a right adjoint $[\mathcal{A},-]$ when $[\mathcal{A}, \mathcal{B}]$ exists for all $\mathcal{B}$; and by the above, only then, if $\mathcal{V}_{0}$ has an initial object. This right adjoint surely exists when $\mathcal{A}$ is small, in the extended sense of 2.1 ; and for many $\mathcal{V}$ it exists only then. For instance, a result of Freyd (see [73]) ${ }^{1}$ shows that, when $\mathcal{V}=\operatorname{Set}, \mathcal{A}$ is necessarily small if $[\mathcal{A}, \mathcal{V}]$ exists. Yet when $\mathcal{V}_{0}$ is an ordered set, as in the cases $\mathcal{V}=2$ and $\mathcal{V}=\overline{\mathbb{R}}_{+}$, $[\mathcal{A}, \mathcal{B}]$ exists for every $\mathcal{A}$ and $\mathcal{B}$.

In this sense the symmetric monoidal 2-category $\mathcal{V}$-CAT is "partially closed"; while the symmetric monoidal 2 -category $\mathcal{V}$-Cat of small $\mathcal{V}$-categories is actually closed.

The partial closedness is sufficient to imply the isomorphisms (1.26) and (1.27) of 1.5, which here read:

$$
[\mathcal{I}, \mathcal{B}] \cong \mathcal{B}, \quad[\mathcal{C} \otimes \mathcal{A}, \mathcal{B}] \cong[\mathcal{C},[\mathcal{A}, \mathcal{B}]]
$$

the latter in the sense that either side exists if the other does, provided $[\mathcal{A}, \mathcal{B}]$ exists which follows alternatively from the Fubini Theorem (2.8).

\subsection{The (strong) Yoneda lemma for $\mathcal{V}$-CAT; the Yoneda embedding $\mathcal{A} \longrightarrow\left[\mathcal{A}^{\mathrm{op}}, \mathcal{V}\right]$}

We can now give a stronger version of the Yoneda Lemma of 1.9, no longer expressed by a mere bijection of sets, but by a Yoneda isomorphism in $\mathcal{V}_{0}$.

Given a $\mathcal{V}$-functor $F: \mathcal{A} \longrightarrow \mathcal{V}$ and an object $K$ of $\mathcal{A}$ as in 1.9, we have the map $F_{K A}: \mathcal{A}(K, A) \longrightarrow[F K, F A]$, which is $\mathcal{V}$-natural in $A$ by $1.8(\mathrm{~b})$. The transform

$$
\phi_{A}: F K \longrightarrow[\mathcal{A}(K, A), F A]
$$

of $F_{K A}$ under the adjunction $\mathcal{V}_{0}(X,[Y, Z]) \cong \mathcal{V}_{0}(Y,[X, Z])$ is $\mathcal{V}$-natural in $A$ by $1.8(\mathrm{~m})$. The stronger Yoneda Lemma is the assertion that (2.30) expresses $F K$ as the end $\int_{A}[\mathcal{A}(K, A), F A]$, so that we have an isomorphism

$$
\phi: F K \cong[\mathcal{A}, \mathcal{V}](\mathcal{A}(K,-), F] .
$$

For the proof, consider any $\mathcal{V}$-natural $\alpha_{A}: X \longrightarrow[\mathcal{A}(K, A), F A]$; its transform $\bar{\alpha}_{A}: \mathcal{A}(K, A) \longrightarrow[X, F A]$ under the adjunction, being $\mathcal{V}$-natural in $A$ by $1.8(\mathrm{~m})$, is the composite

$$
\mathcal{A}(K, A) \underset{F_{K A}}{\longrightarrow}[F K, F A] \underset{[\eta, 1]}{\longrightarrow}[X, F A]
$$

\footnotetext{
${ }^{1}$ For a more useful citation, see Peter Freyd and Ross Street, On the size of categories, Theory and Applications of Categories 1 (1995) 174-178.

http://www.tac.mta.ca/tac/volumes/1995/n9/1-09abs.html
} 
for a unique $\eta: X \longrightarrow F K$, by the Yoneda Lemma of 1.9 in the form (1.48). This is equivalent to the assertion that $\alpha_{A}=\phi_{A} \eta$ for a unique $\eta$; which completes the proof.

The image under $V=\mathcal{V}_{0}(I,-): \mathcal{V}_{0} \longrightarrow$ Set of $(2.31)$ is a bijection

$$
\mathcal{V}_{0}(I, F K) \longrightarrow[\mathcal{A}, \mathcal{V}]_{0}(\mathcal{A}(K,-), F)
$$

This sends $\eta: I \longrightarrow F K$ to $\phi \eta: \mathcal{A}(K,-) \longrightarrow F$, whose component $\mathcal{A}(K, A) \longrightarrow F A$ is $E_{A} \phi \eta$ by (2.12), or $\phi_{A} \eta$. By the definition (2.30) of $\phi_{A}$, this is at once seen to be the composite (1.47). Hence the weak Yoneda Lemma of 1.9, which we have used to prove the present strong Yoneda Lemma, is subsumed under the latter as its underlying bijection.

The special case $F=\mathcal{A}(L,-): \mathcal{A} \longrightarrow \mathcal{V}$ of the present Yoneda Lemma strengthens the last assertion of 1.9 to

$$
\mathcal{A}(L, K) \cong[\mathcal{A}, \mathcal{V}](\mathcal{A}(K,-), \mathcal{A}(L,-)) .
$$

Since (2.31) is true without any smallness restriction on $\mathcal{A}$, its $\mathcal{V}$-naturality must in general be discussed (cf. 2.2) in terms of the $\mathcal{V}$-functor $\operatorname{Hom}_{\mathcal{A}}: \mathcal{A}^{\mathrm{op}} \otimes \mathcal{A} \longrightarrow \mathcal{V}$ and some $\mathcal{V}$-functor $P: \mathcal{C} \otimes \mathcal{A} \longrightarrow \mathcal{V}$. We assert that

$$
\phi=\phi_{C K}: P(C, K) \cong[\mathcal{A}, \mathcal{V}](\mathcal{A}(K,-), P(C,-))
$$

is $\mathcal{V}$-natural in $C$ and $K$, when the right side is made $\mathcal{V}$-functorial as in (2.18). This follows from (2.19); for $E_{A} \phi=\phi_{A}: P(C, K) \rightarrow[\mathcal{A}(K, A), P(C, A)]$ is $\mathcal{V}$-natural in $C$ and $K$ by $1.8(\mathrm{~m})$, since $\mathcal{A}(K, A) \longrightarrow[P(C, K), P(C, A)]$ is so by $1.8(\mathrm{~b})$ and $1.8(\mathrm{c})$.

When $[\mathcal{A}, \mathcal{V}]$ exists, $\operatorname{Hom}_{\mathcal{A}}: \mathcal{A}^{\mathrm{op}} \otimes \mathcal{A} \longrightarrow \mathcal{V}$ corresponds under $(2.20)$ to a $\mathcal{V}$-functor

$$
Y: \mathcal{A}^{\mathrm{op}} \longrightarrow[\mathcal{A}, \mathcal{V}]
$$

sending $K$ to

$$
Y K=\mathcal{A}(K,-)
$$

being fully faithful by (2.32), $Y$ is called the Yoneda embedding. In these circumstances the $\mathcal{V}$-naturality $(2.33)$ can be more globally expressed by taking $[\mathcal{A}, \mathcal{V}]$ for $\mathcal{C}$ and $E:[\mathcal{A}, \mathcal{V}] \otimes$ $\mathcal{A} \longrightarrow \mathcal{V}$ for $P$; the right side of (2.33) is then by (2.24) the value of the $\mathcal{V}$-functor

$$
\mathcal{A} \otimes[\mathcal{A}, \mathcal{V}] \underset{Y^{\mathrm{op}} \otimes 1}{\longrightarrow}[\mathcal{A}, \mathcal{V}]^{\mathrm{op}} \otimes[\mathcal{A}, \mathcal{V}] \underset{\operatorname{Hom}_{[\mathcal{A}, \mathcal{V}]}}{\longrightarrow} \mathcal{V}
$$

so that, with respect to this functoriality,

$$
\text { the Yoneda isomorphism (2.31) is } \mathcal{V} \text {-natural in } K \text { and } F \text {. }
$$

The extra-variable result of 1.9 also has a lifting to the present context. Let $F: \mathcal{B}^{\text {op }} \otimes \mathcal{A} \longrightarrow \mathcal{V}$ and $K: \mathcal{B} \longrightarrow \mathcal{A}$. By (2.33) we have an isomorphism $F(C, K B) \cong$ $[\mathcal{A}, \mathcal{V}](\mathcal{A}(K B,-), F(C,-)), \mathcal{V}$-natural in $C$ and $B$. The Fubini Theorem (2.8) now gives

$$
\int_{B} F(B, K B) \cong\left[\mathcal{B}^{\mathrm{op}} \otimes \mathcal{A}, \mathcal{V}\right](\mathcal{A}(K ?,-), F(?,-)),
$$

either side existing if the other does. 


\subsection{The free $\mathcal{V}$-category on a Set-category}

We henceforth suppose, in addition to all our former assumptions on $\mathcal{V}$, that the underlying category $\mathcal{V}_{0}$ is cocomplete.

Then the ordinary functor $V=\mathcal{V}_{0}(I,-): \mathcal{V}_{0} \longrightarrow$ Set has a left adjoint $(-) \cdot I$, sending the set $E$ to the coproduct $E \cdot I$ of $E$ copies of $I$ in $\mathcal{V}_{0}$. This functor sends the cartesian product in Set to the tensor product in $\mathcal{V}_{0}$, in that we clearly have

$$
(E \times F) \cdot I \cong(E \cdot I) \otimes(F \cdot I), \quad 1 \cdot I \cong I
$$

The 2-functor $(-)_{0}: \mathcal{V}$-CAT $\longrightarrow$ CAT now has a left adjoint $(-)_{\mathcal{V}}$. The free $\mathcal{V}$-category $\mathcal{L}_{\mathcal{V}}$ on the ordinary locally-small category $\mathcal{L}$ has the same objects as $\mathcal{L}$, and has hom-objects $\mathcal{L}_{\mathcal{V}}(K, L)=\mathcal{L}(K, L) \cdot \mathcal{I}$; its composition law and its identities are induced from those of $\mathcal{L}$ using the isomorphisms (2.39).

For the proof, we have first to verify that $\mathcal{L}_{\mathcal{V}}$, as defined, satisfies the $\mathcal{V}$-category axioms (1.3) and (1.4); this is trivial from the corresponding axioms for $\mathcal{L}$.

There is an evident functor $\psi: \mathcal{L} \longrightarrow\left(\mathcal{L}_{\mathcal{V}}\right)_{0}$ which is the identity on objects, and which is defined on morphisms by the obvious map $\mathcal{L}(K, L) \longrightarrow \mathcal{V}_{0}(I, \mathcal{L}(K, L) \cdot I)$. This $\psi$ is the unit of the 2-adjunction in question.

In fact it is easy to see that, for a $\mathcal{V}$-category $\mathcal{B}$ and a functor $T: \mathcal{L} \longrightarrow \mathcal{B}_{0}$, there is exactly one $\mathcal{V}$-functor $\bar{T}: \mathcal{L}_{\mathcal{V}} \longrightarrow \mathcal{B}$ with $\bar{T}_{0} \psi=T$ : on objects we have $\bar{T} K=T K$, while $\bar{T}_{K L}: \mathcal{L}(K, L) \cdot I \longrightarrow \mathcal{B}(T K, T L)$ is the transform of $T_{K L}: \mathcal{L}(K, L) \longrightarrow \mathcal{B}_{0}(T K, T L)$ under the adjunction $(-) \cdot I \dashv V$. Verification of (1.5) and (1.6) for $\bar{T}$ is easy.

Finally, if $\alpha: T \longrightarrow S: \mathcal{L} \longrightarrow \mathcal{B}_{0}$, there is a unique $\mathcal{V}$-natural $\bar{\alpha}: \bar{T} \longrightarrow \bar{S}: \mathcal{L}_{\mathcal{V}} \longrightarrow \mathcal{B}$ with $\bar{\alpha}_{0} \psi=\alpha$; the component $\bar{\alpha}_{K}$ must be $\alpha_{K} \in \mathcal{B}_{0}(T K, S K)$, and again verification of (1.7) for $\bar{\alpha}$ is easy. This completes the proof.

If $\mathcal{L}$ is a small category, $\mathcal{L}_{\mathcal{V}}$ is clearly a small $\mathcal{V}$-category, and we can form the functor category $\left[\mathcal{L}_{\mathcal{V}}, \mathcal{B}\right]$. Since the underlying ordinary category $\left[\mathcal{L}_{\mathcal{V}}, \mathcal{B}\right]_{0}$ of this is $\mathcal{V}$ - $\operatorname{CAT}\left(\mathcal{L}_{\mathcal{V}}, \mathcal{B}\right)$ by $(2.16)$, the 2 -adjunction above gives

$$
\left[\mathcal{L}_{\mathcal{V}}, \mathcal{B}\right]_{0} \cong\left[\mathcal{L}, \mathcal{B}_{0}\right]
$$

\subsection{Universe-enlargement $\mathcal{V} \subset \mathcal{V}^{\prime}$ in concrete cases; $[\mathcal{A}, \mathcal{B}]$ as a $\mathcal{V}^{\prime}$-category for large $\mathcal{A}$}

The possible non-existence of a $\mathcal{V}$-functor category $[\mathcal{A}, \mathcal{B}]$ when $\mathcal{A}$ is large (= non-small) is somewhat tedious, forcing us into such circumlocutions as using (2.18) instead of (2.24), or (2.33) instead of (2.37) - which then propagate themselves throughout the applications below.

Yet when $\mathcal{V}=$ Set, we always do have $[\mathcal{A}, \mathcal{B}]$ as a category; albeit not in general a Set-category (= locally-small category) when $\mathcal{A}$ and $\mathcal{B}$ are Set-categories, unless $\mathcal{A}$ is small. It can however be seen as a Set $^{\prime}$-category, where $\mathbf{S e t}^{\prime}$ is the category of sets in some larger universe containing ob $\mathcal{A}$ as an element - we are taking the view that ob $\mathcal{A}$ is always an honest set, and supposing that every set belongs to some universe. 
We may ask whether such a view can be imitated for a general $\mathcal{V}$. Consider first the various examples of a symmetric monoidal closed $\mathcal{V}$ given in 1.1. These are of two types: either $\mathcal{V}_{0}$ is a particular ordered set, such as 2 or $\overline{\mathbb{R}}_{+}$, in which case $[\mathcal{A}, \mathcal{B}]$ exists for all $\mathcal{A}$; or $\mathcal{V}_{0}$ consists of the small models of some theory - small sets, small categories, small abelian groups, small differential graded $R$-modules, and so on. In the latter case we can clearly consider the category $\mathcal{V}_{0}^{\prime}$ of models in a larger $\mathbf{S e t}^{\prime}$, which gives a symmetric monoidal closed category $\mathcal{V}^{\prime}$, in which $\mathcal{V}$ with its symmetric monoidal closed structure is faithfully embedded. Moreover $\mathcal{V}_{0}^{\prime}$ admits all limits and colimits which are small by reference to Set'; and the inclusion $\mathcal{V}_{0} \longrightarrow \mathcal{V}_{0}^{\prime}$ preserves all limits and colimits that exist in $\mathcal{V}_{0}$. (We are supposing that our original universe contains infinite sets; otherwise we do not have the colimit-preservation above.)

Then, for $\mathcal{V}$-categories $\mathcal{A}$ and $\mathcal{B}$, we always have a $\mathcal{V}^{\prime}$-category $[\mathcal{A}, \mathcal{B}]$, where Set $^{\prime}$ is taken large enough to include ob $\mathcal{A}$. Since $\mathcal{V}_{0} \longrightarrow \mathcal{V}_{0}^{\prime}$ preserves limits, to say that $[\mathcal{A}, \mathcal{B}](T, S)$ exists in the sense of 2.2 is precisely to say that the object $[\mathcal{A}, \mathcal{B}](T, S)$ of $\mathcal{V}^{\prime}$ actually lies in $\mathcal{V}$; and to say that $[\mathcal{A}, \mathcal{B}]$ exists in the sense of $2.2-$ that is, as a $\mathcal{V}$-category - is to say that $[\mathcal{A}, \mathcal{B}](T, S) \in \mathcal{V}$ for all $T, S$.

That a similar thing is possible for a perfectly general $\mathcal{V}$ follows from Day [17]. Using his construction, one can show that there is always a suitable $\mathcal{V}^{\prime}$ whose underlying ordinary category $\mathcal{V}_{0}^{\prime}$ is a full reflective subcategory of $\left[\mathcal{V}_{0}^{\text {op }}\right.$, Set $\left.{ }^{\prime}\right]$, into which $\mathcal{V}_{0}$ is embedded by Yoneda. Since the monoidal structure of $\mathcal{V}^{\prime}$ is defined by coends (relative to Set'), we defer a description of it until 3.11 and 3.12 below, after these have been introduced. 


\section{Chapter 3}

\section{Indexed limits and colimits}

\subsection{Indexing types; limits and colimits; Yoneda isomorphisms}

For a discussion of completeness, or of Kan extensions, or of density, in the context of $\mathcal{V}$-categories, ordinary limits - defined in terms of the representability of cones - do not suffice; we need the wider notion of indexed limit. These latter fully deserve the name of "limit", since they enjoy all the formal properties expected of a limit-notion, and include the classical cone-type limits as a special case. When $\mathcal{V}=\mathbf{S e t}$, in contrast to the situation for a general $\mathcal{V}$, an indexed limit may be expressed as an ordinary limit; yet even here, the indexed limit is a valuable concept in its own right - its meaning is scarcely clarified by its construction as an ordinary limit. Since the cone-type limits have no special position of dominance in the general case, we go so far as to call indexed limits simply "limits", where confusion seems unlikely.

We may think of a $\mathcal{V}$-functor $F: \mathcal{K} \longrightarrow \mathcal{V}$ as an indexing type - a small one when $\mathcal{K}$ is small, which will be the usual case, but not the only one. Then we may think of a $\mathcal{V}$-functor $G: \mathcal{K} \longrightarrow \mathcal{B}$ as a diagram in $\mathcal{B}$ of type $F$. For each $B \in \mathcal{B}$, we get from $G$ a $\mathcal{V}$-functor $\mathcal{B}(B, G-): \mathcal{K} \longrightarrow \mathcal{V}$; and we can consider the existence in $\mathcal{V}$ of the object $[\mathcal{K}, \mathcal{V}](F, \mathcal{B}(B, G-))$. If this exists for all $B$ - as it surely does when $\mathcal{K}$ is small - it is as in $(2.18)$ the value of a $\mathcal{V}$-functor $\mathcal{B}^{\text {op }} \longrightarrow \mathcal{V}$. If this $\mathcal{V}$-functor not only exists but admits a representation

$$
\mathcal{B}(B,\{F, G\}) \cong[\mathcal{K}, \mathcal{V}](F, \mathcal{B}(B, G-)),
$$

with counit say

$$
\mu: F \rightarrow \mathcal{B}(\{F, G\}, G-),
$$

we call the representation $(\{F, G\}, \mu)$ the limit of $G$ indexed by $F$. An evidently equivalent formulation not mentioning $[\mathcal{K}, \mathcal{V}]$ explicitly is the following: each component $\mu_{K}$ of $\mu$ induces by Yoneda a family

$$
\mathcal{B}(B,\{F, G\}) \longrightarrow[F K, \mathcal{B}(B, G K)]
$$

$\mathcal{V}$-natural in $B$ and $K$; and $(\{F, G\}, \mu)$ is the limit precisely when (3.3) is an end over $K$ for each $B$. In practice, by the usual abuse of notation, we call the representing object $\{F, G\}$ the limit, consigning to the background the counit $\mu$-or equivalently the choice of the $\mathcal{V}$-natural isomorphism (3.1). 
If we take the view of 2.6 , that $[\mathcal{K}, \mathcal{V}]$ always exists, if not as a $\mathcal{V}$-category, then as a $\mathcal{V}^{\prime}$-category for some larger $\mathcal{V}^{\prime}$, we need not be concerned with the existence of $[\mathcal{K}, \mathcal{V}](F, \mathcal{B}(B, G-))$, but only with its representability in the form (3.1) for some $\{F, G\} \in \mathcal{B}$. If it is so representable, the right side of (3.1) in fact lies in $\mathcal{V}$, since the left side does. We shall in future take this view whenever it is convenient - usually without bothering to change " $\mathcal{V}$-functor" to " $\mathcal{V}$ '-functor", and so on.

Applying $V$ to the isomorphism (3.1) gives a bijection of sets

$$
\mathcal{B}_{0}(B,\{F, G\}) \cong\left[\mathcal{K}, \mathcal{V}_{0}\right](F, \mathcal{B}(B, G-))
$$

but the requirement that $(\{F, G\}, \mu)$ induce a bijection (3.4) is, except in the special case when $V$ is conservative, strictly weaker than the requirement that it induce an isomorphism (3.1), and does not suffice to make $(\{F, G\}, \mu)$ the limit; see 3.7 below. However it does suffice to detect $(\{F, G\}, \mu)$ as the limit if the limit is known to exist; for the right side of (3.4) admits at most one representation to within isomorphism. An element $\alpha: F \longrightarrow \mathcal{B}(B, G-)$ of the right side of (3.4) may be called an $(F, B)$-cylinder over $G$; then the bijection (3.4) is the assertion that every such cylinder factorizes through the counit-cylinder $\mu$ as $\alpha=\mathcal{B}(f, 1) \mu$ for a unique $f: B \longrightarrow\{F, G\}$.

An indexed colimit in $\mathcal{B}$ is nothing but an indexed limit in $\mathcal{B}^{\text {op }}$; but it is usual to replace $\mathcal{K}$ by $\mathcal{K}^{\text {op }}$ in the definition, so that we still have $G: \mathcal{K} \longrightarrow \mathcal{B}$, but now have $F: \mathcal{K}^{\text {op }} \longrightarrow \mathcal{V}$. Then the colimit of $G$ indexed by $F$ is the representing object $F \star G$ in

$$
\mathcal{B}(F \star G, B) \cong\left[\mathcal{K}^{\mathrm{op}}, \mathcal{V}\right](F, \mathcal{B}(G-, B))
$$

with unit

$$
\nu: F \longrightarrow \mathcal{B}(G-, F \star G) .
$$

When $\mathcal{B}=\mathcal{V}$, we have from (2.13) and (3.1)

$$
\{F, G\} \cong[\mathcal{K}, \mathcal{V}](F, G) \quad \text { for } F, G: \mathcal{K} \longrightarrow \mathcal{V}
$$

either side existing if the other does. Hence (3.1) and (3.5) can be written

$$
\mathcal{B}(B,\{F, G\}) \cong\{F, \mathcal{B}(B, G-)\}, \quad \mathcal{B}(F \star G, B) \cong\{F, \mathcal{B}(G-, B)\} .
$$

Thus, just as classical limits and colimits in an ordinary category $\mathcal{B}$ are defined, via representability, in terms of the primitive limits - sets of cones - in Set, so indexed limits and colimits in a $\mathcal{V}$-category $\mathcal{B}$ are defined, via representability, in terms of indexed limits in $\mathcal{V}$; and thus ultimately, by 2.1 and 2.2 , in terms of ordinary limits in $\mathcal{V}_{0}$

Again, in the special case $\mathcal{B}=\mathcal{V}$, the $\mathcal{V}$-natural isomorphism $[F K,[G L, B]] \cong$ $[G L,[F K, B]]$ gives by $(3.5)$

$$
F \star G \cong G \star F \quad \text { for } F: \mathcal{K}^{\mathrm{op}} \longrightarrow \mathcal{V} \text { and } G: \mathcal{K} \longrightarrow \mathcal{V} .
$$

The $\mathcal{V}$-natural Yoneda isomorphism (2.33) immediately gives the values of $\{F, G\}$ and $F \star G$ when $F$ is representable, for any $G: \mathcal{K} \longrightarrow \mathcal{B}$; namely

$$
\{\mathcal{K}(K,-), G\} \cong G K, \quad \mathcal{K}(-, K) \star G \cong G K .
$$


More precisely, the cylinder $G_{K-}: \mathcal{K}(K,-) \longrightarrow \mathcal{B}(G K, G-)$ as counit exhibits $G K$ as $\{\mathcal{K}(K,-), G\}$. We refer to (3.10) as Yoneda isomorphisms; by (3.7), the first of them includes as a special case the original Yoneda isomorphism $(2.31)$.

When $\mathcal{K}$ is small, the maps $(F, G) \mapsto\{F, G\}$ and $(F, G) \mapsto F \star G$ are by 1.10 the object-functions of $\mathcal{V}$-functors

$$
\{,\}:\left([\mathcal{K}, \mathcal{V}]^{\mathrm{op}} \otimes[\mathcal{K}, \mathcal{B}]\right)^{\prime} \longrightarrow \mathcal{B}, \quad \star:\left(\left[\mathcal{K}^{\mathrm{op}}, \mathcal{V}\right] \otimes[\mathcal{K}, \mathcal{B}]\right)^{\prime} \longrightarrow \mathcal{B}
$$

where the domain consists of those pairs $(F, G)$ for which $\{F, G\}$ or $F \star G$ exists; and in such a way that $(3.1)$ and $(3.5)$ are $\mathcal{V}$-natural in $(F, G)$. Then $(2.37)$ gives:

The Yoneda isomorphisms (3.10) are $\mathcal{V}$-natural in $K$ and $G$.

\subsection{Preservation of limits and colimits}

We call the limit $\{F, G\}$ a small limit if the indexing-type $F: \mathcal{K} \longrightarrow \mathcal{V}$ is small; that is, if $\mathcal{K}$ is small. We call the $\mathcal{V}$-category $\mathcal{B}$ complete if it admits all small limits; and dually, cocomplete if it admits all small colimits. By $(3.7)$ and $2.2, \mathcal{V}$ is complete; we shall see in 3.10 below that $\mathcal{V}$ is also cocomplete.

A $\mathcal{V}$-functor $T: \mathcal{B} \longrightarrow \mathcal{C}$ is said to preserve the limit $\{F, G\}$ if the composite cylinder

$$
F \underset{\mu}{\longrightarrow} \mathcal{B}(\{F, G\}, G-) \underset{T}{\longrightarrow} \mathcal{C}(T\{F, G\}, T G-)
$$

where $\mu$ is the counit of $\{F, G\}$, exhibits $T\{F, G\}$ as $\{F, T G\}$; in other words, if $\{F, T G\}$ exists and if the evident canonical map

$$
T\{F, G\} \longrightarrow\{F, T G\}
$$

is an isomorphism.

We call $T: \mathcal{B} \longrightarrow \mathcal{C}$ continuous (the dual is cocontinuous) if it preserves all small limits that exist; of course this condition is of interest particularly when $\mathcal{B}$ is complete.

The representables $\mathcal{B}(B,-): \mathcal{B} \longrightarrow \mathcal{V}$ are more than continuous: by (3.8) they preserve every limit that exists; while in their totality, by definition, they "detect" the existence of $\{F, G\}$.

For a very general result on the preservation of limits, consider a functor $Q: \mathcal{C}^{\text {op }} \otimes$ $\mathcal{B} \longrightarrow \mathcal{V}$, and let $\mathcal{B}^{\prime} \subset \mathcal{B}$ be the full subcategory of those $B$ for which $Q(-, B)$ is representable, say as $Q(C, B) \cong \mathcal{C}(C, T B)$; we shall refer to the objects of $\mathcal{B}^{\prime}$ as "those $B$ for which $T B$ exists". Let $\{F, G\}$ be a limit in $\mathcal{B}$ which is preserved by $Q(C,-): \mathcal{B} \longrightarrow \mathcal{V}$ for each $C$. Finally, let $T G$ exist; that is, suppose that $G$ takes its values in $\mathcal{B}^{\prime}$. Then we clearly have

$$
T\{F, G\} \cong\{F, T G\}
$$

either side existing if the other does; for, using (3.7), the two sides of (3.15) represent the isomorphic $\mathcal{V}$-functors $Q(C,\{F, G\})$ and $[\mathcal{K}, \mathcal{V}](F, \mathcal{C}(C, T G)) \cong[\mathcal{K}, \mathcal{V}](F, Q(C, G-)) \cong$ $\{F, Q(C, G-)\}$. 
If $Q$ here is defined by $Q(C, B)=\mathcal{B}(S C, B)$ where $S: \mathcal{C} \longrightarrow \mathcal{B}$, then $T: \mathcal{B}^{\prime} \longrightarrow \mathcal{C}$ is the partial right adjoint of $S$. Since $\mathcal{B}(S C,-)$ preserves all limits, we have (3.15) whenever $\{F, G\}, T\{F, G\}$, and $T G$ exist. This conclusion may be expressed as: partial right adjoints in so far as they are defined, preserve limits.

In the special case where $S$ in fact has a right adjoint $T: \mathcal{B} \longrightarrow \mathcal{C}$, the conclusion becomes: right adjoints preserve all limits that exist.

\subsection{Limits in functor categories; double limits and iterated limits}

We now consider limits in a functor category $[\mathcal{A}, \mathcal{B}]$. Let $G: \mathcal{K} \longrightarrow[\mathcal{A}, \mathcal{B}]$ correspond under the adjunction $(2.20)$ to $P: \mathcal{K} \otimes \mathcal{A} \longrightarrow \mathcal{B}$. In order to avoid formulae containing various letters whose inter-relation must be explained in a subordinate clause, let us write $(2.22)$ with cyphers for variables, in the form $P(-, ?)=(G-)$ ?; so that, in particular, $(G-) A$ denotes $P(-, A): \mathcal{K} \longrightarrow \mathcal{B}$. Let $F: \mathcal{K} \longrightarrow \mathcal{V}$.

Then, if $\{F,(G-) A\}$ exists for each $A$, the limit $\{F, G\}$ in $[\mathcal{A}, \mathcal{B}]$ exists, and we have

$$
\{F, G\} A=\{F,(G-) A\} .
$$

For, if we define $\{F, G\}$ to be the $\mathcal{V}$-functor whose value at $A$ is given by (3.16), we have

$$
\begin{aligned}
{[\mathcal{A}, \mathcal{B}](H,\{F, G\}) } & =\int_{A} \mathcal{B}(H A,\{F,(G-) A\}) & & \text { by }(3.16) \\
& \cong \int_{A}[\mathcal{K}, \mathcal{V}](F, \mathcal{B}(H A,(G-) A)) & & \text { by }(3.1) \\
& \cong[\mathcal{K}, \mathcal{V}]\left(F, \int_{A} \mathcal{B}(H A,(G-) A)\right) & & \text { by }(2.3) \\
& =[\mathcal{K}, \mathcal{V}](F,[\mathcal{A}, \mathcal{B}](H, G-)) & & \text { by }(2.10) .
\end{aligned}
$$

In these circumstances we say that the limit $\{F, G\}$ in $[\mathcal{A}, \mathcal{B}]$ exists pointwise; it comes to the same thing to say that $\{F, G\}$ exists and is preserved by each $E_{A}:[\mathcal{A}, \mathcal{B}] \longrightarrow \mathcal{B}$. Clearly if $\mathcal{B}$ is complete [respectively cocomplete] so is $[\mathcal{A}, \mathcal{B}]$, all small limits [respectively colimits] existing pointwise in $[\mathcal{A}, \mathcal{B}]$.

When $\mathcal{B}$ is not complete, fortuitous non-pointwise limits may exist in $[\mathcal{A}, \mathcal{B}]$, even when $\mathcal{V}=$ Set. Take $\mathcal{A}=\mathbf{2}$ and let $\mathcal{B}$ be the category generated by the graph

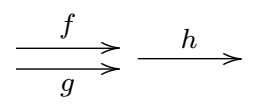

with the relation $h f=h g$. Then the map $(f, h): g \longrightarrow h$ given by

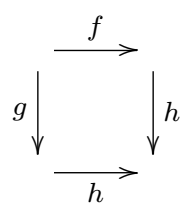

in $[2, \mathcal{B}]$ is monomorphic, although $h$ is not monomorphic in $\mathcal{B}$. Since $x$ is monomorphic in any category precisely when

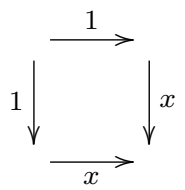


is a pullback, the pullback of $(f, h)$ by itself in $[2, \mathcal{B}]$ is a non-pointwise limit. The existence of a non-pointwise limit in $[\mathcal{A}, \mathcal{B}]$ says something about $[\mathcal{A}, \mathcal{B}]$ as a category, ignoring its relation to $\mathcal{A}$ and $\mathcal{B}$; while the existence of a pointwise limit says something about $[\mathcal{A}, \mathcal{B}]$ seen precisely as the functor category, and is really a completeness assertion about $\mathcal{B}$.

Applying the above, in the colimit case, to $[\mathcal{K}, \mathcal{V}]$ where $\mathcal{K}$ is small, we get from (3.9) and (3.10) a pointwise colimit

$$
F \star Y \cong F
$$

for any $F: \mathcal{K} \longrightarrow \mathcal{V}, Y$ being the Yoneda embedding $\mathcal{K}^{\mathrm{op}} \longrightarrow[\mathcal{K}, \mathcal{V}]$ of $(2.34)$. This gives a canonical representation of any $F: \mathcal{K} \longrightarrow \mathcal{V}$ as an (indexed) colimit of representables. In contrast, $Y$ preserves whatever limits exist in $\mathcal{K}^{\mathrm{op}}$, sending them to pointwise limits in $[\mathcal{K}, \mathcal{V}] ;$ for $E_{K} Y \cong \mathcal{K}(-, K): \mathcal{K}^{\mathrm{op}} \longrightarrow \mathcal{V}$.

We can now give the general Fubini Theorem relating repeated limits to double limits. Suppose that $F: \mathcal{K} \longrightarrow \mathcal{V}$ and $G: \mathcal{K} \longrightarrow[\mathcal{A}, \mathcal{B}]$, the latter corresponding to $P: \mathcal{K} \otimes \mathcal{A} \longrightarrow \mathcal{B}$, are such that the limit $\{F, G\}$ exists pointwise; and let $H: \mathcal{A} \longrightarrow \mathcal{V}$. Writing $H \otimes F: \mathcal{K} \otimes \mathcal{A} \longrightarrow \mathcal{V}$ for the functor sending $(K, A)$ to $H A \otimes F K$, we have a canonical isomorphism

$$
\{H,\{F, G\}\} \cong\{H \otimes F, P\},
$$

either side existing if the other does. ${ }^{1}$

For by (3.1) the left side of (3.18) represents

$$
\begin{aligned}
{[\mathcal{A}, \mathcal{V}](H ?, \mathcal{B}(B,\{F, G\} ?)) } & =[\mathcal{A}, \mathcal{V}](H ?, \mathcal{B}(B,\{F-,(G-) ?\})) & & \text { by }(3.16) \\
& \cong[\mathcal{A}, \mathcal{V}](H ?,[\mathcal{K}, \mathcal{V}](F-, \mathcal{B}(B,(G-) ?))) & & \text { by }(3.1) \\
& =\int_{A}\left[H A, \int_{K}[F K, \mathcal{B}(B,(G K) A)]\right] & & \text { by }(2.10) \\
& \cong \int_{A} \int_{K}[H A,[F K, \mathcal{B}(B,(G K) A)]] & & \text { by }(2.3) \\
& \cong \int_{A} \int_{K}[H A \otimes F K, \mathcal{B}(B, P(K, A))] & & \text { by }(1.27) ;
\end{aligned}
$$

and by the Fubini Theorem (2.8) we can replace $\int_{A} \int_{K}$ here by $\int_{(A, K)}$, giving by $(2.10)$ and (3.1) the object represented by the right side of (3.18).

The use of the cyphers "?" and "-" here to keep track of unexpressed variables is presumably self-explanatory. If we extend it by allowing $\{F-, G-\}$ as an alternative notation for $\{F, G\}$, we can write (3.18), suppressing the letter $G$, as

$$
\{H ?,\{F-, P(-, ?)\}\} \cong\{H ? \otimes F-, P(-, ?)\} .
$$

Then there is no need for explicit mention of the functor category $[\mathcal{A}, \mathcal{B}]$, which may exist only as a $\mathcal{V}^{\prime}$-category in the sense of 2.6 ; nor for explicit mention of the pointwise existence of $\{F, G\}$, which is just the existence of $\{F-, P(-, A)\}$ for each value of $A$.

In this notation the general Fubini Theorem gives at once the general interchange of limits theorem: Let $F: \mathcal{K} \longrightarrow \mathcal{V}, H: \mathcal{A} \longrightarrow \mathcal{V}$, and $P: \mathcal{K} \otimes \mathcal{A} \longrightarrow \mathcal{B}$; and suppose that $\{F-, P(-, A)\}$ exists for each $A$ and that $\{H ?, P(K, ?)\}$ exists for each $K$. Then

$$
\{F-,\{H ?, P(-, ?)\}\} \cong\{H ?,\{F-, P(-, ?)\}\},
$$

\footnotetext{
${ }^{1}$ The statement of this theorem differs from the original text which has $F \otimes H$ instead of $H \otimes F$. However, the proof proves the statement in the form given here and the form given in the text would appear to contradict, say, (1.27).
} 
either side existing if the other does.

For the colimit form of (3.19) and (3.20), let $F: \mathcal{K}^{\mathrm{op}} \longrightarrow \mathcal{V}, H: \mathcal{A}^{\mathrm{op}} \longrightarrow \mathcal{V}$, and $P: \mathcal{K} \otimes$ $\mathcal{A} \longrightarrow \mathcal{B}$; then, under similar hypotheses,

$$
F-\star(H ? \star P(-, ?)) \cong(F \otimes H) \star P \cong H ? \star(F-\star P(-, ?)) .
$$

In fact (3.20) also follows alternatively from the general principle of (3.15); for the right side of (3.1) clearly preserves pointwise limits in the variable $G \in[\mathcal{K}, \mathcal{B}]$.

In the variable $F \in[\mathcal{K}, \mathcal{V}]$, the right side of (3.1) sends arbitrary colimits, by (3.8), to limits; of course all small colimits in $[\mathcal{K}, \mathcal{V}]$ are pointwise, since we have remarked that $\mathcal{V}$ will be shown to be cocomplete. Hence we get from the principle (3.15) the general theorem of the continuity of a limit in its index: Let $F: \mathcal{K}^{\mathrm{op}} \longrightarrow \mathcal{V}, G: \mathcal{K} \longrightarrow[\mathcal{A}, \mathcal{V}]$, and $T: \mathcal{A} \longrightarrow \mathcal{B}$; and suppose that $F \star G$ exists and that each $\{G K, T\}$ exists. Then

$$
\{F \star G, T\} \cong\{F,\{G-, T\}\}
$$

either side existing if the other does. The corresponding form for colimits, with $F$ and $T$ as above but now with $G: \mathcal{K} \longrightarrow\left[\mathcal{A}^{\text {op }}, \mathcal{V}\right]$, is

$$
(F \star G) \star T \cong F \star(G \star T) .
$$

In future, when we equate limits in a general theorem, the phrase "either side existing if the other does" is to be understood.

\subsection{The connexion with classical conical limits when $\mathcal{V}=$ Set}

We now consider, in the special case $\mathcal{V}=$ Set, the relation of indexed limits to the ordinary classical limits.

Writing $\Delta B: \mathcal{K} \longrightarrow \mathcal{B}$ for the constant functor at $B \in \mathcal{B}$, we have a natural bijection

$$
[\mathcal{K}, \mathcal{B}](\Delta B, G) \cong[\mathcal{K}, \operatorname{Set}](\Delta 1, \mathcal{B}(B, G-))
$$

between the set of (projective) cones over $G$ with vertex $\mathcal{B}$ and the set of cones over $\mathcal{B}(B, G-)$ with vertex the one-point set 1 . The ordinary, or classical, or conical limit [resp. colimit] of $G: \mathcal{K} \longrightarrow \mathcal{B}$ is the representing object $\lim G[\operatorname{resp}$. colim $G]$ in

$$
\mathcal{B}(B, \lim G) \cong[\mathcal{K}, \mathcal{B}](\Delta B, G), \quad \mathcal{B}(\operatorname{colim} G, B) \cong[\mathcal{K}, \mathcal{B}](G, \Delta B)
$$

existing when the right side here is representable. Using (3.24) we conclude from (3.1) and (3.5) that

$$
\lim G=\{\Delta 1, G\}, \quad \operatorname{colim} G=\Delta 1 \star G .
$$

Thus the classical limit of $G: \mathcal{K} \longrightarrow \mathcal{B}$, often called a limit indexed by $\mathcal{K}$, is in fact the limit of $G$ indexed by $\Delta 1: \mathcal{K} \longrightarrow$ Set. Of course, the counit (3.2) now corresponds to the limit-cone

$$
\mu_{K}: \lim G \longrightarrow G K
$$


When $\mathcal{B}=$ Set, taking $B=1$ in the first equation of (3.25) gives

$$
\lim G \cong[\mathcal{K}, \operatorname{Set}](\Delta 1, G) \text { for } G: \mathcal{K} \longrightarrow \text { Set }
$$

so that here the limit is the set of cones with vertex 1 . Then, as we said in 3.1, all conical limits and colimits are defined representably in terms of these primitive ones in Set; for (3.24) and (3.28) allow us to rewrite (3.25) in the form (analogous to (3.8))

$$
\mathcal{B}(B, \lim G) \cong \lim \mathcal{B}(B, G-), \quad \mathcal{B}(\operatorname{colim} G, B) \cong \lim \mathcal{B}(G-, B)
$$

Now consider an arbitrary $F: \mathcal{K} \longrightarrow$ Set. We have as in 1.10 the category el $F$ of elements of $F$, whose objects are pairs $(K, x)$ with $K \in \mathcal{K}$ and $x \in F K$, and whose maps $(K, x) \longrightarrow\left(K^{\prime}, x^{\prime}\right)$ are the $f: K \longrightarrow K^{\prime}$ in $\mathcal{K}$ for which $(F f) x=x^{\prime}$. Write $d:$ el $F \longrightarrow \mathcal{K}$ for the functor sending $(K, x)$ to $K$, and let $G: \mathcal{K} \longrightarrow$ Set be arbitrary. Then we have

$$
\lim (\operatorname{el} F \underset{d}{\longrightarrow} \mathcal{K} \underset{G}{\longrightarrow} \text { Set }) \cong[\mathcal{K}, \operatorname{Set}](F, G) .
$$

For an element of the left side is by (3.28) a cone $\alpha_{(K, x)}: 1 \longrightarrow G K$; to give such a cone is to give for each $x \in F K$ an element $\beta_{K} x=\alpha_{(K, x)}$ of $G K$; and the naturality of $\alpha$ as a cone translates exactly into the naturality of $\beta_{K}: F K \longrightarrow G K$.

Since a map of a representable $\mathcal{K}(K,-)$ into $F$ corresponds by Yoneda to an element $x \in F K$, we have a canonical inductive cone

$$
\mu_{(K, x)}: \mathcal{K}(K,-) \longrightarrow F
$$

in $[\mathcal{K}$, Set $]$ whose base is indexed by $(\mathrm{el} F)^{\mathrm{op}}$. The classical result on the expressibility of any $F: \mathcal{K} \longrightarrow$ Set as a canonical (conical) colimit of representables is the assertion that this is a colimit-cone; more precisely,

$$
F \cong \operatorname{colim}\left((\mathrm{el} F)^{\mathrm{op}} \underset{d^{\mathrm{op}}}{\longrightarrow} \mathcal{K}^{\mathrm{op}} \underset{Y}{\longrightarrow}[\mathcal{K}, \text { Set }]\right)
$$

with unit (3.31), where $Y$ is the Yoneda embedding. This follows from (3.30) and the characterization (3.29) of the colimit, since the left side of $(3.30)$ is also $\lim [\mathcal{K}, \operatorname{Set}]\left(Y d^{\text {op }}-, G\right)$ by Yoneda.

Because indexed limits are continuous in their index in the sense of (3.22), the colimitexpression $F \cong \operatorname{colim} \mathcal{K}(K,-)$ of (3.32) gives, for any $G: \mathcal{K} \longrightarrow \mathcal{B}$, a limit-expression $\{F, G\} \cong \lim \{\mathcal{K}(K,-), G\}$; so by the Yoneda isomorphism (3.10) we have

$$
\{F, G\} \cong \lim (\text { el } F \underset{d}{\longrightarrow} \mathcal{K} \underset{G}{\longrightarrow} \mathcal{B}),
$$

of which (3.30) is a special case by (3.7).

We conclude that, when $\mathcal{V}=$ Set, the classical conical limits are special cases of indexed limits by (3.26), while the general indexed limit can be expressed as a conical limit by (3.33). Since el $F$ is small when $\mathcal{K}$ is small, a Set-category $\mathcal{B}$ is complete in our sense of 3.2 above exactly when it admits all small conical limits - that is, when it is 
complete in the classical sense. It follows in the same way that the functor $T: \mathcal{B} \longrightarrow \mathcal{C}$ is continuous in our sense of 3.2 above exactly when it preserves all small conical limits.

For $F: \mathcal{K}^{\text {op }} \longrightarrow$ Set and $G: \mathcal{K} \longrightarrow \mathcal{B}$, the corresponding colimit-form of (3.33) is

$$
F \star G \cong \operatorname{colim}\left((\mathrm{el} F)^{\mathrm{op}} \underset{d^{\mathrm{op}}}{\longrightarrow} \mathcal{K} \underset{G}{\longrightarrow} \mathcal{B}\right)
$$

We had in (3.17), for any $\mathcal{V}$, the general expression of any $F: \mathcal{K} \longrightarrow \mathcal{V}$ as a colimit $F \star Y$ of representables; observe that when we use (3.34) to evaluate $F \star Y$ in the case $\mathcal{V}=$ Set, we re-find (3.32). Note too that, when $\mathcal{B}=$ Set, there is a second expression (3.34) for $F \star G$, since this is then isomorphic to $G \star F$ by (3.9). Finally, since the colimit of $\Delta 1: \mathcal{K} \longrightarrow$ Set is clearly the set $\pi(\mathcal{K})$ of connected components of $\mathcal{K}$, taking $G=\Delta 1$ in (3.34) and using (3.26) and (3.9) gives

$$
\operatorname{colim} F=\pi(\mathrm{el} F) \text { for } F: \mathcal{K} \longrightarrow \text { Set. }
$$

We conclude this section with some remarks about conservativeness and faithfulness for ordinary functors $T: \mathcal{A} \longrightarrow \mathcal{B}$. First, if $\mathcal{A}$ has equalizers and $T$ preserves equalizers, $T$ is faithful if it is conservative; for if $T f=T g$, the equalizer of $f$ and $g$ is sent by $T$ to an isomorphism. Next, if $\eta, \epsilon: S \dashv T: \mathcal{A} \longrightarrow \mathcal{B}$ is an adjunction, $T$ is faithful if and only if each $\epsilon_{A}: S T A \longrightarrow A$ is an epimorphism; this is immediate from (1.53), for $T_{A A^{\prime}}$ is a monomorphism exactly when each $\mathcal{A}\left(\epsilon_{A}, A^{\prime}\right)$ is. Finally, if $T$ is a right adjoint as above, and if $\mathcal{A}$ admits equalizers, then $T$ is conservative if and only if each $\epsilon_{A}$ is an extremal epimorphism (one that factorizes through no proper subobject of its codomain).

To prove this, first note that any $f: A \longrightarrow B$ in $\mathcal{A}$ which factorizes through no proper subobject of $B$ is an epimorphism, because equalizers exist. Suppose then that $T$ is conservative. If $\epsilon_{A}: S T A \longrightarrow A$ factorizes through a monomorphism $i: B \longrightarrow A$, then (by the adjunction) $1: T A \longrightarrow T A$ factorizes through the monomorphism $T i$; hence $T i$ is isomorphism, whence $i$ is. Conversely, let each $\epsilon_{A}$ be an extremal epimorphism; then, by the remark above, $T$ is faithful, so that $f: A \longrightarrow B$ is surely a monomorphism if $T f$ is an isomorphism. But in this case, by the naturality of $\epsilon$, we have $\epsilon_{B}=f . \epsilon_{A} .(S T f)^{-1}$; so that $f$ is an isomorphism.

\subsection{Full subcategories and limits; the closure of a full subcategory under a class of colimits}

Returning to the case of a general $\mathcal{V}$, we now consider some properties of (indexed) limits in relation to fully faithful functors.

A fully faithful $J: \mathcal{A} \longrightarrow \mathcal{B}$ reflects limits; in the sense that, for $F: \mathcal{K} \longrightarrow \mathcal{V}$ and $G: \mathcal{K} \longrightarrow \mathcal{A}$, a cylinder $F \longrightarrow \mathcal{A}(A, G-)$ expresses $A$ as the limit $\{F, G\}$ in $\mathcal{A}$ whenever the composite cylinder $F \longrightarrow \mathcal{A}(A, G-) \longrightarrow \mathcal{B}(J A, J G-)$ expresses $J A$ as the limit $\{F, J G\}$ in $\mathcal{B}$. This is immediate; and in the case where $\mathcal{A}$ is a full subcategory of $\mathcal{B}$, it is the assertion that $\{F, J G\}$, if it lies in $\mathcal{A}$, is also $\{F, G\}$.

If the full subcategory $\mathcal{A}$ here is reflective, and $\{F, J G\}$ exists, then the latter is isomorphic to an object of $\mathcal{A}$, which is therefore $\{F, G\}$. To see this, let the adjunction 
be $\eta, 1: S \dashv J: \mathcal{A} \longrightarrow \mathcal{B}$ and let the counit of the limit be $\mu: F \longrightarrow \mathcal{B}(\{F, J G\}, J G-)$. By (1.48) the isomorphism $\mathcal{B}(J S\{F, J G\}, J G-) \cong \mathcal{A}(S\{F, J G\}, G-) \cong \mathcal{B}(\{F, J G\}, J G-)$ is $\mathcal{B}(\eta, 1)$. By $(3.4)$, the cylinder $\mathcal{B}(\eta, 1)^{-1} \mu$ factorizes as $\mathcal{B}(f, 1) \mu$ for a unique $f: J S\{F, J G\} \longrightarrow\{F, J G\}$; and by the uniqueness part of the same result, we have $f \eta=1$. Hence $\{F, J G\}$ is a retract of the object $J S\{F, J G\}$ of $\mathcal{A}$, and so by 1.11 lies in the repletion of $\mathcal{A}$. Thus a reflective full subcategory $\mathcal{A}$ of $\mathcal{B}$ is closed under limits, and in particular is complete if $\mathcal{B}$ is so.

As for colimits in this reflective situation, if the colimit $F \star J G$ exists, $S$ preserves it by 3.2 , giving $S(F \star J G) \cong F \star S J G=F \star G$. In particular, the reflective full subcategory $\mathcal{A}$ of $\mathcal{B}$ is cocomplete if $\mathcal{B}$ is.

Consider now a family $\Phi=\left(F_{\gamma}: \mathcal{K}_{\gamma}^{\text {op }} \longrightarrow \mathcal{V}, G_{\gamma}: \mathcal{K}_{\gamma} \longrightarrow \mathcal{B}\right)_{\gamma \in \Gamma}$ of diagrams in a $\mathcal{V}$ category $\mathcal{B}$, such that each $F_{\gamma} \star G_{\gamma}$ exists. If $\mathcal{A}$ is a full subcategory of $\mathcal{B}$, consider the replete full subcategories $\mathcal{C}$ containing $\mathcal{A}$ with the property that, if any $G_{\gamma}$ takes its values in $\mathcal{C}$, then $F_{\gamma} \star G_{\gamma}$ lies in $\mathcal{C}$. The smallest such $\mathcal{C}$ is a replete full subcategory $\overline{\mathcal{A}}$ called the closure of $\mathcal{A}$ in $\mathcal{B}$ under the family $\Phi$ of colimits. For instance, we might have a set $\mathcal{F}=\left\{F: \mathcal{K}^{\mathrm{op}} \longrightarrow \mathcal{V}\right\}$ of indexing types, and take $\Phi$ to consist of all colimits that exist in $\mathcal{B}$ with indexing-type in $F$; then $\overline{\mathcal{A}}$ is the closure of $\mathcal{A}$ in $\mathcal{B}$ under colimits of type $\mathcal{F}$.

We can construct $\overline{\mathcal{A}}$ by transfinite induction: let $\mathcal{A}_{0}$ be the repletion of $\mathcal{A}$, let $\mathcal{A}_{\alpha+1}$ be $\mathcal{A}_{\alpha}$ together with all the isomorphs of those $F_{\gamma} \star G_{\gamma}$ for which $G_{\gamma}$ lands in $\mathcal{A}_{\alpha}$, and let $\mathcal{A}_{\alpha}$ be $\bigcup_{\beta<\alpha} \mathcal{A}_{\beta}$ when $\alpha$ is a limit ordinal; then the sequence is certainly stationary when the cardinal of $\alpha$ exceeds that of $\Gamma$, and gives $\overline{\mathcal{A}}$. In fact, if each ob $\mathcal{K}_{\gamma}$ has cardinal less than the regular cardinal $\rho$, we have $\overline{\mathcal{A}}=\mathcal{A}_{\rho}$, since any $\mathcal{K}_{\gamma} \longrightarrow \mathcal{A}_{\rho}$ lands in some $\mathcal{A}_{\alpha}$ for $\alpha<\rho$; in particular, if each $\mathcal{K}_{\gamma}$ is small we have $\overline{\mathcal{A}}=\mathcal{A}_{\infty}$, where $\infty$ is the cardinal of the chosen universe. It is an important observation that, when $\mathcal{A}$ is small, and each $\mathcal{K}_{\gamma}$ is small, and the number of different indexing-types $F_{\gamma}$ in $\Phi$ is small, then $\overline{\mathcal{A}}$ is small. For then the $\rho$ above is small, and the $\mathcal{A}_{\alpha}$ for $\alpha<\rho$ are small by induction: since there is but a small set of functors $\mathcal{K}_{\gamma} \longrightarrow \mathcal{A}_{\alpha}$.

If $\mathcal{B}$ itself is the $\Phi$-closure $\overline{\mathcal{A}}$ of $\mathcal{A}$, it is immediate by induction that a functor $\mathcal{A} \longrightarrow \mathcal{C}$ has, to within isomorphism, at most one extension $\mathcal{B} \longrightarrow \mathcal{C}$ that preserves the colimits in $\Phi$. However there may well be no such extension; as is clear when $\mathcal{A}$ is all of $\mathcal{B}$ and the original $\mathcal{A} \longrightarrow \mathcal{C}$ does not preserve the colimits in $\Phi$.

Proposition 3.36 Let $\mathcal{B}$ be the $\Phi$-closure of $\mathcal{A}$, and let $Q: \mathcal{B}^{\mathrm{op}} \otimes \mathcal{C} \longrightarrow \mathcal{V}$ be such that each $Q(-, C): \mathcal{B}^{\text {op }} \longrightarrow \mathcal{V}$ sends colimits belonging to $\Phi$ to limits in $\mathcal{V}$. Suppose that $\mathcal{C}$ admits all colimits indexed by the $F_{\gamma}$ occurring in $\Phi$. Then if $Q(B,-): \mathcal{C} \longrightarrow \mathcal{V}$ is representable for all $B \in \mathcal{A}$, it is representable for all $B \in \mathcal{B}$.

Proof. Let $\mathcal{D}$ be the full subcategory of all those $B$ for which a representation $Q(B, C) \cong$ $\mathcal{C}(K B, C)$ exists. Then $\mathcal{D}$ contains $\mathcal{A}$, and it remains to show that $\mathcal{D}$ contains $F_{\gamma} \star G_{\gamma}$ whenever $G_{\gamma}$ lands in $\mathcal{D}$. But then the hypothesis on $Q(-, C)$ gives $Q\left(F_{\gamma} \star G_{\gamma}, C\right) \cong$ $\left\{F_{\gamma}, Q\left(G_{\gamma}-, C\right)\right\}$, and this is $\left\{F_{\gamma}, \mathcal{C}\left(K G_{\gamma}-, C\right)\right\} \cong \mathcal{C}\left(F_{\gamma} \star K G_{\gamma}, C\right)$.

Since the hypothesis on $Q(-, C)$ in the above proposition is automatically satisfied if $Q(B, C)=\mathcal{B}(B, T C)$, we have:

Proposition 3.37 Let $T: \mathcal{C} \longrightarrow \mathcal{B}$ where $\mathcal{C}$ is cocomplete and where $\mathcal{B}$ is the closure of 
a full subcategory $\mathcal{A}$ with respect to a family of small colimits. Then $T$ has a left adjoint if $\mathcal{B}(A, T-)$ is representable for each $A \in \mathcal{A}$.

\subsection{Strongly generating functors}

In the next section we need, for ordinary categories, the notion of strong generator. Since the notion is useful for $\mathcal{V}$-categories too, we discuss it at the enriched level.

A $\mathcal{V}$-functor $T$ is said to be conservative if the underlying ordinary functor $T_{0}$ is conservative. Clearly $S T$ is conservative if $S$ and $T$ are, while if $S T$ is conservative so is $T$. Any fully-faithful $T$ is conservative, while by $(2.27)$ a functor $[M, 1]:[\mathcal{C}, \mathcal{B}] \longrightarrow[\mathcal{A}, \mathcal{B}]$ is conservative if $M: \mathcal{A} \longrightarrow \mathcal{C}$ is essentially surjective on objects. Clearly a conservative functor reflects such limits as it preserves; in the sense that if $\{F, G\}$ exists and is preserved by $T$, a cylin$\operatorname{der} F \longrightarrow \mathcal{B}(B, G-)$ is a limit-cylinder if the composite $F \longrightarrow \mathcal{B}(B, G-) \longrightarrow \mathcal{C}(T B, T G-)$ is.

If $\mathcal{A}$ is small and $Z: \mathcal{A} \longrightarrow \mathcal{B}$, we define functors

$$
\widehat{Z}: \mathcal{B}^{\text {op }} \longrightarrow[\mathcal{A}, \mathcal{V}], \quad \widetilde{Z}: \mathcal{B} \longrightarrow\left[\mathcal{A}^{\text {op }}, \mathcal{V}\right]
$$

by

$$
\widehat{Z} B=\mathcal{B}(B, Z-), \quad \widetilde{Z} B=\mathcal{B}(Z-, B) .
$$

We say that $Z$ is strongly generating if $\widetilde{Z}$ is conservative; and dually that $Z$ is strongly cogenerating if $\widehat{Z}$ is conservative. In other words, $Z$ is strongly generating if a map $f: C \longrightarrow D$ in $\mathcal{B}_{0}$ is an isomorphism whenever $\mathcal{B}(Z A, f)$ is an isomorphism in $\mathcal{V}_{0}$ for each $A \in \mathcal{A}$. This clearly depends only on the full image of $\mathcal{A}$ in $\mathcal{B}$; and is also expressed by saying that the small set $\{Z A\}_{A \in \mathcal{A}}$ of objects of $\mathcal{B}$ is a strong generator for $\mathcal{B}$. Note that the $\mathcal{V}$-functor $Z: \mathcal{A} \longrightarrow \mathcal{B}$ is certainly strongly generating when the ordinary functor $Z_{0}: \mathcal{A}_{0} \longrightarrow \mathcal{B}_{0}$ is so; for if $\mathcal{B}(Z A, f)$ is an isomorphism so is $\mathcal{B}_{0}(Z A, f)$, this being $\operatorname{VB}(Z A, f)$ by (1.33). However the converse is false; the single object $\{1\}$ is a strong generator in the Cat-category Cat but not in the Set-category Cat $_{0}$

In the case that $\mathcal{V}=$ Set we further say that $Z$ is generating, or that $\{Z A\}_{A \in \mathcal{A}}$ is a generator for $\mathcal{B}$, if $\widetilde{Z}$ is faithful; so that $f=g$ whenever $\mathcal{B}(Z A, f)=\mathcal{B}(Z A, g)$ for all $A \in \mathcal{A}$. By the remarks at the end of 3.4, a strong generator in the case $\mathcal{V}=$ Set is $a$ fortiori a generator, provided that $\mathcal{B}$ admits equalizers. In fact the above definition of a strong generator is far from ideal unless $\mathcal{B}_{0}$ admits finite limits; but we have chosen it for simplicity since this is usually the case in applications. For a general $\mathcal{V}$ it would take us into side-issues to discuss the various kinds of monomorphisms and epimorphisms in the context of $\mathcal{V}$-categories; so we refrain from defining faithfulness for a $\mathcal{V}$-functor, and hence from defining a generator for a $\mathcal{V}$-category.

We state the following proposition in the enriched case, although some remarks in 3.8 below are needed to justify its proof in that case; in the meantime we use it only for $\mathcal{V}=$ Set.

Proposition 3.40 The inclusion $Z: \mathcal{A} \longrightarrow \mathcal{B}$ of a small full subcategory is strongly generating if $\mathcal{B}$ is the closure of $\mathcal{A}$ for some set $\Phi$ of colimits. Conversely, if $Z$ is strongly 
generating, $\mathcal{B}$ is the closure of $\mathcal{A}$ for small colimits; provided that $\mathcal{B}$ is cocomplete, that $\mathcal{B}_{0}$ is finitely complete, and that each object of $\mathcal{B}_{0}$ has only a small set of extremal-epimorphic quotients.

Proof. For the direct part, let $f: C \longrightarrow D$ in $\mathcal{B}$ be such that $\mathcal{B}(A, f)$ is an isomorphism for all $A \in \mathcal{A}$. Then the full subcategory of $\mathcal{B}$ given by those $B$ for which $\mathcal{B}(B, f)$ is an isomorphism contains $\mathcal{A}$, and it clearly contains $F_{\gamma} \star G_{\gamma}$ whenever $G_{\gamma}$ lands in it; so it is all of $\mathcal{B}$, and consequently $f$ is an isomorphism.

For the converse, let $\mathcal{C}$ be the closure of $\mathcal{A}$ in $\mathcal{B}$ under small colimits. Since $\mathcal{B}$ is cocomplete, $F \star Z$ exists for all $F: \mathcal{A}^{\text {op }} \longrightarrow \mathcal{V}$; hence $\widetilde{Z}: \mathcal{B} \longrightarrow\left[\mathcal{A}^{\text {op }}, \mathcal{V}\right]$ has by $(3.5)$ the left adjoint $-\star Z:\left[\mathcal{A}^{\text {op }}, \mathcal{V}\right] \longrightarrow \mathcal{B}$. Write $R B$ for $\widetilde{Z} B \star Z$ : clearly $R B \in \mathcal{C}$. Since $(\widetilde{Z})_{0}$ is conservative, the counit $\epsilon_{B}: R B \rightarrow B$ of the adjunction is an extremal epimorphism in $\mathcal{B}_{0}$, by the remarks at the end of 3.4 .

Given any $f: C \longrightarrow D$ with $C \in \mathcal{C}$, let $u, v: E \longrightarrow C$ be its kernel-pair. By 3.8 below, $\mathcal{B}_{0}$ is cocomplete since $\mathcal{B}$ is; hence $u, v$ have a coequalizer $p_{1}: C \longrightarrow C_{1}$ in $\mathcal{B}_{0}$, and $f=f_{1} p_{1}$ for some $f_{1}: C_{1} \longrightarrow D$. Since $\epsilon_{E}$ is epimorphic, $p_{1}$ is also the coequalizer of the diagram $u \epsilon_{E}, v \epsilon_{E}: R E \longrightarrow C$ in $\mathcal{B}_{0}$; by 3.8 below again, a conical colimit in $\mathcal{B}_{0}$ is a colimit in $\mathcal{B}$ when $\mathcal{B}$ is cocomplete; hence $C_{1} \in \mathcal{C}$.

We now apply the same process to factorize $f_{1}$ as $C_{1} \longrightarrow C_{2} \longrightarrow D$ with $C_{2} \in \mathcal{C}$, so that $f$ is $C \longrightarrow C_{2} \longrightarrow D$; and continue by transfinite induction, defining $C_{\alpha}$ at a limit-ordinal $\alpha$ as the cointersection of the $C \longrightarrow C_{\beta}$ for $\beta<\alpha$. At each stage $C_{\alpha} \in \mathcal{C}$, and $f$ is $C \longrightarrow C_{\alpha} \longrightarrow D$. By the results of [41], each $C \longrightarrow C_{\alpha}$ is an extremal epimorphism in $\mathcal{B}_{0}$; so the process terminates at a small $\alpha$, since $C$ has but a small set of extremal epimorphic quotients. Yet the process can terminate only when $C_{\alpha} \longrightarrow D$ is a monomorphism; so that if $F: C \longrightarrow D$ is an extremal epimorphism, it terminates only with $C_{\alpha}=D$. We conclude that $D \in \mathcal{C}$ in this case.

Taking now for $f$ the extremal epimorphism $\epsilon_{B}: R B \longrightarrow B$ for any $B \in \mathcal{B}$, we conclude that $B \in \mathcal{C}$, as desired.

Let us now apply this, in the case of ordinary categories, to the functor $V=$ $\mathcal{V}_{0}(I,-): \mathcal{V}_{0} \longrightarrow$ Set. This functor is just the $\widetilde{Z}$ corresponding to the functor $Z: \mathbf{1} \longrightarrow \mathcal{V}_{0}$ sending the unique object of 1 to $I$; so $\{I\}$ is a generator of $\mathcal{V}_{0}$ when $V$ is faithful, and a strong generator when $V$ is conservative. Hence:

Proposition 3.41 If $\mathcal{V}_{0}$ is the closure of $\{I\}$ under small colimits, $V: \mathcal{V}_{0} \rightarrow$ Set is conservative; and the converse is true if each object of $\mathcal{V}_{0}$ has but a small set of extremal epimorphic quotients.

This last weak cowellpoweredness condition on $\mathcal{V}_{0}$ is certainly satisfied in the examples we have given with $V$ conservative, namely Set, Ab, $R$-Mod, and Ban; in fact it seems very hard to think of any complete and cocomplete locally-small category in nature that is not weakly cowellpowered, although artificial examples exist: see Example 5.3 of [36]. 


\subsection{Tensor and cotensor products}

In this section and the next three, we consider for a general $\mathcal{V}$ certain important special cases of the indexed limit $\{F, G\}$, and the extent to which the general case may be reduced to these.

The first special case is that where the domain-category $\mathcal{K}$ of $F$ and $G$ is the unit $\mathcal{V}$-category $\mathcal{I}$, so that $F: \mathcal{I} \longrightarrow \mathcal{V}$ is in effect an object $X$ of $\mathcal{V}$ and $G: \mathcal{I} \longrightarrow \mathcal{B}$ is an object $C$ of $\mathcal{B}$. Then the object $\{F, G\}$ of $\mathcal{B}$ is written $X \pitchfork C$, or sometimes $[X, C]$, and is called the cotensor product in $\mathcal{B}$ of $X$ and $C$. By (3.1), its defining equation is a $\mathcal{V}$-natural isomorphism

$$
\mathcal{B}(B, X \pitchfork C) \cong[X, \mathcal{B}(B, C)]
$$

with counit

$$
X \rightarrow \mathcal{B}(X \pitchfork C, C) .
$$

If $X \pitchfork C$ exists for all $X \in \mathcal{V}$ and all $C \in \mathcal{B}$, the $\mathcal{V}$-category $\mathcal{B}$ is said to admit cotensor products, or to be cotensored. Because $\mathcal{I}$ is small, any complete $\mathcal{B}$ is cotensored. Since by (1.27) we have the $\mathcal{V}$-natural isomorphism $[Y,[X, Z]] \cong[X,[Y, Z]]$ in $\mathcal{V}$, we conclude that $\mathcal{V}$ is cotensored, the cotensor product $X \pitchfork Z$ being just the internal-hom $[X, Z]$. When $\mathcal{V}=$ Set, the cotensor product $X \pitchfork C$ in $\mathcal{B}$ is clearly just the power $C^{X}$ - the product of $X$ copies of the object $C$ of $\mathcal{B}$.

The dual notion is the tensor product $X \otimes C \in \mathcal{B}$ of $X \in \mathcal{V}$ and $C \in \mathcal{B}$, defined by

$$
\mathcal{B}(X \otimes C, B) \cong[X, \mathcal{B}(C, B)] .
$$

Using (1.27) again, we have that $\mathcal{V}$ is tensored, $X \otimes Y$ being the ordinary tensor product in $\mathcal{V}$. When $\mathcal{V}=$ Set, the tensor product $X \otimes C$ is just the copower $X \cdot C$ - the coproduct in $\mathcal{B}$ of $X$ copies of the object $C$.

The natural bijection

$$
\mathcal{B}_{0}(B, X \pitchfork C) \cong \mathcal{V}_{0}(X, \mathcal{B}(B, C))
$$

obtained by applying $V$ to (3.42) corresponds to (3.4), and is in general insufficient to ensure that $X \pitchfork C$ is the cotensor product of $X$ and $C$, unless this latter is known to exist. For instance, take $\mathcal{V}=$ Cat and $X=\mathbf{2}$, and let $\mathcal{B}$ be the 2-category generated by the 2-graph

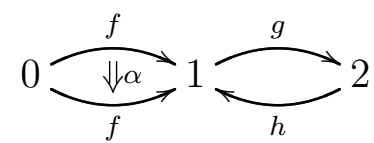

with the relations $g \alpha=1$ and $g h=1$. If we were to define $\mathbf{2} \pitchfork 2$ to be 1 , with as counit (3.43) the functor from 2 corresponding to the map $1_{g}: g \rightarrow g$, we should satisfy (3.45) but not (3.42).

When $V$ is conservative, however, a map (3.43) that induces a bijection (3.45) also induces an isomorphism (3.42), as we saw in 1.11. In fact we have:

Proposition 3.46 Let $V$ be conservative, and let each object of $\mathcal{V}_{0}$ have but a small set of extremal-epimorphic quotients. Then a $\mathcal{V}$-category $\mathcal{B}$ is cotensored if the ordinary category $\mathcal{B}_{0}$ is complete. 
Proof. For a fixed $C \in \mathcal{B}$ we apply Proposition 3.37 to the ordinary functor $\mathcal{B}(-, C)_{0}: \mathcal{B}_{0}^{\text {op }} \longrightarrow \mathcal{V}_{0}$; observing that $\mathcal{V}_{0}$ is the closure of $\{I\}$ under small colimits by Proposition 3.41, while $\mathcal{V}_{0}(I, \mathcal{B}(-, C))$ is representable as $\mathcal{B}_{0}(-, C)$. This gives a natural bijection (3.45), the counit (3.43) of which then induces as above a natural isomorphism (3.42) because $V$ is conservative.

When $\mathcal{B}$ is cotensored, a third term can be added to the equation (3.22) expressing the continuity of a limit in its index; using a proof like that of (3.18), we easily get

$$
\{F \star G, T\} \cong\{(G-) ?, F-\pitchfork T ?\} \cong\{F,\{G-, T\}\}
$$

where $F-\pitchfork T$ ? is the functor sending $(K, A)$ to $F K \pitchfork T A$; here the first isomorphism in (3.47) holds under the hypothesis that $F \star G$ exists, and the second under the hypothesis that each $\{G K, T\}$ exists. The corresponding extension of (3.23), now supposing $\mathcal{B}$ to be tensored, is

$$
(F \star G) \star T \cong(G-) ? \star(F \otimes T) \cong F \star(G-\star T) .
$$

\subsection{Conical limits in a $\mathcal{V}$-category}

For the next special case of a limit $\{F, G\}$, we begin with a $\mathcal{V}$-category $\mathcal{B}$, a locally-small ordinary category $\mathcal{L}$, and a functor $T: \mathcal{L} \longrightarrow \mathcal{B}_{0}$. We take for $\mathcal{K}$ the free $\mathcal{V}$-category $\mathcal{L}_{\mathcal{V}}$ on $\mathcal{L}$ as in 2.5, for $G: \mathcal{K} \longrightarrow \mathcal{B}$ the $\mathcal{V}$-functor corresponding in 2.5 to $T: \mathcal{L} \longrightarrow \mathcal{B}_{0}$, and for $F: \mathcal{K} \longrightarrow \mathcal{V}$ the $\mathcal{V}$-functor corresponding to the ordinary functor $\Delta I: \mathcal{L} \longrightarrow \mathcal{V}_{0}$ constant at $I \in \mathcal{V}_{0}$. Observe that, since the adjunction of 2.5 is of course 2 -natural, $\mathcal{B}(B, G-): \mathcal{K} \longrightarrow \mathcal{V}$ is the $\mathcal{V}$-functor corresponding to the ordinary functor

$$
\mathcal{L} \underset{T}{\longrightarrow} \mathcal{B}_{0} \underset{\mathcal{B}(B,-)_{0}}{\longrightarrow} \mathcal{V}_{0}
$$

which we may loosely call $\mathcal{B}(B, T-)$.

A family $\alpha_{K}: X \longrightarrow[F K, \mathcal{B}(B, G K)]$ is $\mathcal{V}$-natural by $1.8(\mathrm{~m})$ if and only if the corresponding $\bar{\alpha}_{K}: F K \longrightarrow[X, \mathcal{B}(B, G K)]$ is so; by 2.5 this is equivalent to the mere naturality of $\bar{\alpha}_{K}:(\Delta I) K \longrightarrow[X, \mathcal{B}(B, T K)]$, and hence to the requirement that $\alpha_{K}: X \longrightarrow[I, \mathcal{B}(B, T K)] \cong \mathcal{B}(B, T K)$ be a cone over the ordinary functor $\mathcal{B}(B, T-)$ of (3.49). It follows from (2.10) that

$$
[\mathcal{K}, \mathcal{V}](F, \mathcal{B}(B, G-)) \cong \lim \mathcal{B}(B, T-),
$$

this being the ordinary conical limit in $\mathcal{V}_{0}$ of the ordinary functor $(3.49)$ - the identification of which with the left side of (3.50) exhibits it as being $\mathcal{V}$-functorial in $B$.

In this situation we call $\{F, G\}$, if it exists, the (conical) limit in $\mathcal{B}$ of $T: \mathcal{L} \longrightarrow \mathcal{B}_{0}$, denoting it formally by $\lim _{\mathcal{B}} T$, but usually in practice simply by $\lim T$. By (3.1) and (3.50), its defining equation is the $\mathcal{V}$-natural isomorphism

$$
\mathcal{B}(B, \lim T) \cong \lim \mathcal{B}(B, T-)
$$

in $\mathcal{V}_{0}$, the counit $\mu$ of (3.2) here reducing to the limit cone

$$
\mu_{K}: \lim T \longrightarrow T K .
$$


Since the representable $V=\mathcal{V}_{0}(I,-): \mathcal{V}_{0} \longrightarrow$ Set preserves limits, applying $V$ to $(3.51)$ gives the natural bijection

$$
\mathcal{B}_{0}(B, \lim T) \cong \lim \mathcal{B}_{0}(B, T-),
$$

showing by (3.29) that $\lim _{\mathcal{B}} T$, if it exists, with the cone (3.52), is also the ordinary conical limit $\lim _{\mathcal{B}_{0}} T$ of the ordinary functor $T: \mathcal{L} \longrightarrow \mathcal{B}_{0}$. It is for this reason that we write simply $\lim T$; but we distinguish verbally the existence (3.51) of $\lim T$ in $\mathcal{B}$ from the (mere) existence (3.53) of $\lim T$ in $\mathcal{B}_{0}$. The latter is in general strictly weaker, as we remarked in (3.4); for instance, if $\mathcal{V}=$ Cat and $\mathcal{B}$ is the 2-category freely generated by the 2-graph

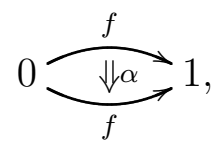

then the cone $1 \prec 1 \longrightarrow 1$ exhibits 1 as the product $1 \times 1$ in $\mathcal{B}_{0}$, but not in $\mathcal{B}$. Note that an equivalent way of expressing the existence in $\mathcal{B}$ of $\lim T$ is to say that $\lim T$ exists in $\mathcal{B}_{0}$ and is preserved by all the functors $\mathcal{B}(B,-)_{0}: \mathcal{B}_{0} \longrightarrow \mathcal{V}_{0}$.

The difference between the existence in $\mathcal{B}$ and that in $\mathcal{B}_{0}$ of such conical limits vanishes, of course, if $V$ is conservative. It also vanishes for any $\mathcal{V}$ if $\mathcal{B}$ is tensored. To see this we have only to replace $B$ in (3.53) by $X \otimes B$ for any $X \in \mathcal{V}$, to apply the dual of (3.45), and to recall that $\mathcal{V}_{0}(X,-)$ preserves limits; we conclude that each $\mathcal{V}_{0}(X,-)$ carries the map $\mathcal{B}(B, \lim T) \longrightarrow \lim \mathcal{B}(B, T-)$ induced by $\mu$ into an isomorphism; whence this map is itself an isomorphism.

In particular the difference vanishes if $\mathcal{B}=\mathcal{V}$, so that $\mathcal{V}$ admits all small conical limits. Note that the right side of (3.51), which until now was the limit in $\mathcal{V}_{0}$, is in fact also the limit in $\mathcal{V}$. The difference also vanishes if $\mathcal{B}=\mathcal{V}^{\mathrm{op}}$, since $\mathcal{V}^{\mathrm{op}}$ is tensored because $\mathcal{V}$ is cotensored; hence $\mathcal{V}$ admits all small conical colimits. Here the notion of conical colimit is the evident dual to that of conical limit, defined for $T: \mathcal{L} \longrightarrow \mathcal{B}_{0}$ by

$$
\mathcal{B}(\operatorname{colim} T, B) \cong \lim \mathcal{B}(T-, B) .
$$

Since $\mathcal{L}_{\mathcal{V}}$ is small when $\mathcal{L}$ is, any complete $\mathcal{B}$ admits all small conical limits. In consequence, by $3.4, \mathcal{B}_{0}$ is complete whenever $\mathcal{B}$ is. However the existence in $\mathcal{B}$ of all small conical limits does not suffice, in general, for completeness of $\mathcal{B}$. Take $\mathcal{V}=\mathbf{C a t}$, and let $\mathcal{B}$ be the full sub-2-category of Cat determined by all categories with at most one object. Since $\mathcal{V}$ admits all small conical limits so does $\mathcal{B}$, for the conical limit in $\mathcal{V}$ of a diagram in $\mathcal{B}$ clearly lies in $\mathcal{B}$. Yet $\mathcal{B}$ is not complete, for $\mathbf{2} \pitchfork B$ does not exist in $\mathcal{B}$ if $B$ has more than one map.

\subsection{The inadequacy of conical limits}

This inadequacy of conical limits deserves some further comment. It shows that, for a general $\mathcal{V}$, there can be no analogue of (3.32) allowing us to express, as in (3.33), a general limit as a conical one. In fact, as in 3.4, any $F: \mathcal{K} \longrightarrow \mathcal{V}$ is the vertex of a canonical inductive cone in $[\mathcal{K}, \mathcal{V}]_{0}$, whose generators are all the maps $\mathcal{K}(K,-) \rightarrow F$ from representables 
into $F$. Since such maps correspond by Yoneda to elements $x \in \mathcal{V}_{0}(I, F K)=V F K$, the base of this cone is a functor with domain el $\left(V F_{0}\right)^{\text {op }}$, the dual of the category of elements of the ordinary functor

$$
\mathcal{K}_{0} \underset{F_{0}}{\longrightarrow} \mathcal{V}_{0} \underset{V}{\longrightarrow} \text { Set. }
$$

When $\mathcal{K}$ is small so is $\operatorname{el}\left(V F_{0}\right)$, so that this functor el $\left(V F_{0}\right)^{\text {op }} \longrightarrow[\mathcal{K}, \mathcal{V}]_{0}$ has a conical colimit in $[\mathcal{K}, \mathcal{V}]$; for $[\mathcal{K}, \mathcal{V}]$ admits small conical colimits pointwise since $\mathcal{V}$ admits them. We therefore have a comparison map

$$
\left\{\operatorname{colim}\left(\mathrm{el}\left(V F_{0}\right)^{\mathrm{op}} \underset{d^{\mathrm{op}}}{\longrightarrow} \mathcal{K}_{0}^{\mathrm{op}} \underset{Y_{0}}{\longrightarrow}[\mathcal{K}, \mathcal{V}]_{0}\right)\right\} \longrightarrow F
$$

in $[\mathcal{K}, \mathcal{V}]_{0}$. However, in contrast to the case $\mathcal{V}=$ Set, the map (3.56) is not in general an isomorphism - even when $V$ is conservative.

To see this it suffices to take for $\mathcal{K}$ the unit $\mathcal{V}$-category $\mathcal{I}$, so that $F$ is just an object $X$ of $\mathcal{V}$. The canonical cone is now the cone in $\mathcal{V}_{0}$ given by all the maps $I \longrightarrow X$. To ask that this be a colimit-cone for each $X$ is to ask that $\{I\}$ be dense in $\mathcal{V}_{0}$, in the sense of 5.1 below; this is stronger than asking $V$ to be conservative, which by 3.6 is to ask that $\{I\}$ be a strong generator for $\mathcal{V}_{0}$; it is false even for $\mathcal{V}=\mathbf{A b}$. It is of course true for $\mathcal{V}=$ Set, and for certain other $\mathcal{V}$ : for instance, sets on which a commutative monoid acts, with a tensor product analogous to that for modules over a commutative ring.

What is true when $V$ is conservative, by Proposition 3.41, is that any $X \in \mathcal{V}_{0}$, although not the canonical colimit of the maps from $I$, is in all reasonable cases in the closure of $\{I\}$ under small colimits; it is, so to speak, an iterated colimit of $I$. In this case an arbitrary $F: \mathcal{K} \longrightarrow \mathcal{V}$ is an iterated colimit of representables; and so the existence of all small conical limits in $\mathcal{B}$ does imply the existence of all indexed limits $\{F, G\}$, as we shall show formally in 3.10 below - even though $\{F, G\}$ cannot be expressed as a single conical limit.

In the study of enriched universal algebra, it is an important observation that, for many $\mathcal{V}$ including Cat and $\mathbf{G p d},(3.56)$ is an isomorphism whenever $F$ is left exact; see $[45]$.

Before ending this section, we look at the possible existence in certain cases of more general conical limits. The notion of a cone involves the notion of a constant functor; yet in general there is no such thing as "the $\mathcal{V}$-functor $\mathcal{K} \longrightarrow \mathcal{B}$ constant at $B \in \mathcal{B}$ ". The object $B \in \mathcal{B}$ may be seen as a $\mathcal{V}$-functor $\mathcal{I} \longrightarrow \mathcal{B}$, but there is no canonical $\mathcal{V}$-functor $\mathcal{K} \longrightarrow \mathcal{I}$; in fact there may well be none at all. There is certainly a unique $V$-functor $\mathcal{K} \longrightarrow 1$, where 1 is the terminal $\mathcal{V}$-category with one object 0 and with, for hom-object $1(0,0)$, the terminal object 1 of $\mathcal{V}_{0}$; but there is no $\mathcal{V}$-functor $1 \rightarrow \mathcal{I}$ unless $I$ is a retract of 1 in $\mathcal{V}_{0}$ - which is false for $\mathcal{V}=R$-Mod, DG- $R$-Mod, CGTop , $_{*}$, and so on. This is why we have defined conical limits as above, taking for $\mathcal{K}$ a free $\mathcal{V}$-category $\mathcal{L}_{\mathcal{V}}$; for there are always constant ordinary functors $\mathcal{L} \longrightarrow \mathcal{B}_{0}$.

Yet in the special case of cartesian closed $\mathcal{V}$ (and in some other cases as well), it happens that $I=1$ in $\mathcal{V}_{0}$, so that the $\mathcal{V}$-categories $\mathcal{I}$ and 1 coincide; then there is for any $B \in \mathcal{B}$ a "constant $\mathcal{V}$-functor" $\Delta B: \mathcal{K} \longrightarrow 1 \longrightarrow \mathcal{B}$ with this value. In particular there is a constant $\mathcal{V}$-functor $\Delta 1: \mathcal{K} \longrightarrow \mathcal{V}$, and we can consider for any $G: \mathcal{K} \longrightarrow \mathcal{B}$ the indexed 
limit $\{\Delta 1, G\}$. If this exists it represents $[\mathcal{K}, \mathcal{V}](\Delta 1, \mathcal{B}(B, G-))$; which is easily seen to be $[\mathcal{K}, \mathcal{B}](\Delta B, G)$, the isomorphism $\left[(\Delta 1) K, \mathcal{B}\left(B, G K^{\prime}\right)\right] \cong \mathcal{B}\left((\Delta B) K, G K^{\prime}\right)$ being clearly $\mathcal{V}$-natural in all three variables. Thus we are led, when $\mathcal{V}$ is cartesian closed, to define the conical limit $\lim G$ by

$$
\lim G=\{\Delta 1, G\}
$$

for any $\mathcal{V}$-functor $G: \mathcal{K} \longrightarrow \mathcal{B}$; so that $\lim G$ is the representing object in

$$
\mathcal{B}(B, \lim G) \cong[\mathcal{K}, \mathcal{B}](\Delta B, G) .
$$

This reduces to $\lim T$ when $G: \mathcal{L}_{\mathcal{V}} \longrightarrow \mathcal{B}$ is the $\mathcal{V}$-functor corresponding to the ordinary functor $T: \mathcal{L} \longrightarrow \mathcal{B}_{0}$; and thus it extends the notion of conical limit from functors to $\mathcal{V}$-functors, for cartesian closed $\mathcal{V}$.

By $(3.57), \lim G$ certainly exists for every $\mathcal{V}$-functor $G: \mathcal{K} \longrightarrow \mathcal{B}$ with $\mathcal{K}$ small, when $\mathcal{V}$ is cartesian closed, if the $\mathcal{V}$-category $\mathcal{B}$ is complete. Even the existence of these more general conical limits, however, does not conversely imply completeness of $\mathcal{B}$. The example in 3.8 above of a non-complete $\mathcal{B}$ when $\mathcal{V}=\mathbf{C a t}$, given by the full subcategory of Cat determined by the categories with at most one object, clearly admits all such conical limits indexed by a 2-category.

\subsection{Ends and coends in a general $\mathcal{V}$-category; completeness}

For the final special case of a limit $\{F, G\}$ we take the indexing-type $F: \mathcal{K} \longrightarrow \mathcal{V}$ to be $\operatorname{Hom}_{\mathcal{A}}: \mathcal{A}^{\mathrm{op}} \otimes \mathcal{A} \longrightarrow \mathcal{V}$ for some $\mathcal{V}$-category $\mathcal{A}$. Then a diagram $G$ in $\mathcal{B}$ of this type is just a $\mathcal{V}$-functor $G: \mathcal{A}^{\text {op }} \otimes \mathcal{A} \longrightarrow \mathcal{B}$. If $\left\{\operatorname{Hom}_{\mathcal{A}}, G\right\}$ exists we denote it by

$$
\int_{A \in \mathcal{A}} G(A, A)=\left\{\operatorname{Hom}_{\mathcal{A}}, G\right\}
$$

and call it the end of $G$. By the extra-variable Yoneda isomorphism (2.38), the right side of (3.1) may here be written as $\int_{A} \mathcal{B}(B, G(A, A))$, this being the end of a bifunctor $\mathcal{A}^{\text {op }} \otimes \mathcal{A} \longrightarrow \mathcal{V}$ as defined in 2.1. Hence the defining equation of the end $\int_{A} G(A, A)$ in $\mathcal{B}$ is

$$
\mathcal{B}\left(B, \int_{A} G(A, A)\right) \cong \int_{A} \mathcal{B}(B, G(A, A))
$$

while the counit $\mu$ of $(3.2)$ corresponds to a $\mathcal{V}$-natural family

$$
\lambda_{A}: \int_{A} G(A, A) \longrightarrow G(A, A) .
$$

It is immediate from (2.3) that, when $\mathcal{B}=\mathcal{V}$, the end $\int_{A} G(A, A)$ in the sense of 2.1 is also the end in the present sense, so that there is no ambiguity; indeed (3.60) is just one more illustration of the fact that limits in $\mathcal{B}$ are defined representably in terms of limits in $\mathcal{V}$.

Applying $V=\mathcal{V}_{0}(I,-)$ to (3.60), and observing that $\mathcal{V}_{0}\left(I, \int_{A} \mathcal{B}(B, G(A, A))\right)$ is by 2.1 the set of $\mathcal{V}$-natural families $I \longrightarrow \mathcal{B}(B, G(A, A))$, which is in effect the set $\mathcal{V}$-nat $(B, G(A, A))$ of $\mathcal{V}$-natural families $B \longrightarrow G(A, A)$, we have a bijection of sets

$$
\mathcal{B}_{0}\left(B, \int_{A} G(A, A)\right) \cong \mathcal{V}-\operatorname{nat}(B, G(A, A))
$$


showing that the counit $\lambda$ of (3.61) is the universal $\mathcal{V}$-natural family with codomain $G(A, A)$. When $\mathcal{B}=\mathcal{V}$, this universal property sufficed in 2.1 to define $\int_{A} G(A, A)$; but in general it does not. For instance, if $\mathcal{V}=$ Cat and $\mathcal{A}$ is the discrete 2-category 2, then $\int_{A} G(A, A)$ is just the product $G(0,0) \times G(1,1)$, which for suitable $G$ can be any product in $\mathcal{B}$; so that (3.54) again provides a counter-example. Of course this universal property does suffice to detect $\int_{A} G(A, A)$ if this is known to exist; and it does suffice to define it if $V$ is conservative, or if $\mathcal{B}$ is tensored - the argument for this last being like the argument for the analogous fact in 3.8 .

The general Fubini and interchange-of-limits theorems of 3.3 have as particular cases, under the hypothesis that the inner limits exist, such results as

$$
\begin{aligned}
\int_{A} \int_{B} G(A, A, B, B) & \cong \int_{(A, B)} G(A, A, B, B) \cong \int_{B} \int_{A} G(A, A, B, B), \\
\int_{A} \lim _{\alpha} G_{\alpha}(A, A) & \cong \lim _{\alpha} \int_{A} G_{\alpha}(A, A), \\
\int_{A} X \pitchfork G(A, A) & \cong X \pitchfork \int_{A} G(A, A) ;
\end{aligned}
$$

there is no point in specifically remembering any but the general results of 3.3.

The dual notion is that of the coend $\int^{A} G(A, A)$ of $G: \mathcal{A}^{\mathrm{op}} \otimes \mathcal{A} \longrightarrow \mathcal{B}$, defined by

$$
\int{ }^{A} G(A, A)=\operatorname{Hom}_{\mathcal{A}} \star G,
$$

and hence determined by

$$
\mathcal{B}\left(\int^{A} G(A, A), B\right) \cong \int_{A} \mathcal{B}(G(A, A), B) .
$$

We now observe that any $\mathcal{B}$ which admits cotensor products and small conical limits admits every end $\int_{A \in \mathcal{A}} G(A, A)$ with small $\mathcal{A}$. For let us temporarily define an object $\int_{A} G(A, A)$ as the equalizer of the evident pair $\rho, \sigma$ of maps (cf. (2.2)) in

$$
\int_{A \in \mathcal{A}} G(A, A) \underset{\lambda}{\longrightarrow} \prod_{A \in \mathcal{A}} G(A, A) \underset{\sigma}{\stackrel{\rho}{\longrightarrow}} \prod_{A, C \in \mathcal{A}} \mathcal{A}(A, C) \pitchfork G(A, C) .
$$

Applying the limit-preserving $\mathcal{B}(B,-)$ to (3.68), using (3.42), and comparing the result with (2.2), we get precisely the desired (3.60).

We next observe that, for any $F: \mathcal{K} \longrightarrow \mathcal{V}$ and $G: \mathcal{K} \longrightarrow \mathcal{B}$, we have

$$
\{F, G\} \cong \int_{K} F K \pitchfork G K \quad \text { if } \mathcal{B} \text { is cotensored; }
$$

or even if only these particular cotensor products $F K \pitchfork G K$ exist: either side of (3.69) existing if the other does. For the right side of (3.1) is $\int_{K}[F K, \mathcal{B}(B, G K)]$ by (2.10), which here is $\int_{K} \mathcal{B}(B, F K \pitchfork G K)$ by (3.42). The dual of this is

$$
F \star G \cong \int^{K} F K \otimes G K \quad \text { if } \mathcal{B} \text { is tensored } .
$$

Note that the Yoneda isomorphisms (3.10) for cotensored [resp. tensored] $\mathcal{B}$ take the forms

$$
\int_{L} \mathcal{K}(K, L) \pitchfork G L \cong G K, \quad \int^{L} \mathcal{K}(L, K) \otimes G L \cong G K
$$


while the expression $(3.17)$ of an arbitrary $F: \mathcal{K} \longrightarrow \mathcal{V}$ as a colimit of representables takes the form

$$
\int^{L} \mathcal{K}(L,-) \otimes F L \cong F
$$

The two observations above give at once:

Theorem 3.73 The $\mathcal{V}$-category $\mathcal{B}$ is complete if and only if it admits cotensor products and all small conical limits. When this is so, the $\mathcal{V}$-functor $T: \mathcal{B} \longrightarrow \mathcal{C}$ is continuous if and only if it preserves cotensor products and small conical limits.

We have already seen in 3.2 that $\mathcal{V}$ is complete. Since $\mathcal{V}$ admits tensor products by 3.7 and small conical colimits by 3.8 , we conclude that

$$
\mathcal{V} \text { is cocomplete. }
$$

By 3.3 and 3.5, we have:

Proposition 3.75 If $\mathcal{A}$ is small, every reflective full subcategory of $[\mathcal{A}, \mathcal{V}]$ is complete and cocomplete.

It is a matter of common experience that, in the case $\mathcal{V}=\mathbf{A b}$ of additive categories, it has never proved necessary to distinguish the completeness of $\mathcal{B}$ from that of $\mathcal{B}_{0}$. In fact Proposition 3.46, along with the observation in 3.8 that conical limits in $\mathcal{B}_{0}$ are also limits in $\mathcal{B}$ when $V$ is conservative, gives:

Proposition 3.76 Let $V$ be conservative, and let each object of $\mathcal{V}_{0}$ have but a small set of extremal-epimorphic quotients. Then a $\mathcal{V}$-category $\mathcal{B}$ is complete whenever the ordinary category $\mathcal{B}_{0}$ is so.

We finally observe that (3.68) and (3.69) do not "reduce all indexed limits to conical limits and cotensor products"; the $\{F, G\}$ in (3.69) may well exist although the $F K \pitchfork G K$ do not, an example being provided by the Yoneda isomorphisms (3.10) for an arbitrary $\mathcal{B}$. It would be equally false for ordinary categories to say that "all conical limits reduce to products and equalizers"; there all conical limits exist if all products and equalizers do, but a particular pullback may well exist even though the product of which it would be a subobject does not. The moral is that it is proper to recognize the most general (indexed) limit concept, while seeing the results above as simple tests for completeness.

\subsection{The existence of a limit-preserving universe-enlargement $\mathcal{V} \subset \mathcal{V}^{\prime}$}

We now justify our assertion of 2.6, that every $\mathcal{V}$ has a well-behaved extension to a higher-universe version $\mathcal{V}^{\prime}$. The analysis below is a very special case of that in Day's consideration [12] of monoidal biclosed structures on enriched functor categories.

Given a set $\{\mathcal{A}\}$ of $\mathcal{V}$-categories $\mathcal{A}$ that we should like to be small when seen as $\mathcal{V}^{\prime}$-categories, let Set $^{\prime}$ be the category of sets in some universe containing as elements $\operatorname{ob}($ Set $)$, ob $\mathcal{V}$, and ob $\mathcal{A}$ for each $\mathcal{A} \in\{\mathcal{A}\}$.

Take $\mathcal{V}_{0}^{\prime}=\left[\mathcal{V}_{0}^{\text {op }}\right.$, Set $\left.^{\prime}\right]$, so that $\mathcal{V}_{0}^{\prime}$ is a Set $^{\prime}$-category, complete and cocomplete in

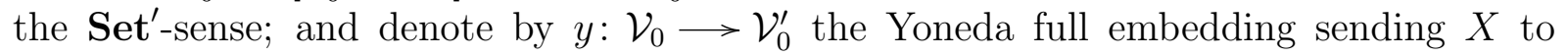


$\mathcal{V}_{0}(-, X)$. Then there is an essentially unique symmetric monoidal closed structure $\mathcal{V}^{\prime}=$ $\left(\mathcal{V}_{0}^{\prime}, \otimes^{\prime}, I^{\prime},[,]^{\prime}\right)$ on $\mathcal{V}_{0}^{\prime}$ extending that on $\mathcal{V}_{0}$

First observe that, since the left adjoint functors $F \otimes^{\prime}-$ and $-\otimes^{\prime} G$ must preserve all colimits, and since $F$ is by (3.17) the colimit $F \star y$ of representables, and similarly for $G$, we must have $F \otimes^{\prime} G \cong(F \star y) \otimes^{\prime} G \cong F \star\left(y \otimes^{\prime} G\right) \cong F \star\left(G \star\left(y \otimes^{\prime} y\right)\right)$, which by (3.21) is $(F \times G) \star\left(y \otimes^{\prime} y\right)$. Since $\otimes^{\prime}$ is to extend $\otimes$, we must have

$$
y X \otimes^{\prime} y Y=\mathcal{V}_{0}(-, X) \otimes^{\prime} \mathcal{V}_{0}(-, Y) \cong \mathcal{V}_{0}(-, X \otimes Y)=y(X \otimes Y)
$$

Thus we are forced to define $F \otimes^{\prime} G$ as $(F-\times G ?) \star y(-\otimes ?)$, which written in the more explicit coend form (3.70) is

$$
F \otimes^{\prime} G=\int^{X, Y} F X \times G Y \times \mathcal{V}_{0}(-, X \otimes Y)
$$

With this definition, moreover, (3.77) is clearly satisfied, by Yoneda in the form (3.71).

The definition (3.5) of $\star$ applied to $F \otimes^{\prime} G=(F-\times G$ ? $) \star y\left(-\otimes\right.$ ?) gives $\mathcal{V}_{0}^{\prime}\left(F \otimes^{\prime} G, H\right) \cong$ $\left[\mathcal{V}_{0}^{\text {op }} \times \mathcal{V}_{0}^{\text {op }}, \operatorname{Set}^{\prime}\right]\left(F-\times G ?, \mathcal{V}_{0}^{\prime}(y(-\otimes ?), H)\right)$. But $\mathcal{V}_{0}^{\prime}(y(-\otimes ?), H) \cong H(-\otimes ?)$ by Yoneda, so that $\mathcal{V}_{0}^{\prime}\left(F \otimes^{\prime} G, H\right) \cong\left[\mathcal{V}_{0}^{\text {op }} \times \mathcal{V}_{0}^{\text {op }}\right.$, $\left.\operatorname{Set}^{\prime}\right](F-\times G$ ?, $H(-\otimes$ ? $))$, which by $(2.29)$ is also $\mathcal{V}_{0}^{\prime}\left(F, \mathcal{V}_{0}^{\prime}(G\right.$ ? $\left.H(-\otimes ?))\right)$. In other words $-\otimes^{\prime} G$ has as desired a right adjoint $[G,]^{\prime}$ :

$$
\mathcal{V}_{0}^{\prime}\left(F \otimes^{\prime} G, H\right) \cong \mathcal{V}_{0}^{\prime}\left(F,[G, H]^{\prime}\right)
$$

where

$$
[G, H]^{\prime}=\mathcal{V}_{0}^{\prime}(G ?, H(-\otimes ?))=\int_{Y}[G Y, H(-\otimes Y)],
$$

[ , ] here denoting the internal-hom in $\mathbf{S e t}^{\prime}$. Note that $\left[\mathcal{V}_{0}(-, X), \mathcal{V}_{0}(-, Z)\right]^{\prime}=$ $\int_{Y}\left[\mathcal{V}_{0}(Y, X), \mathcal{V}_{0}(-\otimes Y, Z)\right]$ is, by the form $(3.71)$ of Yoneda, $\mathcal{V}_{0}(-\otimes X, Z) \cong \mathcal{V}_{0}(-,[X, Z])$ Thus

$$
[y X, y Z]^{\prime} \cong y[X, Z]
$$

so that $[,]^{\prime}$ is an extension of the $[$,$] of \mathcal{V}$.

From the existence of this right adjoint and the evident symmetry of $\otimes^{\prime}$, it follows that $\left(F \otimes^{\prime} G\right) \otimes^{\prime} H$ preserves colimits in each variable separately; so that from the colimitexpressions of the form $F \cong F \star y$ and from (3.77), we get

$$
\left(F \otimes^{\prime} G\right) \otimes^{\prime} H \cong \int^{X, Y, Z} F X \times G Y \times H Z \times \mathcal{V}_{0}(-,(X \otimes Y) \otimes Z),
$$

with a similar expression for $F \otimes^{\prime}\left(G \otimes^{\prime} H\right)$. Hence the associativity isomorphism for $\otimes$ induces one for $\otimes^{\prime}$ as in (2.4); and similarly of course for the commutativity. Again, if we set

$$
I^{\prime}=y I=\mathcal{V}_{0}(-, I),
$$

the cocontinuity in $F$ of $F \otimes^{\prime} I^{\prime}$, along with (3.77), gives an evident isomorphism $F \otimes^{\prime} I^{\prime} \cong$ $F$.

Verification of the coherence conditions in $\mathcal{V}^{\prime}$ from those in $\mathcal{V}$ is straightforward: the essential logical point is the uniqueness of the induced map $\int_{A} \alpha_{A A}$ in (2.4). A careful discussion is given by Day in [12], and a more streamlined one in his thesis [13]. 
Note that the derivation of (3.79) made no use of the closed structure of the symmetric monoidal $\mathcal{V}$. This proves our assertion at the end of 1.4 above, that any symmetric monoidal category can be embedded in a closed one.

An essential property of the embedding $y: \mathcal{V}_{0} \longrightarrow \mathcal{V}_{0}^{\prime}$ is that it preserves all limits that exist in $\mathcal{V}_{0}$; in fact these become pointwise limits in $\mathcal{V}_{0}^{\prime}$, for the composite with $y$ of the evaluation $E_{X}: \mathcal{V}_{0}^{\prime} \longrightarrow$ Set $^{\prime}$ is $\mathcal{V}_{0}(X,-): \mathcal{V}_{0} \rightarrow$ Set $\rightarrow$ Set $^{\prime}$, which preserves all limits. It then follows from (2.1) that the end in $\mathcal{V}$ of $T: \mathcal{A}^{\text {op }} \otimes \mathcal{A} \longrightarrow \mathcal{V}$, if it exists, coincides with the end in $\mathcal{V}^{\prime}$ of $\mathcal{A}^{\text {op }} \otimes \mathcal{A} \longrightarrow \mathcal{V} \longrightarrow \mathcal{V}^{\prime}$; so that $\int_{A} T(A, A)$ is unambiguous for such a functor. In consequence, the definition $(2.10)$ of $[\mathcal{A}, \mathcal{B}](T, S)$ is also unambiguous: if it exists as an object of $\mathcal{V}$, it has this same value when $\mathcal{A}$ and $\mathcal{B}$ are thought of as $\mathcal{V}^{\prime}$-categories.

This justifies the alternative view mentioned in 3.1 of the definition (3.1) of $\{F, G\}$ for $F: \mathcal{K} \longrightarrow \mathcal{V}$ and $G: \mathcal{K} \longrightarrow \mathcal{B}$ : namely that $[\mathcal{K}, \mathcal{V}]$ always exists, even if $\mathcal{K}$ is large, as a $\mathcal{V}^{\prime}$-category for a suitable $\mathcal{V}^{\prime}$, and that the only question is the representability of $B \mapsto[\mathcal{K}, \mathcal{V}](F, \mathcal{B}(B, G-)): \mathcal{B}^{\text {op }} \longrightarrow \mathcal{V}^{\prime}$ by an object $\{F, G\}$ of $\mathcal{B}$.

However even more is true: it does not matter in these circumstances whether we perceive the base category as $\mathcal{V}$ or as $\mathcal{V}^{\prime}$ - in the latter case seeing the indexing type as $y F: \mathcal{K} \longrightarrow \mathcal{V} \longrightarrow \mathcal{V}^{\prime}$; for if $\{,\}^{\prime}$ denotes the indexed limit of a $\mathcal{V}^{\prime}$-functor, we have

$$
\{y F, G\}^{\prime} \cong\{F, G\}
$$

either existing if the other does. (For once we are using $y$ loosely to denote both the ordinary functor $\mathcal{V}_{0} \longrightarrow \mathcal{V}_{0}^{\prime}$ and the $\mathcal{V}^{\prime}$-functor $\mathcal{V} \longrightarrow \mathcal{V}^{\prime}$.) The point is that $\left[\mathcal{K}, \mathcal{V}^{\prime}\right](y F, y \mathcal{B}(B, G-))$, as the end in $\mathcal{V}^{\prime}$ of $[F K, \mathcal{B}(B, G K)]^{\prime}$, is by (3.81) the end in $\mathcal{V}^{\prime}$ (and hence also in $\mathcal{V}$ if it exists there) of $[F K, \mathcal{B}(B, G K)]$. Of course it is equally true for colimits that $(y F) \star^{\prime} G \cong F \star G$, for these are just limits in $\mathcal{B}^{\text {op }}$.

\subsection{The existence of a limit- and colimit-preserving universe-enlargement $\mathcal{V} \subset \mathcal{V}^{\prime}$}

On the other hand, $y: \mathcal{V}_{0} \longrightarrow \mathcal{V}_{0}^{\prime}$ does not preserve colimits; and we must ask how far this renders our formulae ambiguous under such a change of universe.

The first place where we used colimits in $\mathcal{V}_{0}$ was in the construction in 2.5 of the free $\mathcal{V}$-category $\mathcal{L}_{\mathcal{V}}$ on the locally-small ordinary category $\mathcal{L}$. This, seen as a $\mathcal{V}^{\prime}$-category, is not the same as the free $\mathcal{V}^{\prime}$-category $\mathcal{L}_{\mathcal{V}^{\prime}}$ on $\mathcal{L}$. We applied this notion in 3.8 to exhibit a conical $\operatorname{limit} \lim T$, where $T: \mathcal{L} \longrightarrow \mathcal{B}_{0}$, as an indexed limit $\{F, G\}$, with $\mathcal{L}_{\mathcal{V}}$ as the domain of $F$ and $G$. When $\mathcal{L}$ is not Set-locally-small, $\mathcal{L}_{\mathcal{V}}$ does not in general exist, but $\mathcal{L}_{\mathcal{V}^{\prime}}$ does so for a suitable $\mathcal{V}^{\prime}$; and this enables us to define $\lim T$ as the appropriate $\left\{F^{\prime}, G^{\prime}\right\}^{\prime}$, where $F^{\prime}$ and $G^{\prime}$ have domain $\mathcal{L}_{\mathcal{V}^{\prime}}$ and $F^{\prime}$ has codomain $\mathcal{V}^{\prime}$. The question arises whether the two definitions of $\lim T$ agree when $\mathcal{L}$ is locally small. The characterization (3.51) shows at once that they do: for the right side of (3.51) is an ordinary limit in $\mathcal{V}_{0}$ and hence in $\mathcal{V}_{0}^{\prime}$.

The only other place where we have used colimits in $\mathcal{V}_{0}$ (except in the negative considerations of 3.9) is in the formula (3.22) expressing the "continuity" of a limit in its index - this meaning more precisely that colimits in $[\mathcal{A}, \mathcal{V}]$ are sent to limits in $\mathcal{B}$. Note that the $\mathcal{V}^{\prime}$-functor $y: \mathcal{V} \longrightarrow \mathcal{V}^{\prime}$, although it preserves small tensor products by (3.77), 
fails to preserve small conical colimits (since by (3.53) these are the ordinary colimits in $\mathcal{V}_{0}$ and $\mathcal{V}_{0}^{\prime}$ ), and hence fails to preserve small indexed colimits; whence the same is true of $[\mathcal{A}, \mathcal{V}] \longrightarrow\left[\mathcal{A}, \mathcal{V}^{\prime}\right]$

The argument given for (3.22) implicitly presupposes the existence of $[\mathcal{A}, \mathcal{V}]$ as a $\mathcal{V}$ category in which $F \star G$ is to live. However the result remains literally true even if $\left[\mathcal{K}^{\mathrm{op}}, \mathcal{V}\right]$ and $[\mathcal{A}, \mathcal{V}]$ exist only as $\mathcal{V}^{\prime}$-categories, provided that we interpret the existence of $F \star G$ as meaning that we have an isomorphism $[\mathcal{A}, \mathcal{V}](F \star G, H) \cong\left[\mathcal{K}^{\text {op }}, \mathcal{V}^{\prime}\right](F,[\mathcal{A}, \mathcal{V}](G-, H))$ in $\mathcal{V}^{\prime}$. For then we have

$$
\begin{aligned}
\mathcal{B}(B,\{F,\{G-, T\}\}) & \cong\left[\mathcal{K}^{\mathrm{op}}, \mathcal{V}\right](F, \mathcal{B}(B,\{G-, T\})) \\
& \cong\left[\mathcal{K}^{\mathrm{op}}, \mathcal{V}\right](F,[\mathcal{A}, \mathcal{V}]((G-) ?, \mathcal{B}(B, T ?))) \\
& \cong[\mathcal{A}, \mathcal{V}]((F \star G) ?, \mathcal{B}(B, T ?)) \\
& \cong \mathcal{B}(B,\{F \star G, T\})
\end{aligned}
$$

We also have, however, the $\mathcal{V}^{\prime}$-analogue of (3.22); this is a different true statement. For now $F$ is replaced by the composite $F^{\prime}=y F: \mathcal{K}^{\mathrm{op}} \longrightarrow \mathcal{V} \longrightarrow \mathcal{V}^{\prime}$, and $G$ by the composite $G^{\prime}: \mathcal{K} \longrightarrow[\mathcal{A}, \mathcal{V}] \longrightarrow\left[\mathcal{A}, \mathcal{V}^{\prime}\right]$; so that $G^{\prime} K$ is $y(G K)$. The right side of (3.22) in fact remains unchanged, by (3.84); but its new left side is $\left\{F^{\prime} \star^{\prime} G^{\prime}, T\right\}^{\prime}$, while its old left side, by two applications of $(3.84)$, is $\{F \star G, T\} \cong\{y(F \star G), T\}^{\prime} \cong\left\{y F \star^{\prime} G, T\right\}^{\prime}$; and $y F \star^{\prime} G=F^{\prime} \star^{\prime} G$ differs from $F^{\prime} \star^{\prime} G^{\prime}$ because $[\mathcal{A}, \mathcal{V}] \longrightarrow\left[\mathcal{A}, \mathcal{V}^{\prime}\right]$ does not preserve colimits.

Thus, in a sense, the ambiguities that arise from the failure of $y: \mathcal{V} \longrightarrow \mathcal{V}^{\prime}$ to preserve colimits are of but minor importance. Still, the notation would be that much more flexible if by modifying $\mathcal{V}^{\prime}$ we could remove them; and more adapted to universal algebra, where colimits in $\mathcal{V}$ do play a central role. Indeed, in the concrete examples of 2.6 , where $\mathcal{V}_{0}^{\prime}$ is not $\left[\mathcal{V}_{0}^{\text {op }}\right.$, Set $\left.^{\prime}\right]$, but consists - in the case $\mathcal{V}=$ Cat for example - of the category objects in Set $^{\prime}$, the inclusion $\mathcal{V}_{0} \longrightarrow \mathcal{V}_{0}^{\prime}$ does preserve all limits and all colimits that exist in $\mathcal{V}_{0}$. We now show that we can do nearly as well as this in the general case, replacing $\mathcal{V}_{0}^{\prime}=\left[\mathcal{V}_{0}^{\text {op }}\right.$, Set $\left.^{\prime}\right]$ by a reflective full subcategory $\mathcal{V}_{0}^{\prime \prime}$ containing $\mathcal{V}_{0}$; it is then still the case, by 3.5 , that $\mathcal{V}_{0} \longrightarrow \mathcal{V}_{0}^{\prime \prime}$ preserves all the limits that exist in $\mathcal{V}_{0}$; and we can arrange for it to preserve, if not all colimits that exist in $\mathcal{V}_{0}$, at least those that are $\mathbf{S e t}^{\prime}$-small - and so a fortiori all small (= Set-small) ones.

We just take $\mathcal{V}_{0}^{\prime \prime}$ to be the full subcategory of $\mathcal{V}_{0}^{\prime}=\left[\mathcal{V}_{0}^{\text {op }}, \mathbf{S e t}^{\prime}\right]$ determined by those $F: \mathcal{V}_{0}^{\text {op }} \longrightarrow$ Set $^{\prime}$ which send all the Set $^{\prime}$-small colimits that exist in $\mathcal{V}_{0}$ - these being limits in $\mathcal{V}_{0}^{\text {op }}$ - to limits in $\mathbf{S e t}^{\prime}$. By 3.2, the representables constituting $\mathcal{V}_{0}$ lie in $\mathcal{V}_{0}^{\prime \prime}$. That $\mathcal{V}_{0} \longrightarrow \mathcal{V}_{0}^{\prime \prime}$ preserves the colimits in question is immediate; and that $\mathcal{V}_{0}^{\prime \prime}$ is reflective in $\mathcal{V}_{0}^{\prime}$ follows from Kennison [50] or from Freyd-Kelly [30]; see also 6.3 below. Let the reflexion be $R: \mathcal{V}_{0}^{\prime} \longrightarrow \mathcal{V}_{0}^{\prime \prime}$.

The first thing to observe is that

$$
[G, H]^{\prime} \in \mathcal{V}_{0}^{\prime \prime} \text { if } G \in \mathcal{V}_{0}^{\prime} \text { and } H \in \mathcal{V}_{0}^{\prime \prime}
$$

For $[G, H]^{\prime}=\int_{Y}[G Y, H(-\otimes Y)]$ by $(3.80)$; but $-\otimes Y$ preserves all colimits, and in particular those in question; $H$ sends these by hypothesis to limits in Set'; the representable $[G Y,-]$ preserves all limits; and so by (3.64) does $\int_{Y}$. 
By the results of Day [14], this is enough to ensure that $\mathcal{V}_{0}^{\prime \prime}$ has a symmetric monoidal closed structure $\mathcal{V}^{\prime \prime}=\left(\mathcal{V}_{0}^{\prime \prime}, \otimes^{\prime \prime}, I^{\prime},[,]^{\prime}\right)$, where

$$
F \otimes^{\prime \prime} G=R\left(F \otimes^{\prime} G\right) .
$$

This will give all that we want; the symmetric monoidal closed structure of $\mathcal{V}^{\prime \prime}$ will extend that of $\mathcal{V}$ by (3.81), (3.83), and (3.77) taken with the fact that $\mathcal{V}_{0} \subset \mathcal{V}_{0}^{\prime \prime}$.

An adaptation of Day's argument to the present case is as follows; in fact we need (3.85) only for $G, H \in \mathcal{V}_{0}^{\prime \prime}$. Then for $F, G, H \in \mathcal{V}_{0}^{\prime \prime}$ we have from $(3.86)$ that $\mathcal{V}_{0}^{\prime \prime}\left(F \otimes^{\prime \prime} G, H\right) \cong$ $\mathcal{V}_{0}^{\prime}\left(F \otimes^{\prime} G, H\right) \cong \mathcal{V}_{0}^{\prime}\left(F,[G, H]^{\prime}\right)$, which by $(3.85)$ is $\mathcal{V}_{0}^{\prime \prime}\left(F,[G, H]^{\prime}\right)$. Thus $-\otimes^{\prime \prime} G \dashv$ $[G,-]^{\prime}: \mathcal{V}_{0}^{\prime \prime} \longrightarrow \mathcal{V}_{0}^{\prime \prime}$.

There is an evident symmetry $F \otimes^{\prime \prime} G \cong G \otimes^{\prime \prime} F$; moreover $I^{\prime} \otimes^{\prime \prime} G=R\left(I^{\prime} \otimes^{\prime} G\right) \cong$ $R G=G$ for $G \in \mathcal{V}_{0}^{\prime \prime}$. As for associativity, let $F, G, H \in \mathcal{V}_{0}^{\prime \prime}$. Then for any $K \in \mathcal{V}_{0}^{\prime \prime}$ we have $[H, K]^{\prime} \in \mathcal{V}_{0}^{\prime \prime}$ by (3.85). Thus any map $F \otimes^{\prime} G \longrightarrow[H, K]^{\prime}$ factorizes uniquely through the reflexion $F \otimes^{\prime} G \longrightarrow R\left(F \otimes^{\prime} G\right)=F \otimes^{\prime \prime} G$. It follows that any map $\left(F \otimes^{\prime} G\right) \otimes^{\prime} H \longrightarrow K$ factorizes uniquely through $\left(F \otimes^{\prime} G\right) \otimes^{\prime} H \longrightarrow\left(F \otimes^{\prime \prime} G\right) \otimes^{\prime} H$. Using (3.86) again, we conclude that $\left(F \otimes^{\prime \prime} G\right) \otimes^{\prime \prime} H \cong R\left(\left(F \otimes^{\prime} G\right) \otimes^{\prime} H\right)$. So the associativity isomorphism for $\otimes^{\prime}$ reflects onto one for $\otimes^{\prime \prime}$; and moreover all the coherence conditions reflect too.

Let us change notation now, and write $\mathcal{V}^{\prime}$ for $\mathcal{V}^{\prime \prime}$; so that henceforth $\mathcal{V}^{\prime}$ denotes an extension of $\mathcal{V}$ for which $\mathcal{V} \longrightarrow \mathcal{V}^{\prime}$ preserves all limits that exist in $\mathcal{V}$ and also all colimits that exist and are $\mathbf{S e t}^{\prime}$-small. In future all functor categories too big to exist as $\mathcal{V}$ categories are to be interpreted as $\mathcal{V}^{\prime}$-categories for a suitable such $\mathcal{V}^{\prime}$; and similarly for limits and colimits in $\mathcal{V}$ or in some $[\mathcal{A}, \mathcal{V}]$. 


\section{Chapter 4}

\section{Kan extensions}

4.1 The definition of Kan extensions; their expressibility by limits and colimits

Recall from (3.38) and (3.39) that a $\mathcal{V}$-functor $K: \mathcal{A} \longrightarrow \mathcal{C}$ induces $\widehat{K}: \mathcal{C}^{\text {op }} \longrightarrow[\mathcal{A}, \mathcal{V}]$ and $\widetilde{K}: \mathcal{C} \longrightarrow\left[\mathcal{A}^{\text {op }}, \mathcal{V}\right]$, given by $\widehat{K} C=\mathcal{C}(C, K-)$ and $\widetilde{K} C=\mathcal{C}(K-, C)$. Note that, for $F: \mathcal{C} \longrightarrow \mathcal{V}$ and $H: \mathcal{C}^{\mathrm{op}} \longrightarrow \mathcal{V}$, we have

$$
F \star \widehat{K} \cong F K, \quad H \star \widetilde{K} \cong H K^{\mathrm{op}} ;
$$

since by $(3.16)$ we have $(F \star \widehat{K}) A=F ? \star \mathcal{C}(?, K A)$, which by the symmetry (3.9) and the Yoneda isomorphism (3.10) is $F K A$.

Given $\mathcal{V}$-functors and a $\mathcal{V}$-natural transformation

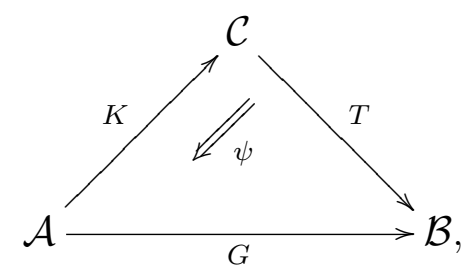

we get for each $F: \mathcal{C} \longrightarrow \mathcal{V}$ a map

$$
[\mathcal{C}, \mathcal{V}](F, \mathcal{B}(B, T-)) \longrightarrow[\mathcal{A}, \mathcal{V}](F K, \mathcal{B}(B, T K-)) \longrightarrow[\mathcal{A}, \mathcal{V}](F K, \mathcal{B}(B, G-)),
$$

where the first arrow is $[K, 1]$ and the second is $[\mathcal{A}, \mathcal{V}](1, \mathcal{B}(1, \psi-))$. Hence by $(3.1)$ we get an induced map

$$
(K, \psi)_{*}:\{F, T\} \rightarrow\{F K, G\}
$$

whenever these limits exist. When $\mathcal{A}=\mathcal{C}$ and $K=1$, this is just

$$
\{1, \psi\}:\{F, T\} \longrightarrow\{F, G\} ;
$$

we saw in (3.11) that $\{$,$\} is a \mathcal{V}$-functor in so far as it exists. When $G=T K$ and $\psi=1$, we may write (4.4) as $K_{*}:\{F, T\} \longrightarrow\{F K, T K\}$. The general (4.4) is the composite

$$
\{F, T\} \underset{K_{*}}{\longrightarrow}\{F K, T K\} \underset{\{1, \psi\}}{\longrightarrow}\{F K, G\}
$$


if all these limits exist; but the outer ones may well exist when the central one does not.

Theorem 4.6 For a diagram (4.2), the following are equivalent:

(i) For each $F: \mathcal{C} \longrightarrow \mathcal{V},(K, \psi)_{*}:\{F, T\} \longrightarrow\{F K, G\}$ is an isomorphism, either limit existing if the other does.

(ii) For each $C \in \mathcal{C}$, the limit $\{\mathcal{C}(C, K-), G$,$\} is given by T C$, with as counit

$$
\mathcal{C}(C, K-) \underset{T}{\longrightarrow} \mathcal{B}(T C, T K-) \underset{\mathcal{B}(1, \psi-)}{\longrightarrow} \mathcal{B}(T C, G-) .
$$

(iii) For each $B \in \mathcal{B}$ and $C \in \mathcal{C}$, the map

$$
\mathcal{B}(B, T C) \longrightarrow[\mathcal{A}, \mathcal{V}](\mathcal{C}(C, K-), \mathcal{B}(B, G-))
$$

induced by (4.7) is an isomorphism.

Proof. (i) gives (ii) on taking $F=\mathcal{C}(C,-)$ and using the Yoneda isomorphism (3.10); (ii) expresses (iii) by the definition (3.1); (iii) asserts by Yoneda that (4.3) is an isomorphism in the case $F=\mathcal{C}(C,-)$, whence (4.3) is an isomorphism for all $F$ by (3.17), both sides of (4.3) sending pointwise colimits in the variable $F$ to limits in $\mathcal{V}$; which then gives (i) by definition.

When these conditions are satisfied, it follows from (ii) that $T$ and $\psi$ are uniquely determined to within isomorphism by $K$ and $G$; we say that the diagram (4.2) (or the map $\psi$ therein) exhibits $T$ as the right Kan extension of $G$ along $K$, and we write $T=\operatorname{Ran}_{K} G$, calling $\psi$ the counit of this right Kan extension. Thus (ii) gives the formula

$$
\left(\operatorname{Ran}_{K} G\right) C=\{\mathcal{C}(C, K-), G\}=\{\widehat{K} C, G\},
$$

and $\operatorname{Ran}_{K} G$ exists, by definition, when the limit on the right exists for each $C \in \mathcal{C}$ : so that we may also write

$$
\operatorname{Ran}_{K} G=\{\widehat{K}-, G\} .
$$

(We may sometimes loosely express the existence of $\{\widehat{K} C, G\}$ for a particular $C$ by saying that $\left(\operatorname{Ran}_{K} G\right) C$ exists. $)$ In terms of the counit $\mu_{C}: \mathcal{C}(C, K-) \rightarrow \mathcal{B}\left(\left(\operatorname{Ran}_{K} G\right) C, G-\right)$ of the limit (4.9), the counit $\psi$ of the right Kan extension is, by (4.7) and Yoneda in the form (1.48),

$$
\psi_{A}=\mu_{K A, A}\left(1_{K A}\right):\left(\operatorname{Ran}_{K} G\right) K A \longrightarrow G A .
$$

The equivalent defining property (i) of Theorem 4.6 may be recorded as

$$
\left\{F, \operatorname{Ran}_{K} G\right\} \cong\{F K, G\} \quad \text { for all } F: \mathcal{C} \longrightarrow \mathcal{V} ;
$$

this may also be seen as coming directly from (4.10), (3.22), and (4.1) - which gives an alternative proof that (iii) implies (i) above. Clearly

$\operatorname{Ran}_{K} G$, where $G: \mathcal{A} \longrightarrow \mathcal{B}$, exists if $\mathcal{A}$ is small and $\mathcal{B}$ is complete.

If $(4.2)$ is a right Kan extension, a functor $P: \mathcal{B} \rightarrow \mathcal{D}$ is said to preserve this right Kan extension if $P \psi$ exhibits $P T$ as $\operatorname{Ran}_{K} P G$. Obviously:

Proposition 4.14 $P$ preserves the right Kan extension $\operatorname{Ran}_{K} G$ if and only if $P$ preserves the limit $\{\widehat{K} C, G\}$ for all $C$. 
In particular, therefore, a right adjoint preserves any right Kan extension that exists; and the representables $\mathcal{B}(B,-): \mathcal{B} \longrightarrow \mathcal{V}$ not only preserve $\operatorname{Ran}_{K} G$ but, in their totality, detect it (which by (3.7) and (4.9) is a re-statement of Theorem 4.6(iii)).

For the dual notion we observe that a diagram

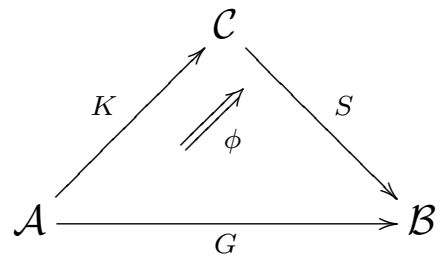

induces, for any $H: \mathcal{C}^{\text {op }} \longrightarrow \mathcal{V}$, a map

$$
(K, \phi)^{*}: H K^{\mathrm{op}} \star G \longrightarrow H \star S ;
$$

we say that $\phi$ exhibits $S$ as the left Kan extension $\operatorname{Lan}_{K} G$ of $G$ along $K$ if (4.16) is an isomorphism for each $H$ whenever either side exists; equivalently, if $\phi$ induces an isomorphism of $S$ with the functor

$$
\operatorname{Lan}_{K} G=(\widetilde{K}-) \star G
$$

which exists when the colimit

$$
\left(\operatorname{Lan}_{K} G\right) C=\widetilde{K} C \star G=\mathcal{C}(K-, C) \star G
$$

does so for each $C$ : as it surely does if $\mathcal{A}$ is small and $\mathcal{B}$ is cocomplete. Then (4.16) becomes

$$
H \star \operatorname{Lan}_{K} G \cong H K^{\mathrm{op}} \star G \quad \text { for all } H: \mathcal{C}^{\mathrm{op}} \longrightarrow \mathcal{V} .
$$

Any left adjoint $Q: \mathcal{B} \longrightarrow \mathcal{D}$ preserves the left Kan extension $\operatorname{Lan}_{K} G$; and a representable $\mathcal{B}(-, B): \mathcal{B}^{\text {op }} \longrightarrow \mathcal{V}$ turns it into a right Kan extension in $\mathcal{V}$ - giving

$$
\mathcal{B}\left(\left(\operatorname{Lan}_{K} G\right) C, B\right) \cong\left[\mathcal{A}^{\mathrm{op}}, \mathcal{V}\right](\mathcal{C}(K-, C), \mathcal{B}(G-, B))
$$

as a further characterization of $\operatorname{Lan}_{K} G$. Since left Kan extensions occur more than right ones in our applications below, we shall give our further results in terms of these; of course the two notions are precisely dual, ( ) op turning a left Kan extension (4.15) into a right one. For the genesis of these ideas, see Kan [38].

\subsection{Elementary properties and examples}

We continue with a collection of elementary results, formulae, and examples.

The accepted term "extension" is somewhat misleading here, for in the diagram

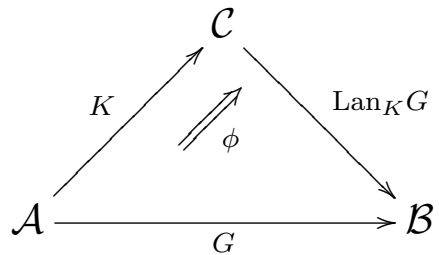


it is not in general the case that $\left(\operatorname{Lan}_{K} G\right) K \cong G$, as (4.34) below shows. The fact is that $\widetilde{K} K: \mathcal{A} \longrightarrow\left[\mathcal{A}^{\text {op }}, \mathcal{V}\right]$ sends $A$ to $\widetilde{K} K A=\mathcal{C}(K-, K A)$, and we have a canonical map $K_{-A}: \mathcal{A}(-, A) \longrightarrow \mathcal{C}(K-, K A)$, which is the $A$-component of a canonical map $\kappa: Y \longrightarrow \widetilde{K} K: \mathcal{A} \longrightarrow\left[\mathcal{A}^{\text {op }}, \mathcal{V}\right]$ where $Y$ is the Yoneda embedding: and clearly $\kappa: Y \longrightarrow \widetilde{K} K$ is an isomorphism if and only if $K$ is fully faithful.

We now have:

Proposition $4.23 \phi: G \longrightarrow\left(\operatorname{Lan}_{K} G\right) K$ is an isomorphism if $K$ is fully faithful; while conversely $K$ is fully faithful if $\phi$ is an isomorphism for all $\mathcal{B}, G$ with $\operatorname{Lan}_{K} G$ existing.

Proof. $\left(\operatorname{Lan}_{K} G\right) K=(\widetilde{K} K-) \star G$ by $(4.17)$, while $G \cong(Y-) \star G$ by the Yoneda isomorphism (3.10); clearly $\phi: G \longrightarrow\left(\operatorname{Lan}_{K} G\right) K$ is $(\kappa-) \star G$; hence it is by (4.22) an isomorphism if $K$ is fully faithful. For the converse take $\mathcal{B}=\mathcal{V}$ and $G=\mathcal{A}(A,-)$; then by (3.9) and (3.10) the isomorphism $\phi_{A}=(\kappa-) \star \mathcal{A}(A,-)$ is $\kappa_{A}$, so that $\kappa$ is an isomorphism and $K$ is fully faithful.

When $\mathcal{B}$ has cotensor products [resp. tensor products] the formulae (4.10) and (4.17) have by (3.69) and (3.70) the more explicit forms

$$
\begin{aligned}
& \operatorname{Ran}_{K} G \cong \int_{A} \mathcal{C}(-, K A) \pitchfork G A \\
& \operatorname{Lan}_{K} G \cong \int^{A} \mathcal{C}(K A,-) \otimes G A
\end{aligned}
$$

either side existing if the other does.

In the classical case $\mathcal{V}=$ Set we can use (3.33) and (3.34) to express the Kan extensions by conical limits and colimits. Observe that el $\mathcal{C}(C, K-)$, where $\mathcal{C}(C, K-): \mathcal{A} \longrightarrow$ Set, is the comma-category $C / K$; an object is $(A \in \mathcal{A}, f: C \longrightarrow K A)$, while a map $(A, f) \longrightarrow\left(A^{\prime}, f^{\prime}\right)$ is some $g: A \longrightarrow A^{\prime}$ with $K g \cdot f=f^{\prime}$. Similarly $\mathcal{C}(K-, C): \mathcal{A}^{\text {op }} \longrightarrow$ Set has el $\mathcal{C}(K-, C)=(K / C)^{\text {op }}$. Hence (3.33) and (3.34) give

$$
\begin{array}{cl}
\left(\operatorname{Ran}_{K} G\right) C \cong \lim (C / K \underset{d}{\longrightarrow} \mathcal{A} \underset{G}{\longrightarrow} \mathcal{B}) \quad \text { for } \mathcal{V}=\text { Set } \\
\left(\operatorname{Lan}_{K} G\right) C \cong \operatorname{colim}(K / C \underset{d}{\longrightarrow} \mathcal{A} \underset{G}{\longrightarrow} \mathcal{B}) \quad \text { for } \mathcal{V}=\text { Set } ;
\end{array}
$$

again either side existing if the other does.

Clearly (4.18) simplifies if $K: \mathcal{A} \longrightarrow \mathcal{C}$ has a right adjoint $L$, for then $\left(\operatorname{Lan}_{K} G\right) C=$ $\mathcal{C}(K-, C) \star G \cong \mathcal{A}(-, L C) \star G$, which is $G L C$ by the Yoneda isomorphism (3.10). Thus

$$
\operatorname{Lan}_{K} G \cong G L \quad \text { if } K \dashv L .
$$

Note that obviously

$$
\text { Any functor } Q: \mathcal{B} \longrightarrow \mathcal{D} \text { preserves the } \operatorname{Lan}_{K} G \text { of }(4.28) \text {. }
$$

We pass to some particular examples. Let $G: \mathcal{A} \longrightarrow \mathcal{B}$, and let $Y: \mathcal{A} \longrightarrow\left[\mathcal{A}^{\text {op }}, \mathcal{V}\right]$ be the Yoneda embedding. Since $\tilde{Y} F=\left[\mathcal{A}^{\mathrm{op}}, \mathcal{V}\right](Y-, F)$ is isomorphic to $F$ by the Yoneda isomorphism (2.31), we have

$$
\widetilde{Y} \cong 1:\left[\mathcal{A}^{\mathrm{op}}, \mathcal{V}\right] \longrightarrow\left[\mathcal{A}^{\mathrm{op}}, \mathcal{V}\right]
$$


Hence (4.17) gives

$$
\operatorname{Lan}_{Y} G \cong-\star G:\left[\mathcal{A}^{\mathrm{op}}, \mathcal{V}\right] \longrightarrow \mathcal{B}
$$

On the other hand, (4.17) also gives $\operatorname{Lan}_{G} Y=(\widetilde{G}-\star Y)$, so that by the Yoneda isomorphism (3.17), we have

$$
\operatorname{Lan}_{G} Y \cong \widetilde{G}: \mathcal{B} \longrightarrow\left[\mathcal{A}^{\text {op }}, \mathcal{V}\right]
$$

We cannot quite say that (4.31) exhibits any colimit $F \star G$ as the value $\left(\operatorname{Lan}_{Y} G\right) F$ of a left Kan extension; for the left Kan extension $\operatorname{Lan}_{Y} G$ may not exist, even though $F \star G$ exists for a particular $F$. Yet we can always exhibit a colimit $F \star G$ as the value of a Kan extension. Identify $\mathcal{A}$ with its full image under $Y: \mathcal{A} \longrightarrow\left[\mathcal{A}^{\mathrm{op}}, \mathcal{V}\right]$, and take for $\mathcal{C}$ the full subcategory of $\left[\mathcal{A}^{\mathrm{op}}, \mathcal{V}\right]$ determined by all the $A \in \mathcal{A}$ and the given $F: \mathcal{A}^{\mathrm{op}} \longrightarrow \mathcal{V}$; let $K: \mathcal{A} \longrightarrow \mathcal{C}$ be the inclusion. Then $\widetilde{K} K \cong Y$ by (4.22), while $\widetilde{K} F \cong F$ by Yoneda. Thus for any $G: \mathcal{A} \longrightarrow \mathcal{B}$ we have $\left(\operatorname{Lan}_{K} G\right) A \cong G A$ for $A \in \mathcal{A}$ (cf. Proposition 4.23), while $\left(\operatorname{Lan}_{K} G\right) F \cong F \star G$. This gives in particular a converse to (4.13):

Proposition $4.33 \mathcal{B}$ admits all left Kan extensions $\operatorname{Lan}_{K} G$, where $K: \mathcal{A} \longrightarrow \mathcal{C}, G: \mathcal{A} \longrightarrow \mathcal{B}$, and $\mathcal{A}$ is small, if and only if it is cocomplete.

In theory, therefore, Kan extensions could replace indexed limits as the basic "limit" notion; we could use (4.8) to define Kan extensions without explicit mention of indexed limits, merely using ordinary limits in $\mathcal{V}_{0}$ to define the functor categories as in 2.2. In fact, as we see in 4.3 below, we can even define Kan extensions without first introducing functor categories: we could then recover these by defining indexed limits, and in particular ends, in terms of Kan extensions. However we find the order adopted in this account more natural.

A conical limit has a particularly simple expression as a Kan extension. If $\mathcal{V}$ is cartesian closed, for instance, colim $G$ coincides with the left Kan extension

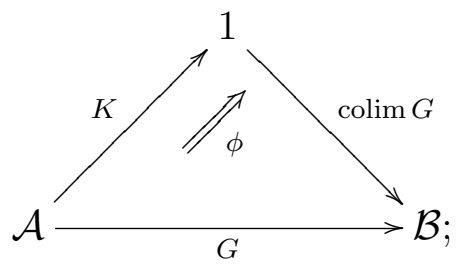

for clearly $\widetilde{K} 0$ is here $\Delta 1: \mathcal{A} \longrightarrow \mathcal{V}$, so that (4.18) gives $\left(\operatorname{Lan}_{K} G\right) 0=\Delta 1 \star G, \cong \operatorname{colim} G$ by (3.57). This illustrates our assertion preceding Proposition 4.23, that $\phi$ is not in general an isomorphism. (Note that (4.34) follows alternatively when $\mathcal{V}=$ Set from (4.27), $d$ then being an equivalence: but the argument we have used here generalizes to conical limits for any $\mathcal{V}$.)

For the purposes of a counter-example we shall use below, we look at a generalization of (4.34). Consider, in the case $\mathcal{V}=$ Set, the left Kan extension

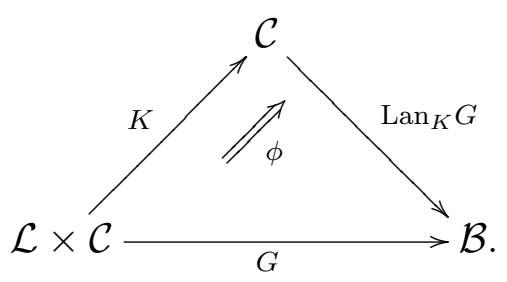


where $K$ is the projection. Here $\widetilde{K} C=\mathcal{C}(K-, C): \mathcal{L}^{\text {op }} \times \mathcal{C}^{\text {op }} \longrightarrow$ Set is clearly $\Delta 1 \times$ $\mathcal{C}(-, C)$; so that $\left(\operatorname{Lan}_{K} G\right) C=(\Delta 1 \times \mathcal{C}(-, C)) \star G$. By the Fubini theorem (3.21), this is $(\Delta 1) ? \star(\mathcal{C}(-, C) \star G(?,-))$, which is $(\Delta 1) ? \star G(?, C)$ by the Yoneda $(3.10)$; whence by $(3.26)$

$$
\left(\operatorname{Lan}_{K} G\right) C \cong \operatorname{colim} G(-, C)
$$

either side existing if the other does. Thus if $G$ corresponds to $T: \mathcal{L} \longrightarrow[\mathcal{C}, \mathcal{B}]$, we conclude that $\operatorname{Lan}_{K} G$ exists in (4.35) exactly when colim $T$ exists pointwise.

\subsection{A universal property of $\operatorname{Lan}_{K} G$; its inadequacy as a definition}

The left Kan extension

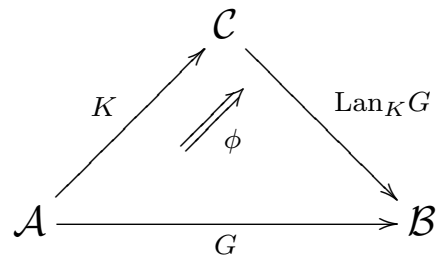

has an important universal property, different from the defining one in its three equivalent forms (4.18), (4.19), (4.20); namely:

Theorem 4.38 For any $S: \mathcal{C} \longrightarrow \mathcal{B}$, we have a $\mathcal{V}$-natural isomorphism

$$
[\mathcal{C}, \mathcal{B}]\left(\operatorname{Lan}_{K} G, S\right) \cong[\mathcal{A}, \mathcal{B}](G, S K)
$$

the unit of this representation being the unit $\phi$ of (4.37). The dual result is

$$
[\mathcal{C}, \mathcal{B}]\left(S, \operatorname{Ran}_{K} G\right) \cong[\mathcal{A}, \mathcal{B}](S K, G)
$$

PROOF.

$$
\begin{aligned}
{[\mathcal{C}, \mathcal{B}]\left(\operatorname{Lan}_{K} G, S\right) } & =\int_{C} \mathcal{B}\left(\left(\operatorname{Lan}_{K} G\right) C, S C\right) & & \text { by }(2.10) \\
& \cong \int_{C}\left[\mathcal{A}^{\mathrm{op}}, \mathcal{V}\right](\mathcal{C}(K-, C), \mathcal{B}(G-, S C)) & & \text { by }(4.20) \\
& \cong \int_{C} \int_{A}[\mathcal{C}(K A, C), \mathcal{B}(G A, S C)] & & \text { by }(2.10) \\
& \cong \int_{A} \int_{C}[\mathcal{C}(K A, C), \mathcal{B}(G A, S C)] & & \text { by }(2.9) \\
& \cong \int_{A} \mathcal{B}(G A, S K A) & & \text { by }(2.33) \\
& \cong[\mathcal{A}, \mathcal{B}](G, S K) & & \text { by }(2.10)
\end{aligned}
$$

Putting $S=\operatorname{Lan}_{K} G$ on the left side and evaluating at 1 to determine the unit, we find this to be $\phi$, using the duals of (4.7) and (4.8). 
Note that the left side of (4.39) exists in $\mathcal{V}$ (and not merely in some extension $\mathcal{V}^{\prime}$ ) if the right side does; in particular

$$
[\mathcal{C}, \mathcal{B}]\left(\operatorname{Lan}_{K} G, S\right) \in \mathcal{V} \text { if the domain } \mathcal{A} \text { of } K \text { is small. }
$$

Applying $V=\mathcal{V}_{0}(I,-): \mathcal{V}_{0} \longrightarrow$ Set to $(4.39)$, we get a bijection of sets

$$
[\mathcal{C}, \mathcal{B}]_{0}\left(\operatorname{Lan}_{K} G, S\right) \cong[\mathcal{A}, \mathcal{B}]_{0}(G, S K)
$$

which in view of (2.16) may be expressed as:

Theorem 4.43 If $\phi: G \longrightarrow L K$ exhibits $L$ as $\operatorname{Lan}_{K} G$, then every $\mathcal{V}$-natural $\alpha: G \longrightarrow S K$ factorizes as

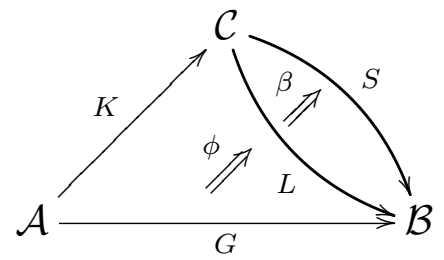

for a unique $\beta: L \longrightarrow S$.

The universal property of Theorem 4.43 can be satisfied by at most one $(L, \phi)$ to within isomorphism; for it is a question of a representation $[\mathcal{C}, \mathcal{B}]_{0}(L, S) \cong[\mathcal{A}, \mathcal{B}]_{0}(G, S K)$. It is moreover a very simple universal property, that makes sense not only in $\mathcal{V}$-CAT but in any 2-category. This has led many authors to use it as the definition of left Kan extension. Let us call an $(L, \phi)$ with this universal property a weak left Kan extension of $G$ along $K$, writing $L=\operatorname{lan}_{K} G$ with a small "l" (and $\operatorname{ran}_{K} G$ for the dual). We now compare this with our definition. Theorem 4.43 asserts that $\operatorname{Lan}_{K} G=\operatorname{lan}_{K} G$ if the former exists. However $L=\operatorname{lan}_{K} G$ need not satisfy

$$
[\mathcal{C}, \mathcal{B}](L, S) \cong[\mathcal{A}, \mathcal{B}](G, S K)
$$

and hence by (4.39) need not be $\operatorname{Lan}_{K} G$. Take $\mathcal{V}=$ Cat, with $\mathcal{A}$ a mere category and $\mathcal{C}=1$. The universal property of $L=\operatorname{lan}_{K} G$ clearly asserts that $L$ is the colimit of $G$ in $\mathcal{B}_{0}$, while (4.45) asserts that $L$ is the colimit of $G$ in $\mathcal{B}$; yet by (3.54) the first of these does not imply the second. (In this particular case, by (4.34), an $L$ satisfying (4.45) is necessarily $\operatorname{Lan}_{K} G$.) Of course $L=\operatorname{lan}_{K} G$ does satisfy (4.45) if $V$ is conservative; it also does so if $\mathcal{B}$ is cotensored, by an argument like that used in 3.8 when comparing conical limits in $\mathcal{B}_{0}$ with those in $\mathcal{B}$. Yet even an $(L, \phi)$ satisfying (4.45) still need not be $\operatorname{Lan}_{K} G$; in other words, the isomorphism (4.39) is strictly weaker than our definition of $\operatorname{Lan}_{K} G$. This is so even in the classical case $\mathcal{V}=$ Set; a counter-example is provided by (4.35), where $\operatorname{Lan}_{K} G$ exists by (4.36) precisely when $T: \mathcal{L} \longrightarrow[\mathcal{C}, \mathcal{B}]$ has a pointwise colimit, while (4.45) merely asserts that $L \cong$ colim $T$, which by 3.3 does not imply pointwiseness.

For such reasons, various authors $[22,60,9]$ who call $\operatorname{lan}_{K} G$ a Kan extension use the name pointwise Kan extension for $\operatorname{Lan}_{K} G$. Our present choice of nomenclature is based on our failure to find a single instance where a weak Kan extension plays any mathematical role whatsoever. (Street in [71] has given a definition of "pointwise Kan extension" in 
any 2-category with finite limits; it agrees with our notion when $\mathcal{V}=$ Set, but is strictly stronger for a general $\mathcal{V}$, and is hence not suited to our context.)

When $\mathcal{B}$ is cotensored and $L=\operatorname{lan}_{K} G$ exists, we have observed that $L$ satisfies (4.45). If in this we now take for $S$ the functor $\mathcal{C}(-, C) \pitchfork B$ for fixed $C \in \mathcal{C}$ and $B \in \mathcal{B}$, we easily get $\mathcal{B}(L C, B) \cong\left[\mathcal{A}^{\text {op }}, \mathcal{V}\right](\mathcal{C}(K-, C), \mathcal{B}(G-, B))$, so that $L=\operatorname{Lan}_{K} G$ by (4.20). Thus for cotensored $\mathcal{B}$ there is no distinction between $\operatorname{lan}_{K} G$ and $\operatorname{Lan}_{K} G$. This is so in particular for $\mathcal{B}=\mathcal{V}$; moreover, since $\mathcal{V}$ is also tensored, $\operatorname{ran}_{K} G$ and $\operatorname{Ran}_{K} G$ too have the same meaning for $\mathcal{B}=\mathcal{V}$. This gives a way of defining $\operatorname{Ran}_{K} G$, for any $\mathcal{B}$, in terms of weak Kan extensions:

Proposition 4.46 The diagram

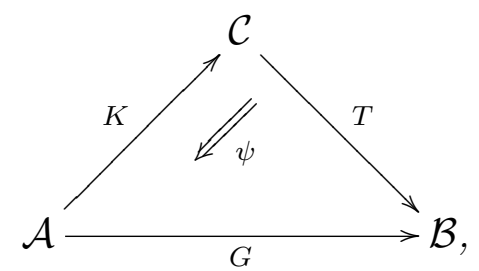

exhibits $T$ as $\operatorname{Ran}_{K} G$ if and only if, for each $B \in \mathcal{B}$, the 2-cell $\mathcal{B}(B, \psi)$ exhibits $\mathcal{B}(B, T-)$ as $\operatorname{ran}_{K} \mathcal{B}(B, G-)$.

Proof. "Only if" is clear since representables preserve $\operatorname{Ran}_{K} G$. For the converse, $\mathcal{B}(B, T-)$ is actually $\operatorname{Ran}_{K} \mathcal{B}(B, G-)$ by the remark preceding the proposition; so that (4.8) is an isomorphism by (4.9) and (3.7), giving $T=\operatorname{Ran}_{K} G$.

It is this way of defining $\operatorname{Ran}_{K} G$, in terms of the universal property of ran, that is envisaged in our remarks following Proposition 4.33.

Before leaving this comparison of Lan with lan, we should perhaps emphasize that, even when $V$ is conservative, $\operatorname{Lan}_{K} G\left(\right.$ or $\left.\operatorname{lan}_{K} G\right)$ has meaning heavily dependent on $\mathcal{V}$. Thus, when $\mathcal{V}=\mathbf{A b}, \operatorname{Lan}_{K} G$ is quite different from $\operatorname{Lan}_{K_{0}} G_{0}$. The former is an additive functor, and satisfies the universal property of Theorem 4.43 among all additive functors; the latter is in general not additive at all.

\subsection{Iterated Kan extensions; Kan adjoints; $\left[\mathcal{A}^{\text {op }}, \mathcal{V}\right]$ as the free cocomple- tion of a small $\mathcal{A}$}

We continue with further elementary properties of Kan extensions which, in one way or another, make use of Theorem 4.38 or Theorem 4.43 .

Theorem 4.47 If $\operatorname{Lan}_{K} G$ exists we have

$$
\operatorname{Lan}_{Z} \operatorname{Lan}_{K} G \cong \operatorname{Lan}_{Z K} G
$$

either side existing if the other does. Then if $\phi$ and $\theta$ are the units of $\operatorname{Lan}_{K} G$ and $\operatorname{Lan}_{Z} \operatorname{Lan}_{K} G$, the unit $\chi$ of $\operatorname{Lan}_{Z K} G$ is the composite natural transformation in the di- 
4.4 Iterated Kan extensions; Kan adjoints; $\left[\mathcal{A}^{\mathrm{op}}, \mathcal{V}\right]$ as the free cocompletion agram

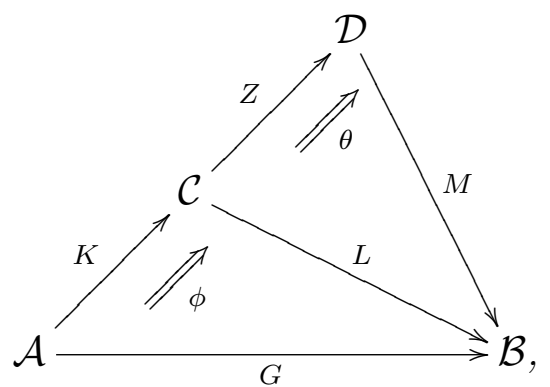

where $L$ denotes $\operatorname{Lan}_{K} G$ and $M$ the common value in (4.48). If $Z$ is fully faithful, $\operatorname{Lan}_{K} G$ exists whenever $\operatorname{Lan}_{Z K} G$ exists, being $\left(\operatorname{Lan}_{Z K} G\right) Z$.

Proof. (4.19) gives $\widetilde{Z} D \star \operatorname{Lan}_{K} G \cong\left(\widetilde{Z} D \cdot K^{\mathrm{op}}\right) \star G$, either side existing if the other does. Since $\widetilde{Z} D \cdot K^{\mathrm{op}}=\mathcal{D}(Z K-, D)$, this is by (4.18) the desired isomorphism (4.48). That the units compose as shown is immediate from Theorem 4.43. The last statement comes from the easy observation that

$$
\widetilde{Z K} Z \cong \widetilde{K} \text { if } Z \text { is fully faithful. }
$$

If $\mathcal{A}$ and $\mathcal{C}$ are small, both $[\mathcal{A}, \mathcal{B}]$ and $[\mathcal{C}, \mathcal{B}]$ exist as $\mathcal{V}$-categories; and any $K: \mathcal{A} \longrightarrow \mathcal{C}$ induces a $\mathcal{V}$-functor $[K, 1]:[\mathcal{C}, \mathcal{B}] \longrightarrow[\mathcal{A}, \mathcal{B}]$ as in $(2.26)$, sending $S$ to $S K$. Then from Theorem 4.38 we get the theorem of Kan adjoints:

Theorem 4.50 Let $K: \mathcal{A} \longrightarrow \mathcal{C}$ be a $\mathcal{V}$-functor with $\mathcal{A}$ and $\mathcal{C}$ small. Then the functor $[K, 1]:[\mathcal{C}, \mathcal{B}] \longrightarrow[\mathcal{A}, \mathcal{B}]$ has the left adjoint $\operatorname{Lan}_{K}$ if $\operatorname{Lan}_{K} G$ exists for all $G: \mathcal{A} \longrightarrow \mathcal{B}$, as it surely does when $\mathcal{B}$ is cocomplete; and $[K, 1]$ has the right adjoint $\operatorname{Ran}_{K}$ if $\operatorname{Ran}_{K} G$ exists for each $G$, as it surely does when $\mathcal{B}$ is complete.

We now consider the sense in which $\left[\mathcal{A}^{\mathrm{op}}, \mathcal{V}\right]$, for a small $\mathcal{A}$, is the free cocompletion of $\mathcal{A}$ (embedded in $\left[\mathcal{A}^{\mathrm{op}}, \mathcal{V}\right]$ by Yoneda).

Theorem 4.51 Let $Y: \mathcal{A} \longrightarrow\left[\mathcal{A}^{\mathrm{op}}, \mathcal{V}\right]$ be the Yoneda embedding, where $\mathcal{A}$ is small, and let $\mathcal{B}$ be arbitrary. Then for any cocontinuous $S:\left[\mathcal{A}^{\mathrm{op}}, \mathcal{V}\right] \longrightarrow \mathcal{B}$ we have

$$
S \cong \operatorname{Lan}_{Y} G \cong-\star G:\left[\mathcal{A}^{\mathrm{op}}, \mathcal{V}\right] \longrightarrow \mathcal{B}
$$

where

$$
G=S Y: \mathcal{A} \longrightarrow \mathcal{B}
$$

Such a cocontinuous $S$ has a right adjoint $T$ given by

$$
T=\widetilde{G}: \mathcal{B} \longrightarrow\left[\mathcal{A}^{\mathrm{op}}, \mathcal{V}\right]
$$

The full subcategory $\operatorname{Cocts}\left[\left[\mathcal{A}^{\mathrm{op}}, \mathcal{V}\right], \mathcal{B}\right]$ of $\left[\left[\mathcal{A}^{\mathrm{op}}, \mathcal{V}\right], \mathcal{B}\right]$ determined by the cocontinuous functors exists as a $\mathcal{V}$-category, and $S \mapsto S Y$ is an equivalence of $\mathcal{V}$-categories

$$
[Y, 1]: \operatorname{Cocts}\left[\left[\mathcal{A}^{\text {op }}, \mathcal{V}\right], \mathcal{B}\right] \simeq[\mathcal{A}, \mathcal{B}]^{\prime}
$$


where $[\mathcal{A}, \mathcal{B}]^{\prime}$ is the full subcategory of $[\mathcal{A}, \mathcal{B}]$ determined by those $G: \mathcal{A} \longrightarrow \mathcal{B}$ for which $\operatorname{Lan}_{Y} G$ exists; the inverse to this equivalence sends $G$ to $\operatorname{Lan}_{Y} G$. In particular, if $\mathcal{B}$ is cocomplete, this is an equivalence

$$
\operatorname{Cocts}\left[\left[\mathcal{A}^{\text {op }}, \mathcal{V}\right], \mathcal{B}\right] \simeq[\mathcal{A}, \mathcal{B}]
$$

Proof. Expressing an arbitrary $F: \mathcal{A}^{\text {op }} \longrightarrow \mathcal{V}$ as a small colimit $F \cong F \star Y$ of representables by (3.17), we see that a cocontinuous $S$ must have $S F \cong F \star S Y$, which is $F \star G$ by (4.53), or $\left(\operatorname{Lan}_{Y} G\right) F$ by $(4.31)$. We then have $\mathcal{B}(S F, B) \cong \mathcal{B}(F \star G, B) \cong$ $\left[\mathcal{A}^{\mathrm{op}}, \mathcal{V}\right](F, \widetilde{G} B)$ by $(3.5)$, so that $S \dashv \widetilde{G}$.

Since the cocontinuous $S$ is $\operatorname{Lan}_{Y} G$, it follows from $(4.41)$ that $\operatorname{Cocts}\left[\left[\mathcal{A}^{\text {op }}, \mathcal{V}\right], \mathcal{B}\right]$ exists as a $\mathcal{V}$-category, and from (4.39) that

$$
\operatorname{Cocts}\left[\left[\mathcal{A}^{\text {op }}, \mathcal{V}\right], \mathcal{B}\right]\left(S, S^{\prime}\right) \cong\left[\left[\mathcal{A}^{\text {op }}, \mathcal{V}\right], \mathcal{B}\right]\left(\operatorname{Lan}_{Y} G, S^{\prime}\right) \cong[\mathcal{A}, \mathcal{B}]\left(G, S^{\prime} Y\right)=[\mathcal{A}, \mathcal{B}]\left(S Y, S^{\prime} Y\right) \text {. }
$$

Thus $[Y, 1]$ in $(4.55)$ is fully faithful. It is moreover essentially surjective, since if $\operatorname{Lan}_{Y} G=$ - $\star G$ exists, it is cocontinuous by $(3.23)$, and $\left(\operatorname{Lan}_{Y} G\right) Y \cong G$ by Proposition 4.23 (or equally by $(3.10))$.

\subsection{Initial diagrams as the left Kan extensions into $\mathcal{V}$; initial and final functors when $\mathcal{V}=$ Set}

As the motivation for our definition of right Kan extension, we chose in 4.1 the property (4.12) which allows us to write a limit $\{F K, G\}$ as $\left\{F, \operatorname{Ran}_{K} G\right\}$. We now examine the corresponding problem of writing a limit $\{H, T K\}$ in the form $\{F, T\}$.

First we need a result resembling (4.12) or (4.19), but where the Kan extension has codomain $\mathcal{V}$ and the other functor need not:

Proposition 4.57 Let $\operatorname{Lan}_{K} H$ exist, where $K: \mathcal{A} \longrightarrow \mathcal{C}$ and $H: \mathcal{A} \longrightarrow \mathcal{V}$; then for any $T: \mathcal{C} \longrightarrow \mathcal{B}$ we have

$$
\left\{\operatorname{Lan}_{K} H, T\right\} \cong\{H, T K\},
$$

either side existing if the other does.

Proof. The formula (3.22) expressing the "continuity of a limit in its index" here gives $\{H ? \star \mathcal{C}(K ?,-), T-\} \cong\{H ?,\{\mathcal{C}(K ?,-), T-\}\}$; but $H ? \star \mathcal{C}(K-, ?)$ is $\operatorname{Lan}_{K} H$ by (4.18) and the symmetry (3.9), while $\{\mathcal{C}(K-, ?), T-\} \cong T K$ by the Yoneda isomorphism (3.10).

Given now a diagram

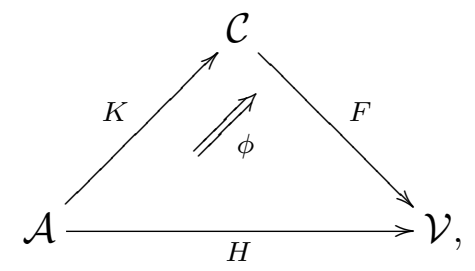


we get for each $T: \mathcal{C} \longrightarrow \mathcal{B}$ a map

$$
[\mathcal{C}, \mathcal{V}](F, \mathcal{B}(B, T-)) \longrightarrow[\mathcal{A}, \mathcal{V}](F K, \mathcal{B}(B, T K-)) \longrightarrow[\mathcal{A}, \mathcal{V}](H, \mathcal{B}(B, T K-))
$$

where the first arrow is $[K, 1]$ and the second is $[\mathcal{A}, \mathcal{V}](\phi, 1)$. This induces by $(3.1)$ a map

$$
(K, \phi)^{\#}:\{F, T\} \longrightarrow\{H, T K\}
$$

whenever these limits exist; it is the composite

$$
\{F, T\} \underset{K_{*}}{\longrightarrow}\{F K, T K\} \underset{\{\phi, 1\}}{\longrightarrow}\{H, T K\}
$$

whenever the middle limit exists as well.

Theorem 4.63 For a diagram (4.59), the following are equivalent:

(i) For every $\mathcal{B}$ and every $T: \mathcal{C} \longrightarrow \mathcal{B}$, the $(K, \phi)^{\#}$ of $(4.61)$ is an isomorphism, either side existing if the other does.

(ii) For every $\mathcal{B}$, every $T: \mathcal{C} \longrightarrow \mathcal{B}$, and every $B \in \mathcal{B}$, the map (4.60) is an isomorphism.

(iii) $\phi$ exhibits $F$ in (4.59) as $\operatorname{Lan}_{K} H$.

Proof. Clearly (ii) implies (i) by the definition (3.1) of the limits, while (ii) itself is, by (3.7), the special case of (i) obtained by replacing $T$ in (4.61) by $\mathcal{B}(B, T-$ ). Since (iii) implies (i) by Proposition 4.57, it remains only to prove that (i) implies (iii).

Let $C \in \mathcal{C}$ and $Z \in \mathcal{V}$. Then applying (i) with $\mathcal{B}=\mathcal{V}$ and with $T=[\mathcal{C}(-, C), Z]$ gives $\{F,[\mathcal{C}(-, C), Z]\} \cong\{H,[\mathcal{C}(K-, C), Z]\}$. Since the internal-hom $[X, Z]$ in $\mathcal{V}$ is the cotensor product $X \pitchfork Z$, the interchange-of-limits formula (3.20) lets us write the last isomorphism as $\{\mathcal{C}(-, C),[F-, Z]\} \cong\{\mathcal{C}(K-, C),[H-, Z]\}$. By the Yoneda isomorphism (3.10), the left side here is $[F C, Z]$; while by $(3.7)$ the right side is $\left[\mathcal{A}^{\text {op }}, \mathcal{V}\right](\mathcal{C}(K-, C), \mathcal{V}(H-, Z))$. It now follows from (4.20) that $F$, with unit $\phi$, is $\operatorname{Lan}_{K} H$.

When these equivalent conditions are satisfied, we say that the pair $(K, \phi)$ in $(4.59)$ is initial. The characterization (i) of Theorem 4.63 is the assertion that the F-indexed limit of any $T$ is the $H$-indexed limit of $T K$, when either exists. The characterization (ii) is a $\mathcal{V}$-enriched version of the assertion that, whether the limits exist or not, the $(F, B)$-cylinders over $T$ coincide with the $(H, B)$-cylinders over $T K$. From the proof, it is immaterial whether we require this only when the domain and codomain of (4.60) exist in $\mathcal{V}$, or when they exist merely in an extension $\mathcal{V}^{\prime}$ as in 3.12.

For the dual notion we keep the diagram (4.59) as it is, but consider in place of $T$ a functor $S: \mathcal{C}^{\text {op }} \longrightarrow \mathcal{B}$. Then (4.60) is replaced by a map

$$
[\mathcal{C}, \mathcal{V}](F, \mathcal{B}(S-, B)) \longrightarrow[\mathcal{A}, \mathcal{V}](H, \mathcal{B}(S K-, B))
$$

and (4.61) by a map

$$
(K, \phi)_{\#}: H \star S K^{\mathrm{op}} \longrightarrow F \star S .
$$


We call $\left(K^{\mathrm{op}}, \phi^{\mathrm{op}}\right)$, in the diagram formed by applying ( ) ${ }^{\mathrm{op}}$ to $(4.59)$, final if $(K, \phi)$ is initial; that is, if (4.64) is always an isomorphism, or if (4.65) is an isomorphism whenever either side exists, or if $F \cong \operatorname{Lan}_{K} H$, or if $F^{\text {op }} \cong \operatorname{Ran}_{K^{\text {op }}} H^{\text {op }}$.

For a cartesian closed $\mathcal{V}$, and in particular when $\mathcal{V}=$ Set, we can consider the special case

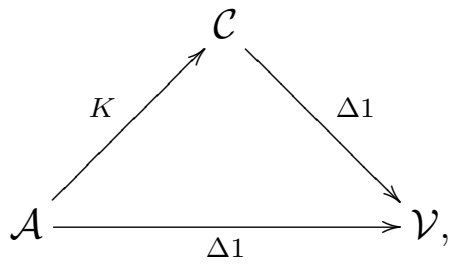

of (4.59); here the unique 2-cell $\phi$ that can be inserted in the diagram is the identity $i d$. We call the functor $K$ initial, and the functor $K^{\mathrm{op}}$ final, if the pair $(K, i d)$ in (4.66) is initial.

Theorem 4.67 For a cartesian closed $\mathcal{V}$ and a $\mathcal{V}$-functor $K: \mathcal{A} \longrightarrow \mathcal{C}$, the following are each equivalent to the initialness of $K$ :

(i) For any $T: \mathcal{C} \longrightarrow \mathcal{B}$ we have $\lim T \cong \lim T K$, either side existing if the other does.

(ii) For such a $T$ and any $B \in \mathcal{B}$ we have $[\mathcal{C}, \mathcal{B}](\Delta B, T) \cong[\mathcal{A}, \mathcal{B}](\Delta B, T K)$.

(iii) The diagram (4.66) exhibits $\Delta 1$ as $\operatorname{Lan}_{K} \Delta 1$.

(iv) For each $C \in \mathcal{C}$ we have $\operatorname{colim} \mathcal{C}(K-, C)=1$.

When $\mathcal{V}=$ Set, (ii) here is just a bijection between the two sets of cones; and (iv) becomes

(v) For each $C$, the comma-category $K / C$ is connected.

Proof. (i) here is by (3.57) the (i) of Theorem 4.63; (ii) is the (ii) of that theorem since, for a cartesian closed $\mathcal{V}$, we have as in 3.9 that $[\mathcal{C}, \mathcal{V}](\Delta 1, \mathcal{B}(B, T-)) \cong[\mathcal{C}, \mathcal{B}](\Delta B, T)$; and (iii) is the (iii) of that theorem. Calculating $\left(\operatorname{Lan}_{K} \Delta 1\right) C$ by (4.18) and using the dual of (3.57) gives (iv); which when $\mathcal{V}=$ Set becomes (v), since (3.35) then gives $\operatorname{colim} \mathcal{C}(K-, C)=\pi(\operatorname{el} \mathcal{C}(K-, C))=\pi(K / C)$.

If $\mathcal{A}=1$ in the above theorem, in the case $\mathcal{V}=\operatorname{Set}, K$ is just an object of $\mathcal{C}$; and the comma-category $K / C$ is just the set $\mathcal{C}(K, C)$ regarded as a discrete category. Hence (v) gives:

Proposition 4.68 When $\mathcal{V}=$ Set, the functor $K: 1 \longrightarrow \mathcal{C}$ is initial [resp. final] exactly when $K$ is an initial [resp. final] object of $\mathcal{C}$.

By (4.28), if $K: \mathcal{A} \longrightarrow \mathcal{C}$ has a right adjoint $L$, we have $\operatorname{Lan}_{K} H \cong H L$ for any $H: \mathcal{A} \longrightarrow \mathcal{V}$. If $\mathcal{V}$ is cartesian closed and $H=\Delta 1$, then $H L=\Delta 1$; so that Theorem 4.67(iii) gives

Proposition 4.69 For cartesian closed $\mathcal{V}$, any left adjoint $K: \mathcal{A} \longrightarrow \mathcal{C}$ is initial. 


\subsection{Filtered categories when $\mathcal{V}=$ Set; the commutativity in Set of fil- tered colimits with finite limits}

Taking $\mathcal{V}=$ Set for this section, we recall for later use the notion of filtered category. There is an interplay between filtered and finite: an ordinary category $\mathcal{P}$ is finite if its set of morphisms (and hence its set of objects) is finite. A category is finitely complete if it admits all (conical) limits indexed by finite categories; and a functor with a finitelycomplete domain is left exact if it preserves all such finite limits: the duals are finitely cocomplete and right exact.

Theorem 4.70 For an ordinary category $\mathcal{C}$, the following are equivalent:

(i) Every functor $T: \mathcal{P} \longrightarrow \mathcal{C}$ with $\mathcal{P}$ finite is the base of some inductive cone.

(ii) $\mathcal{C}$ is not empty; for any $A, B \in \mathcal{C}$ there is some $C \in \mathcal{C}$ and maps $A \longrightarrow C$ and $B \longrightarrow C$; and for any maps $f, g: A \longrightarrow B$ in $\mathcal{C}$ there is some map $h: B \longrightarrow D$ with $h f=h g$.

(iii) For any finite category $\mathcal{P}$ the diagonal $\Delta: \mathcal{C} \longrightarrow[\mathcal{P}, \mathcal{C}]$ has the property that $T / \Delta$ is non-empty for each $T: \mathcal{P} \longrightarrow \mathcal{C}$.

(iv) For any finite category $\mathcal{P}$ the diagonal $\Delta: \mathcal{C} \longrightarrow[\mathcal{P}, \mathcal{C}]$ is final.

ProOF. The properties in (ii) are special cases of (i), and in turn imply (i) by a trivial induction; while (iii) merely re-states (i). It remains to show that these imply (iv), which by Theorem $4.67(\mathrm{v})$ is the requirement that the $T / \Delta$ in (iii) be not merely non-empty but in fact connected. Given objects $\alpha$ and $\beta$ of $T / \Delta-$ that is, cones $\left(\alpha_{P}: T P \longrightarrow A\right)$ and $\left(\beta_{P}: T P \longrightarrow B\right)$ - we can find by (ii) maps $f: A \longrightarrow C$ and $g: B \longrightarrow C$ such that $f \alpha_{P}=g \beta_{P}$ for each $P \in \mathcal{P}$. This common value is now a cone $\left(\gamma_{P}: T P \longrightarrow C\right)$; and we have maps $f: \alpha \longrightarrow \gamma$ and $g: \beta \longrightarrow \gamma$ in $T / \Delta$.

A category $\mathcal{C}$ satisfying these equivalent conditions is said to be filtered. It is clear that a filtered category is connected, and also that $[\mathcal{Q}, \mathcal{C}]$ is filtered if $\mathcal{Q}$ is finite and $\mathcal{C}$ is filtered. Observe that a finitely cocomplete category is filtered, since $T: \mathcal{P} \longrightarrow \mathcal{C}$ is then the base of its colimit-cone; in this case, of course, $\Delta: \mathcal{C} \longrightarrow[\mathcal{P}, \mathcal{C}]$ has a left adjoint, which by Proposition 4.69 is stronger than being final. The initialness condition of Theorem 4.67(v), or rather its dual, simplifies when $\mathcal{C}$ is filtered and $K$ is fully faithful:

Proposition 4.71 If $\mathcal{C}$ is filtered, the fully-faithful $K: \mathcal{A} \longrightarrow \mathcal{C}$ is final if and only if each $C / K$ is non-empty.

Proof. Only the "if" clause needs proof. Consider objects $f: C \longrightarrow K A$ and $g: C \longrightarrow K B$ of $C / K$. Because $\mathcal{C}$ is filtered, there are maps $h: K A \longrightarrow D$ and $k: K B \longrightarrow D$ with $h f=k g$; because $D / K$ is non-empty we may suppose $D$ to be of the form $K E$; because $K$ is fully faithful, $h$ and $k$ have the form $K u$ and $K v$. Thus $f$ and $g$ are connected in $C / K$ by the maps $u: f \longrightarrow h f$ and $v: g \longrightarrow k g=h f$. 
The classical example of this is that where $\mathcal{C}$ is a filtered preordered set (in which case directed is often used for filtered), and $K$ is the inclusion of a subset $\mathcal{A}$. To say that each $C / K$ is non-empty is then to say that each $C \in \mathcal{C}$ is less than or equal to some $A \in \mathcal{A}$. Such a subset $\mathcal{A}$, which we call final, was classically called cofinal.

A filtered colimit is a conical one indexed by a small filtered category. For example, suppose we have in any category a coproduct $A=\sum_{x \in X} A_{x}$. The finite subsets $J$ of $X$, ordered by inclusion, form a filtered ordered set $\mathcal{C}$; and if the finite coproduct $A_{J}=$ $\sum_{x \in J} A_{x}$ exists for each $J \in \mathcal{C}$, it is clear that $A$ is the filtered colimit of the $A_{J}$.

Recall from (3.35) that colim $F$ for $F: \mathcal{C} \longrightarrow$ Set is $\pi(\mathrm{el} F)$.

Theorem 4.72 For $F: \mathcal{C} \longrightarrow$ Set where $\mathcal{C}$ is small and filtered, two objects $(A, x)$ and $(B, y)$ of $\mathrm{el} F$ have the same image in $\pi(\mathrm{el} F)=\operatorname{colim} F$ if and only if there are maps $f: A \longrightarrow C$ and $g: B \longrightarrow C$ with $(F f) x=(F g) y$.

Proof. The relation between $(A, x)$ and $(B, y)$ expressed by the existence of such $f$ and $g$ is reflexive and symmetric, and clearly generates the equivalence relation of being connected. It therefore coincides with this latter if it is transitive; and that it is so follows easily from the filteredness.

If $\mathcal{B}$ is an equational category of algebras whose operations all have finite arity, the conservative forgetful functor $U: \mathcal{B} \longrightarrow$ Set preserves (and therefore reflects) filtered colimits. For if $F: \mathcal{C} \longrightarrow \mathcal{B}$ with $\mathcal{C}$ small and filtered, any finite number of elements of $X=$ colim $U F$ can by the filteredness be represented by elements $\left(C, x_{i}\right) \in \operatorname{el}(U F)$ with a common $C$, so that we can apply an $n$-ary operation $\omega$ to get $\omega\left(x_{1}, \ldots, x_{n}\right) \in U F C$. By Theorem 4.72, the image of this in $X$ is independent of the choice of $C$; thus $X$ becomes $U A$ for an algebra $A$, which is clearly the colimit of $F$.

Theorem 4.73 Filtered colimits commute with finite limits in Set. That is to say, if $F: \mathcal{P} \times \mathcal{C} \longrightarrow$ Set with $\mathcal{P}$ finite and $\mathcal{C}$ small and filtered, the evident canonical map

$$
\rho: \operatorname{colim}_{C} \lim _{P} F(P, C) \longrightarrow \lim _{P} \operatorname{colim}_{C} F(P, C)
$$

is an isomorphism.

Proof. By (3.28) and (3.35), an element of $\lim _{P} \operatorname{colim}_{C} F(P, C)$ is a cone $\left(\alpha_{P}: 1 \longrightarrow \pi(\mathrm{el} F(P,-))\right)$. By the filteredness of $\mathcal{C}$ we can find representatives $\left(C, x_{P} \in F(P, C)\right)$ of the $\alpha_{P}$, with a common $C$. For $f: P \longrightarrow Q$ in $\mathcal{P}$, the objects $\left(C, F(f, C) x_{P}\right)$ and $\left(C, x_{Q}\right)$ represent the same element of $\pi(\mathrm{el} F(Q,-))$, because $\alpha$ is a cone. Since there are but a finite number of maps in $\mathcal{P}$, we can by the filteredness of $\mathcal{C}$ find a $g: C \longrightarrow D$ in $\mathcal{C}$ such that $\left(D, F(f, g) x_{P}\right)=\left(D, F(Q, g) x_{Q}\right)$ for each map $f: P \longrightarrow Q$ in $\mathcal{P}$. Now the $y_{P}=F(P, g) x_{P}$ constitute a cone with vertex 1 over $F(-, D)$, or an element $y$ of $\lim _{P} F(P, D)$. If $z$ is the image of this in $\operatorname{colim}_{C} \lim _{P} F(P, C)$, we clearly have $\rho(z)=\alpha$; thus $\rho$ is surjective.

To see that $\rho$ is injective, suppose that $y \in \lim _{P} F(P, D)$ and $y^{\prime} \in \lim _{P} F\left(P, D^{\prime}\right)$ represent elements of $\operatorname{colim}_{C} \lim _{P} F(P, C)$ with the same image under $\rho$. Then $y_{P} \in$ $F(P, D)$ and $y_{P}^{\prime} \in F\left(P, D^{\prime}\right)$ represent for each $P$ the same element of $\operatorname{colim}_{C} F(P, C)$. As in Theorem 4.72, but using the filteredness to do it simultaneously for each $P \in \mathcal{P}$, we can find $h: D \longrightarrow E$ and $h^{\prime}: D^{\prime} \longrightarrow E$ such that $F(P, h) y_{P}=F\left(P, h^{\prime}\right) y_{P}^{\prime}=w_{P}$ say. Now $w$ 
is a cone in $\lim _{P} F(P, E)$, representing the same object of $\operatorname{colim}_{C} \lim _{P} F(P, C)$ as $y$ and $y^{\prime}$.

By the remark before the theorem, we have the same commutativity if Set is replaced by an equational category of algebras. The theorem can also be stated as:

Proposition 4.74 For small $\mathcal{C}$, the functor colim: $[\mathcal{C}$, Set $] \longrightarrow$ Set is left exact if $\mathcal{C}$ is filtered.

In this form it has a converse, to be given in 5.10 below.

The ideas of this section have the following extension. For a regular cardinal $\alpha$, a category $\mathcal{P}$ is $\alpha$-small if its set of morphisms has cardinal less than $\alpha$; and $\mathcal{C}$ is $\alpha$-filtered if every $T: \mathcal{P} \longrightarrow \mathcal{C}$ with $\alpha$-small $\mathcal{P}$ is the base of some inductive cone. Then $\alpha$-filtered colimits commute with $\alpha$-small limits in Set, by an argument like that for Theorem 4.73 - which is the special case $\alpha=\aleph_{0}$ of this.

\subsection{The factorization of a functor, when $\mathcal{V}=$ Set, into an initial functor and a discrete op-fibration}

In this section, again taking $\mathcal{V}=$ Set, we describe the comprehensive factorization system of Street and Walters [74] on the ordinary category $\mathbf{C a t}_{0}$ of small categories; for its logical significance, see their article.

A functor $J: \mathcal{C} \longrightarrow \mathcal{B}$ is called a discrete op-fibration (or dof for short) if there is a functor $F: \mathcal{B} \longrightarrow$ Set such that $J$ is the composite of an isomorphism $\mathcal{C} \cong$ el $F$ and the canonical projection $d:$ el $F \longrightarrow \mathcal{B}$.

Proposition $4.75 \mathrm{~J}: \mathcal{C} \longrightarrow \mathcal{B}$ is a dof if and only if every map $f: J C \longrightarrow B$ in $\mathcal{B}$ is of the form $f=J g$ for a unique map $g: C \longrightarrow D$ in $\mathcal{C}$.

Proof. "Only if" is immediate; "if" is easy when we define $F: \mathcal{B} \longrightarrow$ Set on objects by $F B=\{D \in \mathcal{C} \mid J D=B\}$ and on maps by the property in the proposition.

It is clear from this proposition that the composite of two dofs is a dof; and it is clear from Theorem 4.67(i) that the composite of two initial functors is initial. Of course an isomorphism in $\mathbf{C a t}_{0}$ is both initial and a dof. We shall show that every map in $\mathbf{C a t}_{0}$ factorizes, essentially uniquely and functorially, into an initial functor followed by a dof.

First observe that, if $F: \mathcal{B} \longrightarrow$ Set gives rise to the dof $d:$ el $F \longrightarrow \mathcal{B}$, to give a functor $T: \mathcal{A} \longrightarrow$ el $F$ is to give a functor $S: \mathcal{A} \longrightarrow \mathcal{B}$ and a cone $\alpha: \Delta 1 \longrightarrow F S$ (or, by (3.28), an element $\alpha \in \lim F S)$. Then $T A=\left(S A, \alpha_{A}\right) \in \operatorname{el} F$.

Given now an arbitrary functor $S: \mathcal{A} \longrightarrow \mathcal{B}$ in $\mathbf{C a t}_{0}$, let $(F, \phi)$ be the left Kan extension

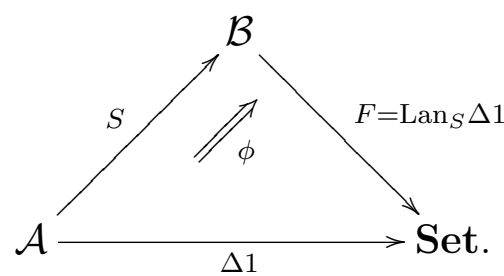


By (4.18), (3.26), and (3.35) we have

$$
F B=\mathcal{B}(S-, B) \star \Delta 1 \cong \operatorname{colim} \mathcal{B}(S-, B) \cong \pi(S / B) .
$$

The functor $S$, together with the cone $\phi: \Delta 1 \longrightarrow F S$, constitutes a functor $T: \mathcal{A} \longrightarrow$ el $F$ with $d T=S$. We shall have factorized $S$ in the desired way if we show that $T$ is initial.

This seems to be best done by a direct calculation using Theorem 4.67(v). (The purported proof in [74] is erroneous; it supposes that, in the situation of our Theorem 4.47, if $X$ and $\theta$ are left Kan extensions, so is $\phi$; that this is false is easily seen by taking $\mathcal{D}=1$ and using (4.34).)

A typical object of el $F$, by $(4.77)$, is $(B,[f])$ where $[f]$ is the connected component of some $f: S A \longrightarrow B$. It is clear that $T: \mathcal{A} \longrightarrow$ el $F$ sends $A$ to $\left(S A,\left[1_{S A}\right]\right)$ and sends $h: A \longrightarrow A^{\prime}$ to $S h$. An object of the comma-category $T /(B,[f])$ is at once seen to be a pair $(A, g: S A \longrightarrow B)$ with $[g]=[f]$. A morphism $(A, g) \longrightarrow\left(A^{\prime}, g^{\prime}\right)$ is an $h: A \longrightarrow A^{\prime}$ for which $g^{\prime} S h=g$. It follows that $T /(B,[f])$ is connected, being isomorphic to the connected component $[f]$ of $S / B$.

To see that this factorization is essentially unique and functorial - that is, that we have a factorization system in the sense of [30] - it remains to show the existence of a unique "diagonal" $R: \mathcal{D} \longrightarrow \mathcal{A}$ in any commutative diagram

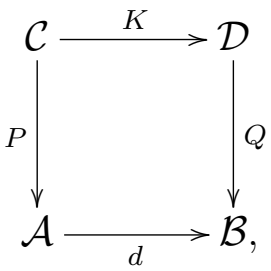

where $K$ is initial and $d$ is a dof. We may as well suppose at once that $\mathcal{A}=$ el $F$ for some $F: \mathcal{B} \longrightarrow$ Set. Then $P$ corresponds as above to a functor $S: \mathcal{C} \longrightarrow \mathcal{B}$ with $d P=S$, and a cone $\alpha: \Delta 1 \longrightarrow F S=F d P=F Q K$. Since $K$ is initial, this cone is by Theorem 4.67(ii) of the form $\alpha=\beta K$ for a unique cone $\beta: 1 \longrightarrow F Q$. Now $Q$ and $\beta$ constitute a functor $R: \mathcal{D} \longrightarrow \mathcal{A}$ with $d R=Q$, and clearly with $R K=P$. It is evident that any $R^{\prime}: \mathcal{D} \longrightarrow \mathcal{A}$ with $d R^{\prime}=Q$ and $R^{\prime} K=P$ must coincide with $R$. This completes the proof.

If we write dof $/ \mathcal{B}$ for the full subcategory of $\mathbf{C a t}_{0} / \mathcal{B}$ determined by those $J: \mathcal{A} \longrightarrow \mathcal{B}$ that are dofs, it is clear that this factorization gives a reflexion $\operatorname{Cat}_{0} / \mathcal{B} \rightarrow \operatorname{dof} / \mathcal{B}$. On the other hand dof $/ \mathcal{B}$ is evidently equivalent to $[\mathcal{B}$, Set $]$. Thus we get an adjunction

$$
\Omega \dashv \Theta:[\mathcal{B}, \text { Set }] \longrightarrow \mathrm{Cat}_{0} / \mathcal{B}
$$

with $\Theta$ fully faithful. Here $\Theta$ sends $F: \mathcal{B} \longrightarrow$ Set to $d:$ el $F \longrightarrow \mathcal{B}$, and may be called the discrete Grothendieck construction; its left adjoint $\Omega$ sends $S$ to the $F$ of (4.77).

While this is special to the case $\mathcal{V}=$ Set, it has generalizations to the important cases $\mathcal{V}=\mathbf{G p d}$ and $\mathcal{V}=\mathbf{C a t}$, the latter being the setting for the original Grothendieck construction [34]. We do not give these here, for they transcend the context of enriched 
category theory as developed in this account, and involve "transformations" more general then the Gpd-natural or Cat-natural ones; we shall treat them in [46].

Note finally that $\Omega \Theta S \cong S$, since $\Theta$ is fully faithful. This means by (4.76) that, for any $F: \mathcal{B} \longrightarrow$ Set, we have a left Kan extension

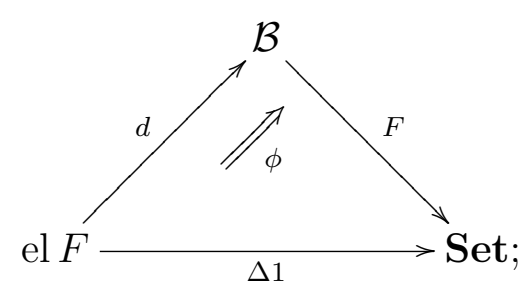

since this asserts by $(4.77)$ that $F B \cong \operatorname{colim} \mathcal{B}(d-, B)$, it is precisely a restatement of $(3.32)$.

\subsection{The general representability and adjoint-functor theorems; the special case of a complete domain-category}

In this section and the next we look at conditions ensuring the representability of a $\mathcal{V}$ functor $F: \mathcal{C} \longrightarrow \mathcal{V}$, especially in the simplest case of a complete $\mathcal{C}$. Since by 1.11 the $\mathcal{V}$-functor $T: \mathcal{C} \longrightarrow \mathcal{B}$ has a left adjoint precisely when each $\mathcal{B}(B, T-)$ is representable, the same considerations yield conditions for a left adjoint to exist.

Since a colimit in $\mathcal{C}$ is itself the representing object for a functor $\mathcal{C} \longrightarrow \mathcal{V}$, the representing object for $F: \mathcal{C} \longrightarrow \mathcal{V}$, if it exists, is in some sense a "generalized colimit". Representing objects are, in fact, very commonly constructed as colimits: consider some examples. First, in the case $\mathcal{V}=$ Set, a representation for $F$ corresponds, as we observed in 1.10, to an initial object in el $F$; that is, to the colimit of the empty diagram in el $F$. Next, in Proposition 3.36, a functor $F: \mathcal{C} \longrightarrow \mathcal{V}$ was in effect given as an iterated limit of representables; and we constructed its representing object as the corresponding iterated colimit of the representing objects for these. Again, the left Kan adjoint Lan $_{K}$ of Theorem 4.50 was constructed via the colimits $\widetilde{K} C \star G$. A free abelian group is constructed as a coproduct of infinite cyclic groups; the tensor product of two abelian groups is constructed as a cokernel of a free abelian group; and the free monoid on a set $X$ is the coproduct $\sum_{n \geq 0} X^{n}$. More generally, numerous constructions of free algebras, free monoids, and free monads, as iterated colimits, under cocompleteness but no completeness hypotheses, are given in [44].

Yet the only general necessary condition that we so far have for the representability of $F$ is expressed in terms of limits: by 3.2, $F$ must preserve whatever limits exist. Hence if $\mathcal{C}$ is complete, $F$ must be continuous (and similarly for a $T: \mathcal{C} \longrightarrow \mathcal{B}$ that is to have a left adjoint). We here consider what must be added to this continuity to ensure representability (or existence of an adjoint). Since the desired representing object is a generalized colimit, this is analogous to the question of what must be added to 
completeness to ensure cocompleteness - or rather, to ensure the existence of a particular colimit.

A small complete Set-category $\mathcal{C}$ is of necessity a preordered set - for if $\mathcal{C}(A, B)$ had at least two elements, $\mathcal{C}\left(A, B^{n}\right)$ would have at least $2^{n}$, making $\mathcal{C}$ large if $B^{n}$ existed for all small cardinals $n$. Hence, by the classical argument on complete lattices, a small complete $\mathcal{C}$ is also cocomplete: the supremum of a subset is the infimum of its set of upper bounds. Not so, if $\mathcal{C}$ is large - even if $\mathcal{C}$ is a preordered set: the ordered set of small ordinals is cocomplete but not complete. What must be added is a condition ensuring that the colimit we need can be manufactured from small limits. Similarly in the case of representability of $F: \mathcal{C} \longrightarrow \mathcal{V}$ : the extra condition needed is a "smallness" one on $F$.

We begin with a general result expressing a representing object as a limit: a large one, unless $\mathcal{C}$ happens to be small.

Theorem $4.80 F: \mathcal{C} \longrightarrow \mathcal{V}$ is representable if and only if the limit $\left\{F, 1_{\mathcal{C}}\right\}$ exists and is preserved by $F$. Then the representing object is $\{F, 1\}$, and the counit $\mu: F \longrightarrow \mathcal{C}(\{F, 1\},-)$ of this limit is an isomorphism giving the representation of $F$.

Proof. One direction is immediate: if $F$ is the representable $\mathcal{C}(D,-)$, then by $(3.10)$ the limit $\{\mathcal{C}(D,-), 1\}$ is $D$, with counit $\mu=1: \mathcal{C}(D,-) \longrightarrow \mathcal{C}(D,-)$; similarly, with appropriate changes, when $F$ is only isomorphic to $\mathcal{C}(D,-)$; and the representable $F$ preserves this limit.

For the other direction we have by hypothesis that

$$
F \underset{\mu}{\longrightarrow} C(\{F, 1\},-) \underset{F}{\longrightarrow}[F\{F, 1\}, F-]
$$

is the counit of the limit $\{F, F\}$. Hence by $(3.4)$ the $(F, I)$-cylinder over $F$ given by the isomorphism $i: F \longrightarrow[I, F-]$ has the form $i=[g, 1] F \mu$ for a unique $g: I \longrightarrow F\{F, 1\}$. Writing $\lambda: \mathcal{C}(\{F, 1\},-) \longrightarrow F$ for the composite $i^{-1}[g, 1] F$, we therefore have $\lambda \mu=1$; and to prove $\mu$ an isomorphism it remains to show that $\mu \lambda: \mathcal{C}(\{F, 1\},-) \longrightarrow \mathcal{C}(\{F, 1\},-)$ is 1 .

But by Yoneda, this map $\mu \lambda$ is $\mathcal{C}(f, 1)$ for a unique $f:\{F, 1\} \rightarrow\{F, 1\}$. Since $\lambda \mu=1$ we have $\mu \lambda \mu=\mu$, or $\mathcal{C}(f, 1) \mu=\mu=\mathcal{C}(1,1) \mu$. However, as the counit of the limit $\{F, 1\}$, the cylinder $\mu$ factorizes through itself as $\mathcal{C}(f, 1) \mu$ for a unique $f$; whence $f=1$ and $\mu \lambda=1$.

There is a corresponding adjunction theorem:

Theorem 4.81 $\mathrm{T}: \mathcal{C} \longrightarrow \mathcal{B}$ has a left adjoint if and only if $\operatorname{Ran}_{T} 1_{\mathcal{C}}$ exists and is preserved by $T$. Then the left adjoint $S$ is $\operatorname{Ran}_{T} 1$, and the counit $S T \longrightarrow 1$ of the Kan extension is the counit of the adjunction.

Proof. If $S \dashv T$ we have $\operatorname{Ran}_{T} 1 \cong S$ by (the dual of) (4.28); that the counit is as described is immediate; and the right adjoint $T$ preserves the right Kan extension.

For the converse, observe that $\left(\operatorname{Ran}_{T} 1\right) B=\{\mathcal{B}(B, T-), 1\}$ by (4.9). This limit is, by hypothesis, preserved by $T$ : hence it is also preserved by $\mathcal{B}(B, T-)$. Thus $\mathcal{B}(B, T-)$ is representable by Theorem 4.80. This being for all $B$, we conclude that $T$ has a left adjoint. 
Combining Theorem 4.63 with Theorem 4.80 gives the following, which replaces the large limit $\{F, 1\}$ by one that may be smaller:

Proposition 4.82 Let $F: \mathcal{C} \longrightarrow \mathcal{V}$ be $\operatorname{Lan}_{K} H$, where $K: \mathcal{A} \longrightarrow \mathcal{C}$ and $H: \mathcal{A} \longrightarrow \mathcal{V}$. Then $F$ is representable if and only if the limit $\{H, K\}$ exists and is preserved by $F$; and the representing object is then $\{H, K\}$.

We consider the possibility of a small $\mathcal{A}$ here:

Proposition 4.83 The following properties of $F: \mathcal{C} \longrightarrow \mathcal{V}$ are equivalent:

(i) F is a small (indexed) colimit of representables.

(ii) $F$ has the form $\operatorname{Lan}_{K} H$ where the domain $\mathcal{A}$ of $K$ is small.

(iii) $F$ is the left Kan extension of its restriction to a small full subcategory of $\mathcal{C}$.

Proof. To say that $F$ is a small colimit of representables is to say that we have a small indexing-type $H: \mathcal{A} \longrightarrow \mathcal{V}$ and a functor $K: \mathcal{A} \longrightarrow \mathcal{C}$ such that, if $Y: \mathcal{C}^{\text {op }} \longrightarrow[\mathcal{C}, \mathcal{V}]$ is the Yoneda embedding $\left([\mathcal{C}, \mathcal{V}]\right.$ being a $\mathcal{V}^{\prime}$-category for some suitable extension $\mathcal{V}^{\prime}$ of $\mathcal{V}$ ), we have $H \star Y K^{\mathrm{op}} \cong F$. Since small colimits in $[\mathcal{C}, \mathcal{V}]$ exist pointwise by 3.3 , this is to say that $H \star \mathcal{C}(K-, C) \cong F C$ for each $C$; and hence by (4.18) and (3.9) to say that $F=\operatorname{Lan}_{K} H$. Thus (i) and (ii) are equivalent. It remains to show that, if we have (ii), we also have its special case (iii).

For this, let $\mathcal{A}^{\prime}$ be the full image of $K: \mathcal{A} \longrightarrow \mathcal{C}$, so that $K$ factorizes as say $W: \mathcal{A} \longrightarrow \mathcal{A}^{\prime}$ followed by the inclusion $Z: \mathcal{A}^{\prime} \longrightarrow \mathcal{C}$. Then $\mathcal{A}^{\prime}$ is still small; and because $Z$ is fully faithful, we have $F \cong \operatorname{Lan}_{Z} F Z$ by Theorem 4.47 .

We shall call a functor $F: \mathcal{C} \longrightarrow \mathcal{V}$ satisfying the equivalent properties of Proposition 4.83 accessible. In particular every representable $\mathcal{C}(D,-): \mathcal{C} \longrightarrow \mathcal{V}$ is accessible; it is in fact $\operatorname{Lan}_{D} I$ where $D: \mathcal{I} \longrightarrow \mathcal{C}$ and $I: \mathcal{I} \longrightarrow \mathcal{V}$. So Proposition 4.82 now gives our desired representability theorem for complete $\mathcal{C}$ :

Theorem 4.84 If $\mathcal{C}$ is complete, $F: \mathcal{C} \longrightarrow \mathcal{V}$ is representable if and only if it is continuous and accessible.

For a general complete $\mathcal{C}$, the representability of $F$ is not implied by its continuity alone, even in the case $\mathcal{V}=$ Set. The classical counter-example takes for $\mathcal{C}$ the category of (small) groups, and for $F: \mathcal{C} \longrightarrow$ Set the functor given by $F C=\prod_{\alpha} \mathcal{C}\left(G_{\alpha}, C\right)$, where $\left\{G_{\alpha}\right\}$ is the large set of all small simple groups.

\subsection{Representability and adjoint-functor theorems when $\mathcal{V}=$ Set}

Although Theorem 4.84 gives a simple general criterion that we shall use below in 5.6, it is often advantageous, in considering the representability of a particular $\mathcal{V}$-functor, to reduce the problem to the more familiar case $\mathcal{V}=$ Set by using: 
Theorem 4.85 Let $\mathcal{C}$ admit cotensor products. Then the $\mathcal{V}$-functor $F: \mathcal{C} \longrightarrow \mathcal{V}$ is representable if and only if the ordinary functor

$$
\mathcal{C}_{0} \underset{F_{0}}{\longrightarrow} \mathcal{V}_{0} \underset{V}{\longrightarrow} \text { Set }
$$

is representable and $F$ preserves cotensor products. Moreover a $\mathcal{V}$-functor $T: \mathcal{C} \longrightarrow \mathcal{B}$ has a left adjoint if and only if the ordinary functor $T_{0}: \mathcal{C}_{0} \longrightarrow \mathcal{B}_{0}$ does so and $T$ preserves cotensor products.

Proof. "Only if" is clear both in the representability case and in the adjunction case - in the former, if $F \cong \mathcal{C}(D,-)$, then $V F_{0} \cong \mathcal{C}_{0}(D,-)$ by $(1.33)$.

For the converse, let $\beta: \mathcal{C}_{0}(D,-) \cong V F_{0}$ be a representation with unit $\eta \in V F D$; then $\eta: I \longrightarrow F D$ corresponds by Yoneda to a map $\alpha: \mathcal{C}(D,-) \longrightarrow F$, which we show to be an isomorphism. It suffices to verify that $\mathcal{V}_{0}\left(X, \alpha_{C}\right): \mathcal{V}_{0}(X, \mathcal{C}(D, C)) \rightarrow \mathcal{V}_{0}(X, F C)$ is an isomorphism for each $C \in \mathcal{C}$ and each $X \in \mathcal{V}$. The domain here is isomorphic by (3.45) to $\mathcal{C}_{0}(D, X \pitchfork C)$, and the codomain by $(1.25)$ to $V[X, F C]$, which is then isomorphic to $V F(X \pitchfork C)$ since $F$ preserves cotensor products. It is easily verified that, modulo these isomorphisms, $\mathcal{V}_{0}\left(X, \alpha_{C}\right)$ is in fact the isomorphism $\beta_{X \pitchfork C}$.

The case of adjoints follows from this, since $\mathcal{B}_{0}(B, T-)=V(\mathcal{B}(B, T-))_{0}$ by $(1.33)$ and since, if $T$ preserves cotensor products, so does $\mathcal{B}(B, T-)$.

We accordingly take $\mathcal{V}=$ Set for the rest of this section. Recall that, in taking $\mathcal{C}$ to be a Set-category, we are automatically supposing it to be locally small. There are many classical results on the representability of $F: \mathcal{C} \longrightarrow$ Set; and to reproduce them here in detail, with examples of their use, would involve an unjustifiably long digression from our main path of development. Some briefer comments may however be useful. First, whether $\mathcal{C}$ is complete or not, applying Proposition 4.82 to the left Kan extension (4.79) gives:

Proposition $4.86 \mathrm{~F}: \mathcal{C} \longrightarrow$ Set is representable if and only if $d:$ el $F \longrightarrow \mathcal{C}$ has a limit which is preserved by $F$; the representing object is then $\lim d$.

The following proposition is useful in various contexts; it follows at once from the observation in 4.6 that a functor $T: \mathcal{A} \longrightarrow$ el $F$ consists of a functor $S=d T: \mathcal{A} \longrightarrow \mathcal{C}$ and an $\alpha \in \lim F S$ :

Proposition 4.87 If $\mathcal{C}$ admits the conical limits of some class, and $F: \mathcal{C} \longrightarrow$ Set preserves them, then el $F$ also admits such limits, and $d:$ el $F \longrightarrow \mathcal{C}$ preserves and reflects them. In particular, el $F$ is complete if $\mathcal{C}$ is complete and $F$ is continuous.

By 3.4 and Proposition 4.83, $F: \mathcal{C} \longrightarrow$ Set is accessible precisely when it is a small conical colimit of representables. There is a weaker condition - strictly weaker by [29] related to this as finite-generation of an algebra is related to finite-presentability: namely that $F$ be an epimorphic image of a small coproduct of representables; call this weak accessibility. Consider what it means. A map $\mathcal{C}\left(D_{\alpha},-\right) \longrightarrow F$ corresponds by Yoneda to an object $\left(D_{\alpha}, y_{\alpha} \in F D\right)$ of el $F$. Since epimorphisms in $[\mathcal{C}$, Set $]$ are pointwise, to say that $\sum_{\alpha} \mathcal{C}\left(D_{\alpha},-\right) \longrightarrow F$ is epimorphic is to say that every $(C, x) \in \operatorname{el} F$ admits a map from some $\left(D_{\alpha}, y_{\alpha}\right)$. If $\mathcal{S}$ is the small full subcategory of el $F$ determined by the objects 
$\left(D_{\alpha}, y_{\alpha}\right)$, and $J: \mathcal{S} \longrightarrow$ el $F$ is the inclusion, this is to say that the comma-category $J /(C, x)$ is non-empty for each $(C, x)$.

When el $F$ is complete, however - or even finitely complete - so that $(\mathrm{el} F)^{\text {op }}$ is filtered, the non-emptiness of each $J /(C, x)$ implies by Proposition 4.71 the initialness of $J: \mathcal{S} \longrightarrow$ el $F$. Thus from Theorem 4.84, Proposition 4.86, Proposition 4.87, and Theorem 4.67, we get Freyd's General adjoint functor theorem (cf. [28]):

Theorem 4.88 For $F: \mathcal{C} \longrightarrow$ Set where $\mathcal{C}$ is locally-small and complete, the following are equivalent:

(i) F is representable.

(ii) F is continuous and accessible.

(iii) F is continuous and weakly accessible.

Then the representing object for $F$ is $\lim d J$.

The weak accessibility of $F$ is often called the solution-set condition. Since $\mathcal{C}$ is locally small, it can be expressed as follows: there is a small set $\mathcal{T}$ of objects of $\mathcal{C}$ such that, for any $C \in \mathcal{C}$ and any $x \in F C$, there is some $E \in \mathcal{T}$, some $y \in F E$, and some $f: E \longrightarrow C$ with $(F f) y=x$.

Recall that we also have the quite elementary observation from 1.10:

Proposition 4.89 For $F: \mathcal{C} \longrightarrow$ Set the following are equivalent:

(i) F admits a representation $\alpha: \mathcal{C}(D,-) \cong F$ with unit $\eta \in F D$.

(ii) el $F$ has an initial object $(D, \eta)$.

(iii) $\Delta 1:$ el $F \longrightarrow$ Set is representable by $(D, \eta)$.

Applied to $\Delta 1: \mathcal{E} \longrightarrow$ Set for any Set-category $\mathcal{E}$, Theorem 4.88 gives that $\mathcal{E}$ has an initial object if it is complete and has a small full subcategory $\mathcal{S}$ (the "solution set") such that any $E \in \mathcal{E}$ admits a map from some $S \in \mathcal{S}$. Conversely we could have proved Theorem 4.88 alternatively by using Proposition 4.89 and Proposition 4.87 to reduce it to this "initial-object theorem", and by giving a simple direct proof of the latter. In discussing variants of Theorem 4.88, we take this approach for brevity: we describe the corresponding initial-functor theorem, and leave the reader to translate it back to a representability theorem.

An unpublished result of Freyd, given in lectures in 1970, deals with a non-complete $\mathcal{E}$. It asserts that $\mathcal{E}$ has an initial object if and only if (i) $\mathcal{E}$ satisfies the solution-set condition as above, (ii) every $A \in \mathcal{E}$ has a map from some $B \in \mathcal{E}$ all of whose idempotent endomorphisms split, and (iii) every small diagram in $\mathcal{E}$ admits some projective cone over it. The corresponding representability theorem, with $\mathcal{C}$ the (non-complete) stable homotopy category of CW-complexes, gives Brown's result [11] on the representability of cohomology theories. 
Very commonly, $\mathcal{E}$ is not only complete but admits arbitrary (even large) intersections of subobjects; by Proposition 4.87, el $F$ is so if $\mathcal{C}$ is so and if $F$ preserves, besides small limits, all intersections of subobjects. For such an $\mathcal{E}$, the intersection of all the subobjects of $E \in \mathcal{E}$ is an atom - an object with no proper subobject. So the set $\mathcal{S}$ of isomorphismclasses of atoms in $\mathcal{E}$ forms a solution-set if it is small. In many concrete cases, it is easy to find a small cardinal exceeding card $\mathcal{S}$; this is probably the most common way of applying Theorem 4.88 .

The adjoint-functor theorem of Day [15], in its initial-object-theorem form, requires only partial completeness of $\mathcal{E}$ : the existence of equalizers, of pullbacks of monomorphisms, and of arbitrary intersections of subobjects; but it strengthens the solution-set condition to the requirement that there is some $S \in \mathcal{E}$ such that, for each $A \in \mathcal{E}$, there is a monomorphism $A \longrightarrow B$ and a map $S \longrightarrow B$. Then the atom in $S$ is the initial object. Since this refers to no infinite limits except intersections of monomorphisms, it has an analogue [18] in an elementary topos, where such intersections exist in an internal sense.

Day's result also yields a proof of Freyd's Special Adjoint Functor Theorem (cf. [28]):

Theorem 4.90 If the locally-small $\mathcal{C}$ is complete, admits all intersections of subobjects, and has a small cogenerating set, every continuous $F: \mathcal{C} \longrightarrow$ Set is representable.

Proof. It is clear that $\mathcal{E}=\mathrm{el} F$ has a small cogenerating set $\left\{G_{\alpha}\right\}$ in these conditions. The hypothesis of Day's initial-object theorem are clearly satisfied with $S=\prod_{\alpha} G_{\alpha}$ and with $A \longrightarrow B$ the canonical monomorphism $A \longrightarrow \prod_{\alpha}\left(\mathcal{E}\left(G_{\alpha}, A\right) \pitchfork G_{\alpha}\right)$.

Applying this to the existence of a left adjoint to the diagonal $\Delta: \mathcal{C} \longrightarrow[\mathcal{A}, \mathcal{C}]$, we see that $\mathcal{C}$ is cocomplete if it is complete, admits intersections of subobjects, and has a small cogenerating set.

4.10 Existence and characterization of the left Kan extension along a fully-faithful $K: \mathcal{A} \longrightarrow \mathcal{C}$ in terms of cylinders in $\mathcal{C}$ sent to colimits by the $\mathcal{C}(K A,-)$

For cocomplete $\mathcal{B}$, the left Kan extension of $G: \mathcal{A} \longrightarrow \mathcal{B}$ along $K: \mathcal{A} \longrightarrow \mathcal{C}$ exists by (4.13) if $\mathcal{A}$ is small. By Theorem 4.47, it still exists when $\mathcal{A}$ is not small, provided that $K$ is itself a left Kan extension along some $\mathcal{A}^{\prime} \longrightarrow \mathcal{A}$ with $\mathcal{A}^{\prime}$ small. We now turn to existence results when $\mathcal{B}$ is no longer cocomplete, but admits colimits only of some specified class.

Theorem 4.91 The left Kan extension of $G: \mathcal{A} \longrightarrow \mathcal{B}$ along $K: \mathcal{A} \longrightarrow \mathcal{C}$ exists if and only if, for each $C \in \mathcal{C}$, the functor $\widetilde{K} C: \mathcal{A}^{\text {op }} \longrightarrow \mathcal{V}$ can be so expressed as a colimit $F \star Y Q$ of representables that the colimit $F \star G Q$ exists in $\mathcal{B}$; then this latter colimit is $\left(\operatorname{Lan}_{K} G\right) C$.

Proof. Let $\widetilde{K} C$ be so expressible, where $F: \mathcal{L}^{\text {op }} \longrightarrow \mathcal{V}$ and $Q: \mathcal{L} \longrightarrow \mathcal{A}$. Since $(Y Q-) \star$ $G \cong G Q$ by (3.10), we have from (3.23) that $\widetilde{K} C \star G=(F \star Y Q) \star G$ exists, being $F \star((Y Q-) \star G) \cong F \star G Q$. For the converse, we just take the canonical expression (3.17) of $\widetilde{K} C$ as a colimit of representables, corresponding to $F=\widetilde{K} C$ and $Q=1$. 
As an example of this, take $\mathcal{V}=$ Set, let $\left\{G_{x}\right\}_{x \in X}$ be a small set of objects in the cocomplete category $\mathcal{C}$, and let $\mathcal{A}$ be the full subcategory of $\mathcal{C}$ determined by the small coproducts of the $G_{x}$, with $K: \mathcal{A} \longrightarrow \mathcal{C}$ the inclusion. For any $C \in \mathcal{C}$, let $\epsilon: D=$ $\sum_{x \in X} \mathcal{C}\left(G_{x}, C\right) \cdot G_{x} \longrightarrow C$ be the canonical map whose $(x, f)$-component, for $f \in \mathcal{C}\left(G_{x}, C\right)$, is $f$. Write $M_{x}$ for the set of pairs of maps $g, h: G_{x} \longrightarrow D$ such that $\epsilon g=\epsilon h$, and let $\phi, \psi: E=\sum_{x} M_{x} \cdot G_{x} \longrightarrow D$ be the maps whose respective $(x, g, h)$-components are $g$ and $h$. The commutative diagram

$$
E \underset{\psi}{\stackrel{\phi}{\longrightarrow}} D \underset{\epsilon}{\longrightarrow} C
$$

is not in general a coequalizer diagram; but for each $x$ the diagram

$$
\mathcal{C}\left(G_{x}, E\right) \underset{\mathcal{C}(1, \psi)}{\stackrel{\mathcal{C}(1, \phi)}{\longrightarrow}} \mathcal{C}\left(G_{x}, D\right) \underset{\mathcal{C}(1, \epsilon)}{\longrightarrow} \mathcal{C}\left(G_{x}, C\right)
$$

is a split coequalizer diagram. That is to say, there are maps $t: \mathcal{C}\left(G_{x}, C\right) \longrightarrow \mathcal{C}\left(G_{x}, D\right)$ and $s: \mathcal{C}\left(G_{x}, D\right) \longrightarrow \mathcal{C}\left(G_{x}, E\right)$ for which $\mathcal{C}(1, \epsilon) t=1, \mathcal{C}(1, \phi) s=1$, and $\mathcal{C}(1, \psi) s=t \mathcal{C}(1, \epsilon)$; which easily implies that (4.93) is a coequalizer. To wit, $t(f): G_{x} \longrightarrow D$ is the coprojection $i_{x, f}$ of the coproduct, while $s(u): G_{x} \longrightarrow E$ is the coprojection corresponding to the index $\left(x, u, i_{x, \epsilon u}\right)$. Any product of the diagrams (4.93) over a family $\left(x_{j}\right)$ of the indices $x$ is clearly again a split coequalizer; whence it follows that $\widetilde{K}$ sends (4.92) to a coequalizer diagram

$$
\mathcal{A}(-, E) \longrightarrow \mathcal{A}(-, D) \longrightarrow \widetilde{K} C
$$

in $\left[\mathcal{A}^{\text {op }}\right.$, Set $]$. We conclude that the left Kan extension of $G: \mathcal{A} \longrightarrow \mathcal{B}$ along $K: \mathcal{A} \longrightarrow \mathcal{C}$ exists whenever $\mathcal{B}$ admits coequalizers, and is given as the coequalizer of the two maps $G \phi, G \psi: G E \longrightarrow G D$. (We remark that, when (4.92) is a coequalizer diagram for each $C,\left\{G_{x}\right\}$ is said to be a regular generator of $\mathcal{C}$; it is easily verified to be a fortiori a strong generator and a generator.) Clearly Theorem 4.91 could be so modified as to cover the case where $\widetilde{K} C$ was expressed as an iterated colimit of representables. There is a particularly neat and useful result of this kind when $K$ is fully faithful; which we now suppose.

Let $F: \mathcal{L}^{\text {op }} \longrightarrow \mathcal{V}$, let $P: \mathcal{L} \longrightarrow \mathcal{C}$ be a diagram of type $F$, let $C \in \mathcal{C}$, and let $\alpha: F \longrightarrow \mathcal{C}(P-, C)$ be an $(F, C)$-cylinder over $P$, in the language of 3.1. Let us say that $\alpha$ is a $K$-cylinder if the composite cylinder

$$
F \underset{\alpha}{\longrightarrow} \mathcal{C}(P-, C) \underset{\widetilde{K}}{\longrightarrow}\left[\mathcal{A}^{\mathrm{op}}, \mathcal{V}\right](\widetilde{K} P-, \widetilde{K} C)
$$

is a colimit-cylinder, giving

$$
\widetilde{K} C \cong F \star \widetilde{K} P .
$$

For this it certainly suffices that, for each $A \in \mathcal{A}$, the cylinder

$$
F \underset{\alpha}{\longrightarrow} \mathcal{C}(P-, C) \underset{\mathcal{C}(K A,-)}{\longrightarrow}[\mathcal{C}(K A, P-), \mathcal{C}(K A, C)]
$$

be a colimit-cylinder, so that

$$
\mathcal{C}(K A, C) \cong F \star \mathcal{C}(K A, P-)
$$


this is just the pointwise existence of the colimit (4.94); and by 3.3 the pointwiseness is automatic if $\mathcal{L}$ is small.

An expression of $\widetilde{K} C$ as a colimit $F \star Y Q$ of representables, as in Theorem 4.91, has as unit a cylinder $\beta: F \longrightarrow\left[\mathcal{A}^{\mathrm{op}}, \mathcal{V}\right](Y Q-, \widetilde{K} C)$. The codomain of $\beta$ is isomorphic by Yoneda to $(\widetilde{K} C) Q^{\text {op }}=\mathcal{C}(K Q-, C)$; moreover, since $K$ is fully faithful, we have $Y \cong \widetilde{K} K$ by (4.22). It follows that $\beta$ has the form

$$
F \underset{\alpha}{\longrightarrow} \mathcal{C}(K Q-, C) \underset{\widetilde{K}}{\longrightarrow}\left[\mathcal{A}^{\text {op }}, \mathcal{V}\right](\widetilde{K} K Q-, \widetilde{K} C)
$$

in which the second map is in fact an isomorphism. Thus such an expression of $\widetilde{K} C$ as a colimit of representables is the same thing as a $K$-cylinder in which $P$ factorizes through $\mathcal{A}$ as $K Q$.

In this fully-faithful case we have a characterization of $\operatorname{Lan}_{K} G$ as follows:

Theorem 4.97 An extension $S: \mathcal{C} \longrightarrow \mathcal{B}$ of $G: \mathcal{A} \longrightarrow \mathcal{B}$ along the fully-faithful $K: \mathcal{A} \longrightarrow \mathcal{C}$ is $\operatorname{Lan}_{K} G$ (that is, $S$ is canonically isomorphic to $\operatorname{Lan}_{K} S K$ ) if and only if it sends each $K$-cylinder to a colimit-cylinder. If $\mathcal{A}$ is small, it suffices to speak of small $K$-cylinders.

Proof. The canonical expression of $\widetilde{K} C$ as a colimit $\widetilde{K} C \star Y$ of representables corresponds as in (4.96) to a $K$-cylinder $1: \widetilde{K} C \longrightarrow \mathcal{C}(K-, C)$. If $S$ sends this to a colimitcylinder we have $S C \cong \widetilde{K} C \star S K$, which is $\widetilde{K} C \star G$ since $S$ is an extension of $G$; thus $S \cong \operatorname{Lan}_{K} G$. Conversely, if $\operatorname{Lan}_{K} G$ exists, any $K$-cylinder $\alpha$ is, by definition, sent to a colimit-cylinder by $\widetilde{K}$; by (3.23) the functor $-\star G$ preserves the colimit (4.94), since each $\widetilde{K} P L \star G$ exists; hence $\operatorname{Lan}_{K} G=\widetilde{K}-\star G$ sends $\alpha$ to a colimit-cylinder.

Still supposing $K$ fully faithful, consider a family $\Phi=\left(\alpha_{\gamma}: F_{\gamma} \longrightarrow \mathcal{C}\left(P_{\gamma}-, C_{\gamma}\right)\right)_{\gamma \in \Gamma}$ of $K$-cylinders, where $F_{\gamma}: \mathcal{L}_{\gamma}^{\text {op }} \longrightarrow \mathcal{V}$ and $P_{\gamma}: \mathcal{L}_{\gamma} \longrightarrow \mathcal{C}$. Generalizing 3.5, where it was a question of a family of colimits, we say that $\mathcal{C}$ is the closure of $\mathcal{A}$ under the family $\Phi$ if there is no proper replete full subcategory $\mathcal{D}$ of $\mathcal{C}$, containing $\mathcal{A}$, and such that $C_{\gamma} \in \mathcal{D}$ whenever $P_{\gamma}$ takes its values in $\mathcal{D}$.

Theorem 4.98 Let $K: \mathcal{A} \longrightarrow \mathcal{C}$ be fully faithful, and let $\mathcal{C}$ be the closure of $\mathcal{A}$ for a family $\Phi$ of $K$-cylinders as above. Then a functor $S: \mathcal{C} \longrightarrow \mathcal{B}$ is exhibited by $1: S K \longrightarrow S K$ as $\operatorname{Lan}_{K} S K$ if and only if $S$ sends each cylinder of $\Phi$ to a colimit-cylinder. Moreover $\operatorname{Lan}_{K} G$ exists for any $G: \mathcal{A} \longrightarrow \mathcal{B}$ if $\mathcal{B}$ admits $F_{\gamma}$-indexed colimits for each $\gamma \in \Gamma$.

Proof. Theorem 4.97 gives the necessity in the first assertion. For the sufficiency, let $\mathcal{D}$ be the replete full subcategory of $\mathcal{C}$ consisting of those $C$ for which $S: \mathcal{C}(K-, C) \longrightarrow \mathcal{B}(S K-, S C)$ as unit exhibits $S C$ as $\widetilde{K} C \star S K$. Then $\mathcal{A} \subset \mathcal{D}$ since we have canonical isomorphisms $S K A \cong Y A \star S K \cong \widetilde{K} K A \star S K$. If $P_{\gamma}$ takes its values in $\mathcal{D}$, we have a canonical isomorphism $\widetilde{K} P_{\gamma}-\star S K \cong S P_{\gamma}$ by the definition of $\mathcal{D}$; and $\widetilde{K} C_{\gamma} \cong F_{\gamma} \star \widetilde{K} P_{\gamma}$ by (4.94). Hence (3.23) gives $F_{\gamma} \star S P_{\gamma} \cong \widetilde{K} C_{\gamma} \star S K$. Since $F_{\gamma} \star S P_{\gamma} \cong S C_{\gamma}$ by hypothesis, we have $C_{\gamma} \in \mathcal{D}$; thus $\mathcal{D}$ is all of $\mathcal{C}$, as desired. The second assertion is proved by a similar argument, taking $\mathcal{D}$ to consist of those $C$ for which $\widetilde{K} C \star G$ exists. 
For an example of this, let us modify the example following Theorem 4.91. With the notation as there, write $\mathcal{A}^{\prime}$ for the full subcategory of $\mathcal{C}$ determined by the finite coproducts of the $G_{x}$. Since every coproduct is the filtered colimit of the finite coproducts of its summands, every object of $\mathcal{A}$ is a filtered colimit of objects of $\mathcal{A}^{\prime}$. Suppose now that each $G_{x}$ is finitely presentable - meaning that each $\mathcal{C}\left(G_{x},-\right): \mathcal{C} \longrightarrow$ Set preserves filtered colimits. Then, since filtered colimits commute with finite limits in Set by Theorem 4.73, each object of $\mathcal{A}^{\prime}$ is finitely presentable; and thus the filtered colimits in question are preserved by $\widetilde{Z}: \mathcal{C} \longrightarrow\left[\mathcal{A}^{\text {op }}\right.$, Set $]$, where $Z$ is the inclusion $\mathcal{A}^{\prime} \longrightarrow \mathcal{A} \longrightarrow \mathcal{C}$; that is, they correspond to $Z$-cylinders. It now follows from Theorem 4.98 that any $G: \mathcal{A}^{\prime} \longrightarrow \mathcal{B}$ admits a left Kan extension along $Z: \mathcal{A}^{\prime} \longrightarrow \mathcal{C}$ if $\mathcal{B}$ admits coequalizers and filtered colimits.

We end with a result, a special case of which we have already used in Theorem 4.51:

Theorem 4.99 Let $K: \mathcal{A} \longrightarrow \mathcal{C}$ be fully faithful, and denote by $[\mathcal{A}, \mathcal{B}]^{\prime}$ the full subcategory of $[\mathcal{A}, \mathcal{B}]$ determined by those $G$ for which $\operatorname{Lan}_{K} G$ exists. Then $\operatorname{Lan}_{K}:[\mathcal{A}, \mathcal{B}]^{\prime} \longrightarrow[\mathcal{C}, \mathcal{B}]$ is fully faithful; so that we have an equivalence $[\mathcal{A}, \mathcal{B}]^{\prime} \simeq[\mathcal{C}, \mathcal{B}]^{\ell}$, where the latter is the full subcategory of $[\mathcal{C}, \mathcal{B}]$ determined by the functors isomorphic to some $\operatorname{Lan}_{K} G$; the inverse to this equivalence is composition with $K$. If $\mathcal{A}$ is small, it follows that $[\mathcal{C}, \mathcal{B}]^{\ell}$ is a $\mathcal{V}$-category.

Proof. We have by Theorem 4.38 that $[\mathcal{C}, \mathcal{B}]\left(\operatorname{Lan}_{K} G, \operatorname{Lan}_{K} G^{\prime}\right) \cong[\mathcal{A}, \mathcal{B}]\left(G,\left(\operatorname{Lan}_{K} G^{\prime}\right) K\right)$, which by Proposition 4.23 is isomorphic to $[\mathcal{A}, \mathcal{B}]\left(G, G^{\prime}\right)$.

Of course this fully-faithfulness is just a special case of the observation in 1.11 following (1.53), to which we could have appealed: for we are dealing with an adjunction $\operatorname{Lan}_{K} \dashv$ $[K, 1]$ whose unit $\phi$ is an isomorphism. In practice we use the equivalence $[\mathcal{A}, \mathcal{B}]^{\prime} \simeq[\mathcal{C}, \mathcal{B}]^{\ell}$ chiefly when $[\mathcal{A}, \mathcal{B}]^{\prime}$ is all of $[\mathcal{A}, \mathcal{B}]$, as for instance under the hypotheses of Theorem 4.98 or when $\mathcal{A}$ is small and $\mathcal{B}$ is cocomplete. 


\section{Chapter 5}

\section{Density}

\subsection{Definition of density, and equivalent formulations}

A function $k: A \longrightarrow C$, not necessarily continuous, between hausdorff spaces is dense (in the sense that its image is a dense subset) precisely when any continuous map from $C$ into another hausdorff space $B$ is uniquely determined by its composite with $k$; that is, when $\operatorname{Set}(k, 1): \operatorname{Haus}(C, B) \longrightarrow \operatorname{Set}(A, B)$ is injective. We give an analogous definition of density for a $\mathcal{V}$-functor $K: \mathcal{A} \longrightarrow \mathcal{C}$.

For a $\mathcal{V}$-category $\mathcal{C}$, the functor category $[\mathcal{C}, \mathcal{B}]$ exists at least as a $\mathcal{V}^{\prime}$-category for some suitable extension $\mathcal{V}^{\prime}$ of $\mathcal{V}$ as in 3.12. Consider those $S: \mathcal{C} \longrightarrow \mathcal{B}$ which preserve every colimit existing in $\mathcal{C}$ whose indexing-type $F: \mathcal{A}^{\mathrm{op}} \longrightarrow \mathcal{V}$ has domain $\mathcal{A}^{\text {op }}$; these constitute a full subcategory $\mathcal{A}$-Cocts $[\mathcal{C}, \mathcal{B}]$, which if $\mathcal{A}$ is small contains the full subcategory $\operatorname{Cocts}[\mathcal{C}, \mathcal{B}]$ of those $S$ preserving all small colimits that exist.

Theorem 5.1 For a $\mathcal{V}$-functor $K: \mathcal{A} \longrightarrow \mathcal{C}$ the following are equivalent:

(i) For any $\mathcal{B}$, the restriction to $\mathcal{A}$-Cocts $[\mathcal{C}, \mathcal{B}]$ of the functor $[K, 1]:[\mathcal{C}, \mathcal{B}] \longrightarrow[\mathcal{A}, \mathcal{B}]$ is fully faithful.

(ii) The functor $\widetilde{K}: \mathcal{C} \rightarrow\left[\mathcal{A}^{\mathrm{op}}, \mathcal{V}\right]$ is fully faithful.

(iii) For each $C, D \in \mathcal{C}$ the map $\widetilde{K}: \mathcal{C}(C, D) \longrightarrow\left[\mathcal{A}^{\text {op }}, \mathcal{V}\right](\mathcal{C}(K-, C), \mathcal{C}(K-, D))$ is an isomorphism.

(iv) For each $C \in \mathcal{C}$, the identity cylinder $1: \mathcal{C}(K-, C) \longrightarrow \mathcal{C}(K-, C)$ as unit exhibits $C$ as $\widetilde{K} C \star K$.

(v) The identity $1: K \longrightarrow 1_{\mathcal{C}} K$ exhibits $1_{\mathcal{C}}$ as $\operatorname{Lan}_{K} K$.

(vi) Some isomorphism $\phi: K \longrightarrow 1_{\mathcal{C}} K$ exhibits $1_{\mathcal{C}}$ as $\operatorname{Lan}_{K} K$.

Proof. Because a representable $\mathcal{C}^{\text {op }} \longrightarrow \mathcal{V}$ preserves all limits that exist, its dual $\mathcal{C} \longrightarrow \mathcal{V}^{\text {op }}$ preserves all colimits that exist in $\mathcal{C}$. Since the Yoneda embedding is fully faithful, and since $\widetilde{K}$ is the composite

$$
\mathcal{C} \underset{Y}{\longrightarrow}\left[\mathcal{C}^{\mathrm{op}}, \mathcal{V}\right]_{\left[K^{\mathrm{op}}, 1\right]}^{\longrightarrow}\left[\mathcal{A}^{\mathrm{op}}, \mathcal{V}\right]
$$


it follows that (i) implies (ii). The direct translation (iii) of (ii) is equivalent by (3.5) to (iv), which is in turn equivalent by (4.18) to (v), itself a special case of (vi); so that it remains only to show that (vi) implies (i). Suppose then that $S: \mathcal{C} \longrightarrow \mathcal{B}$ is $\mathcal{A}$-cocontinuous; it thus preserves the colimit $\widetilde{K} C \star K$, and hence the left Kan extension in (vi), so that $S \phi: S K \longrightarrow S K$ exhibits $S$ as $\operatorname{Lan}_{K}(S K)$. By Theorem 4.38 we therefore have for any $S^{\prime}: \mathcal{C} \longrightarrow \mathcal{B}$ a representation $[\mathcal{C}, \mathcal{B}]\left(S, S^{\prime}\right) \cong[\mathcal{A}, \mathcal{B}]\left(S K, S^{\prime} K\right)$, whose unit is $S \phi$. By (1.48), this isomorphism is the composite of $[K, 1]:[\mathcal{C}, \mathcal{B}]\left(S, S^{\prime}\right) \longrightarrow[\mathcal{A}, \mathcal{B}]\left(S K, S^{\prime} K\right)$ and $[\mathcal{A}, \mathcal{B}](S \phi, 1):[\mathcal{A}, \mathcal{B}]\left(S K, S^{\prime} K\right) \longrightarrow[\mathcal{A}, \mathcal{B}]\left(S K, S^{\prime} K\right)$; the latter being an isomorphism since $\phi$ is, so is the former - which proves (i).

A $\mathcal{V}$-functor $K: \mathcal{A} \longrightarrow \mathcal{C}$ satisfying these equivalent conditions is said to be dense. (The original term used by Isbell, who introduced the concept for $\mathcal{V}=$ Set in [35], was left adequate.) For the dual notion (Isbell's right adequate) we use codense; $K: \mathcal{A} \longrightarrow \mathcal{C}$ is codense if $K^{\mathrm{op}}: \mathcal{A}^{\mathrm{op}} \longrightarrow \mathcal{C}^{\mathrm{op}}$ is dense. In the important special case where the dense $K: \mathcal{A} \longrightarrow \mathcal{C}$ is the inclusion of a full subcategory, we merely say that $\mathcal{A}$ is dense in $\mathcal{C}$, leaving $K$ to be understood; and if $\mathcal{A}$ is the one-object full subcategory $\{A\}$, we say that $A$ is dense in $\mathcal{C}$.

It is clear that the density of $K$ depends only on "the equivalence class of $K$ in the 2-category of $\mathcal{V}$-functors"; that is, if $K$ is dense, so is the composite

$$
\overline{\mathcal{A}} \simeq \mathcal{A} \underset{K^{\prime}}{\longrightarrow} \mathcal{C} \simeq \overline{\mathcal{C}}
$$

where the outer functors are equivalences and where $K^{\prime}$ is isomorphic to $K$.

When $C$ admits tensor products, the criterion (v) for the density of $K: \mathcal{A} \longrightarrow \mathcal{C}$ becomes by (4.25) the requirement that the canonical map $\mathcal{C}(K A, C) \otimes K A \rightarrow C$ express $C$ as the coend

$$
C \cong \int^{A} \mathcal{C}(K A, C) \otimes K A
$$

while when $\mathcal{V}=$ Set it becomes by (4.27) the requirement that the canonical cone express $C$ as the colimit

$$
C \cong \operatorname{colim}(K / C \underset{d}{\longrightarrow} \mathcal{A} \underset{K}{\longrightarrow} \mathcal{C}) .
$$

If $K: \mathcal{A} \longrightarrow \mathcal{C}$ is dense, applying $V: \mathcal{V}_{0} \longrightarrow$ Set to the isomorphism in Theorem 5.1(iii) above gives a bijection of sets

$$
\mathcal{C}_{0}(C, D) \cong\left[A^{\mathrm{op}}, \mathcal{V}\right]_{0}(\mathcal{C}(K-, C), \mathcal{C}(K-, D)),
$$

whose meaning is that every $\mathcal{V}$-natural transformation $\alpha: \mathcal{C}(K-, C) \longrightarrow \mathcal{C}(K-, D)$ is of the form $\mathcal{C}(K-, f)$ for a unique $f: C \longrightarrow D$. It is clear that (5.5) is in turn equivalent to the density of $K$ if $V$ is conservative, and in particular in the classical case $\mathcal{V}=$ Set. It is also equivalent to density if $\mathcal{C}$ is cotensored; or even if $\mathcal{C}$ admits cotensor products $X_{\beta} \pitchfork C$ for some set $\left\{X_{\beta}\right\}$ constituting a strong generator of $\mathcal{V}_{0}$ : for if we write (5.5) with $X \pitchfork D$ in place of $D$, we easily get $\mathcal{V}_{0}(X, \mathcal{C}(C, D)) \cong \mathcal{V}_{0}\left(X,\left[\mathcal{A}^{\text {op }}, \mathcal{V}\right](\mathcal{C}(K-, C), \mathcal{C}(K-, D))\right)$. Yet in general (5.5) is strictly weaker than density: take $\mathcal{V}=\mathbf{C a t}$, let $\mathcal{C}$ be the 2-category (3.54), and let $K$ be the inclusion of the full subcategory $\{1\}$; we have the bijection (5.5) but not the isomorphism in $\mathcal{V}$ of Theorem 5.1 (iii). 
After first surveying various examples and elementary properties of density, we shall return in 5.11 below to the study of the important map $[K, 1]: \operatorname{Cocts}[\mathcal{C}, \mathcal{B}] \longrightarrow[\mathcal{A}, \mathcal{B}]$ of Theorem 5.1(i).

\subsection{Composability and cancellability properties of dense functors}

We begin by considering the composability and cancellation properties of dense functors. The positive results below are for any $\mathcal{V}$, while the counter-examples are all for $\mathcal{V}=$ Set; the assertions about the density or non-density of particular functors in the counterexamples are easily verified using (5.5). We consider a diagram

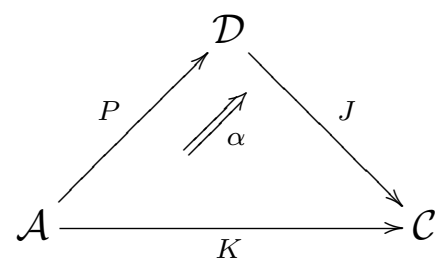

in which $\alpha$ is an isomorphism, so that $K \cong J P$. First, the density of $P$ and of $J$, even if both are fully faithful, does not imply the density of $J P$. For an example, let $\mathcal{A}=1$, let $\mathcal{D}$ be the category of finite sets, and let $\mathcal{C}$ be the functor category $\left[\mathcal{D}^{\text {op }}\right.$, Set $]$; let $P: 1 \longrightarrow \mathcal{D}$ correspond to the object $1 \in \mathcal{D}$, and let $J: \mathcal{D} \longrightarrow\left[\mathcal{D}^{\text {op }}\right.$, Set $]$ be the Yoneda embedding.

Again, the density of $P$ and of $J$ does not imply the density of $J P$ when $P$ is essentially surjective and $J$ is fully faithful; that is, when (5.6) is the factorization of $K$ through its full image $\mathcal{D}$ in $\mathcal{C}$. For an example, let $\mathcal{A}$ be the discrete category identified with the set 2, let $\mathcal{D}$ be the unit category 1 , let $\mathcal{C}=$ Set, and let $J$ be the functor $1 \rightarrow$ Set corresponding to the object $1 \in$ Set.

The following observation leads to some positive results:

Proposition 5.7 If the isomorphism $\alpha$ in (5.6) exhibits $J$ as $\operatorname{Lan}_{P} K$, then $J$ is dense if and only if $K$ is dense.

Proof. Applying Theorem 4.7 to the diagram

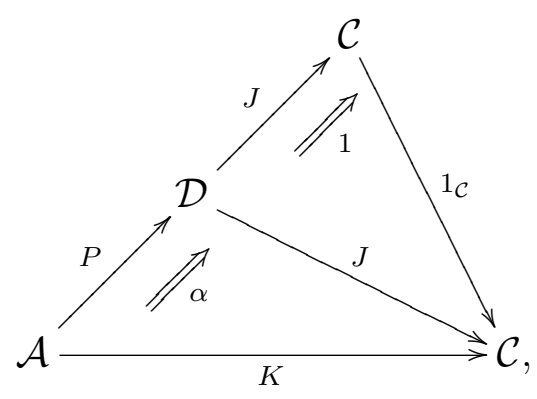

we conclude that 1 exhibits $1_{\mathcal{C}}$ as $\operatorname{Lan}_{J} J$ if and only if $\alpha$ exhibits $1_{\mathcal{C}}$ as $\operatorname{Lan}_{J P} K$. Pasting to the top edge of (5.8) the isomorphism $\alpha^{-1}: J P \longrightarrow K$, we see that $\alpha$ exhibits $1_{\mathcal{C}}$ as $\operatorname{Lan}_{J P} K$ if and only if 1 exhibits $1_{\mathcal{C}}$ as $\operatorname{Lan}_{K} K$. The conclusion now follows from Theorem 5.1(v). 
To say that $\alpha$ exhibits $J$ as $\operatorname{Lan}_{P} K$ is clearly, since $\alpha$ is an isomorphism, to say that 1 exhibits $J$ as $\operatorname{Lan}_{P} J P$. Now if $P$ is dense, so that 1 exhibits $1_{\mathcal{D}}$ as $\operatorname{Lan}_{P} P$, this is by 4.1 to say that $J$ preserves the Kan extension $\operatorname{Lan}_{P} P$. Thus Proposition 5.7 gives:

Proposition 5.9 If $P$ is dense and if $J$ preserves $\operatorname{Lan}_{P} P$, then $J$ is dense if and only if $K \cong J P$ is dense.

If $P$ is fully faithful and if, for any $K: \mathcal{A} \longrightarrow \mathcal{C}$, we define $J: \mathcal{D} \longrightarrow \mathcal{C}$ as $\operatorname{Lan}_{P} K$, supposing this to exist, the unit $\alpha$ of this Kan extension is necessarily an isomorphism by Proposition 4.23. So Proposition 5.7 gives:

Proposition 5.10 If $P: \mathcal{A} \longrightarrow \mathcal{D}$ is fully faithful and if $J: \mathcal{D} \longrightarrow \mathcal{C}$ is $\operatorname{Lan}_{P} K$ for some $K: \mathcal{A} \longrightarrow \mathcal{C}$, then $J$ is dense if and only if $K$ is dense.

A simple cancellability result is given by:

Proposition 5.11 If $P$ is essentially surjective and $K \cong J P$ is dense, then $J$ is dense. In particular, if $K: \mathcal{A} \longrightarrow \mathcal{C}$ is dense, the full image of $K$ is a dense full subcategory of $\mathcal{C}$.

ProOF. $\widetilde{K}$ being fully faithful, so is $\widetilde{J P}$, which is clearly the composite

$$
\mathcal{C} \underset{\widetilde{J}}{\longrightarrow}\left[\mathcal{D}^{\mathrm{op}}, \mathcal{V}\right] \underset{\left[P^{\mathrm{op}, 1]}\right.}{\longrightarrow}\left[\mathcal{A}^{\mathrm{op}}, \mathcal{V}\right]
$$

Thus, for $C, D \in \mathcal{C}$, the composite $\left[P^{\text {op }}, 1\right]_{\widetilde{J} C, \widetilde{J} D} \cdot \widetilde{J}_{C D}$ is an isomorphism. The desired result that $\widetilde{J}_{C D}$ is an isomorphism will follow if $\left[P^{\mathrm{op}}, 1\right]_{T S}$ is shown to be a monomorphism in $\mathcal{V}_{0}$ (or in $\mathcal{V}_{0}^{\prime}$ if $\mathcal{D}$ is large) for all $T, S: \mathcal{D}^{\text {op }} \longrightarrow \mathcal{V}$.

We have by (2.26) the commutative diagram

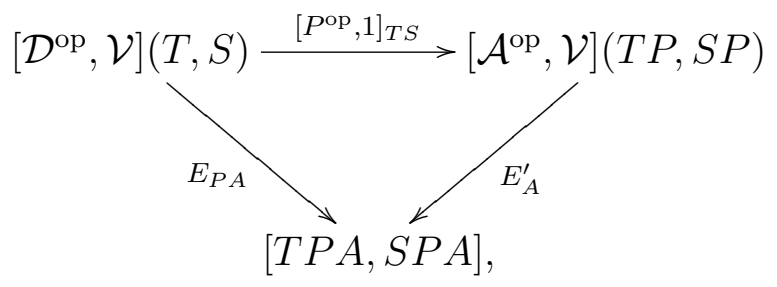

where $E$ and $E^{\prime}$ are the respective evaluations. By the definition in 2.1 of the end in $V$, the family of maps $\left(E_{D}:\left[\mathcal{D}^{\text {op }}, \mathcal{V}\right](T, S) \longrightarrow[T D, S D]\right)_{D \in \mathcal{D}}$ is jointly monomorphic. Since $\mathrm{P}$ is essentially surjective, the same is true of the family $\left(E_{P A}\right)_{A \in \mathcal{A}}$. It now follows from (5.12) that $\left[P^{\mathrm{op}}, 1\right]_{T S}$ is monomorphic, as desired.

Note that, in contrast, the essential surjectivity of $P$, combined with the density of $K=J P$, does not imply the density of $P$. For an example, let $\mathcal{D}$ be any category that is not a preordered set, let $\mathcal{A}$ be the coproduct $\mathcal{D}+\mathcal{D}$, let $P: \mathcal{A} \longrightarrow \mathcal{D}$ be the codiagonal functor, and let $\mathcal{C}=1$. As for the second assertion of the proposition above, we have seen in the example preceding Proposition 5.7 that the converse is false; $K: \mathcal{A} \longrightarrow \mathcal{C}$ need not be dense when its full image $\mathcal{D}$ is dense in $\mathcal{C}$, even if $P: \mathcal{A} \longrightarrow \mathcal{D}$ is also dense.

There is however a strong cancellability result when $J$ is fully faithful:

Theorem 5.13 Let $J$ be fully faithful and let $K \cong J P$ be dense. Then both $P$ and $J$ are dense. Moreover the isomorphism $K \cong J P$ exhibits $J$ as $\operatorname{Lan}_{P} K$. 
Proof. Using (5.2), suppose for simplicity that $K=J P$. Because $J$ is fully faithful, we have $\widetilde{P} \cong \widetilde{K} J$ by (4.49). Since $\widetilde{K}$ and $J$ are each fully faithful by hypothesis, so is $\widetilde{P}$; so that $P$ is dense by Theorem 5.1 (ii).

Again, because $\widetilde{K}$ and $J$ are both fully faithful, we have the composite $\mathcal{V}$-natural isomorphism

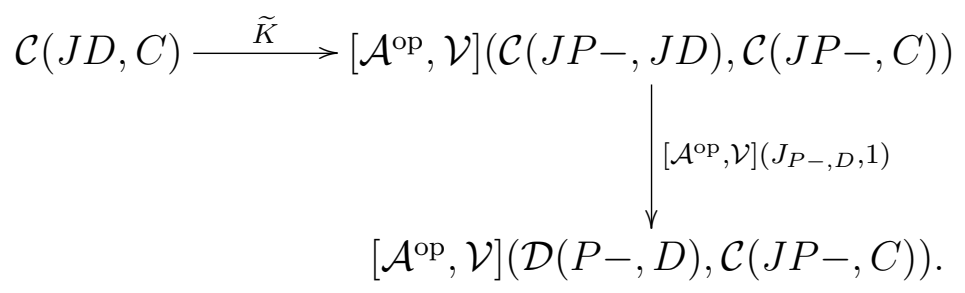

By (3.5), this isomorphism exhibits JD as $\widetilde{P} D \star J P$, with unit

$$
J: \mathcal{D}(P-, D) \longrightarrow \mathcal{C}(J P-, J D) .
$$

By the dual of Theorem 4.6(ii), this is to say that $1: K \longrightarrow J P$ exhibits $J$ as $\operatorname{Lan}_{P} K$. That $J$ is dense now follows from Proposition 5.7.

There are no similar cancellability results when $K=J P$ is fully faithful and dense, and $P$ too is fully faithful. Here, even if $P$ is dense, $J$ need not be: for an example, let $\mathcal{A}=1$, let $\mathcal{D}$ be the ordered set $\mathbf{2}=(0 \longrightarrow 1)$, let $P: 1 \longrightarrow \mathbf{2}$ have image 1 , let $\mathcal{C}=$ Set, and let $J: \mathbf{2} \longrightarrow$ Set send $(0 \longrightarrow 1)$ to the unique map $(\mathbf{2} \longrightarrow 1)$. Furthermore, even if $J$ is dense, $P$ need not be. Let $\mathcal{A}=1$, let $\mathcal{D}$ be the category of the finite ordinals $\mathbf{0}, \mathbf{1}, \mathbf{2}, \mathbf{3}, \ldots$ and increasing functions, let $P: 1 \longrightarrow \mathcal{D}$ have image 1 , let $\mathcal{C}=$ Set, and let $J: \mathcal{D} \longrightarrow$ Set be the (non-full) inclusion.

\subsection{Examples of density; comparison with strong generation}

Since a fully-faithful $\mathcal{V}$-functor is a fortiori conservative, comparison of Theorem 5.1 with 3.6 shows that a dense $K: \mathcal{A} \longrightarrow \mathcal{C}$ is strongly generating; or rather, since we defined "strongly generating" only when $\mathcal{A}$ was small, that this is the case for small $\mathcal{A}$. While we saw in 3.6, however, that $K: \mathcal{A} \longrightarrow \mathcal{C}$ is strongly generating precisely when its full image in $\mathcal{C}$ is so, we have seen in Proposition 5.11 and in the remark preceding Theorem 5.13 that the density of $K$ is strictly stronger than the density of its full image. At any rate, it is clear that $\mathcal{C}$ admits some dense $K: \mathcal{A} \longrightarrow \mathcal{C}$ with $\mathcal{A}$ small if and only if it admits a small dense full subcategory; which latter is then a fortiori a strong generator. (We shall abbreviate "small dense full subcategory" to "small dense subcategory" in appropriate contexts.)

When $\mathcal{V}=$ Set, if $K: \mathcal{A} \longrightarrow \mathcal{C}$ is dense with $\mathcal{C}$ cocomplete and $\mathcal{A}$ small, (5.3) and the dual of (3.68) show the canonical map $\sum_{A} \mathcal{C}(K A, C) \cdot K A \rightarrow C$ to be a coequalizer; whence it easily follows that the objects $\{K A\}_{A \in \mathcal{A}}$ constitute a regular generator for $\mathcal{C}$ in the sense of 4.10. However density is much stronger than being a strong generator, or even a regular one; and this even for $\mathcal{V}=$ Set.

In the category Comp of compact hausdorff spaces, for instance, the single space 1 is a regular generator - for it is clearly a generator, and every epimorphism in Comp 
is a coequalizer. Yet no small subcategory $\mathcal{A}$ of Comp is dense. For let $\theta$ be a regular cardinal exceeding card $A$ for each $A \in \mathcal{A}$, and let $\Omega$ be the set of ordinals $\leq \theta$, made into a compact hausdorff space by giving it the order topology. Let $2=\{0,1\}$ be the discrete two-element space, and define $f: \Omega \longrightarrow 2$ by $f(\theta)=1, f(\alpha)=0$ for $\alpha<\theta$. Although $f$ is not continuous, composition with $f$ defines a map $\phi_{A}: \operatorname{Comp}(A, \Omega) \longrightarrow \operatorname{Comp}(A, 2)$ for each $A \in \mathcal{A}$; because for any continuous $h: A \longrightarrow \Omega$, the subset $h^{-1}(\theta)$ is closed since $\{\theta\}$ is closed, while $h^{-1}(\Omega-\{\theta\})$ is closed by the choice of $\theta$. Because $\phi_{A}$ is clearly natural in $A \in \mathcal{A}$, it would if $\mathcal{A}$ were dense be induced by some continuous $g: \Omega \longrightarrow 2$. Consideration of constant maps $A \longrightarrow \Omega$ gives the contradiction $g=f$ if some $A$ is non-empty; while the empty set 0 is clearly not dense.

By Theorem 5.1(ii), each dense $K: \mathcal{A} \longrightarrow \mathcal{C}$ exhibits $\mathcal{C}$, to within equivalence, as a full subcategory of $\left[\mathcal{A}^{\mathrm{op}}, \mathcal{V}\right]$. We can characterize the subcategories $\mathcal{C}$ of $\left[\mathcal{A}^{\mathrm{op}}, \mathcal{V}\right]$ that arise in this way, and recapture $K$ from $\mathcal{C}$ :

Proposition 5.14 The inclusion $J: \mathcal{C} \longrightarrow\left[\mathcal{A}^{\mathrm{op}}, \mathcal{V}\right]$ of a full subcategory is isomorphic to $\widetilde{K}$ for some (necessarily dense) $K: \mathcal{A} \longrightarrow \mathcal{C}$ if and only if each representable $\mathcal{A}(-, A) \in$ $\left[\mathcal{A}^{\mathrm{op}}, \mathcal{V}\right]$ admits a reflexion in $\mathcal{C}$. Then $K A$ is the reflexion of $\mathcal{A}(-, A)$, and $K$ is unique to within isomorphism.

Proof. We are asking for a $\mathcal{V}$-natural isomorphism $J C \cong \mathcal{C}(K-, C)$, or $(J C) A \cong$ $\mathcal{C}(K A, C)$. Since $(J C) A \cong\left[\mathcal{A}^{\text {op }}, \mathcal{V}\right](\mathcal{A}(-, A), J C)$ by Yoneda, this is precisely to ask that each $\mathcal{A}(-, A)$ admit a reflexion $K A$ in $\mathcal{C}$; whereupon $K$ has by 1.10 a unique $\mathcal{V}$-functor structure.

This has a simple consequence for cocomplete $\mathcal{C}$ and small $\mathcal{A}$ :

Proposition 5.15 Let $\mathcal{A}$ be small. Then $\mathcal{C}$ is equivalent to a reflective full subcategory of $\left[\mathcal{A}^{\mathrm{op}}, \mathcal{V}\right]$ if and only if $\mathcal{C}$ is cocomplete and there is a dense functor $K: \mathcal{A} \longrightarrow \mathcal{C}$. To within isomorphism, the "inclusion" $J: \mathcal{C} \longrightarrow\left[\mathcal{A}^{\mathrm{op}}, \mathcal{V}\right]$ is $\widetilde{K}$, while $K$ is the composite of the Yoneda embedding $Y: \mathcal{A} \longrightarrow\left[\mathcal{A}^{\mathrm{op}}, \mathcal{V}\right]$ and the left adjoint $S:\left[\mathcal{A}^{\mathrm{op}}, \mathcal{V}\right] \longrightarrow \mathcal{C}$ of $J$. Such $a \mathcal{C}$ is complete.

Proof. If $\mathcal{C}$ is reflective in $\left[\mathcal{A}^{\mathrm{op}}, \mathcal{V}\right]$, with reflexion $S \dashv J$, then certainly each $\mathcal{A}(-, A)$ admits a reflexion $K A=S Y A$ in $\mathcal{C}$; whence by Proposition 5.14 we have $J \cong \widetilde{K}$ for the dense $K=S Y: \mathcal{A} \longrightarrow \mathcal{C}$. Moreover $\mathcal{C}$ is cocomplete by $3.5,\left[\mathcal{A}^{\mathrm{op}}, \mathcal{V}\right]$ being cocomplete by 3.3 and 3.74. Conversely, if $\mathcal{C}$ is cocomplete and $K: \mathcal{A} \longrightarrow \mathcal{C}$ dense, the fully-faithful $\widetilde{K}: \mathcal{C} \longrightarrow\left[\mathcal{A}^{\text {op }}, \mathcal{V}\right]$ has by Theorem 4.51 the left adjoint $-\star K$; and $\mathcal{C}$ is complete by 3.5 .

Another simple case is that of a fully-faithful $K$ :

Proposition $5.16 \mathcal{C}$ is equivalent to a full subcategory of $\left[\mathcal{A}^{\mathrm{op}}, \mathcal{V}\right]$ containing the representables if and only if there is a dense fully-faithful $K: \mathcal{A} \longrightarrow \mathcal{C}$. Then, to within isomorphism, the "inclusion" $J: \mathcal{C} \longrightarrow\left[\mathcal{A}^{\mathrm{op}}, \mathcal{V}\right]$ is $\widetilde{K}$, while the inclusion of $\mathcal{A}$ (identified with the representables) into $\mathcal{C}$ is $K$.

Proof. If $\mathcal{C}$ contains the representables, each $\mathcal{A}(-, A)$ is its own reflexion in $\mathcal{C}$; whence by Proposition 5.14, the inclusion $J: \mathcal{C} \longrightarrow\left[\mathcal{A}^{\text {op }}, \mathcal{V}\right]$ is isomorphic to $\widetilde{K}$ where $K: \mathcal{A} \longrightarrow \mathcal{C}$ is the inclusion. Conversely, if $K: \mathcal{A} \longrightarrow \mathcal{C}$ is fully faithful and dense, (4.22) gives $\widetilde{K} K \cong$ 
$Y: \mathcal{A} \longrightarrow\left[\mathcal{A}^{\mathrm{op}}, \mathcal{V}\right]$, so that $\mathcal{C}$ is equivalent to a full subcategory of $\left[\mathcal{A}^{\mathrm{op}}, \mathcal{V}\right]$ containing the representables.

As extreme cases of Proposition 5.16, we have the density of $1: \mathcal{A} \rightarrow \mathcal{A}(\widetilde{1}$ here being the fully-faithful $\left.Y: \mathcal{A} \longrightarrow\left[\mathcal{A}^{\text {op }}, \mathcal{V}\right]\right)$, and the density of $Y: \mathcal{A} \longrightarrow\left[\mathcal{A}^{\mathrm{op}}, \mathcal{V}\right](\widetilde{Y}$ here by $(4.30)$ being $\left.1:\left[\mathcal{A}^{\mathrm{op}}, \mathcal{V}\right] \longrightarrow\left[\mathcal{A}^{\mathrm{op}}, \mathcal{V}\right]\right)$. If in this latter we take $\mathcal{A}=\mathcal{I}$, which we may identify with the full subcategory $\{I\}$ of $\mathcal{V}$, we observe that:

The single object I is always dense in $\mathcal{V}$.

For instance, 1 is dense in Set, the infinite cyclic group $\mathbf{Z}$ is dense in $\mathbf{A b}$, and $\mathbf{1}$ is dense in Cat.

The assertion (5.17) and these examples of it must be interpreted strictly in accordance with our conventions; there is no suggestion that the single object $I$ is necessarily dense in the ordinary category $\mathcal{V}_{0}$, when $\mathcal{V} \neq$ Set: cf. 3.9 above. For instance, although $\mathbf{Z}$ is dense in the $\mathbf{A b}$-category $\mathbf{A b}$, it is not dense in the ordinary category $\mathbf{A} \mathbf{b}_{0}$, although it is a regular generator there. In fact, the single object $\mathbf{Z} \oplus \mathbf{Z}$ is dense in $\mathbf{A b}_{0}$; this is a special case of the fact (cf. Isbell [35]) that, if $\mathcal{C}$ is an equational category of algebras whose basic operations all have arity $\leq n$, the free algebra $F n$ on $n$ generators is dense in $\mathcal{C}$. (Once again, these assertions of density and of non-density for particular functors in the case $\mathcal{V}=$ Set are easily verified using (5.5).)

Again, although 1 is dense in the Cat-category Cat, it is not even a generator in the ordinary category $\mathbf{C a t}_{0}$. The ordered-set category $\mathbf{2}$ is clearly a strong generator in $\mathbf{C a t}_{0}$; but it is not a regular generator, since $\operatorname{Cat}_{0}(\mathbf{2}, A) \cdot \mathbf{2} \longrightarrow A$ is not a coequalizer if $A$ has one object and has a single non-identity map $e$ with $e^{2}=e$. Hence a fortiori $\mathbf{2}$ is not dense in $\mathbf{C a t}_{0}$. It is easily verified, however, that the ordered set $\mathbf{3}=\{0,1,2\}$ is dense in $\mathrm{Cat}_{0}$.

It is in fact possible, in contrast to (5.17), that $\mathcal{V}_{0}$ admit no small dense subcategory $\mathcal{A}$ whatsoever. An example is the cartesian closed category $\mathcal{V}=$ CGTop of compactlygenerated topological spaces. If $\mathcal{A}$ is a small full subcategory (which we may assume to contain some nonempty space), let $\theta$ be a regular cardinal exceeding card $A$ for each $A \in \mathcal{A}$, and form the compact hausdorff space $\Omega$ as in the example preceding Proposition 5.14. By the argument used there, the non-closed set $\Omega-\{\theta\}$ has a closed inverse image under each continuous $h: A \longrightarrow \Omega$ with $A \in \mathcal{A}$; hence the canonical surjective map $\sum_{A \in \mathcal{A}} \mathcal{A}(A, \Omega) \cdot A \longrightarrow \Omega$ is not a topological quotient map. It factorizes therefore through the set $\Omega$ with the strictly-finer quotient topology (easily seen to be compactly generated), and thus through a proper subobject of $A$. Hence $\mathcal{A}$ is not even a strong generator, much less dense.

We observed in 3.6 that a $\mathcal{V}$-functor $K: \mathcal{A} \longrightarrow \mathcal{C}$ is certainly strongly generating if the ordinary functor $K_{0}: \mathcal{A}_{0} \longrightarrow \mathcal{C}_{0}$ is so. The analogue for density is false; if $\mathcal{V}$ is Cat and $\mathcal{C}$ is the 2-category of (3.54), the object 1 is dense in $\mathcal{C}_{0}$ but not in $\mathcal{C}$. Even more is true: a $\mathcal{V}$-category $\mathcal{C}$ may admit no small dense subcategory at all, although $\mathcal{C}_{0}$ admits one. For an example of this, take $\mathcal{V}=$ Cat again, and let $\mathcal{C}_{0}=$ Set, which has the small dense subcategory 1 . Let the category $\mathcal{C}(X, Y)$ be discrete unless $X$ is the empty set and $Y$ is not. 
The category $\mathcal{C}(0, Y)$ has only one object, $t_{Y}$ say, and is therefore to be a monoid: let it be the free monoid on the symbols $\alpha_{Y}$, where $\alpha$ runs through all cardinals less than card $Y$. It remains to define the composite of the 2-cell $\alpha_{Y}: t_{Y} \longrightarrow t_{Y}: 0 \longrightarrow Y$ with $f: Y \longrightarrow Z$; we take it to be $\alpha_{Z}: t_{Z} \longrightarrow t_{Z}: 0 \longrightarrow Z$ if $\alpha<\operatorname{card} Z$, but to be $1: t_{Z} \longrightarrow t_{Z}: 0 \longrightarrow Z$ otherwise. Clearly $\mathcal{C}$ is now a 2-category. Let $\mathcal{A}$ be a small subcategory of $\mathcal{C}$ that is a candidate for density; by Theorem 5.13 we may suppose without loss of generality that $0 \in \mathcal{A}$. Let $\beta=\sup \{\operatorname{card} A \mid A \in \mathcal{A}\}$, and choose an $X \in \mathcal{C}$ with $\beta<\operatorname{card} X$. We exhibit a 2-natural transformation $\phi=\left(\phi_{A}: \mathcal{C}(A, X) \rightarrow \mathcal{C}(A, 1)\right)_{A \in \mathcal{A}}$ not induced by the unique map $X \longrightarrow 1$, showing by (5.5) that $\mathcal{A}$ is not dense in $\mathcal{C}$.

For $A \neq 0$, we are forced to take for $\phi_{A}$ the unique functor into $\mathcal{C}(A, 1)=1$; while $\phi_{0}$ is to be some monoid-map $\psi: \mathcal{C}(0, X) \rightarrow \mathcal{C}(0,1)$. For any choice of $\psi$, the naturality of $\phi$ (as distinct from its 2-naturality) is automatic. The only non-trivial 2-naturality conditions involve the 2-cells $\alpha_{A}: t_{A} \longrightarrow t_{A}: 0 \longrightarrow A$ for $A \in \mathcal{A}$; such a condition evaluated at $f \in \mathcal{C}(A, X)$ gives $\psi\left(\alpha_{X}\right)=1$ if $\alpha>0$ and $\psi\left(0_{X}\right)=0_{1}$; and hence $\phi$ is 2-natural if $\psi\left(\alpha_{X}\right)$ is so given for each $\alpha<\beta$. We are at liberty to choose $\psi$ arbitrarily on the other generators of $\mathcal{C}(0, X)$, and in particular on $\beta_{X}$. If we choose $\psi\left(\beta_{X}\right)$ to be not 1 but $0_{1}$, the 2 -natural $\phi$ is not induced by $X \longrightarrow 1$.

\subsection{Presentations of the density of a fully-faithful $K$ in terms of $K$ - absolute colimits}

In this section we use the results of 4.10 above to discuss, in terms of colimits in $\mathcal{C}$, the density of a fully faithful $K: \mathcal{A} \longrightarrow \mathcal{C}$.

Let $F: \mathcal{L}^{\text {op }} \longrightarrow \mathcal{V}$ and $P: \mathcal{L} \longrightarrow \mathcal{C}$. If the colimit $F \star P$ exists in $\mathcal{C}$ and is preserved by $\widetilde{K}: \mathcal{C} \longrightarrow\left[\mathcal{A}^{\text {op }}, \mathcal{V}\right]$, we shall call it a $K$-absolute colimit in $\mathcal{C}$. If $\mathcal{L}$ is small, it follows from 3.3 that $K$-absoluteness of the colimit $F \star P$ is equivalent to its preservation by $\mathcal{C}(K A,-): \mathcal{C} \longrightarrow \mathcal{V}$ for each $A \in \mathcal{A}$; and in any case preservation by these representables implies $K$-absoluteness. It is immediate from the definition in 4.10 of $K$-cylinder that:

Lemma 5.18 The unit $\alpha: F \longrightarrow \mathcal{C}(P-, F \star P)$ of any $K$-absolute colimit in $\mathcal{C}$ is a $K$ cylinder.

In proving Theorem 4.93 we observed that $1: \widetilde{K} C \longrightarrow \mathcal{C}(K-, C)$ is always a $K$-cylinder. Indeed, since the colimit $\widetilde{K} C \cong \widetilde{K} C \star Y$ is pointwise by $(3.10)$, this cylinder is sent to a colimit-cylinder not only by $\widetilde{K}$ but by each $\mathcal{C}(K A,-)$ : which for large $\mathcal{A}$ is in general a stronger assertion.

Theorem 5.19 For a fully-faithful $K: \mathcal{A} \longrightarrow \mathcal{C}$ the following are equivalent:

(i) $K$ is dense.

(ii) Every $K$-cylinder $\alpha: F \longrightarrow \mathcal{C}(P-, C)$ is the unit of a $K$-absolute colimit $F \star P \cong C$.

(iii) For each $C \in \mathcal{C}$, the identity $1: \widetilde{K} C \longrightarrow \mathcal{C}(K-, C)$ as unit exhibits $C$ as $\widetilde{K} C \star K$, and this colimit is $K$-absolute, preserved in fact by the representables $\mathcal{C}(K A,-): \mathcal{C} \longrightarrow \mathcal{V}$. 
(iv) Each $C \in \mathcal{C}$ is a $K$-absolute colimit of objects of $\mathcal{A}$, in the sense that $C$ is the value of a $K$-absolute colimit of the form $F \star K Q$.

(v) There is a family $\Phi=\left(F_{\gamma}: \mathcal{L}_{\gamma}^{\mathrm{op}} \longrightarrow \mathcal{V}, P_{\gamma}: \mathcal{L}_{\gamma} \longrightarrow \mathcal{C}\right)_{\gamma \in \Gamma}$ of diagrams in $\mathcal{C}$ such that each colimit $F_{\gamma} \star P_{\gamma}$ exists and is $K$-absolute, and such that $\mathcal{C}$ is the closure of $\mathcal{A}$ under this family of colimits.

If $\mathcal{A}$ is small, the colimits in (iv) and (v) may be taken to be small.

Proof. (i) implies (ii) since, when $\widetilde{K}$ is fully faithful, it reflects colimits by 3.5. By the remark preceding the theorem, (ii) gives (iii) as a special case. Then (iii) gives (iv) by taking $F=\widetilde{K} C$ and $Q=1$; and the domain of $F$ here is $\mathcal{A}$, so that the colimit is small if $\mathcal{A}$ is. From (iv) we get (v) by taking for $\Phi$ the one-object family $(F, K Q)$. Finally, by Theorem 4.98 and Lemma 5.4, it follows from (v) that $1: K \rightarrow K$ exhibits $1_{\mathcal{C}}$ as $\operatorname{Lan}_{K} K$, giving (i) by Theorem $5.1(\mathrm{v})$.

The chief purpose of this theorem is to exhibit (v) as a practical criterion for the density of $K$. We shall say that the density of $\mathcal{A}$ in $\mathcal{C}$ is presented (or exhibited) by the family $\Phi=\left(F_{\gamma}, P_{\gamma}\right)_{\gamma \in \Gamma}$; or, more loosely, by the family $F_{\gamma} \star P_{\gamma}$ of $K$-absolute colimits. We may call such a family a presentation of the density of $\mathcal{A}$ in $\mathcal{C}$, or simple a density presentation. We give a few examples of density exhibited by such a presentation.

Proposition 5.20 Given full subcategories $\mathcal{A} \subset \mathcal{D} \subset \mathcal{C}$, if every $D \in \mathcal{D}$ is a retract in $\mathcal{D}_{0}$ of some $A \in \mathcal{A}$, and if $\mathcal{D}$ is dense in $\mathcal{C}$, then $\mathcal{A}$ is dense in $\mathcal{C}$.

Proof. Given $D \in \mathcal{D}$ there is an $A \in \mathcal{A}$ with $i: D \longrightarrow A$ and $r: A \longrightarrow D$ such that $r i=1$. In these circumstances

$$
A \underset{1}{\stackrel{i r}{\longrightarrow}} A \underset{r}{\longrightarrow} D
$$

is a coequalizer in the ordinary category $\mathcal{C}_{0}$, and is moreover one preserved by any functor whatsoever. Being in particular preserved by the representables $\mathcal{C}(C,-)_{0}: \mathcal{C}_{0} \longrightarrow \mathcal{V}_{0}$, it is by 3.8 in fact a coequalizer in $\mathcal{C}$; and then, being preserved by the representables $\mathcal{C}(K A,-): \mathcal{C} \longrightarrow \mathcal{V}$, where $K: \mathcal{A} \longrightarrow \mathcal{C}$ is the inclusion, it is a $K$-absolute colimit. On the other hand, if $J: \mathcal{D} \longrightarrow \mathcal{C}$ is the inclusion, every $C \in \mathcal{C}$ is the colimit $\widetilde{J} C \star J$ of objects of $\mathcal{D}$; and by Theorem 5.19 (iii) this colimit is preserved by each representable $\mathcal{C}(J D,-)$, and so a fortiori by each $\mathcal{C}(K A,-)$; whence it, too, is $K$-absolute. Since $\mathcal{C}$ is therefore the closure of $\mathcal{A}$ under the $K$-absolute colimits of the two types (5.21) and $\widetilde{J} C \star J$, these constitute a presentation exhibiting $\mathcal{A}$ as dense in $\mathcal{C}$.

A category with a small dense subcategory does not in general admit a one-object full subcategory that is dense; for instance, in Set $\times$ Set, no single object can even be a generator. However:

Proposition 5.22 Let $\mathcal{C}$ have the small dense subcategory $\mathcal{G}$, and let $H=\sum_{G \in \mathcal{G}} G$ exist in $\mathcal{C}$. Then $H$ is dense in $\mathcal{C}$ provided that no $\mathcal{G}_{0}\left(G, G^{\prime}\right)$ is empty. 
Proof. Because of the last proviso, every $G \in \mathcal{G}$ is a retract in $\mathcal{C}_{0}$ of $H$. Since $G$ is dense in $\mathcal{C}$, so too by Theorem 5.13 is $\mathcal{G} \cup\{H\}$. The density of $H$ now follows from Proposition 5.20 .

Proposition 5.23 Let $\mathcal{C}$ have a full subcategory $\mathcal{A}$, and an initial object 0 such that $\mathcal{C}(A, 0)=0$ for each $A \in \mathcal{A}$. Then if $\mathcal{A} \cup\{0\}$ is dense in $\mathcal{C}$, so is $\mathcal{A}$.

Proof. $\quad 0 \in \mathcal{C}$ is the colimit of the empty diagram, and by hypothesis it is $K$-absolute where $K: \mathcal{A} \longrightarrow \mathcal{C}$ is the inclusion. The proof concludes like that of Proposition 5.20.

Another example of a density presentation comes at once from the examples following Theorem 4.91 and Theorem 4.98:

Proposition 5.24 In the case $\mathcal{V}=$ Set, let the small set $\left\{G_{x}\right\}_{x \in X}$ of objects constitute a regular generator of the cocomplete category $\mathcal{C}$. Then the full subcategory given by the small coproducts of the $G_{x}$ is dense in $\mathcal{C}$; and if the $G_{x}$ are all finitely presentable, we can replace "small" here by "finite".

For example, if $\mathcal{C}$ is an equational category of algebras (with finitary operations), the free algebra $F 1$ on one generator is easily seen to be a regular generator and to be finitely presentable. We conclude by Proposition 5.24 that the free algebras $F m$ with $m$ finite constitute a small dense subcategory. Here, however, we have already noted in 5.3 Isbell's result that, if all the basic operations have arity $\leq n$, then $F n$ alone is dense in $\mathcal{C}$.

Further examples of density presentations will be given in $5.7-5.10$ below.

\subsection{The characterization of functor categories $\left[\mathcal{A}^{\text {op }}, \mathcal{V}\right]$; small projectives and Cauchy completion; Morita equivalence}

We now apply some of the results above to the characterization of $\mathcal{V}$-categories of the form $\left[\mathcal{A}^{\mathrm{op}}, \mathcal{V}\right]$ for some small $\mathcal{A}$.

We shall say that an object $A$ in a cocomplete $\mathcal{V}$-category $\mathcal{C}$ is small-projective if the representable $\mathcal{C}(A,-): \mathcal{C} \longrightarrow \mathcal{V}$ preserves all small colimits. We record for future use:

Proposition 5.25 A retract $D$ of a small-projective $A$ in a cocomplete $\mathcal{C}$ is again smallprojective.

Proof. Let $F \star G$ be a small colimit in $\mathcal{C}$. We have the coequalizer (5.21) which is preserved by any functor (and hence sent to an equalizer by a contravariant functor). So we have a commutative diagram

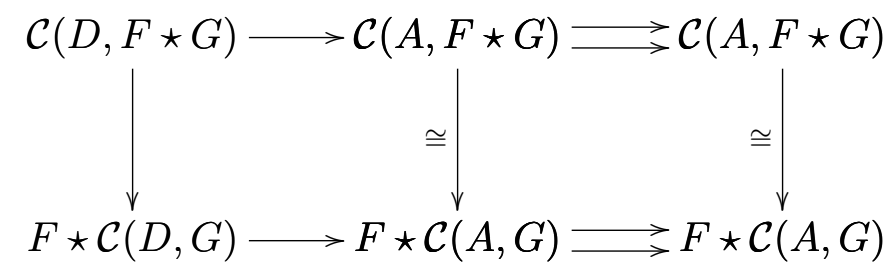

where the rows are equalizer diagrams and the marked comparison maps are isomorphisms; whence the remaining comparison map is an isomorphism too. 
Theorem 5.26 In order that $\mathcal{C}$ be equivalent to $\left[\mathcal{A}^{\mathrm{op}}, \mathcal{V}\right]$ for some small $\mathcal{A}$, it is necessary and sufficient that $\mathcal{C}$ be cocomplete and that there be a small set of small-projective objects in $\mathcal{C}$ constituting a strong generator for $\mathcal{C}$.

Proof. We have seen that $\left[\mathcal{A}^{\mathrm{op}}, \mathcal{V}\right]$ is cocomplete, and that the small full subcategory $\mathcal{A}$ of the representables is dense in $\left[\mathcal{A}^{\mathrm{op}}, \mathcal{V}\right]$, and thus a fortiori strongly generating. Moreover the representable $\mathcal{A}(-, A)$ is small-projective; for $\left[\mathcal{A}^{\mathrm{op}}, \mathcal{V}\right](\mathcal{A}(-, A)$, ?) is isomorphic by Yoneda to the evaluation $E_{A}:\left[\mathcal{A}^{\mathrm{op}}, \mathcal{V}\right] \longrightarrow \mathcal{V}$, which preserves all small colimits by 3.3 . This proves the necessity.

For the sufficiency, let $K: \mathcal{G} \longrightarrow \mathcal{C}$ be the inclusion of the full subcategory determined by the small set of objects in question. Since $K$ is strongly generating, $\widetilde{K}: \mathcal{C} \longrightarrow\left[\mathcal{G}^{\text {op }}, \mathcal{V}\right]$ is conservative. Moreover $\widetilde{K}$ preserves all small colimits, since each $\mathcal{C}(K G,-)$ does so. In particular it preserves for each $C \in \mathcal{C}$ the colimit $\widetilde{K} C \star K$. Since, by the remarks following Lemma $5.4, \widetilde{K}$ sends $1: \widetilde{K} C \longrightarrow \mathcal{C}(K-, C)$ to a colimit cylinder, we conclude by the observations at the beginning of 3.6 that 1 is already a colimit cylinder, exhibiting $C$ as $\widetilde{K} C \star K$. Thus $K$ is dense by Theorem $5.1(\mathrm{iv})$; and consequently $\widetilde{K}$ is fully faithful.

To prove $\widetilde{K}$ an equivalence, it remains by 1.11 to prove it essentially surjective. Given $F \in\left[\mathcal{A}^{\text {op }}, \mathcal{V}\right]$, the colimit $F \star K$ exists because $\mathcal{A}$ is small and $\mathcal{C}$ is cocomplete; and since $\widetilde{K}$ preserves small colimits we have $\widetilde{K}(F \star K) \cong F \star \widetilde{K} K \cong F \star Y \cong F$.

There may well be various inequivalent $\mathcal{A}$ for which $\mathcal{C}$ is equivalent to $\left[\mathcal{A}^{\text {op }}, \mathcal{V}\right]$. If $\overline{\mathcal{A}}$ denotes the full subcategory of $\left[\mathcal{A}^{\text {op }}, \mathcal{V}\right]$ determined by the small-projectives, then $\mathcal{A} \subset \overline{\mathcal{A}}$, and the proof above gives $\left[\mathcal{A}^{\mathrm{op}}, \mathcal{V}\right] \simeq\left[\overline{\mathcal{A}}^{\mathrm{op}}, \mathcal{V}\right]$ if $\overline{\mathcal{A}}$ is small. In general $\overline{\mathcal{A}}$ is strictly bigger than $\mathcal{A}$; and for many $\mathcal{V}$ it is small whenever $\mathcal{A}$ is. We shall show that in 5.8 that, for $\mathcal{V}=\operatorname{Set}, \overline{\mathcal{A}}$ consists of the retracts of the representables. The same is true for $\mathcal{V}=\mathrm{Cat}$ or CGTop; when $\mathcal{V}=\mathbf{A b}, \overline{\mathcal{A}}$ consists of the retracts of finite coproducts of representables; when $\mathcal{V}=\overline{\mathbb{R}}_{+}$, Lawvere [54] has shown $\overline{\mathcal{A}}$ to be the Cauchy completion of the (generalized) metric space $\mathcal{A}$. In all these cases $\overline{\mathcal{A}}$ is small when $\mathcal{A}$ is.

If $\mathcal{V}$ is such that this last is so, we may call $\overline{\mathcal{A}}$ the Cauchy completion of $\mathcal{A}$. The passage from $\mathcal{A}$ to $\overline{\mathcal{A}}$ is a closure operation; for $\overline{\mathcal{A}}$, as the small-projectives in $\left[\overline{\mathcal{A}}^{\mathrm{op}}, \mathcal{V}\right] \simeq\left[\mathcal{A}^{\mathrm{op}}, \mathcal{V}\right]$, is equivalent to $\overline{\mathcal{A}}$. Then $\mathcal{A}$ is Cauchy complete if the inclusion $\mathcal{A} \longrightarrow \overline{\mathcal{A}}$ is an equivalence. In these circumstances it follows at once from the facts above that, for small $\mathcal{A}$ and $\mathcal{B}$, we have $\left[\mathcal{A}^{\text {op }}, \mathcal{V}\right] \simeq\left[B^{\text {op }}, \mathcal{V}\right]$ if and only if $\overline{\mathcal{A}} \simeq \overline{\mathcal{B}}$. This relation is called Morita equivalence between $\mathcal{A}$ and $\mathcal{B}$; it was first studied by Morita [65] (see also [2]) in the case $\mathcal{V}=\mathrm{Ab}$ with $\mathcal{A}$ and $\mathcal{B}$ one-object $\mathbf{A b}$-categories - that is, rings $R$ and $S$; in which case $\left[\mathcal{A}^{\text {op }}, \mathcal{V}\right]$ and $\left[\mathcal{B}^{\text {op }}, \mathcal{V}\right]$ are the categories of right $R$-modules and right $S$-modules. The general situation has been studied by various authors ([66], [68], [27], [33]), and may be expressed in terms of the profunctors or distributors introduced independently by Lawvere [53] and Bénabou [8]; a profunctor from $\mathcal{A}$ to $\mathcal{B}$ is a functor $T: \mathcal{B} \otimes \mathcal{A}^{\mathrm{op}} \longrightarrow \mathcal{V}$, and these compose by the operation $S(C,-) \star T(-, A)$ to form a biclosed bicategory.

For some $\mathcal{V}$, however, $\overline{\mathcal{A}}$ may be large when $\mathcal{A}$ is small. An example is the category $\mathcal{V}_{0}$ of complete lattices and sup-preserving maps, with the tensor product such that the maps $A \otimes B \longrightarrow C$ are the functions $A \times B \longrightarrow C$ which are sup-preserving in each variable separately. Here the Cauchy completion of the unit $\mathcal{V}$-category $\mathcal{I}$ is not small, containing 
for each set $X$ the lattice $\mathcal{P} X$ of subsets of $X$. For such $\mathcal{V}$ the relation between $\left[\mathcal{A}^{\text {op }}, \mathcal{V}\right]$ and $\left[\overline{\mathcal{A}}^{\mathrm{op}}, \mathcal{V}\right]$ is more complicated, and has been given by Lindner [55]:

Theorem 5.27 For any $\mathcal{V}$ and any small $\mathcal{V}$-category $\mathcal{A}$, let $\overline{\mathcal{A}}$ consist of the smallprojectives in $\left[\mathcal{A}^{\mathrm{op}}, \mathcal{V}\right]$. Then if $K: \overline{\mathcal{A}} \longrightarrow\left[\mathcal{A}^{\mathrm{op}}, \mathcal{V}\right]$ is the inclusion,

$$
\widetilde{K}:\left[\mathcal{A}^{\mathrm{op}}, \mathcal{V}\right] \longrightarrow\left[\overline{\mathcal{A}}^{\mathrm{op}}, \mathcal{V}\right]
$$

induces an equivalence of $\left[\mathcal{A}^{\mathrm{op}}, \mathcal{V}\right]$ with the full subcategory $\operatorname{Acc}\left[\overline{\mathcal{A}}^{\mathrm{op}}, \mathcal{V}\right]$ determined by the accessible functors; and the inverse to this equivalence is composition with the inclusion $Z: \mathcal{A} \longrightarrow \overline{\mathcal{A}}$.

Proof. Since $K Z=Y: \mathcal{A} \longrightarrow\left[\mathcal{A}^{\text {op }}, \mathcal{V}\right]$ is dense, the fully-faithful $K$ is dense by Theorem 5.13. Hence $\widetilde{K}:\left[\mathcal{A}^{\mathrm{op}}, \mathcal{V}\right] \longrightarrow\left[\overline{\mathcal{A}}^{\mathrm{op}}, \mathcal{V}\right]$ is fully faithful, and it remains to determine its full image. For any $F: \mathcal{A}^{\text {op }} \longrightarrow \mathcal{V}$ we have $\widetilde{K} F=\left[\mathcal{A}^{\text {op }}, \mathcal{V}\right](K-, F)$, which by the Yoneda (3.17) is $\left[\mathcal{A}^{\mathrm{op}}, \mathcal{V}\right](K-, F \star Y)$. Since each $K B$ is small-projective, this is isomorphic to the colimit $F ? \star\left[\mathcal{A}^{\mathrm{op}}, \mathcal{V}\right](K-, Y ?)=F ? \star\left[\mathcal{A}^{\mathrm{op}}, \mathcal{V}\right](K-, K Z ?) \cong F ? \star \overline{\mathcal{A}}(-, Z ?)$. The last term here is a small colimit of representables $\overline{\mathcal{A}}^{\text {op }} \longrightarrow \mathcal{V}$, and hence is accessible by Proposition 4.83. Moreover composing it with $Z$ gives $F ? \star \overline{\mathcal{A}}(Z-, Z$ ?) $\cong F ? \star \mathcal{A}(-, ?) \cong F$, verifying that composition with $Z$ is the inverse. To show finally that every accessible is in the image of $\widetilde{K}$, it suffices to show that every representable $\overline{\mathcal{A}}(-, B)$ is in this image, since $\widetilde{K}$ preserves small colimits. Composing this representable with $Z$ gives $\overline{\mathcal{A}}(Z-, B): \mathcal{A}^{\mathrm{op}} \longrightarrow \mathcal{V}$; but $\overline{\mathcal{A}}(Z-, B)=\left[\mathcal{A}^{\mathrm{op}}, \mathcal{V}\right](Y-, K B) \cong K B$. Now we have $\widetilde{K} K B=$ $\left[\mathcal{A}^{\mathrm{op}}, \mathcal{V}\right](K-, K B) \cong \overline{\mathcal{A}}(-, B)$, as desired.

This gives at once:

Proposition 5.28 For any $\mathcal{V}$ and any small $\mathcal{A}, \mathcal{B}$, we have an equivalence $\left[\mathcal{A}^{\mathrm{op}}, \mathcal{V}\right] \simeq$ $\left[\mathcal{B}^{\mathrm{op}}, \mathcal{V}\right]$ if and only if we have $\overline{\mathcal{A}} \simeq \overline{\mathcal{B}}$.

Of course, since $\operatorname{Acc}\left[\overline{\mathcal{A}}^{\text {op }}, \mathcal{V}\right]=\left[\overline{\mathcal{A}}^{\text {op }}, \mathcal{V}\right]$ when $\overline{\mathcal{A}}$ is small, these results include those derived more simply above for small $\overline{\mathcal{A}}$. (Another approach to Proposition 5.28 uses the observation that, if $\mathcal{B}$ is cocomplete in Theorem 4.21 , then $\widetilde{G}$ is cocontinuous if and only if each $G A$ is small-projective; whereupon $S F$ is small-projective for $F \in \overline{\mathcal{A}}$.)

\subsection{Existence and characterization of the left Kan extension along a fully- faithful dense functor in terms of a density presentation; adjoint- functor theorems involving density}

We return to the consideration of a fully-faithful $K: \mathcal{A} \longrightarrow \mathcal{C}$, now supposing it to be dense; and we use the results of 4.10 again, this time to discuss left Kan extensions along $K$. When we say of a functor $G: \mathcal{C} \longrightarrow \mathcal{B}$ that it preserves $K$-absolute colimits in $\mathcal{C}$, we mean only that it sends them to colimits in $\mathcal{B}$; the epithet " $K$-absolute" has in general no meaning when applied to colimits in $\mathcal{B}$. Since $K$ is fully faithful, we have by Proposition 4.23 that $S: \mathcal{C} \longrightarrow \mathcal{B}$ is of the form $\operatorname{Lan}_{K} G$ for some $G: \mathcal{A} \longrightarrow \mathcal{B}$ precisely when $S$ is canonically isomorphic to $\operatorname{Lan}_{K} S K$. 
Theorem 5.29 If $K: \mathcal{A} \longrightarrow \mathcal{C}$ is fully faithful and dense, the following properties of $S: \mathcal{C} \longrightarrow \mathcal{B}$ are equivalent:

(i) $1: S K \longrightarrow S K$ exhibits $S$ as $\operatorname{Lan}_{K} S K$.

(ii) $S$ preserves all $K$-absolute colimits.

(iii) For any density presentation $\Phi:\left(F_{\gamma}, P_{\gamma}\right)_{\gamma \in \Gamma}$ of $K: \mathcal{A} \longrightarrow \mathcal{C}$, each of the colimits $F_{\gamma} \star P_{\gamma}$ is preserved by $S$.

(iv) $S$ preserves the colimit $\widetilde{K} C \star K$ for each $C \in \mathcal{C}$.

(v) There is some density presentation $\Phi=\left(F_{\gamma}, P_{\gamma}\right)_{\gamma \in \Gamma}$ of $K: \mathcal{A} \longrightarrow \mathcal{C}$ for which $S$ preserves each of the colimits $F_{\gamma} \star P_{\gamma}$.

If $\mathcal{A}$ is small, it suffices in (ii) that $S$ preserve all small $K$-absolute colimits; and moreover it may then be supposed that the colimits in (v) are small.

Proof. (i) implies (ii) (including the modification when $\mathcal{A}$ is small) by Theorem 4.97 and Theorem 5.19(ii). Trivially (ii) implies (iii); and since $(\widetilde{K} C, K)$ is, by Theorem 5.19 , itself a density presentation, (iii) implies (iv) and (iv) implies (v). Finally (v) implies (i) by Theorem 4.98 and Lemma 5.4 .

As for the existence of $\operatorname{Lan}_{K} G$, a direct application of Theorem 4.98 gives:

Theorem 5.30 Let $\Phi=\left(F_{\gamma}, P_{\gamma}\right)_{\gamma \in \Gamma}$ be a density presentation of the fully-faithful and dense $K: \mathcal{A} \longrightarrow \mathcal{C}$, and let $\mathcal{B}$ admit all $F_{\gamma}$-indexed colimits for all $\gamma \in \Gamma$. Then every $G: \mathcal{A} \longrightarrow \mathcal{B}$ admits a left Kan extension $\operatorname{Lan}_{K} G: \mathcal{C} \longrightarrow \mathcal{B}$.

Recall the equivalence $[\mathcal{A}, \mathcal{B}]^{\prime} \simeq[\mathcal{C}, \mathcal{B}]^{l}$ of Theorem 4.99, where $[\mathcal{A}, \mathcal{B}]^{\prime}$ is the full subcategory given by those $G: \mathcal{A} \longrightarrow \mathcal{B}$ admitting left Kan extensions along $K$, and where $[\mathcal{C}, \mathcal{B}]^{l}$ is the full subcategory given by those $S: \mathcal{C} \longrightarrow \mathcal{B}$ of the form $\operatorname{Lan}_{K} G$ for some $G$ (necessarily isomorphic to $S K$ ). Where $K$ is dense, Theorem 5.29 now identifies such $S$ as those preserving all $K$-absolute colimits. We extract from this the most important practical case, writing $\operatorname{Cocts}^{K}[\mathcal{C}, \mathcal{B}]$ for the full subcategory of $[\mathcal{C}, \mathcal{B}]$ given by those $S: \mathcal{C} \longrightarrow \mathcal{B}$ which preserve all small $K$-absolute colimits:

Theorem 5.31 Let $\Phi=\left(F_{\gamma}, P_{\gamma}\right)_{\gamma \in \Gamma}$ be a density presentation of the fully-faithful and dense $K: \mathcal{A} \longrightarrow \mathcal{C}$, for which the domain $L_{\gamma}$ of each $F_{\gamma}$ is small; and let $\mathcal{B}$ admit all $F_{\gamma}$-indexed colimits for all $\gamma \in \Gamma$. Then we have an equivalence

$$
\operatorname{Lan}_{K}:[\mathcal{A}, \mathcal{B}] \simeq \operatorname{Cocts}^{K}[\mathcal{C}, \mathcal{B}]
$$

whose inverse is the restriction of $[K, 1]:[\mathcal{C}, \mathcal{B}] \longrightarrow[\mathcal{A}, \mathcal{B}]$. 
Note that, since the fully-faithful and dense $Y: \mathcal{A} \longrightarrow\left[\mathcal{A}^{\mathrm{op}}, \mathcal{V}\right]$ has $\tilde{Y}=1$, every colimit in $\left[\mathcal{A}^{\mathrm{op}}, \mathcal{V}\right]$ is $Y$-absolute. Thus Theorem 5.31 generalizes the assertion (4.56) of Theorem 4.51 .

We end this section with two adjoint-functor theorems involving small dense subcategories.

Theorem 5.32 Let $\mathcal{C}$ be complete and cocomplete, and let $\mathcal{A}$ be a small dense full subcategory whose inclusion $K: \mathcal{A} \longrightarrow \mathcal{C}$ has a density presentation $\Phi=\left(F_{\gamma}, P_{\gamma}\right)_{\gamma \in \Gamma}$. Let $\mathcal{B}$ be the closure under small colimits of a full subcategory $\mathcal{D}$. Let $T: \mathcal{C} \longrightarrow \mathcal{B}$ preserve the colimits $F_{\gamma} \star P_{\gamma}$, and let each $F_{\gamma} \star T P_{\gamma}$ be further preserved by the representables $\mathcal{B}(D,-)$ with $D \in \mathcal{D}$. Then $T$ has a left adjoint if it is continuous.

Proof. For each $D \in \mathcal{D}$, the functor $\mathcal{B}(D, T-): \mathcal{C} \longrightarrow \mathcal{V}$ preserves the colimits $F_{\gamma} \star$ $P_{\gamma}$, and hence by Theorem 5.29 is the left Kan extension of its restriction to $\mathcal{A}$. It is therefore accessible by Proposition 4.83; and since it is continuous, it is by Theorem 4.84 representable. That $T$ has a left adjoint now follows from Proposition 3.37.

A well-known special case of this theorem (see Satz 14.6 of [31]) is that where $\mathcal{V}=$ Set, where $\mathcal{C}$ and $\mathcal{B}$ are categories of finitary essentially-algebraic structures, and where $\mathcal{A}$ and $\mathcal{D}$ are the finitely-presentable objects in $\mathcal{C}$ and $\mathcal{B}$ respectively; the conclusion is then that a continuous $T$ has a left adjoint if it preserves filtered colimits.

The next theorem, although we place it here for comparison, is quite different in nature, and could have been proved immediately after Theorem 5.1. It is a variant of Theorem 4.90 (the Special Adjoint Functor Theorem) - or rather, of its dual. A small dense subcategory replaces the generator, and then the hypothesis on cointersections may be dropped; moreover the present theorem is valid for any $\mathcal{V}$.

Theorem 5.33 If the cocomplete $\mathcal{C}$ has a small dense subcategory $\mathcal{A}$, every cocontinuous $S: \mathcal{C} \longrightarrow \mathcal{B}$ has a right adjoint.

Proof. If $K: \mathcal{A} \longrightarrow \mathcal{C}$ is the inclusion, $\operatorname{Lan}_{S K} K$ exists because $\mathcal{A}$ is small and $\mathcal{C}$ is cocomplete. Since $\operatorname{Lan}_{K} K \cong 1_{\mathcal{C}}$ by Theorem 5.1, it follows from Theorem 4.47 that $\operatorname{Lan}_{S} 1_{\mathcal{C}}$ exists. Again, since $\mathcal{A}$ is small, the cocontinuous $S$ preserves the Kan extensions $\operatorname{Lan}_{K} K$ and $\operatorname{Lan}_{S K} K$; whence, by Theorem 4.47 again, it preserves $\operatorname{Lan}_{S} 1_{\mathcal{C}}$. That $S$ has a right adjoint now follows from Theorem 4.81 .

Note that a special case of this theorem appeared in Theorem 4.51.

\subsection{The free completion of $\mathcal{A}$ with respect to all colimits with indexing- type in $\mathcal{F}$}

We now describe the free cocompletion of a $\mathcal{V}$-category with respect to colimits of some given class.

First observe that:

Proposition 5.34 For any $\mathcal{V}$-category $\mathcal{A}$, the set of accessible functors $\mathcal{A}^{\mathrm{op}} \longrightarrow \mathcal{V}$ is closed under small colimits. 
Proof. $\quad$ Let $H: \mathcal{L}^{\text {op }} \longrightarrow \mathcal{V}$ and $P: \mathcal{L} \longrightarrow\left[\mathcal{A}^{\text {op }}, \mathcal{V}\right]$, where $\mathcal{L}$ is small and each $P L$ is accessible. By Proposition 4.83, each $P L$ is the left Kan extension of its restriction to some small full subcategory $\mathcal{C}_{L}$ of $\mathcal{A}^{\text {op }}$. The union $\mathcal{C}$ of the $\mathcal{C}_{L}$ for $L \in \mathcal{L}$ is still small, because $\mathcal{L}$ is small; write $K: \mathcal{C} \longrightarrow \mathcal{A}^{\text {op }}$ for the inclusion. Since $K$ is fully faithful, it follows from Theorem 4.47 that each $P L$ is the left Kan extension of its restriction $(P L) K$ to $\mathcal{C}$. Write $Q: \mathcal{L} \longrightarrow[\mathcal{C}, \mathcal{V}]$ for the composite of $P$ with $[K, 1]:\left[\mathcal{A}^{\mathrm{op}}, \mathcal{V}\right] \longrightarrow[\mathcal{C}, \mathcal{V}]$, so that $Q L=(P L) K$; then we have $P L \cong \operatorname{Lan}_{K} Q L$. Now (3.21), expressing the cocontinuity of $\star$ in its second variable, along with (3.16), justifies the calculation $(H \star P) A=H ? \star(P$ ? $) A \cong$ $H ? \star(\widetilde{K} A \star Q ?) \cong \widetilde{K} A \star(H \star Q)=\left(\operatorname{Lan}_{K}(H \star Q)\right) A$. So $H \star P \cong \operatorname{Lan}_{K}(H \star Q)$ is accessible by Theorem 4.83 .

In other words, the full subcategory $\operatorname{Acc}\left[\mathcal{A}^{\mathrm{op}}, \mathcal{V}\right]$ of $\left[\mathcal{A}^{\mathrm{op}}, \mathcal{V}\right]$ is closed under small colimits. Note from (4.41) that $\operatorname{Acc}\left[\mathcal{A}^{\mathrm{op}}, \mathcal{V}\right]$ is a $\mathcal{V}$-category, even if $\mathcal{A}$ is large.

Now let $\mathcal{F}$ be any set of functors with codomain $\mathcal{V}$, for each element $F: \mathcal{L} \longrightarrow \mathcal{V}$ of which the domain $\mathcal{L}$ is small; that is, $\mathcal{F}$ is a set of small indexing types. We say that $\mathcal{C}$ is $\mathcal{F}$-cocomplete if it admits all colimits indexed by the elements of $\mathcal{F}$, and that $S: \mathcal{C} \longrightarrow \mathcal{B}$ is $\mathcal{F}$-cocontinuous if it preserves all such colimits - which we may call $\mathcal{F}$-colimits. We write $\mathcal{F}$-Cocts $[\mathcal{C}, \mathcal{B}]$ for the full subcategory of $[\mathcal{C}, \mathcal{B}]$ determined by the $\mathcal{F}$-cocontinuous functors. In the following we regard $\mathcal{A}$ as embedded in $\left[\mathcal{A}^{\mathrm{op}}, \mathcal{V}\right]$ by Yoneda.

Theorem 5.35 For any set $\mathcal{F}$ of small indexing types and any $\mathcal{V}$-category $\mathcal{A}$, let $\overline{\mathcal{A}}$ be the closure of $\mathcal{A}$ in $\left[\mathcal{A}^{\mathrm{op}}, \mathcal{V}\right]$ under $\mathcal{F}$-colimits, and let $K: \mathcal{A} \longrightarrow \overline{\mathcal{A}}$ be the inclusion. Then $\overline{\mathcal{A}} \subset \operatorname{Acc}\left[\mathcal{A}^{\mathrm{op}}, \mathcal{V}\right]$, and is therefore a $\mathcal{V}$-category. When $\mathcal{A}$ is small and the set $\mathcal{F}$ is small, $\overline{\mathcal{A}}$ is small. $\overline{\mathcal{A}}$ is $\mathcal{F}$-cocomplete, and every $\mathcal{F}$-colimit in $\overline{\mathcal{A}}$ is $K$-absolute; the totality of these $\mathcal{F}$-colimits constitutes a density presentation of $K$. A functor $S: \overline{\mathcal{A}} \longrightarrow \mathcal{B}$ is of the form $\operatorname{Lan}_{K} S K$ precisely when it is $\mathcal{F}$-cocontinuous; and we have an equivalence

$$
\operatorname{Lan}_{K}:[\mathcal{A}, \mathcal{B}]^{\prime} \simeq \mathcal{F} \text {-Cocts }[\overline{\mathcal{A}}, \mathcal{B}]
$$

whose inverse is the restriction of $[K, 1]$, where $[\mathcal{A}, \mathcal{B}]^{\prime}$ is the full subcategory of $[\mathcal{A}, \mathcal{B}]$ consisting of those $G$ for which $\operatorname{Lan}_{K} G$ exists. If $\mathcal{B}$ is $\mathcal{F}$-cocomplete, this is all of $[\mathcal{A}, \mathcal{B}]$, and the equivalence becomes

$$
\operatorname{Lan}_{K}:[\mathcal{A}, \mathcal{B}] \simeq \mathcal{F}-\operatorname{Cocts}[\overline{\mathcal{A}}, \mathcal{B}]
$$

exhibiting $\overline{\mathcal{A}}$ as "the free $\mathcal{F}$-cocompletion of $\mathcal{A}$ ".

Proof. That $\overline{\mathcal{A}} \subset \operatorname{Acc}\left[\mathcal{A}^{\text {op }}, \mathcal{V}\right]$ follows from the transfinite construction of $\overline{\mathcal{A}}$ in 3.5 , along with Proposition 5.34. That $\overline{\mathcal{A}}$ is small when $\mathcal{A}$ and $\mathcal{F}$ are small was observed in 3.5. $\overline{\mathcal{A}}$ is $\mathcal{F}$-cocomplete by its construction, since $\mathcal{F}$-colimits certainly exist in $\left[\mathcal{A}^{\text {op }}, \mathcal{V}\right]$, each element of $\mathcal{F}$ being small; and these $\mathcal{F}$-colimits in $\overline{\mathcal{A}}$ are $K$-absolute, being preserved by construction under the inclusion $\overline{\mathcal{A}} \longrightarrow\left[\mathcal{A}^{\mathrm{op}}, \mathcal{V}\right]$, which is $\widetilde{K}$ by Proposition 5.16 . The $\mathcal{F}$-colimits in $\overline{\mathcal{A}}$ form a density presentation for $K$ by the definition of this following Theorem 5.19; and then the characterisation of the $S$ of the form $\operatorname{Lan}_{K} S K$ follows from Theorem 5.29. The first equivalence displayed comes from Theorem 4.99, and the identification of $[\mathcal{A}, \mathcal{B}]^{\prime}$ with $[\mathcal{A}, \mathcal{B}]$ when $\mathcal{B}$ is $\mathcal{F}$-cocomplete comes from Theorem 5.30. 
Note that the second equivalence of Theorem 5.35 is a special case of that of Theorem 5.31; what is new here is the freeness of the $\mathcal{F}$-cocompletion, which allows us to replace $\operatorname{Cocts}^{K}[\mathcal{C}, \mathcal{B}]$ by $\mathcal{F}$-Cocts $[\mathcal{C}, \mathcal{B}]$. (Often, using the word "completion" in a wider sense, we may refer to the $\mathcal{F}$-cocompletion of $\mathcal{A}$ as the completion of $\mathcal{A}$ under $\mathcal{F}$-colimits.)

As the first example of such an $\mathcal{F}$-cocompletion, take $\mathcal{F}$ to consist of all small indexing types, so that $\overline{\mathcal{A}}$ is simply the cocompletion of $\mathcal{A}$. From Proposition 4.83 and Proposition 5.34, this cocompletion is precisely the $\mathcal{V}$-category $\operatorname{Acc}\left[\mathcal{A}^{\mathrm{op}}, \mathcal{V}\right]$. When $\mathcal{A}$ is small, this reduces to $\left[\mathcal{A}^{\text {op }}, \mathcal{V}\right]$, and we re-find (part of) Theorem 4.51.

Other examples with $\mathcal{V} \neq$ Set will be found in the article [45] on enriched essentiallyalgebraic structures. For the remaining examples discussed below we take $\mathcal{V}=$ Set. The colimits we are interested in will then be conical ones, so that $\mathcal{F}$ is in effect a set of small categories.

Before we proceed, however, we anticipate a possible misunderstanding that may arise from the very special nature of our main examples. In the transfinite construction of 3.5 for the closure $\overline{\mathcal{A}}$ of $\mathcal{A}$ under $\mathcal{F}$-colimits in $\left[\mathcal{A}^{\mathrm{op}}, \mathcal{V}\right]$, the first step consists in taking all the $\mathcal{F}$-colimits in $\left[\mathcal{A}^{\mathrm{op}}, \mathcal{V}\right]$ of the objects of $\mathcal{A}$. In some important cases the full subcategory given by these $\mathcal{F}$-colimits is itself closed under $\mathcal{F}$-colimits and hence constitutes $\overline{\mathcal{A}}$; the construction then terminates after one step. This was so in the example above, where $\mathcal{F}$ consisted of all small indexing types; and it will also be so in the three important examples of 5.8-5.10 below. However, it is far from true in general. For instance, taking $\mathcal{V}=$ Set henceforth, if $\mathcal{F}$ consists of two discrete categories 0 and 2 , so that $\overline{\mathcal{A}}$ is the closure of $\mathcal{A}$ under finite coproducts, we reach $\overline{\mathcal{A}}$ only after $\omega$ steps of the iteration. Less trivially, if $\mathcal{F}$ consists of the single category $0 \Longrightarrow 1$, so that $\overline{\mathcal{A}}$ is the closure of $\mathcal{A}$ under coequalizers, consider the case where $\mathcal{A}$ has two objects $A, B$ and three non identity maps $f, g, h: A \longrightarrow B$. In the first step we add in the coequalizers $P, Q, R$ of the respective pairs $(f, g),(f, h)$, and $(g, h)$; but now there are two maps $A \longrightarrow P$, and we must next add in the coequalizer of these, and so on.

\subsection{Cauchy completion as the idempotent-splitting completion when $\mathcal{V}=$ Set}

For our first main example with $\mathcal{V}=$ Set, let $\mathcal{F}$ consist of the single category with one object and with one non-identity endomorphism $e$ satisfying $e^{2}=e$. Then a diagram of type $\mathcal{F}$ in $\mathcal{A}$ is an object $A$ with an idempotent endomorphism $e$. This diagram has a colimit $r: A \longrightarrow B$ in $\mathcal{A}$ if and only if the idempotent $e$ splits; that is, $e$ has the form $e=i r$ for maps $r: A \longrightarrow B$ and $i: B \longrightarrow A$ with $r i=1$. Such a splitting is unique (to within isomorphism) if it exists; so that the split idempotents correspond bijectively to the retracts of $A$ in $\mathcal{A}$. In this case $\overline{\mathcal{A}}$, which we may call the idempotent-splitting completion of $\mathcal{A}$, clearly consists of all the retracts in $\left[\mathcal{A}^{\mathrm{op}}\right.$, Set $]$ of the representables $\mathcal{A}(-, A) \in \mathcal{A}$; it is evidently small if $\mathcal{A}$ is. Moreover $\mathcal{B}$ is $\mathcal{F}$-complete precisely when all idempotents split in $\mathcal{B}$. In fact we have the result promised in 5.5 above:

Theorem 5.36 For a small $\mathcal{A}$, the small projectives in $\left[\mathcal{A}^{\mathrm{op}}\right.$, Set] are precisely the re- 
tracts of the representables; so that the idempotent-splitting completion of $\mathcal{A}$ is its Cauchy completion. Moreover $\mathcal{A}$ is Cauchy complete exactly when all the idempotents split in $\mathcal{A}$.

Proof. We saw in the proof of Theorem 5.26 that the representables in $\left[\mathcal{A}^{\text {op }}\right.$, Set] are small-projective, whence so too are their retracts by Proposition 5.25. Suppose conversely that $F: \mathcal{A}^{\mathrm{op}} \longrightarrow$ Set is small-projective. Then $\left[\mathcal{A}^{\mathrm{op}}, \operatorname{Set}\right](F,-)$ preserves all small colimits, and in particular the canonical expression (3.32) of $F$ as a colimit of representables. Thus $\left[\mathcal{A}^{\mathrm{op}}, \operatorname{Set}\right](F, F)$ is the canonical colimit, indexed by $(\mathrm{el} F)^{\mathrm{op}}$, of the sets $\left[\mathcal{A}^{\mathrm{op}}, \operatorname{Set}\right](F, \mathcal{A}(-, A))$. Since colimits in Set are calculated by (3.35), the element $1_{F}$ of $\left[\mathcal{A}^{\mathrm{op}}\right.$, Set $](F, F)$ is the composite of some $r: \mathcal{A}(-, A) \longrightarrow F$ in el $F$ with some $i: F \longrightarrow \mathcal{A}(-, A)$; that is, $F$ is a retract of some $\mathcal{A}(-, A)$.

To say that $\mathcal{A}$ is Cauchy complete is, by 5.5 , to say that the inclusion $\mathcal{A} \longrightarrow \overline{\mathcal{A}}$ is an equivalence, or that every object of $\overline{\mathcal{A}}$ is isomorphic to one in $\mathcal{A}$. Since idempotents split uniquely if at all, this is exactly to say that all idempotents split in $\mathcal{A}$.

Note that, in this example, the inclusion $\mathcal{A} \longrightarrow \overline{\mathcal{A}}$ preserves such $\mathcal{F}$-colimits as exist in $\mathcal{A}$. In fact $\mathcal{F}$-colimits for this $\mathcal{F}$ are absolute - they are preserved by any functor whatsoever. This example is quite atypical, however: for a general $\mathcal{F}$ the inclusion $\mathcal{A} \longrightarrow \overline{\mathcal{A}}$ by no means preserves the $\mathcal{F}$-colimits that exist in $\mathcal{A}$. For instance, consider the completion of $\mathcal{A}$ under finite coproducts: $\mathcal{A}(C, A+B)$ is in general quite different from $\mathcal{A}(C, A)+\mathcal{A}(C, B)$.

\subsection{The finite-colimit completion when $\mathcal{V}=$ Set}

Our next example with $\mathcal{V}=$ Set is the completion of $\mathcal{A}$ under finite colimits; since the number of finite categories is small, the finite completion of $\mathcal{A}$ is small if $\mathcal{A}$ is.

Theorem 5.37 The completion of $\mathcal{A}$ under finite colimits is the full subcategory of $\left[\mathcal{A}^{\mathrm{op}}\right.$, Set $]$ determined by all finite colimits of the representables.

Proof. $\quad$ Let $\overline{\mathcal{A}} \subset\left[\mathcal{A}^{\text {op }}\right.$, Set $]$ consist of all finite colimits of the representables; we are to show that $\overline{\mathcal{A}}$ is closed under finite colimits. Clearly $\overline{\mathcal{A}}$ contains the initial object of $\left[\mathcal{A}^{\text {op }}\right.$, Set $]$. Consider objects $F=\operatorname{colim} Y T$ and $G=\operatorname{colim} Y S$ of $\overline{\mathcal{A}}$, where $T: \mathcal{P} \longrightarrow \mathcal{A}$ and $F: \mathcal{Q} \longrightarrow \mathcal{A}$ with $\mathcal{P}$ and $\mathcal{Q}$ finite. Let $R: \mathcal{P}+\mathcal{Q} \longrightarrow \mathcal{A}$ be the functor with components $T$ and $S$. Since an inductive cone over $Y R$ with any vertex $H$ clearly consists of independent cones over $Y T$ and $Y S$, it follows that colim $Y R=F+G$. Hence $\overline{\mathcal{A}}$ is closed under finite coproducts, and it remains to prove it closed under finite coequalizers.

With $F$ and $G$ as above, let the colimit cones be $\left(\rho_{P}: \mathcal{A}(-, T P) \longrightarrow F\right)$ and $\left(\sigma_{Q}: \mathcal{A}(-, S Q) \longrightarrow G\right)$, and consider $\alpha, \beta: F \longrightarrow G$. Since the $\rho_{P}$ are jointly epimorphic, a map $\gamma: G \longrightarrow H$ satisfies $\gamma \alpha=\gamma \beta$ if and only if it satisfies $\gamma \alpha \rho_{P}=\gamma \beta \rho_{P}$ for each $P \in \mathcal{P}$. The map $\alpha \rho_{P}: \mathcal{A}(-, T P) \longrightarrow G$ corresponds by Yoneda to an element of $G T P$. Since the $\sigma_{Q, T P}: \mathcal{A}(T P, S Q) \longrightarrow G T P$ are jointly surjective in Set, this element is the image under $\sigma_{P^{\prime}, T P}$ of some $u_{P} \in \mathcal{A}\left(T P, S P^{\prime}\right)$ for some $P^{\prime} \in \mathcal{Q}$. By the naturality of Yoneda we then 
have commutativity in the left diagram of
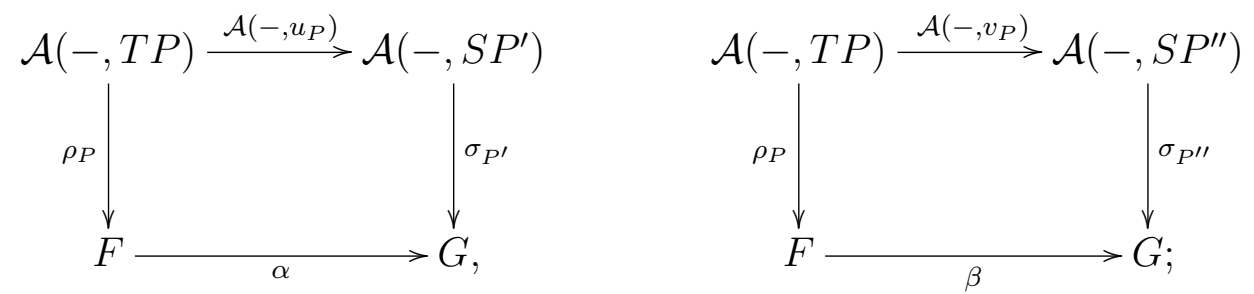

while the corresponding right diagram comes from a similar argument applied to $\beta$. The condition $\gamma \alpha \rho_{P}=\gamma \beta \rho_{P}$ now becomes $\gamma \sigma_{P^{\prime}} \mathcal{A}\left(-, u_{P}\right)=\gamma \sigma_{P^{\prime \prime}} \mathcal{A}\left(-, v_{P}\right)$.

Define a finite graph $\mathcal{G}$ whose object-set is $(\mathrm{ob} \mathcal{P})+(\mathrm{ob} \mathcal{Q})$, and whose arrows are all those of $\mathcal{Q}$ together with arrows $x_{P}: P \longrightarrow P^{\prime}$ and $y_{P}: P \longrightarrow P^{\prime \prime}$ for each $P \in \mathcal{P}$. Let $\mathcal{S}$ be the category generated by $\mathcal{G}$ subject to the composition and identity relations that already obtain in $\mathcal{Q}$; clearly $\mathcal{S}$ is finite. Define $N: \mathcal{S} \longrightarrow \mathcal{A}$ to agree with $S$ on $\mathcal{Q}$, to send $P \in \mathcal{P}$ to $T P$, and to send $x_{P}$ and $y_{P}$ to $u_{P}$ and $v_{P}$ respectively. Then clearly colim $Y N$ is the coequalizer of $\alpha$ and $\beta$.

(It may not be inferred from the truth of Proposition 5.34 and Theorem 5.37 that, for a general cocomplete $\mathcal{C}$, the construction of the colimit-closure or of the finite-colimitclosure of a full subcategory $\mathcal{A}$ terminates after one step. The ordinary category $\mathbf{C a t}_{0}$ is by Proposition 3.40 the colimit-closure of the full subcategory $\{\mathbf{2}\}$, since $\mathbf{2}$ is a strong generator, as we observed in 5.3. The category $A$ with one object and one non-identity idempotent map is in fact easily seen to lie in the finite-colimit-closure of $\{\mathbf{2}\}$. Yet $A$ is not the colimit in $\mathbf{C a t}_{0}$ of any functor factorizing through $\{\mathbf{2}\}$; for it is soon verified that no map from a copower of $\mathbf{2}$ to $A$ is a coequalizer.)

\subsection{The filtered-colimit completion when $\mathcal{V}=$ Set; flat and left-exact functors}

For our final example with $\mathcal{V}=$ Set we consider the completion of a small $\mathcal{A}$ under small filtered colimits. Let us write this as $\widehat{\mathcal{A}} \subset\left[\mathcal{A}^{\text {op }}\right.$, Set $]$, and for the rest of this section keep $\overline{\mathcal{A}}$ for the completion of $\mathcal{A}$ under finite colimits.

A functor $F: \mathcal{A}^{\mathrm{op}} \longrightarrow$ Set is called flat if the functor $F \star-:[\mathcal{A}$, Set $] \longrightarrow$ Set is left exact that is, finite-limit-preserving. Every representable $\mathcal{A}(-, A)$ is flat, for $\mathcal{A}(-, A) \star$ ? $=($ ?) $A$ by Yoneda, which is the evaluation $E_{A}:[\mathcal{A}$, Set $] \longrightarrow$ Set, preserving all small limits. Moreover, since $\star$ is cocontinuous in both variables, it follows from Theorem 4.73 that a small filtered colimit of flat functors is flat.

Theorem 5.38 For $F: \mathcal{A}^{\mathrm{op}} \longrightarrow$ Set with $\mathcal{A}$ small, the following are equivalent:

(i) $(\mathrm{el} F)^{\mathrm{op}}$ is filtered.

(ii) The canonical expression (3.32) of $F$ as a colimit of representables is a filtered colimit.

(iii) F is some small filtered colimit of representables. 
(iv) $F$ lies in the completion $\widehat{\mathcal{A}}$ of $\mathcal{A}$ under small filtered colimits.

(v) $F$ is flat.

Proof. (i) implies (ii) since the colimit (3.32) is indexed by (el $F)^{\text {op }}$; that (ii) implies (iii) and (iii) implies (iv) are trivial. Since $\widehat{\mathcal{A}}$ is by Theorem 5.35 the closure of $\mathcal{A}$ in $\left[\mathcal{A}^{\mathrm{op}}\right.$, Set $]$ under filtered colimits, (iv) implies (v) by the remarks preceding the theorem. Thus we have only to show that (v) implies (i).

Let $T: \mathcal{P} \longrightarrow$ el $F$ where $\mathcal{P}$ is finite. As in 4.7, $T$ corresponds to a functor $S: \mathcal{P} \longrightarrow \mathcal{A}^{\text {op }}$ and an element $\alpha \in \lim F S$. By the flatness of $F$ we have $F \star \lim _{P} \mathcal{A}(S P,-) \cong \lim _{P}(F \star$ $\mathcal{A}(S P,-))$; and by Yoneda the latter is $\lim _{P} F S P=\lim F S$. By the formula (3.70) and the dual of (3.68), we have therefore a canonical surjection

$$
\sum_{A \in \mathcal{A}} F A \times \lim _{P} \mathcal{A}(S P, A) \longrightarrow \lim F S
$$

Let some inverse image of $\alpha$ under this surjection be given by $(x \in F A, \beta \in$ $\left.\lim _{P} \mathcal{A}(S P, A)\right)$. By (3.28), we can regard $\beta$ as an inductive cone in $\mathcal{A}$ over $S$ with vertex $A$. Since $F \beta_{P}: F A \longrightarrow F S P$ maps $x$ to $\alpha_{P}$ by construction, $\beta$ is equally an inductive cone in $(\mathrm{el} F)^{\mathrm{op}}$ over $T$ with vertex $(A, x)$. Thus $(\mathrm{el} F)^{\mathrm{op}}$ is filtered.

Proposition 5.39 A flat functor $F: \mathcal{A} \longrightarrow$ Set with $\mathcal{A}$ small preserves any finite limit that exists in $\mathcal{A}$. If $\mathcal{A}$ is finitely complete, the flat functors $F: \mathcal{A} \rightarrow$ Set are precisely the left-exact ones.

Proof. If $S: \mathcal{P} \longrightarrow \mathcal{A}$ with $\mathcal{P}$ finite has a limit in $\mathcal{A}$, the flatness of $F$ gives, as in the proof above, that $F \star \lim _{P} \mathcal{A}(-, S P) \cong \lim F S$. However the left side is now isomorphic to $F \star \mathcal{A}(-, \lim S) \cong F(\lim S)$. So a flat functor $F$ is certainly left exact if $\mathcal{A}$ is finitely complete. Conversely, if $\mathcal{A}$ is finitely complete and $F$ is left exact, el $F$ is finitely complete by Proposition 4.87 ; hence $(\mathrm{el} F)^{\text {op }}$ is finitely cocomplete and therefore filtered, so that $F$ is flat by Theorem 5.38.

Proposition 5.40 The finitely-presentable objects in the filtered-colimit-completion $\widehat{\mathcal{A}}$ of a small $\mathcal{A}$ are the retracts of the representables; so that they reduce to $\mathcal{A}$ if idempotents split in $\mathcal{A}$.

Proof. Since filtered colimits in $\widehat{\mathcal{A}}$ are formed as in $\left[\mathcal{A}^{\mathrm{op}}\right.$, Set $]$, the proof of Theorem 5.36 applies here virtually unchanged. The new point is that, for a finitely presentable $F$, the functor $\widehat{\mathcal{A}}(F,-)$ is only known to preserve filtered colimits; but now the canonical colimit of representables giving $F$ is filtered.

Proposition 5.41 If $\overline{\mathcal{A}}$ is the finite-colimit-completion of the small $\mathcal{A}$, then the inclusion $\overline{\mathcal{A}} \longrightarrow\left[\mathcal{A}^{\mathrm{op}}\right.$, Set $]$ is, to within equivalence, the filtered-colimit-completion $\widehat{\overline{\mathcal{A}}}$ of $\overline{\mathcal{A}}$. In consequence, every $F: \mathcal{A}^{\mathrm{op}} \longrightarrow$ Set is a filtered colimit of objects of $\overline{\mathcal{A}}$, and $\overline{\mathcal{A}}$ consists of the finitely-presentable objects of $\left[\mathcal{A}^{\mathrm{op}}\right.$, Set $]$. 
Proof. Let $K: \mathcal{A} \longrightarrow \overline{\mathcal{A}}$ be the inclusion, so that by Proposition 5.16 the inclusion $\overline{\mathcal{A}} \longrightarrow\left[\mathcal{A}^{\text {op }}\right.$, Set $]$ is $\widetilde{K}$. Using Lex for "left exact", we have by Theorem 5.38 and Proposition 5.39 that $\widehat{\mathcal{A}}$ is the full subcategory $\operatorname{Lex}\left[\overline{\mathcal{A}}^{\text {op }}\right.$, Set $]$ of $\left[\overline{\mathcal{A}}^{\text {op }}\right.$, Set $]$. By the dual of Theorem 5.35 we have an equivalence $\operatorname{Ran}_{K^{\mathrm{op}}}:\left[\mathcal{A}^{\mathrm{op}}, \operatorname{Set}\right] \simeq \operatorname{Lex}\left[\overline{\mathcal{A}}^{\mathrm{op}}\right.$, Set $]$, whose inverse is the restriction of $\left[K^{\mathrm{op}}, 1\right]$. Since the composite of $\left[K^{\mathrm{op}}, 1\right]$ with the Yoneda embedding $\overline{\mathcal{A}} \longrightarrow[\overline{\mathcal{A}}$, Set $]$ is $\widetilde{K}: \overline{\mathcal{A}} \longrightarrow\left[\mathcal{A}^{\text {op }}\right.$, Set $]$, this gives the first assertion. The second one comes from Theorem 5.38 and the third from Proposition 5.40.

Finally we give the converse to Proposition 4.74 as promised in 4.6:

Proposition $\mathbf{5 . 4 2}$ For a small $\mathcal{A}$ with finite-colimit-completion $\overline{\mathcal{A}}$, the following are equivalent:

(i) $\mathcal{A}$ is filtered.

(ii) $\operatorname{colim}:[\mathcal{A}$, Set $] \longrightarrow$ Set is left exact.

(iii) The inclusion $K: \mathcal{A} \longrightarrow \overline{\mathcal{A}}$ is final.

Proof. Since by (3.26) we have colim $F \cong \Delta 1 \star F$, what (ii) asserts is the flatness of $\Delta 1: \mathcal{A}^{\mathrm{op}} \longrightarrow$ Set. By Theorem 5.38, this is equivalent to the filteredness of $(\mathrm{el} \Delta 1)^{\mathrm{op}}$, which is $\mathcal{A}$; thus (ii) is equivalent to (i).

Since $\overline{\mathcal{A}}$ is finitely complete and hence filtered, what (iii) asserts by Proposition 4.71 is the non-emptiness of each $F / K$ for each $F \in \overline{\mathcal{A}}$. Any finite diagram in $\mathcal{A}$ has a colimit $F$ in $\overline{\mathcal{A}}$, and every $F$ in $\overline{\mathcal{A}}$ so arises. To say that the diagram admits an inductive cone with vertex $A \in \mathcal{A}$ is exactly to say that there is a map $F \longrightarrow K A$ for some $A$; thus (iii) is equivalent to $(\mathrm{i})$.

5.11 The image under $[K, 1]:[\mathcal{C}, \mathcal{B}] \longrightarrow[\mathcal{A}, \mathcal{B}]$, for a general dense $K$, of the left adjoint functors; examples and counter-examples

From the beginning of 5.4 until now we have dealt only with fully-faithful dense functors exploiting the notion of a density presentation, which does not seem to generalize usefully to the non-fully-faithful case. Now we drop this restriction, and consider an arbitrary dense $K: \mathcal{A} \longrightarrow \mathcal{C}$. For any $\mathcal{B}$, write $\operatorname{Ladj}[\mathcal{C}, \mathcal{B}]$ for the full subcategory of $[\mathcal{C}, \mathcal{B}]$ given by those $S: \mathcal{C} \longrightarrow \mathcal{B}$ which are left adjoints (or which have right adjoints); since left adjoints are certainly $\mathcal{A}$-cocontinuous, it follows from Theorem 5.1 that the restriction of $[K, 1]:[\mathcal{C}, \mathcal{B}] \longrightarrow[\mathcal{A}, \mathcal{B}]$ to $\operatorname{Ladj}[\mathcal{C}, \mathcal{B}]$ is fully-faithful, so that $\operatorname{Ladj}[\mathcal{C}, \mathcal{B}]$ is a $\mathcal{V}$-category if $\mathcal{A}$ is small, being equivalent to its replete image in $[\mathcal{A}, \mathcal{B}]$ under $[K, 1]$; the main goal of this section and the next is to determine this replete image.

In this section, devoted mostly to examples, we suppose for simplicity that $\mathcal{A}$ is small; if it were not, the expressions $\operatorname{Cocts}[\mathcal{C}, \mathcal{B}]$ and $\operatorname{Cocts}^{K}[\mathcal{C}, \mathcal{B}]$ would have to be modified. Then Proposition 5.11 and Theorem 5.33 give

$$
\operatorname{Ladj}[\mathcal{C}, \mathcal{B}]=\operatorname{Cocts}[\mathcal{C}, \mathcal{B}] \quad \text { if } \mathcal{C} \text { is cocomplete. }
$$


We also abbreviate full subcategory to subcategory, since we consider no others. Finally, we loosely write $[K, 1]$ and $\operatorname{Lan}_{K}$ for various restrictions, specified by the context, of the functors properly so named.

The following remarks, to the end of this paragraph, apply to any $K: \mathcal{A} \longrightarrow \mathcal{C}$, dense or not, and do not need $\mathcal{A}$ to be small. In Theorem 4.51, and in its generalizations Theorem 4.99 and Theorem 5.35, we have used the notation $[\mathcal{A}, \mathcal{B}]^{\prime}$ for the subcategory given by those $G: \mathcal{A} \longrightarrow \mathcal{B}$ for which $\operatorname{Lan}_{K} G$ exists. Since we have soon to deal with more than one functor $K$, we replace this notation by $[\mathcal{A}, \mathcal{B}]^{(K)}$. Similarly we have used, in Theorem 4.99 and elsewhere, $[\mathcal{C}, \mathcal{B}]^{\ell}$ for the subcategory given by those $S: \mathcal{C} \longrightarrow \mathcal{B}$ of the form $\operatorname{Lan}_{K} G$ for some $G$; we now change this to $[\mathcal{C}, \mathcal{B}]^{\ell K}$. Write $[\mathcal{A}, \mathcal{B}]_{*}^{(K)}$ for the subcategory of $[\mathcal{A}, \mathcal{B}]^{(K)}$ given by those $G$ for which the unit $G \longrightarrow\left(\operatorname{Lan}_{K} G\right) K$ is an isomorphism; and write $[\mathcal{C}, \mathcal{B}]_{*}^{\ell K}$ for the subcategory of $[\mathcal{C}, \mathcal{B}]^{\ell K}$ given by those $S$ for which the identity 1: $S K \longrightarrow S K$ exhibits $S$ as $\operatorname{Lan}_{K} S K$. It is immediate from Theorem 4.38 that we have an equivalence (with the evident unit and counit)

$$
\operatorname{Lan}_{K} \dashv[K, 1]:[\mathcal{C}, \mathcal{B}]_{*}^{\ell K} \simeq[\mathcal{A}, \mathcal{B}]_{*}^{(K)},
$$

where (as we warned) we are using $[K, 1]$ and $\operatorname{Lan}_{K}$ loosely for their restrictions. By Proposition 4.23,

$$
[\mathcal{A}, \mathcal{B}]_{*}^{(K)}=[\mathcal{A}, \mathcal{B}]^{(K)} \quad \text { and } \quad[\mathcal{C}, \mathcal{B}]_{*}^{\ell K}=[\mathcal{C}, \mathcal{B}]^{\ell K} \quad \text { for fully-faithful } K
$$

and in this special case, (5.44) becomes the equivalence

$$
\operatorname{Lan}_{K} \dashv[K, 1]:[\mathcal{C}, \mathcal{B}]^{\ell K} \simeq[\mathcal{A}, \mathcal{B}]^{(K)} \quad \text { for fully-faithful } K
$$

of Theorem 4.99: but by the same Proposition 4.23, the first equality in (5.45) fails for a general $\mathcal{B}$ (and in particular for $\mathcal{B}=\mathcal{V}$ ) whenever $K$ is not fully faithful, so that (5.46) then fails too.

Henceforth we suppose once again that $K$ is dense. As in the proof of Theorem 5.1, a cocontinuous $S: \mathcal{C} \longrightarrow \mathcal{B}$ preserves the colimit $\widetilde{K} C * K$; so that by Theorem 5.1 (iv) we have $S \in[\mathcal{C}, \mathcal{B}]_{*}^{\ell K}$ (from which, using (5.44), we re-find the conclusion of Theorem 5.1(i), that the restriction of $[K, 1]$ to $\operatorname{Cocts}[\mathcal{C}, \mathcal{B}]$ is fully faithful). We therefore have inclusions

$$
\operatorname{Ladj}[\mathcal{C}, \mathcal{B}] \subset \operatorname{Cocts}[\mathcal{C}, \mathcal{B}] \subset[\mathcal{C}, \mathcal{B}]_{*}^{\ell K} \subset[\mathcal{C}, \mathcal{B}]^{\ell K} \subset[\mathcal{C}, \mathcal{B}] .
$$

The first of these inclusions is by (5.43) an equality when $\mathcal{C}$ is cocomplete, but is proper in general - the inclusion into Set of the small full subcategory of finite sets is continuous but has no left adjoint. The third inclusion is by (5.45) an equality when $K$ is fully faithful, and in fact Theorem 5.29 then gives the common value explicitly as

$$
[\mathcal{C}, \mathcal{B}]_{*}^{\ell K}=[\mathcal{C}, \mathcal{B}]^{\ell K}=\operatorname{Cocts}^{K}[\mathcal{C}, \mathcal{B}] \quad \text { for fully-faithful } K \text {. }
$$

However the second and fourth inclusions are in general proper even when $\mathcal{V}=\operatorname{Set}, \mathcal{C}$ and $\mathcal{B}$ are complete and cocomplete, and $K$ is fully faithful. 
For an example of this, let $K: 1 \longrightarrow \mathbf{2}$ correspond to $1 \in \mathbf{2}=(0 \longrightarrow 1)$; it is clearly dense. Then $[\mathbf{2}, \mathcal{B}]$ consists of all maps $A \longrightarrow B$ in $\mathcal{B}$; while $[\mathbf{2}, \mathcal{B}]^{\ell K}=[\mathbf{2}, \mathcal{B}]_{*}^{\ell K}$ consists of those with $A=0$, the initial object of $\mathcal{B}$; and $\operatorname{Cocts}[\mathbf{2}, \mathcal{B}]=\operatorname{Ladj}[\mathbf{2}, \mathcal{B}]$ consists of those $0 \longrightarrow B$ which are epimorphisms in $\mathcal{B}$. Here of course $\operatorname{Lan}_{K}$ is fully faithful on $[\mathcal{A}, \mathcal{B}]_{*}^{(K)}=[\mathcal{A}, \mathcal{B}]^{(K)}=[\mathcal{A}, \mathcal{B}]=\mathcal{B}$; but $[K, 1]$, although necessarily fully faithful on $[\mathbf{2}, \mathcal{B}]^{\ell K}$, is not so on $[\mathbf{2}, \mathcal{B}]$ : it sends $A \longrightarrow B$ to $B \in \mathcal{B}$. The replete image under $[K, 1]$ of $\operatorname{Ladj}[\mathbf{2}, \mathcal{B}]$ is the proper subcategory of $\mathcal{B}$ given by those $B$ for which $0 \longrightarrow B$ is epimorphic.

When $K$ is not fully faithful, the second, third and fourth inclusions in (5.47) may all be proper, even when $\mathcal{V}=$ Set, $\mathcal{C}$ and $\mathcal{B}$ are complete and cocomplete, and $K$ is surjective on objects. For an example, let $K: 2 \longrightarrow \mathbf{2}$ be the inclusion, where 2 is the discrete category $\{0,1\}$, and let $\mathcal{B}=\mathbf{S e t}^{\mathrm{op}}$; again $K$ is clearly dense. An object of $[\mathbf{2}, \mathcal{B}]$ may be identified with a map $B \longrightarrow A$ in Set; this is sent by $[K, 1]$ to $(B, A) \in[2$, Set $]=$ Set $\times$ Set; and $\operatorname{Lan}_{K}$ sends $(D, C)$ to the projection $D \times C \longrightarrow C$. Clearly $[\mathbf{2}, \mathcal{B}]^{\ell K}$ consists of those $B \longrightarrow A$ isomorphic to such projections, while $[2, \mathcal{B}]_{*}^{\ell K}$ consists of such projections for which the other projection $D \times C \longrightarrow D$ is an isomorphism - that is, the maps $D \longrightarrow 1$ and the maps $0 \longrightarrow C$. Finally (as in the last example) $\operatorname{Ladj}[\mathbf{2}, \mathcal{B}]$ consists of the maps $1 \longrightarrow 1$ and $0 \longrightarrow 1$. Observe how badly (5.46) fails: although $[K, 1]$ is conservative on $[\mathbf{2}, \mathcal{B}]$ (as it must be for an essentially surjective $K$ ), it is not fully faithful on $[\mathbf{2}, \mathcal{B}]^{\ell K}$; and $\operatorname{Lan}_{K}$ is not fully faithful on $[2, \mathcal{B}]=[2, \mathcal{B}]^{(K)}$. Clearly $[2, \mathcal{B}]_{*}^{(K)}$ consists of the pairs of the forms $(D, 1)$ and $(0, C)$, while the replete image under $[K, 1]$ of $\operatorname{Ladj}[\mathbf{2}, \mathcal{B}]$ consists of the pairs $(1,1)$ and $(0,1)$.

For a non-fully-faithful $K$, not only does Proposition 4.23 fail, but even a left adjoint $S: \mathcal{C} \longrightarrow \mathcal{B}$ may be $\operatorname{Lan}_{K} G$ for some $G$ other than $S K$; in which case, by (5.44) and (5.47), $G$ must lie outside $[\mathcal{A}, \mathcal{B}]_{*}^{(K)}$. The example above does not illustrate this possibility, but the following one does so. With $\mathcal{V}=$ Set again, let $K=\Delta 1: \mathbf{2} \longrightarrow \mathbf{2}$. Since the unique map $\mathbf{2} \longrightarrow 1$ is obviously initial, the left Kan extension of $K$ along $\mathbf{2} \longrightarrow 1$ is $\Delta 1: 1 \longrightarrow \mathbf{2}$ by Theorem 4.67; since the latter functor is clearly dense, so is $K$ by Proposition 5.7. Taking $\mathcal{B}$ cocomplete, we see that $\operatorname{Lan}_{K}$ sends $C \longrightarrow D$ to $0 \longrightarrow D$, while $[K, 1]$ sends $A \longrightarrow B$ to $1: B \longrightarrow B$. Here $[\mathcal{C}, \mathcal{B}]_{*}^{\ell K}=[\mathcal{C}, \mathcal{B}]^{\ell K}$, even though $K$ is not fully faithful each consisting of the maps $0 \longrightarrow B$; while $[\mathcal{A}, \mathcal{B}]_{*}^{(K)}$ consists of the maps $1: D \longrightarrow D$. As in the penultimate example, $\operatorname{Ladj}[\mathcal{C}, \mathcal{B}]$ consists of those $0 \longrightarrow B$ which are epimorphic; and such an object is the image under $\operatorname{Lan}_{K}$ of any $\mathcal{C} \longrightarrow \mathcal{B}$, which need not in general for instance, if $\mathcal{B}=\operatorname{Set}^{\text {op }}-$ lie in $[\mathcal{A}, \mathcal{B}]_{*}^{(K)}$.

\subsection{Description of the image above in terms of $K$-comodels; generaliza- tion to an equivalence $\operatorname{Lan}_{Z} \dashv[Z, 1]$ between categories of comodels}

We now answer the question raised in 5.11 for a dense $K: \mathcal{A} \rightarrow \mathcal{C}$ by identifying the replete image of $[K, 1]: \operatorname{Ladj}[\mathcal{C}, \mathcal{B}] \longrightarrow[\mathcal{A}, \mathcal{B}]$ as a certain subcategory $K-\operatorname{Com}[\mathcal{A}, \mathcal{B}]^{(K)}$ of $[\mathcal{A}, \mathcal{B}]_{*}^{(K)} \subset[\mathcal{A}, \mathcal{B}]$; in this form, the result is independent of the smallness of $\mathcal{A}$.

We call a functor $G: \mathcal{A} \longrightarrow \mathcal{B}$ a $K$-comodel if $\widetilde{G}: \mathcal{B} \longrightarrow\left[\mathcal{A}^{\text {op }}, \mathcal{V}\right]$ factorizes through $\widetilde{K}: \mathcal{C} \longrightarrow\left[\mathcal{A}^{\mathrm{op}}, \mathcal{V}\right]$ as

$$
\widetilde{G} \cong \widetilde{K} T
$$


for some $T: \mathcal{B} \longrightarrow \mathcal{C}$. Since $\widetilde{K}$ is fully faithful by Theorem 5.1 , such a $T$ is unique to within isomorphism if it exists. We can write (5.49) more explicitly as a $\mathcal{V}$-natural isomorphism

$$
\mathcal{B}(G A, B) \cong \mathcal{C}(K A, T B)
$$

Since $\widetilde{K}$ trivially factorizes through itself, we have:

$$
\text { The dense } K: \mathcal{A} \longrightarrow \mathcal{C} \text { is always itself a } K \text {-comodel. }
$$

We write $K$-Com $[\mathcal{A}, \mathcal{B}]$ for the subcategory of $[\mathcal{A}, \mathcal{B}]$ given by the $K$-comodels, and $K-\operatorname{Com}[\mathcal{A}, \mathcal{B}]^{(K)}$ for the intersection of this with $[\mathcal{A}, \mathcal{B}]^{(K)}$ - that is, the category of those $K$-comodels $G$ for which $\operatorname{Lan}_{K} G$ exists. More generally, for any $Z: \mathcal{A} \longrightarrow \mathcal{D}$, we write $K-\operatorname{Com}[\mathcal{A}, \mathcal{B}]^{(Z)}$ for the intersection $K-\operatorname{Com}[\mathcal{A}, \mathcal{B}] \cap[\mathcal{A}, \mathcal{B}]^{(Z)}$.

The name " $K$-comodel" for a $G: \mathcal{A} \longrightarrow \mathcal{B}$ satisfying (5.49) is inspired by the fact (see 5.13 below) that a dense $K: \mathcal{A} \longrightarrow \mathcal{C}$, at least for small $\mathcal{A}$ and cocomplete $\mathcal{C}$, may be thought of as a very general kind of essentially-algebraic theory, a model of which in $\mathcal{P}$ is a functor $H: \mathcal{A}^{\text {op }} \longrightarrow \mathcal{P}$ such that $G=H^{\mathrm{op}}: \mathcal{A} \longrightarrow \mathcal{P}^{\text {op }}$ is a comodel in the sense above. These models constitute a full subcategory $K$-Mod $\left[\mathcal{A}^{\text {op }}, \mathcal{P}\right]$ of $\left[\mathcal{A}^{\text {op }}, \mathcal{P}\right]$, isomorphic to $\left(K-\operatorname{Com}\left[\mathcal{A}, \mathcal{P}^{\text {op }}\right]\right)^{\text {op }}$. If we talk here of comodels rather than models, it is because we have elected to emphasize left Kan extensions and colimits, rather than their duals. Particular examples of such theories are given in 6.2 and 6.3 below.

Rather than establish directly the promised equivalence $\operatorname{Ladj}[\mathcal{A}, \mathcal{B}] \simeq K-\operatorname{Com}[\mathcal{A}, \mathcal{B}]^{(K)}$, we first prove a more general result, itself of value in practice:

Theorem 5.52 Let the dense $K: \mathcal{A} \longrightarrow \mathcal{C}$ be (isomorphic to) the composite of $Z: \mathcal{A} \longrightarrow \mathcal{D}$ and a fully-faithful $J: \mathcal{D} \longrightarrow \mathcal{C}$ (so that, by Theorem 5.13, both $Z$ and $J$ are also dense). Then we have an equivalence

$$
\operatorname{Lan}_{Z} \dashv[Z, 1]: J-\operatorname{Com}[\mathcal{D}, \mathcal{B}] \simeq K-\operatorname{Com}[\mathcal{A}, \mathcal{B}]^{(Z)}
$$

whose unit and counit are the canonical maps.

Proof. $\quad$ Let $H \in J$ - $\operatorname{Com}[\mathcal{D}, \mathcal{B}]$, so that, corresponding to (5.50), we have

$$
\mathcal{B}(H D, B) \cong \mathcal{C}(J D, T B)
$$

for some $T: \mathcal{B} \longrightarrow \mathcal{C}$. Putting $D=Z A$ in this gives $\mathcal{B}(H Z A, B) \cong \mathcal{C}(J Z A, T B) \cong$ $\mathcal{C}(K A, T B)$; showing that $H Z$ is a $K$-comodel, and giving $(\widetilde{H Z})$ explicitly as $\widetilde{K} T$. Since (4.49) gives $\widetilde{Z} \cong \widetilde{K} J$, we have $(\widetilde{K}$ being fully faithful)

$$
\left[\mathcal{A}^{\mathrm{op}}, \mathcal{V}\right](\widetilde{Z} D,(\widetilde{H Z}) B) \cong\left[\mathcal{A}^{\mathrm{op}}, \mathcal{V}\right](\widetilde{K} J D, \widetilde{K} T B) \cong \mathcal{C}(J D, T B) \cong \mathcal{B}(H D, B),
$$

which by (4.20) shows that $H \cong \operatorname{Lan}_{Z}(H Z)$; moreover the unit of this Kan extension is easily calculated by Theorem 4.6 to be $1: H Z \longrightarrow H Z$. Since we have seen that $H Z$ is a $K$-comodel, it does indeed lie in $K$-Com $[\mathcal{A}, \mathcal{B}]^{(Z)}$.

Now let $G \in K-\operatorname{Com}[\mathcal{A}, \mathcal{B}]^{(Z)}$, so that $\widetilde{G} \cong \widetilde{K} T$ for some $T: \mathcal{B} \longrightarrow \mathcal{C}$. Writing $H$ for $\operatorname{Lan}_{Z} G$, using (4.20) for the first step, and recalling that $\widetilde{Z} \cong \widetilde{K} J$, we have

$$
\mathcal{B}(H D, B) \cong\left[\mathcal{A}^{\mathrm{op}}, \mathcal{V}\right](\widetilde{Z} D, \widetilde{G} B) \cong\left[\mathcal{A}^{\mathrm{op}}, \mathcal{V}\right](\widetilde{K} J D, \widetilde{K} T B) \cong \mathcal{C}(J D, T B),
$$


which is (5.54), showing that $H=\operatorname{Lan}_{Z} G$ belongs to $J$-Com $[\mathcal{D}, \mathcal{B}]$. Putting $D=Z A$ in (5.54) gives as before $\mathcal{B}(H Z A, B) \cong \mathcal{C}(K A, T B)$; the latter now being $\mathcal{B}(G A, B)$ by (5.50), Yoneda gives an isomorphism $G \cong H Z$, which is easily verified to be the unit $G \longrightarrow\left(\operatorname{Lan}_{Z} G\right) Z$ of the Kan extension.

Since the unit and counit of the equivalence (5.53) are the canonical maps, it follows that

$$
J-\operatorname{Com}[\mathcal{D}, \mathcal{B}] \subset[\mathcal{D}, \mathcal{B}]_{*}^{\ell Z} \quad \text { and } \quad K-\operatorname{Com}[\mathcal{A}, \mathcal{B}]^{(Z)} \subset[\mathcal{A}, \mathcal{B}]_{*}^{(Z)}
$$

and that the equivalence $(5.53)$ is just the restriction of the equivalence $[\mathcal{D}, \mathcal{B}]_{*}^{\ell Z} \cong[\mathcal{A}, \mathcal{B}]_{*}^{(Z)}$ of (5.44). Now consider the special case of Theorem 5.52 in which $\mathcal{D}=\mathcal{C}, J=1_{\mathcal{C}}$, and $Z=K$. A $1_{\mathcal{C}}$-comodel is by (5.50) a functor $S: \mathcal{C} \longrightarrow \mathcal{B}$ for which $\mathcal{B}(S C, B) \cong \mathcal{C}(C, T B)$ for some $T$; that is, it is a left-adjoint functor. Thus Theorem 5.52 (together with (5.43)) gives the following answer to the problem proposed at the beginning of 5.11:

Theorem 5.56 For a dense $K: \mathcal{A} \longrightarrow \mathcal{C}$ and any $\mathcal{B}$, the equivalence $\operatorname{Lan}_{K} \dashv$ $[K, 1]:[\mathcal{C}, \mathcal{B}]_{*}^{\ell K} \simeq[\mathcal{A}, \mathcal{B}]_{*}^{(K)}$ restricts to an equivalence

$$
\operatorname{Lan}_{K} \dashv[K, 1]: \operatorname{Ladj}[\mathcal{C}, \mathcal{B}] \simeq K-\operatorname{Com}[\mathcal{A}, \mathcal{B}]^{(K)} .
$$

Here, if $\mathcal{C}$ is cocomplete and $\mathcal{A}$ is small, $\operatorname{Ladj}[\mathcal{C}, \mathcal{B}]$ may be replaced by $\operatorname{Cocts}[\mathcal{C}, \mathcal{B}]$; and if every $G: \mathcal{A} \longrightarrow \mathcal{B}$ admits a left Kan extension along $K$, as when $\mathcal{A}$ is small and $\mathcal{B}$ is cocomplete, $K-\operatorname{Com}[\mathcal{A}, \mathcal{B}]^{(K)}$ may be replaced by $K-\operatorname{Com}[\mathcal{A}, \mathcal{B}]$.

Note that, when $\mathcal{A}$ is small and $K$ is the Yoneda embedding $Y: \mathcal{A} \longrightarrow\left[\mathcal{A}^{\text {op }}, \mathcal{V}\right]$, a $Y$-comodel is any functor $\mathcal{A} \longrightarrow \mathcal{B}$, since $\widetilde{Y}=1$ by (4.30). Thus (5.57) is another generalization of the equivalence (4.55), different from the generalization given by Theorem 5.31 . In the situation of Theorem 5.52, combining that theorem with Theorem 5.56 gives a diagram of multiple equivalences

$$
\begin{aligned}
\operatorname{Ladj}[\mathcal{C}, \mathcal{B}] \simeq J-\operatorname{Com}[\mathcal{D}, \mathcal{B}]^{(J)} & \simeq K-\operatorname{Com}[\mathcal{A}, \mathcal{D}]^{(K)} \\
\cap & \cap \\
J-\operatorname{Com}[\mathcal{D}, \mathcal{B}] & \simeq K-\operatorname{Com}[\mathcal{A}, \mathcal{D}]^{(Z)}
\end{aligned}
$$

in which the horizontal maps from left to right are restrictions, and those from right to left are left Kan extensions. The vertical inclusions here, although equalities when $\mathcal{A}$ is small and $\mathcal{B}$ is cocomplete, are in general proper, even if $\mathcal{C}$ is cocomplete and $\mathcal{A}$ and $\mathcal{D}$ are small. For let $J: \mathcal{D} \longrightarrow \mathcal{C}$ be fully faithful and dense, take $\mathcal{A}=\mathcal{D}$ and $Z=1$, and take $\mathcal{B}=\mathcal{D}$; then $1: \mathcal{D} \longrightarrow \mathcal{D}$ is a $J$-comodel, since $\widetilde{1}=Y$ is isomorphic by (4.22) to $\widetilde{J} J$; yet $\operatorname{Lan}_{J} 1_{\mathcal{D}}$ need not exist. To see this, let $\mathcal{C}=\mathcal{V}=$ Set, and let $J$ be the inclusion of the full subcategory $\{2\}$ determined by the single two-element set 2 ; since $\{1\}$ is dense in Set by (5.17), so is $\{1,2\}$ by Theorem 5.13, whence $\{2\}$ is dense by Proposition 5.20, as 1 is a retract of 2 . Clearly $\widetilde{J} 0$ is $\Delta 0$, the initial object of $\left[\{2\}^{\text {op }}\right.$, Set $]$; so that $\widetilde{J} 0 \star 1_{\mathcal{D}}$ would, if it existed, be the initial object of $\{2\}$. Since $\{2\}$ has no initial object, $\operatorname{Lan}_{J} 1_{\mathcal{D}}$ fails to exist. 


\subsection{A dense $K: \mathcal{A} \longrightarrow \mathcal{C}$, with $\mathcal{A}$ small and $\mathcal{C}$ cocomplete, as an essentially- algebraic theory with $\mathcal{C}$ as the category of algebras}

We now settle on the precise definition of an essentially-algebraic theory: namely, a dense $K: \mathcal{A} \longrightarrow \mathcal{C}$ with $\mathcal{A}$ small and $\mathcal{C}$ cocomplete. The models of the theory in $\mathcal{V}$ itself are usually called its algebras. We have:

Theorem 5.59 For any dense $K: \mathcal{A} \longrightarrow \mathcal{C}$ with $\mathcal{A}$ small, there is an equivalence $K-\operatorname{Mod}\left[A^{\mathrm{op}}, \mathcal{V}\right] \simeq \mathcal{C}^{\prime}$, where $\mathcal{C}^{\prime}$ is the subcategory of $\mathcal{C}$ given by those $\mathcal{C}$ such that $X \pitchfork \mathcal{C}$ exists for all $X \in \mathcal{V}$. Thus if $\mathcal{C}$ is cotensored, $K-\operatorname{Mod}\left[A^{\mathrm{op}}, \mathcal{V}\right] \simeq \mathcal{C}$. This is so in particular if $\mathcal{C}$ is cocomplete.

Proof. $\quad$ Since $\mathcal{V}^{\text {op }}$ is cocomplete, Theorem 5.56 gives $K$-Com $\left[\mathcal{A}, \mathcal{V}^{\text {op }}\right] \simeq \operatorname{Ladj}\left[\mathcal{C}, \mathcal{V}^{\text {op }}\right]$, or equivalently $K-\operatorname{Mod}\left[\mathcal{A}^{\mathrm{op}}, \mathcal{V}\right] \simeq\left(\operatorname{Ladj}\left[\mathcal{C}, \mathcal{V}^{\mathrm{op}}\right]\right)^{\mathrm{op}}$; and this last category is equivalent to $\operatorname{Radj}\left[\mathcal{C}^{\text {op }}, \mathcal{V}\right]$. But all right-adjoint functors $\mathcal{C}^{\text {op }} \longrightarrow \mathcal{V}$ lie in the subcategory $\mathcal{C} \subset\left[\mathcal{C}^{\text {op }}, \mathcal{V}\right]$ given by the representables, and constitute in fact the category $\mathcal{C}^{\prime}$ of the theorem. The last assertion follows from Proposition 5.15.

This leads to the following point of view. If $\mathcal{C}$ is a cocomplete category admitting a small dense subcategory, any choice of a dense $K: \mathcal{A} \longrightarrow \mathcal{C}$ with small $\mathcal{A}$ gives an essentiallyalgebraic theory, for which $\mathcal{C}$ is the category of algebras. As long as we consider models of the theory only in complete categories $\mathcal{P}$, the concept of a model is independent of the choice of $K$; a model of the theory in $\mathcal{P}$, also called a $\mathcal{C}$-object in $\mathcal{P}$, is by Theorem 5.56 just a continuous (and hence right-adjoint) functor $\mathcal{C}^{\mathrm{op}} \longrightarrow \mathcal{P}$. At this level, any continuous $\mathcal{P} \longrightarrow \mathcal{P}^{\prime}$ carries models to models; and any model in $\mathcal{P}$ is so obtained by applying the continuous functor $\mathcal{C}^{\mathrm{op}} \longrightarrow \mathcal{P}$ to the generic model in $\mathcal{C}^{\mathrm{op}}$ given by $1: \mathcal{C}^{\mathrm{op}} \longrightarrow \mathcal{C}^{\mathrm{op}}$. In terms of $K: \mathcal{A} \longrightarrow \mathcal{C}$, this generic model in $\mathcal{C}^{\text {op }}$ corresponds to $K^{\mathrm{op}}: \mathcal{A}^{\mathrm{op}} \longrightarrow \mathcal{C}^{\mathrm{op}}$, which is a model by $(5.51)$.

The choice of $K: \mathcal{A} \longrightarrow \mathcal{C}$ affects to some extent the notion of a model in $\mathcal{P}$ when $\mathcal{P}$ is not complete. If $K^{\prime}: \mathcal{A}^{\prime} \longrightarrow \mathcal{C}$ is another dense functor with $\mathcal{A}^{\prime}$ small, let $\mathcal{D}$ be the union of the full images in $\mathcal{C}$ of $K$ and of $K^{\prime}$; then the inclusion $J: \mathcal{D} \longrightarrow \mathcal{C}$ is dense by Theorem 5.13. Thus Theorem 5.52 gives, in an evident notation, $K-\operatorname{Mod}\left[\mathcal{A}^{\mathrm{op}}, \mathcal{P}\right]^{(Z)} \simeq$ $K^{\prime}-\operatorname{Mod}\left[\mathcal{A}^{\prime \text { op }}, \mathcal{P}\right]^{\left(Z^{\prime}\right)}$. It follows that $K-\operatorname{Mod}\left[\mathcal{A}^{\text {op }}, \mathcal{P}\right]$ and $K^{\prime}-\operatorname{Mod}\left[A^{\prime \text { op }}, \mathcal{P}\right]$ coincide when $\mathcal{P}$ is "complete enough" to admit all right Kan extensions along $Z: \mathcal{A} \longrightarrow \mathcal{D}$ and $Z^{\prime}: \mathcal{A}^{\prime} \longrightarrow \mathcal{D}$. A concrete example of such "sufficient completeness" will occur in 6.4 below.

We will end with three remarks. First, it is easily seen that, for any $\mathcal{P}$ and $\mathcal{P}^{\prime}$, a rightadjoint functor $\mathcal{P} \longrightarrow \mathcal{P}^{\prime}$ takes $K$-models to $K$-models. Secondly, the dense $I: \mathcal{I} \longrightarrow \mathcal{V}$ of (5.17) is "the theory of an object"; for, $\widetilde{I}$ being 1 , we have $I-\operatorname{Mod}\left[\mathcal{I}^{\text {op }}, \mathcal{P}\right] \cong \mathcal{P}$ for any $\mathcal{P}$. Thirdly, if $\mathcal{P}$ too is cocomplete with a small dense subcategory (and hence complete by Proposition 5.15), the notion of a $\mathcal{C}$-object in $\mathcal{P}$ coincides with that of a $\mathcal{P}$-object in $\mathcal{C}$ : for a right-adjoint $\mathcal{C}^{\text {op }} \longrightarrow \mathcal{P}$ has as its left adjoint $\mathcal{P} \longrightarrow \mathcal{C}^{\text {op }}$ what is in effect a right adjoint $\mathcal{P}^{\text {op }} \longrightarrow \mathcal{C}$. 
5.14 The image under $[K, 1]:[\mathcal{C}, \mathcal{B}] \longrightarrow[\mathcal{A}, \mathcal{B}]$, for a dense $K$, of the equivalences; characterization theorems for $\mathcal{C}$

We now determine the image, under the equivalence $\operatorname{Ladj}[\mathcal{C}, \mathcal{B}] \simeq K$-Com $[\mathcal{A}, \mathcal{B}]^{(K)}$ of (5.57), of the subcategory of equivalences $S: \mathcal{C} \simeq \mathcal{B}$. We do not suppose $\mathcal{A}$ small nor $\mathcal{C}$ cocomplete; but we do restrict ourselves to the simpler case of a fully-faithful dense $K: \mathcal{A} \longrightarrow \mathcal{C}$.

We thus get a criterion for a given category $\mathcal{B}$ to be equivalent to $\mathcal{C}$, in terms of there being a $K$-comodel $G: \mathcal{A} \longrightarrow \mathcal{B}$ satisfying our characterization of the image in question; this contains as a special case the criterion of Theorem 5.26 above for $\mathcal{B}$ to be equivalent to $\left[\mathcal{A}^{\text {op }}, \mathcal{V}\right]$. We give only a few applications below; numerous others can be found in the article [21] of Diers, to whom the results (in the case $\mathcal{V}=$ Set) are due.

If there is an equivalence $S \dashv T: \mathcal{B} \simeq \mathcal{C}$, the image of $S$ under (5.57) is the $K$-comodel $G=S K: \mathcal{A} \longrightarrow \mathcal{B}$. Since we have $\mathcal{B}(G A, B)=\mathcal{B}(S K A, B) \cong \mathcal{C}(K A, T B)$, the unique $T$ which, as in (5.49) or (5.50), exhibits $G$ as being a $K$-comodel, is precisely the equivalenceinverse of $S$. We therefore start with any $K$-comodel $G: \mathcal{A} \longrightarrow \mathcal{B}$, exhibited as such by $\widetilde{G} \cong \widetilde{K} T$ as in (5.49), and seek conditions for this $T$ to be an equivalence.

This isomorphism $\sigma: \widetilde{G} \cong \widetilde{K} T$, written as a $\mathcal{V}$-natural isomorphism $\mathcal{B}(G A, B) \cong$ $\mathcal{C}(K A, T B)$ as in (5.50), is (by Yoneda in the form (1.48)) of the form $\sigma=\mathcal{C}(\rho, 1) T$ for some $\mathcal{V}$-natural $\rho: K \longrightarrow T G$. The diagram (1.39) expressing the $\mathcal{V}$-naturality of $\rho$ may be written as

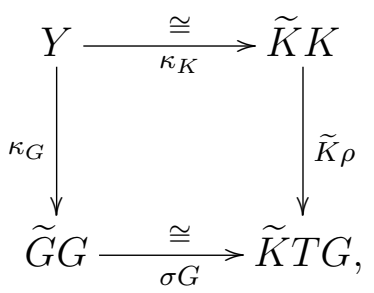

where $\kappa$ is the map of (4.22), according to which $\kappa_{G}$ is an isomorphism exactly when $G$ is fully faithful. Since $K$ is fully faithful, both the horizontal maps here are isomorphisms; whence, since $\widetilde{K}$ is fully faithful, $\rho$ is an isomorphism if and only if $G$ is fully faithful. By Theorem 5.56, $T$ admits a left adjoint $S$ precisely when $G$ admits a left Kan extension $S$ along $K$. Suppose for the moment that this is so, and let $\eta, \epsilon: S \dashv T$ be the adjunction. Then $T$ is an equivalence precisely when $\eta$ and $\epsilon$ are isomorphisms. It is clear that the isomorphism $G \cong S K$ identifies $\rho: K \longrightarrow T G$ with $\eta K: K \longrightarrow T S K$. Let $\Phi=\left(F_{\gamma}, P_{\gamma}\right)_{\gamma \in \Gamma}$ be a density presentation of $K$ as in Theorem 5.19, so that $\mathcal{C}$ is the closure of $\mathcal{A}$ under the $K$-absolute colimits $F_{\gamma} \star P_{\gamma}$ of $\Phi$. It now follows that $\eta: 1 \longrightarrow T S$ is an isomorphism if and only if $\rho$ is an isomorphism and TS preserves the colimits of $\Phi$. Because the fully-faithful $K$ both preserves and reflects these colimits, and because $\widetilde{G} \cong \widetilde{K} T$, we conclude that $T S$ preserves these colimits if and only if $\widetilde{G} S$ does so. Since the left-adjoint $S$ preserves all colimits, we have finally that $\eta$ is an isomorphism if and only if $G$ is fully faithful and the colimits $F_{\gamma} \star S P_{\gamma}$ are $G$-absolute.

Once $\eta$ is known to be an isomorphism, $T \epsilon$ is an isomorphism by the triangular equation $T \epsilon \cdot \eta T=1$ of the adjunction. So $\epsilon$ too is an isomorphism if $T$ is conservative; which of course $T$ must be if it is an equivalence. Since $\widetilde{G} \cong \widetilde{K} T$ where $\widetilde{K}$ is fully faithful, $T$ is 
conservative if and only if $\widetilde{G}$ is conservative; that is, if and only if $G: \mathcal{A} \longrightarrow \mathcal{B}$ is strongly generating. (In contrast to 3.6, we are using "strongly generating" here without thereby implying that $\mathcal{A}$ is small.)

It remains to ensure the existence of the left adjoint $S$. Propositions 3.36 and 3.37 give this if $\mathcal{B}$ admits all $F_{\gamma}$-indexed colimits for all $\gamma \in \Gamma$, but this is more than we need. The proof of Proposition 3.36 shows that we only need the (inductive) existence of the particular colimits $F_{\gamma} \star S P_{\gamma}$ in $\mathcal{B}$. Since we can run simultaneously the inductive proof of the existence of $S$ and the inductive proof that $\eta: 1 \longrightarrow T S$ is an isomorphism, we have finally:

Theorem 5.60 Let $K: \mathcal{A} \longrightarrow \mathcal{C}$ be fully faithful and dense, with density presentation $\left(F_{\gamma}, P_{\gamma}\right)_{\gamma \in \Gamma}$, and let $G: \mathcal{A} \longrightarrow \mathcal{B}$ be a $K$-comodel with $\widetilde{G} \cong \widetilde{K} T$. Then $T$ is an equivalence if and only if the following conditions are satisfied (whereupon $K \cong T G$ ):

(i) G is fully faithful.

(ii) $G$ is strongly generating (or, equivalently, $T$ is conservative).

(iii) Whenever $P_{\gamma} \cong T Q_{\gamma}$ for some $Q_{\gamma}$, the colimit $F_{\gamma} \star Q_{\gamma}$ exists in $\mathcal{B}$ and is preserved by $T$ (or, equivalently, is $G$-absolute).

This form of the theorem can be used to derive Beck's monadicity criterion; see ([21], Corollaire 5.91). The theorem takes a simpler form, sufficient for many applications, when the density presentation $\Phi$ of $K$ consists of all colimits with indexing-types in some set $\mathcal{F}$; as was the case, for instance, in Theorem 5.35 above. We then have:

Theorem 5.61 Let $\mathcal{C}$ admit all colimits with indexing types in $\mathcal{F}$, and let these colimits constitute a density presentation of the fully-faithful dense $K: \mathcal{A} \longrightarrow \mathcal{C}$. Let $G: \mathcal{A} \longrightarrow \mathcal{B}$ be a $K$-comodel with $\widetilde{G} \cong \widetilde{K} T$. Then $T$ is an equivalence if and only if the following conditions are satisfied (whereupon $K \cong T G$ ):

(i) $G$ is fully faithful and strongly generating.

(ii) $\mathcal{B}$ admits all $\mathcal{F}$-colimits and they are $\mathcal{G}$-absolute.

We can apply this to the $K: \mathcal{A} \longrightarrow \overline{\mathcal{A}}$ of Theorem 5.35 , where $\overline{\mathcal{A}}$ is the free $\mathcal{F}$ cocompletion of $\mathcal{A}$ for a set $\mathcal{F}$ of small indexing types. If $\mathcal{B}$ is to be equivalent to $\overline{\mathcal{A}}$, certainly $\mathcal{B}$ must be the closure under $\mathcal{F}$-colimits of $\mathcal{A}$, embedded in $\mathcal{B}$ by $G$. By Proposition 3.40, whose first assertion does not really need the smallness of $\mathcal{A}$, it is then automatic that $G$ is strongly generating. If, moreover, $\mathcal{F}$-colimits are $G$-absolute in $\mathcal{B}$, each $\widetilde{G} B$ in $\left[\mathcal{A}^{\text {op }}, \mathcal{V}\right]$ is an iterated $\mathcal{F}$-colimit of objects of $\mathcal{A}$, and hence lies in $\mathcal{C}$; so that $G$ is automatically a $K$-comodel. This gives:

Proposition 5.62 In order that $G: \mathcal{A} \longrightarrow \mathcal{B}$ be equivalent to the the free $\mathcal{F}$-cocompletion of $\mathcal{A}$ for a given set $\mathcal{F}$ of small indexing types, it is necessary and sufficient that $G$ be fully faithful, that $\mathcal{B}$ admit all $\mathcal{F}$-colimits and be the closure of $\mathcal{A}$ under these, and that all $\mathcal{F}$-colimits in $\mathcal{B}$ be $G$-absolute. 
Taking $\mathcal{F}$ here to consist of all small indexing types, with $\mathcal{A}$ small, does not in fact given Theorem 5.26 as stated, but a variant. Yet Theorem 5.26 does follow from Theorem 5.61; for, since $\widetilde{Y} \cong 1$, every $G: \mathcal{A} \longrightarrow \mathcal{B}$ is automatically a $Y$-comodel. 


\section{Chapter 6}

\section{Essentially-algebraic theories defined by reguli and by sketches}

\subsection{Locally-bounded categories; the local boundedness of $\mathcal{V}$ in all our examples}

All that we have so far required of the symmetric monoidal closed $\mathcal{V}$ is that $\mathcal{V}_{0}$ be locally small, complete, and cocomplete. To ensure the reflectivity of certain naturally-occurring subcategories of $\left[\mathcal{A}^{\mathrm{op}}, \mathcal{V}\right]$, we now impose upon $\mathcal{V}$ extra conditions - which are however satisfied by all the examples of symmetric monoidal closed categories given in 1.1 above.

We call upon some reflectivity results (for ordinary categories) of [44], which improve those of [30]. The reader will rightly suspect, as we proceed, that similar results could be formulated and proved directly in the context of enriched categories. The length of such an undertaking, however, would not be justified by our limited applications; we accordingly arrange, at the cost of some elegance, to use the results as they stand - appealing to Theorem 4.85 to pass from an ordinary reflexion to a $\mathcal{V}$-reflexion.

We refer to [30] for the notion of a proper factorization system $(\mathcal{E}, \mathcal{M})$ on an ordinary category $\mathcal{K}$, which for simplicity we take to be locally small, complete, and cocomplete. We recall that $\mathcal{E}$ here is a set of epimorphisms, and $\mathcal{M}$ a set of monomorphisms, each containing the isomorphisms and closed under composition, such that every map factorizes as $i p$ with $i \in \mathcal{M}$ and $p \in \mathcal{E}$, this factorization being essentially unique and functorial, in virtue of a "diagonal fill-in" property. We further recall that the $\mathcal{M}$-subobjects of an object $A \in \mathcal{K}$ form a lattice admitting small intersections and unions: the intersection $\cap B_{i}$ being the limit of the diagram $B_{i} \longrightarrow A$, while the union $\cup B_{i}$ is obtained by forming the $(\mathcal{E}, \mathcal{M})$-factorization $\sum B_{i} \longrightarrow \cup B_{i} \rightarrow A$ of the map $\sum B_{i} \rightarrow A$; and that there is a further factorization $\sum B_{i} \longrightarrow \operatorname{colim} B_{i} \longrightarrow \cup B_{i}$, both factors here being in $\mathcal{E}$. Similarly we speak of the cointersection $A \longrightarrow C$ of a set $A \longrightarrow C_{i}$ of maps in $\mathcal{E}$ : it being the colimit of the diagram, and itself in $\mathcal{E}$. Finally we recall that a small set $\mathcal{G}$ of objects of $\mathcal{K}$ is an $(\mathcal{E}, \mathcal{M})$-generator if the canonical map $\sum_{G \in \mathcal{G}} \mathcal{K}(G, A) \cdot G \longrightarrow A$ is in $\mathcal{E}$ for each $A \in \mathcal{K}$; which is equally to say that, for every proper $\mathcal{M}$-subobject $B$ of $A$, there is some $G \in \mathcal{G}$ for which some $g: G \longrightarrow A$ fails to factorize through $B$. The existence of such a generator implies that the lattice of $\mathcal{M}$-subobjects of each $A \in \mathcal{K}$ is small; that is, that 
$\mathcal{K}$ is $\mathcal{M}$-wellpowered.

We shall call a locally small, complete, and cocomplete ordinary category $\mathcal{K}$ locally bounded if there is a proper factorization system $(\mathcal{E}, \mathcal{M})$ on $\mathcal{K}$ such that

(i) every family $A \longrightarrow C_{i}$ of maps in $\mathcal{E}$, however large, admits a cointersection;

(ii) $\mathcal{K}$ has an $(\mathcal{E}, \mathcal{M})$-generator $\mathcal{G}$;

(iii) there is a small infinite regular cardinal $\alpha$ such that $\mathcal{K}(G,-): \mathcal{K} \longrightarrow$ Set preserves $\alpha$-filtered unions of $\mathcal{M}$-subobjects for each $G \in \mathcal{G}$.

Here an $\alpha$-filtered union $\cup B_{i}$ is one such that, for any subset $J$ of the indices with card $J<\alpha$, there is an index $i$ such that $B_{j} \subset B_{i}$ for each $j \in J$. To say that $\mathcal{K}(G,-)$ preserves the union is to say that $\mathcal{K}\left(G, \cup B_{i}\right)$ is the set-theoretic union of the $\mathcal{K}\left(G, B_{i}\right)$; and hence to say that any map $G \longrightarrow \cup B_{i}$ factorizes through one of the $B_{i}$.

If $A$ is any object in a locally bounded $\mathcal{K}$, there is a regular cardinal $\beta=\beta(A)$ such that $\mathcal{K}(A,-)$ preserves $\beta$-filtered unions (of $\mathcal{M}$-subobjects). We have only to choose $\beta$ at least equal to $\alpha$ and greater than $\sum_{G \in \mathcal{G}} \operatorname{card} \mathcal{K}(G, A)$. Then, if $f: A \longrightarrow \cup B_{i}$ is a map into a $\beta$-filtered union, there is for each $G \in \mathcal{G}$ and each $g: G \longrightarrow A$ some $B_{i}$ through which $f g$ factorizes, and $i$ can be chosen to be independent of $G$ and $g$; so that $f$ factorizes through $B_{i}$ by the diagonal fill-in property applied to the diagram

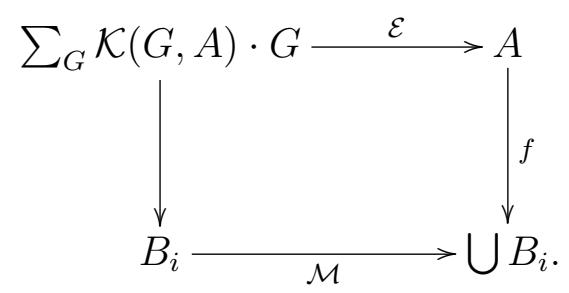

We now say that the symmetric monoidal closed $\mathcal{V}$ is locally bounded if the ordinary category $\mathcal{V}_{0}$ is locally bounded and if, moreover,

$$
(Z \otimes p) \in \mathcal{E} \text { whenever } Z \in \mathcal{V} \text { and } p \in \mathcal{E}
$$

This is clearly equivalent to

$$
[Z, i] \in \mathcal{M} \text { whenever } Z \in \mathcal{V} \text { and } i \in \mathcal{M}
$$

and combined with the diagonal fill-in property for $\mathcal{V}_{0}$, it easily implies that the diagram

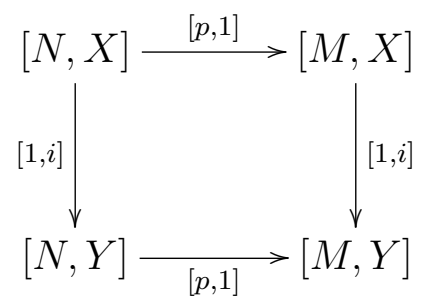


is a pullback whenever $p: M \longrightarrow N$ is in $\mathcal{E}$ and $i: X \longrightarrow Y$ is in $\mathcal{M}$. This "internal" version of the diagonal fill-in property is stronger than the original one, which we regain by applying the limit-preserving $V: \mathcal{V}_{0} \longrightarrow$ Set to the pullback (6.3).

We now verify that all the examples of symmetric monoidal closed categories in 1.1 are locally bounded. For the small preorders such as $\mathbf{2}$ and $\overline{\mathbb{R}}_{+}$, all the conditions are trivially satisfied with $\mathcal{E}=$ the epimorphisms, $\mathcal{M}=$ the extremal monomorphisms $=$ the isomorphisms.

In the examples Set, Cat, Gpd, Ord, Shv $S, \mathbf{A b}, R$-Mod, G- $R$-Mod and DG- $R$-Mod, the category $\mathcal{V}_{0}$ is locally presentable in the sense of [31]; and any such category $\mathcal{K}$ is locally bounded with $\mathcal{E}=$ the extremal epimorphisms, $\mathcal{M}=$ the monomorphisms. For it is certainly $\mathcal{E}$-cowellpowered, by Satz 7.14 of [31]; it has by definition a strong generator $\mathcal{G}$, and this is an $(\mathcal{E}, \mathcal{M})$-generator by the remarks at the end of 3.4 above; and there is by definition a regular $\alpha$ such that each $\mathcal{K}(G,-): \mathcal{K} \longrightarrow$ Set for $G \in \mathcal{G}$ preserves $\alpha$-filtered colimits - that is, each $G \in \mathcal{G}$ is $\alpha$-presentable. Now, in the presence of such a generator $\mathcal{G}$, when $\cup B_{i}$ is an $\alpha$-filtered union of subobjects, the map colim $B_{i} \longrightarrow \cup B_{i}$ is a monomorphism, as pointed out in Lemma 3.2.1 of [30]; and hence in the present case is an isomorphism, for (lying in $\mathcal{E}$ ) it is an extremal epimorphism. Thus in fact each $\mathcal{K}(G,-)$ preserves $\alpha$-filtered unions. Finally, (6.2) is satisfied in each of these examples, since the right-adjoint functor $[Z,-]$ preserves monomorphisms. (In all of these examples except $\operatorname{Shv} S$, the category $\mathcal{V}_{0}$ is locally finitely presentable, in the sense that, for a suitable choice of $\mathcal{G}$, we may take $\alpha=\aleph_{0}$. Another account of locally-presentable categories can be found in [45].)

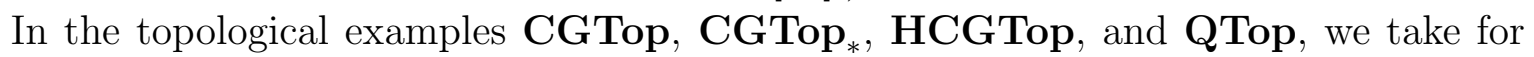
$\mathcal{M}$ the subspace-inclusions and for $\mathcal{E}$ the surjections. The first three of these are easily seen to be $\mathcal{E}$-cowellpowered. The fourth is not - the two-point set admits a large set of quasitopologies - yet it does admit all cointersections of surjections; we merely form the cointersection in Set and give it the appropriate quasitopology. The one-point space is in each case an $(\mathcal{E}, \mathcal{M})$-generator, which satisfies (iii) with $\alpha=\aleph_{0}$. Moreover, (6.1) is clearly satisfied, since the tensor product is the cartesian product - not only in $\mathcal{V}_{0}$, but also at the level of the underlying sets.

For the final example Ban, we take $\mathcal{E}=$ the epimorphisms $=$ the dense maps, $\mathcal{M}=$ the extremal monomorphisms $=$ the inclusions of closed subspaces with the induced norm. Cowellpoweredness is easy: if $X \longrightarrow Y$ is dense, $Y$ can have no more points than there are filters on the image of $X$. An $(\mathcal{E}, \mathcal{M})$-generator is $\{G\}$, where $G$ is the base-field $\mathbb{R}$ or $\mathbb{C}$. It satisfies (iii) with $\alpha=\aleph_{1}$; for then an $\alpha$-filtered union $\cup B_{i}$ is the set-theoretical union. (In general $\cup B_{i}$ is the closure of the set-theoretical union, so that every point of it is the limit of some sequence of the latter; when the union is $\aleph_{1}$-filtered, the sequence lies entirely in some $B_{i}$.) Finally (6.2) is clearly satisfied, $[Z, Y]$ being the usual function-space.

\subsection{The reflectivity in $\left[\mathcal{A}^{\mathrm{op}}, \mathcal{V}\right]$ of the category of algebras for a regulus}

The first step in making available the results of [44] is the proof of:

Proposition 6.4 If $\mathcal{V}$ is locally bounded, so is the ordinary category $\left[\mathcal{A}^{\mathrm{op}}, \mathcal{V}\right]_{0}$ for any 
$\operatorname{small} \mathcal{A}$.

ProOF. Let $(\mathcal{E}, \mathcal{M})$ be the proper factorization system on $\mathcal{V}_{0}$, and $\mathcal{G}$ the corresponding $(\mathcal{E}, \mathcal{M})$-generator. We define sets of maps in $\left[\mathcal{A}^{\text {op }}, \mathcal{V}\right]_{0}$, again called $\mathcal{E}$ and $\mathcal{M}$, by decreeing that a $\mathcal{V}$-natural $\rho: T \longrightarrow S$ is to be in $\mathcal{E}$ or in $\mathcal{M}$ precisely when all of its components $\rho_{A}: T A \longrightarrow S A$ are in the $\mathcal{E}$ or the $\mathcal{M}$ of $\mathcal{V}_{0}$. Clearly the new $\mathcal{E}$ and $\mathcal{M}$ contain the isomorphisms and are closed under composition. Since we test for a monomorphism as in 3.3 by pulling it back along itself, and since small limits in $\left[\mathcal{A}^{\mathrm{op}}, \mathcal{V}\right]$ are formed pointwise, the $\mathcal{M}$ of $\left[\mathcal{A}^{\mathrm{op}}, \mathcal{V}\right]_{0}$ consists of monomorphisms; similarly the $\mathcal{E}$ consists of epimorphisms.

To see that every $\rho: T \longrightarrow S$ factorizes into an $\mathcal{E}$ and an $\mathcal{M}$, we factorize each component $\rho_{A}$ as

$$
T A \underset{\sigma_{A}}{\longrightarrow} R A \underset{\tau_{A}}{\longrightarrow} S A
$$

with $\sigma_{A} \in \mathcal{E}$ and $\tau_{A} \in \mathcal{M}$. To make $R$ into a $\mathcal{V}$-functor $\mathcal{A}^{\text {op }} \longrightarrow \mathcal{V}$, such that $\sigma$ and $\tau$ are $\mathcal{V}$-natural, we must so define $R_{A B}$ as to render commutative the top and left regions of

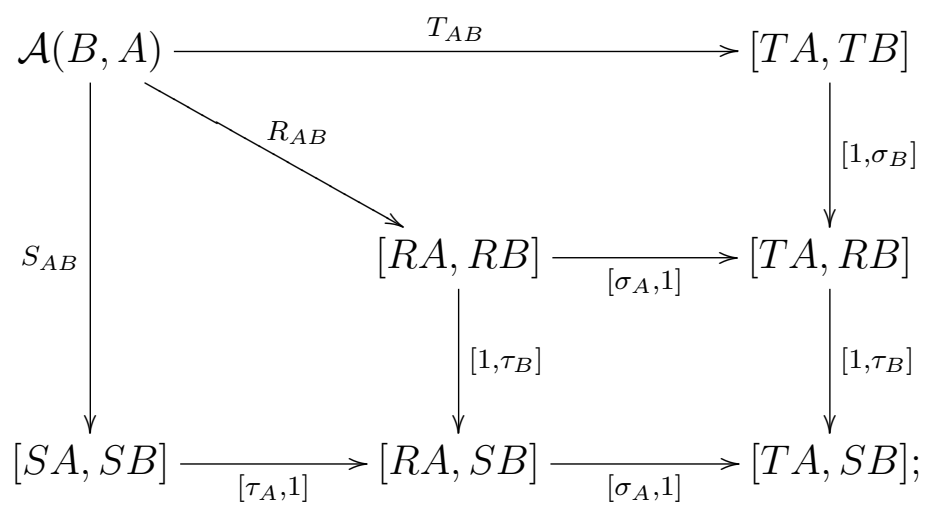

since the bottom right region is a pullback by (6.3), there is exactly one such map $R_{A B}-$ which is then easily seen to satisfy the axioms (1.5) and (1.6) for a $\mathcal{V}$-functor.

To verify the diagonal fill-in property, let $\sigma: T \longrightarrow S$ be in $\mathcal{E}$ and let $\tau: P \longrightarrow Q$ be in $\mathcal{M}$. By (6.3) we have for each $A \in \mathcal{A}$ a pullback

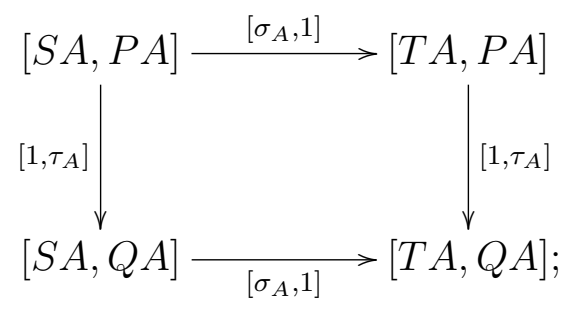

applying $\int_{A}$ to this in accordance with (2.10), and recalling from (3.20) that limits commute with limits, we get a corresponding pullback in which the top left corner is now $\left[\mathcal{A}^{\mathrm{op}}, \mathcal{V}\right](S, P)$; applying $V: \mathcal{V}_{0} \longrightarrow$ Set to this, we get a pullback in Set expressing the desired diagonal fill-in property. Hence $(\mathcal{E}, \mathcal{M})$ is a proper factorization system on $\left[\mathcal{A}^{\text {op }}, \mathcal{V}\right]_{0}$.

Write $\mathcal{H}$ for the small set $\{G \otimes \mathcal{A}(-, A)\}_{G \in \mathcal{G}, A \in \mathcal{A}}$ of objects of $\left[\mathcal{A}^{\text {op }}, \mathcal{V}\right]_{0}$. If $R$ is a proper $\mathcal{M}$-subobject of $S$, there is some $A$ for which $R A$ is a proper $\mathcal{M}$-subobject of $S A$ : and then there is some $G$ for which some $g: G \longrightarrow S A$ factorizes through $R A$. This gives 
a map $G \otimes \mathcal{A}(-, A) \longrightarrow S$ that factorizes through $R$; whence $\mathcal{H}$ is an $(\mathcal{E}, \mathcal{M})$-generator for $\left[\mathcal{A}^{\mathrm{op}}, \mathcal{V}\right]_{0}$.

Unions of $\mathcal{M}$-subobjects in $\left[\mathcal{A}^{\mathrm{op}}, \mathcal{V}\right]_{0}$ are clearly formed pointwise. Since $\left[\mathcal{A}^{\mathrm{op}}, \mathcal{V}\right]_{0}(G \otimes$ $\mathcal{A}(-, A), S) \cong \mathcal{V}_{0}(G, S A)$ by Yoneda, it follows that each $\left[\mathcal{A}^{\text {op }}, \mathcal{V}\right]_{0}(G \otimes \mathcal{A}(-, A),-)$ preserves $\alpha$-filtered unions if each $\mathcal{V}_{0}(G,-)$ does so.

An object $B$ of a $\mathcal{V}$-category $\mathcal{B}$ is said to be orthogonal in $\mathcal{B}$ to a map $\theta: M \longrightarrow N$ of $\mathcal{B}_{0}$ if the map $\mathcal{B}(\theta, 1): \mathcal{B}(N, B) \longrightarrow \mathcal{B}(M, B)$ is an isomorphism in $\mathcal{V}_{0}$. When this is so, applying $V: \mathcal{V}_{0} \longrightarrow$ Set to $\mathcal{B}(\theta, 1)$ gives an isomorphism $\mathcal{B}_{0}(\theta, 1): \mathcal{B}_{0}(N, B) \rightarrow \mathcal{B}_{0}(M, B)$ in Set, showing that $B$ is a fortiori orthogonal to $\theta$ in the ordinary category $\mathcal{B}_{0}$. In general, of course, the latter condition is strictly weaker. To ask that $\mathcal{B}(\theta, 1)$ be an isomorphism is to ask that $\mathcal{V}_{0}(X, \mathcal{B}(\theta, 1)$ ) be an isomorphism for each $X \in \mathcal{V}$ (or equivalently for each $X$ in a strong generator of $\mathcal{V}_{0}$, if it has one). If $\mathcal{B}$ is tensored, this is to ask that $B$ be orthogonal in $\mathcal{B}_{0}$ to $X \otimes \theta: X \otimes M \longrightarrow X \otimes N$ for each $X \in \mathcal{V}$.

Orthogonality sets up a Galois connexion between sets of maps and sets of objects; in the case of ordinary categories, simple closure properties of the sets so arising are listed in $\S 2$ of [30]; such of these properties as we use in the proof below are easy to verify directly.

The reflectivity result for ordinary categories that we call upon is the following case of Theorem 10.2 of [44]: Let the locally-small, complete, and cocomplete $\mathcal{K}$ be locally bounded, and let $\Theta$ be a (possibly large) set of maps $\theta: M_{\theta} \longrightarrow N_{\theta}$ in $\mathcal{K}$, such that those $N_{\theta}$ for which $\theta$ is not in $\mathcal{E}$ form (to within isomorphism) only a small set. Then the objects orthogonal in $\mathcal{K}$ to each $\theta \in \Theta$ determine a reflective full subcategory of $\mathcal{K}$. (In getting this precise form from the theorem as stated, one must recall that $\mathcal{K}$ is $\mathcal{M}$-wellpowered in virtue of the existence of the $(\mathcal{E}, \mathcal{M})$-generator.)

Our goal is the following $\mathcal{V}$-based version, in the special case where the ambient category is $\left[\mathcal{A}^{\text {op }}, \mathcal{V}\right]$; its statement makes no explicit reference to $(\mathcal{E}, \mathcal{M})$ :

Theorem 6.5 Let $\mathcal{A}$ be a small $\mathcal{V}$-category, where $\mathcal{V}$ is locally bounded. Let $\Theta$ be a set of maps $\theta: M_{\theta} \longrightarrow N_{\theta}$ in $\left[\mathcal{A}^{\mathrm{op}}, \mathcal{V}\right]_{0}$, such that the $N_{\theta}$ form (to within isomorphism) only a small set. Let $\mathcal{C}$ be the full subcategory of $\left[\mathcal{A}^{\mathrm{op}}, \mathcal{V}\right]$ determined by those objects orthogonal in $\left[\mathcal{A}^{\mathrm{op}}, \mathcal{V}\right]$ to each $\theta \in \Theta$. Then $\mathcal{C}$ is reflective in $\left[\mathcal{A}^{\mathrm{op}}, \mathcal{V}\right]$.

ProOF. It is immediate that $\mathcal{C}$ is closed under cotensor products in $\left[\mathcal{A}^{\mathrm{op}}, \mathcal{V}\right]$; hence it suffices by Theorem 4.85 to prove the reflectivity of $\mathcal{C}_{0}$ in the ordinary category $\left[\mathcal{A}^{\text {op }}, \mathcal{V}\right]_{0}$, which we now denote by $\mathcal{K}$. The objects of $\mathcal{C}_{0}$ are those orthogonal in $\mathcal{K}$ to $X \otimes \theta: X \otimes$ $M_{\theta} \longrightarrow X \otimes N_{\theta}$ for each $X \in \mathcal{V}$. If $\mathcal{G}$ is the $(\mathcal{E}, \mathcal{M})$-generator in $\mathcal{V}_{0}$, write $\epsilon_{X}: R X \longrightarrow X$ for the canonical map $\sum_{G \in \mathcal{G}} \mathcal{V}_{0}(G, X) \cdot G \longrightarrow X$, which is in $\mathcal{E}$ by hypothesis. For each $\theta$ 
and each $X$, form the commutative diagram

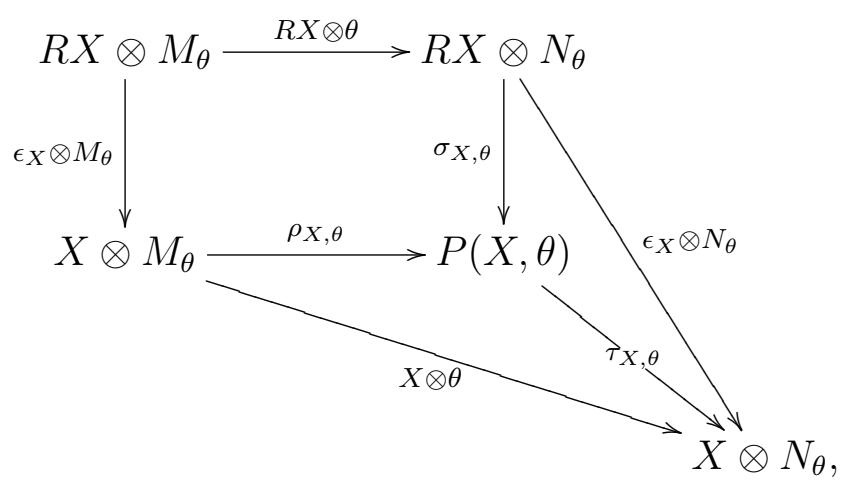

in which the top left region is a pushout. Since tensor products in $\left[\mathcal{A}^{\mathrm{op}}, \mathcal{V}\right]$ are formed pointwise, it follows from (6.1) that $\epsilon_{X} \otimes N_{\theta}$ is in $\mathcal{E}$, whence its factor $\tau_{X, \theta}$ is in $\mathcal{E}$. Every object orthogonal in $\mathcal{K}$ to $R X \otimes \theta$ is also orthogonal to its pushout $\rho_{X, \theta}$; it is then orthogonal to the composite $X \otimes \theta=\tau_{X, \theta} \rho_{X, \theta}$ if and only if it is orthogonal to $\tau_{X, \theta}$. Thus $\mathcal{C}_{0}$ consists of the objects in $\mathcal{K}$ orthogonal to each $R X \otimes \theta$ and to each $\tau_{X, \theta}$. Since $R X \otimes \theta=\left(\sum_{G} \mathcal{V}_{0}(G, X) \cdot G\right) \otimes \theta \cong \sum_{G} \mathcal{V}_{0}(G, X) \cdot(G \otimes \theta)$, any object orthogonal in $\mathcal{K}$ to each $G \otimes \theta$ is also orthogonal to the coproduct $R X \otimes \theta$ of these; and thus $\mathcal{C}_{0}$ consists of the objects in $\mathcal{K}$ orthogonal to each $G \otimes \theta$ for $G \in \mathcal{G}$ and to each $\tau_{X, \theta}$. Since the number of different $G \otimes N_{\theta}$ is small, and since each $\tau_{X, \theta}$ is in $\mathcal{E}$, the desired reflectivity of $\mathcal{C}_{0}$ in $\mathcal{K}$ follows from Proposition 6.4 and the result quoted before the theorem.

By Proposition 5.15, the cocomplete $\mathcal{V}$-category $\mathcal{C}$ of Theorem 6.5 is the category of algebras for an essentially-algebraic theory $K: \mathcal{A} \longrightarrow \mathcal{C}$, where $K$ is the composite of the Yoneda embedding $\mathcal{A} \longrightarrow\left[\mathcal{A}^{\mathrm{op}}, \mathcal{V}\right]$ and the reflexion $\left[\mathcal{A}^{\mathrm{op}}, \mathcal{V}\right] \longrightarrow \mathcal{C}$. Clearly $G: \mathcal{A} \longrightarrow \mathcal{B}$ is a comodel for the theory precisely when $\mathcal{B}(G-, B): \mathcal{A}^{\text {op }} \longrightarrow \mathcal{V}$ is for each $B \in \mathcal{B}$ orthogonal in $\left[\mathcal{A}^{\text {op }}, \mathcal{V}\right]$ to each $\theta \in \Theta$. By a very free adaptation of the terminology of Isbell [37], we shall in future call such a set $\Theta$ as occurs in Theorem 6.5 a regulus. We shall use such terms as $\Theta$-comodel and $K$-comodel interchangeably, and shall write $\Theta$-Alg for the category $\mathcal{C}$ of $K$-algebras.

\subsection{The category of algebras for a sketch, and in particular for an $\mathcal{F}$ - theory; algebraic functors and their adjoints}

We suppose henceforth that $\mathcal{V}$ is locally bounded. Let $\mathcal{A}$ be a small $\mathcal{V}$-category, and consider a set (not necessarily small)

$$
\Phi=\left\{\phi_{\gamma}: F_{\gamma} \longrightarrow \mathcal{A}\left(P_{\gamma}-, A_{\gamma}\right)\right\}_{\gamma \in \Gamma}
$$

of cylinders in $\mathcal{A}$, where $F_{\gamma}: \mathcal{L}_{\gamma}^{\text {op }} \longrightarrow \mathcal{V}$ and $P_{\gamma}: \mathcal{L}_{\gamma} \longrightarrow \mathcal{A}$, with each $\mathcal{L}_{\gamma}$ small. Call a functor $G: \mathcal{A} \longrightarrow \mathcal{B}$ a $\Phi$-comodel if, for each $\gamma$, the cylinder

$$
F_{\gamma} \underset{\phi_{\gamma}}{\longrightarrow} \mathcal{A}\left(P_{\gamma}-, A_{\gamma}\right) \underset{G}{\longrightarrow} \mathcal{B}\left(G P_{\gamma}-, G A_{\gamma}\right)
$$


is a colimit-cylinder; and write $\Phi$ - $\operatorname{Com}[\mathcal{A}, \mathcal{B}]$ for the full subcategory of $[\mathcal{A}, \mathcal{B}]$ given by these comodels. Similarly, write $\Phi-\operatorname{Mod}\left[\mathcal{A}^{\mathrm{op}}, \mathcal{P}\right]$ for $\left(\Phi-\operatorname{Com}\left[\mathcal{A}, \mathcal{P}^{\mathrm{op}}\right]\right)^{\mathrm{op}}$, and write $\Phi$-Alg for $\Phi-\operatorname{Mod}\left[\mathcal{A}^{\mathrm{op}}, \mathcal{V}\right]$. Thus the $\Phi$-algebras are those $\mathcal{A}^{\mathrm{op}} \longrightarrow \mathcal{V}$ that send each $\phi_{\gamma}$ to a limitcone. By very freely adapting the terminology of Ehresmann [24] (see also [3]), we may call the pair $\left(\mathcal{A}^{\mathrm{op}}, \Phi\right)$, or just $\Phi$ for short, a sketch.

Given such a sketch, each composite

$$
F_{\gamma} \underset{\phi_{\gamma}}{\longrightarrow} \mathcal{A}\left(P_{\gamma}-, A_{\gamma}\right) \underset{Y}{\longrightarrow}\left[\mathcal{A}^{\mathrm{op}}, \mathcal{V}\right]\left(Y P_{\gamma}-, Y A_{\gamma}\right)
$$

corresponds by (3.5) to a map

$$
\theta_{\gamma}: F_{\gamma} \star Y P_{\gamma} \longrightarrow Y A_{\gamma}
$$

in the cocomplete $\left[\mathcal{A}^{\text {op }}, \mathcal{V}\right]$. Write $\Theta=\left\{\theta_{\gamma}\right\}_{\gamma \in \Gamma}$ for the set of these maps; because $\mathcal{A}$ is small, the set of codomains in (6.10) is small, so that $\Theta$ is indeed a regulus in the sense of 6.2 .

Theorem 6.11 If the regulus $\Theta$ corresponds as above to the sketch $\Phi$, then

$$
\Phi-\operatorname{Com}[\mathcal{A}, \mathcal{B}]=\Theta-\operatorname{Com}[\mathcal{A}, \mathcal{B}]
$$

for any category $\mathcal{B}$. In particular $\Phi$-Alg is the reflective subcategory $\Theta$-Alg of $\left[\mathcal{A}^{\mathrm{op}}, \mathcal{V}\right]$. The corresponding dense functor $K: \mathcal{A} \longrightarrow \Phi$-Alg is fully faithful precisely when the cylinders $\phi_{\gamma}$ are already colimit-cylinders in $\mathcal{A}$.

Proof. By the remarks at the end of $6.2, G: \mathcal{A} \longrightarrow \mathcal{B}$ is a $\Theta$-comodel precisely when each $\mathcal{B}(G-, B)$ is orthogonal in $\left[\mathcal{A}^{\text {op }}, \mathcal{V}\right]$ to each $\theta_{\gamma}$; that is, when each

$$
\left[\mathcal{A}^{\mathrm{op}}, \mathcal{V}\right]\left(\theta_{\gamma}, 1\right):\left[\mathcal{A}^{\mathrm{op}}, \mathcal{V}\right]\left(Y A_{\gamma}, \mathcal{B}(G-, B)\right) \longrightarrow\left[\mathcal{A}^{\mathrm{op}}, \mathcal{V}\right]\left(F_{\gamma} \star Y P_{\gamma}, \mathcal{B}(G-, B)\right)
$$

is an isomorphism. By (3.5) and Yoneda, this map is isomorphic to a map

$$
\mathcal{B}\left(G A_{\gamma}, B\right) \longrightarrow\left[\mathcal{L}_{\gamma}^{\mathrm{op}}, \mathcal{V}\right]\left(F_{\gamma}, \mathcal{B}\left(G P_{\gamma}-, B\right)\right)
$$

whose unit is easily verified to be (6.8); to say that it is an isomorphism is therefore by (3.5) to say that (6.8) is a colimit-cylinder, or that $G$ is a $\Phi$-comodel. Applying this to the comodels in $\mathcal{V}^{\text {op }}$ gives by Theorem 5.59 the equality $\Phi$-Alg $=\Theta$-Alg.

By Proposition 5.16, $K$ is fully faithful if and only if $\Phi$-Alg contains the representables $\mathcal{A}(-, A)$. To ask each $\mathcal{A}(-, A)$ to be an algebra is by (6.12) to ask that each

$$
\mathcal{A}\left(A_{\gamma}, A\right) \longrightarrow\left[\mathcal{L}_{\gamma}^{\mathrm{op}}, \mathcal{V}\right]\left(F_{\gamma}, \mathcal{A}\left(P_{\gamma}-, A\right)\right)
$$

be an isomorphism, and hence by (3.5) that each $\phi_{\gamma}$ be a colimit-cylinder. 
In particular, $\Phi$-Alg is reflective in $\left[\mathcal{A}^{\mathrm{op}}, \mathcal{V}\right]$ when $\Phi$ consists of all small colimitcylinders in $\mathcal{A}$; the reflectivity of $\mathcal{V}_{0}^{\prime \prime}$ in $\mathcal{V}_{0}^{\prime}=\left[\mathcal{V}_{0}^{\text {op }}\right.$, Set' $]$ of 3.12 above is an example of this.

A very important special case of Theorem 6.11 is that where $\mathcal{A}$ is $\mathcal{F}$-cocomplete for some set $\mathcal{F}$ of small indexing-types, and where $\Phi$ consists of (the units of) all the $\mathcal{F}$ colimits in $\mathcal{A}$. In this case $\Phi$ - $\operatorname{Com}[\mathcal{A}, \mathcal{B}]=\mathcal{F}$-Cocts $[\mathcal{A}, \mathcal{B}]$ and $\Phi$-Alg $=\mathcal{F}$-Cts $\left[\mathcal{A}^{\text {op }}, \mathcal{V}\right]$. The full name $\left(\mathcal{A}^{\mathrm{op}}, \Phi\right)$ of the sketch $\Phi$ might here be replaced by $\left(\mathcal{A}^{\mathrm{op}}, \mathcal{F}\right)$. It is also suggestive to refer to a small $\mathcal{F}$-complete category $\mathcal{A}^{\text {op }}$ as an $\mathcal{F}$-theory. For instance, with $\mathcal{V}=$ Set, a Lawvere-Bénabou theory ([52], [7]) is a small category $\mathcal{A}^{\text {op }}$ with finite products; while a small $\mathcal{A}^{\mathrm{op}}$ with finite limits is sometimes called a finitary essentiallyalgebraic theory. (Joyal suggests calling the latter a cartesian theory; in that finite limits may be said to go back to Descartes, who introduced both the product $\mathbb{R} \times \mathbb{R}$ and the equalizer $\{(x, y) \mid f(x, y)=g(x, y)\}$.) This terminology moreover allows $\mathcal{A}^{\mathrm{op}}$ - $\mathrm{Alg}(\mathcal{F}$ being understood) as a useful abbreviation for $\mathcal{F}$ - $\operatorname{Cts}\left[\mathcal{A}^{\mathrm{op}}, \mathcal{V}\right]$.

Consider now a sketch $\left(\mathcal{A}^{\mathrm{op}}, \Phi\right)$ where $\Phi$ is given by (6.7); let $\mathcal{F}$ be a set of small indexing-types containing each $F_{\gamma}$ occurring in $\Phi$; and let $\mathcal{B}$ be a small $\mathcal{F}$-cocomplete category. Write $K: \mathcal{A} \longrightarrow \Phi$-Alg and $L: \mathcal{B} \longrightarrow \mathcal{B}^{\text {op }}$ - $\mathrm{Alg}=\mathcal{F}$-Cts $\left[\mathcal{B}^{\text {op }}, \mathcal{V}\right]$ for the respective dense functors, observing by Theorem 6.11 that $L$ is fully faithful. Note that, since $L$ is also an $L$-comodel by (5.51), we have:

Proposition 6.13 For a small $\mathcal{F}$-cocomplete $\mathcal{B}$, the dense inclusion $L: \mathcal{B} \rightarrow \mathcal{B}^{\mathrm{op}}$ - $\mathrm{Alg}=$ $\mathcal{F}$-Cts $\left[\mathcal{B}^{\text {op }}, \mathcal{V}\right]$ preserves and reflects $\mathcal{F}$-colimits.

Consider a $\Phi$-comodel $G: \mathcal{A} \longrightarrow \mathcal{B}$. If $Q: \mathcal{B} \longrightarrow \mathcal{D}$ is any $\mathcal{F}$-continuous functor, the composite $Q G$ is clearly another $\Phi$-comodel. Taking $\mathcal{D}=\mathcal{V}^{\text {op }}$ here, we see that $\left[G^{\text {op }}, 1\right]:\left[\mathcal{B}^{\text {op }}, \mathcal{V}\right] \longrightarrow\left[\mathcal{A}^{\text {op }}, \mathcal{V}\right]$ restricts to a functor

$$
G^{*}: \mathcal{B}^{\mathrm{op}}-\mathrm{Alg} \longrightarrow \Phi-\mathrm{Alg} .
$$

Such a functor between the categories of algebras, induced by a realization (that is, a model) $G^{\text {op }}$ of one theory in the other, is often said to be algebraic: especially in the case where $\mathcal{A}$ too is $\mathcal{F}$-cocomplete and $\Phi=\left(\mathcal{A}^{\text {op }}, \mathcal{F}\right)$.

Since $\widetilde{L}: \mathcal{B}^{\mathrm{op}}-\mathrm{Alg} \longrightarrow\left[\mathcal{B}^{\mathrm{op}}, \mathcal{V}\right]$ and $\widetilde{K}: \Phi-\mathrm{Alg} \longrightarrow\left[\mathcal{A}^{\mathrm{op}}, \mathcal{V}\right]$ are fully faithful, since

$$
\left[G^{\mathrm{op}}, 1\right]:\left[\mathcal{B}^{\mathrm{op}}, \mathcal{V}\right] \longrightarrow\left[\mathcal{A}^{\mathrm{op}}, \mathcal{V}\right]
$$

has by Theorem 4.50 the left adjoint $\operatorname{Lan}_{G^{\text {op }}}$, and since $\widetilde{L}$ has by Theorem 4.51 the left adjoint $-\star L$, it follows that the algebraic functor $\widehat{G}^{*}$ has the left adjoint

$$
G_{*}=(-\star L) \cdot \operatorname{Lan}_{G^{\mathrm{op}}} \cdot \widetilde{K}: \mathcal{B}^{\mathrm{op}}-\mathrm{Alg} \longrightarrow \Phi-\mathrm{Alg} .
$$

The value of this at $P \in \mathcal{B}^{\text {op }}-\operatorname{Alg}$ is $\left(\operatorname{Lan}_{G^{\text {op }}}(\widetilde{K} P)\right) \star L$, which by $(4.65)$ is also $(\widetilde{K} P) \star(L G)$; so that by (4.17) we have

$$
G_{*} \cong \operatorname{Lan}_{K}(L G) .
$$

In fact $G$ is recoverable from $G_{*}$. First observe that Proposition 6.13 gives: 
Proposition 6.16 In the circumstances above, $G: \mathcal{A} \longrightarrow \mathcal{B}$ is a $\Phi$-comodel if and only if $L G: \mathcal{A} \longrightarrow \mathcal{B}^{\mathrm{op}}-\mathrm{Alg}$ is a $\Phi$-comodel. Thus $\Phi$-Com $[\mathcal{A}, \mathcal{B}]$ is the full subcategory of $\Phi-\operatorname{Com}\left[\mathcal{A}, \mathcal{B}^{\mathrm{op}}-\mathrm{Alg}\right]$ given by the functors factorizing through $L$.

Using this and (6.15), we have:

Theorem 6.17 Let the small $\mathcal{B}$ be $\mathcal{F}$-cocomplete, where the set $\mathcal{F}$ of small indexingtypes contains all the $F_{\gamma}$ occurring in the sketch $\left(\mathcal{A}^{\mathrm{op}}, \Phi\right)$; and let $K: \mathcal{A} \longrightarrow \Phi$-Alg and $L: \mathcal{B} \longrightarrow \mathcal{B}^{\text {op }}$ - Alg be the respective dense functors. Then the composite of the full inclusion $\Phi-\operatorname{Com}[\mathcal{A}, \mathcal{B}] \longrightarrow \Phi-\operatorname{Com}\left[\mathcal{A}, \mathcal{B}^{\mathrm{op}}-\mathrm{Alg}\right]$ sending $G$ to $L G$, and the equivalence

$$
[K, 1] \dashv \operatorname{Lan}_{K}: \Phi-\operatorname{Com}\left[\mathcal{A}, \mathcal{B}^{\text {op }}-\mathrm{Alg}\right] \simeq \operatorname{Ladj}\left[\Phi-\mathrm{Alg}, \mathcal{B}^{\text {op }}-\mathrm{Alg}\right]
$$

of Theorem 5.56, is isomorphic to a functor

$$
()_{*}: \Phi-\operatorname{Com}[\mathcal{A}, \mathcal{B}] \longrightarrow \operatorname{Ladj}\left[\Phi-\mathrm{Alg}, \mathcal{B}^{\text {op }}-\mathrm{Alg}\right]
$$

sending $G$ to the left adjoint $G_{*}$ of $G^{*}$. Accordingly we have

$$
G_{*} K \cong L G
$$

and the functor ()$_{*}$ of (6.18) is fully faithful, its image consisting of those left-adjoint $S: \Phi-\mathrm{Alg} \longrightarrow \mathcal{B}^{\mathrm{op}}$-Alg for which $S K$ factors through the fully-faithful $L$ as $S K \cong L G$ for some (essentially unique) $G$.

We may consider this theorem in the case where $\mathcal{A}$ too is $\mathcal{F}$-cocomplete, and $\Phi=$ $\left(\mathcal{A}^{\mathrm{op}}, \mathcal{F}\right)$. The $\mathcal{F}$-theories $\mathcal{A}^{\mathrm{op}}$, the $\mathcal{F}$-continuous functors between these, and the $\mathcal{V}$ natural transformations between the latter, form a 2-category - that is, a Cat-category. In fact they form more; for $\mathcal{F}$-Cocts $[\mathcal{A}, \mathcal{B}]$ is not just a category but a $\mathcal{V}$-category. We saw in 2.3 that $\mathcal{V}$-Cat is symmetric monoidal closed; and what the $\mathcal{F}$-theories actually constitute is a $(\mathcal{V}$-Cat $)$-category. There is a $(\mathcal{V}$-Cat $)$-functor into $\mathcal{V}$-CAT sending the $\mathcal{F}$-theory $\mathcal{A}^{\text {op }}$ to $\mathcal{A}^{\mathrm{op}}$-Alg and defined on hom-objects by (6.18). It is locally fully faithful, in the sense that (6.18) is fully faithful. Theorem 6.17 above determines, in a certain sense, its local image; not as absolutely as we should like, since the criterion for $S: \mathcal{A}^{\mathrm{op}}$-Alg $\longrightarrow \mathcal{B}^{\mathrm{op}}$ - Alg to be in this local image refers back to the inclusions of $\mathcal{A}$ and $\mathcal{B}$. The remaining step in determining globally the image of this $(\mathcal{V}$-Cat)-functor is that of characterizing the $\mathcal{V}$-categories of the form $\mathcal{A}^{\mathrm{op}}$-Alg.

For this we can get something from Theorem 5.60; in the absence of any particularly simple density presentation of $K: \mathcal{A} \longrightarrow \mathcal{A}^{\mathrm{op}}-\mathrm{Alg}$, we can just use the canonical one $\widetilde{K} C \star$ $K \cong C$ from Theorem 5.19. We have:

Proposition 6.20 If the small $\mathcal{A}$ is $\mathcal{F}$-cocomplete and $K: \mathcal{A} \longrightarrow \mathcal{A}^{\mathrm{op}}$-Alg is the fullyfaithful dense functor as above, let $G: \mathcal{A} \longrightarrow \mathcal{D}$ be an $\mathcal{F}$-cocontinuous functor, and hence a $K$-comodel; and define $T: \mathcal{D} \longrightarrow \mathcal{A}^{\mathrm{op}}-\mathrm{Alg}$ by $\widetilde{G} \cong \widetilde{K} T$. In order that $T$ be an equivalence, the following conditions are necessary and sufficient:

(i) $\mathcal{D}$ is cocomplete.

(ii) $G$ is fully faithful and strongly generating. 
(iii) The canonical map $\mathcal{A}^{\mathrm{op}}-\operatorname{Alg}(K A, C) \longrightarrow \mathcal{D}\left(G A, \mathcal{A}^{\mathrm{op}}-\mathrm{Alg}(K-, C) \star G\right)$ is an isomorphism.

Proof. The conditions are clearly necessary. For their sufficiency we refer not to Theorem 5.60 but to its proof. Since $\mathcal{D}$ is cocomplete, $G$ admits a left Kan extension $S$ along $K$, which by Theorem 5.56 is a left adjoint to $T$. For $\eta$ and $\epsilon$ to be isomorphisms, we need that $G$ is fully faithful and strongly generating (which is (ii)) and that the colimit $\widetilde{K} C \star S K$ is $G$-absolute. Since $S K \cong G$, this last requirement is that $\mathcal{D}(G A, \widetilde{K} C \star G)$ be isomorphic to $\widetilde{K} C \star \mathcal{D}(G A, G-)$; and as $G$ is fully faithful the latter is $\widetilde{K} C \star \mathcal{A}(A,-)$ or $(\widetilde{K} C) A$, giving (iii).

Again this recognition theorem is not as absolute as we should like, in that condition (iii) refers in detail to $\mathcal{A}^{\mathrm{op}}$-Alg. A far better description of the image of $\mathcal{A}^{\mathrm{op}} \mapsto \mathcal{A}^{\mathrm{op}}$-Alg is often available when both $\mathcal{V}$ and $\mathcal{F}$ are suitably restricted - see for example [45]. The fact is that we are coming to the end of what can usefully be said in the present generality.

\subsection{The $\mathcal{F}$-theory generated by a small sketch}

One last series of important observations can however be made.

Call a sketch $\left(\mathcal{A}^{\mathrm{op}}, \Phi\right)$ small if the set $\Phi$ of cylinders is small. If all the $F_{\gamma}$ occurring in $\Phi$ lie in a small set $\mathcal{F}$ of indexing-types, the smallness of $\Phi$ is automatic; for the small $\mathcal{A}$ admits but a small set of cylinders with any given indexing-type.

Proposition 6.21 Let $\left(\mathcal{A}^{\mathrm{op}}, \Phi\right)$ be a small sketch which involves indexing-types only from the small set $\mathcal{F}$; and let $K: \mathcal{A} \longrightarrow \mathcal{C}=\Phi$-Alg be the corresponding dense functor. Let $\mathcal{D}$ be the closure under $\mathcal{F}$-colimits in $\mathcal{C}$ of the full image of $K$; it is small by 3.5. Let the factorization of $K$ through $\mathcal{D}$ be $Z: \mathcal{A} \longrightarrow \mathcal{D}$ followed by the full inclusion $J: \mathcal{D} \longrightarrow \mathcal{C}$; by Theorem 5.13 both $Z$ and $J$ are dense, so that in particular $\widetilde{J}: \mathcal{C} \longrightarrow\left[\mathcal{D}^{\text {op }}, \mathcal{V}\right]$ is fully faithful. We assert that the replete image of $\widetilde{J}$ is $\mathcal{F}$-Cts $\left[\mathcal{D}^{\text {op }}, \mathcal{V}\right]$.

Proof. One direction is immediate. $J$ preserves $\mathcal{F}$-colimits by the construction of $\mathcal{D}$, so that $\mathcal{C}(J-, C)$ sends them to limits in $\mathcal{V}$, and hence $\widetilde{J} C$ is $\mathcal{F}$-continuous.

Suppose now that $P^{\text {op }}: \mathcal{D}^{\text {op }} \longrightarrow \mathcal{V}$ is $\mathcal{F}$-continuous. Since $K=J Z$ is a $\Phi$-comodel by (5.51), and since the fully-faithful $J: \mathcal{D} \longrightarrow \mathcal{C}$ not only preserves but also reflects $\mathcal{F}$ colimits, $Z: \mathcal{A} \longrightarrow \mathcal{D}$ is itself a $\Phi$-comodel. Since $P$ is $\mathcal{F}$-cocontinuous, $P Z: \mathcal{A} \longrightarrow \mathcal{V}^{\text {op }}$ too is a $\Phi$-comodel. By Theorem 5.56, we have $P Z \cong S K$ where $S: \mathcal{C} \longrightarrow \mathcal{V}^{\text {op }}$ is a leftadjoint functor given by $S=\operatorname{Lan}_{K} P Z$, the unit $P Z \longrightarrow S K$ of this left Kan extension being an isomorphism. By Theorem 4.47, since $K=J Z$ with $J$ fully faithful, we have $\operatorname{Lan}_{Z} P Z \cong S J$, with unit $\lambda$ an isomorphism. Applying Theorem 4.43 to $1: P Z \longrightarrow P Z$ as in

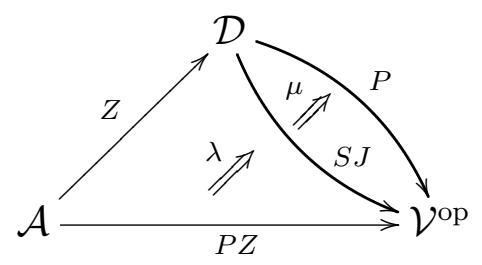


gives a map $\mu: S J \longrightarrow P$ with $\mu Z$ an isomorphism. Since both $P$ and $S J$ preserve $\mathcal{F}$ colimits, and since $\mathcal{D}$ is the closure under $\mathcal{F}$-colimits of the full image of $Z$ (which is the full image of $K$ ), we conclude that $\mu: S J \longrightarrow P$ is an isomorphism.

The right-adjoint $S^{\text {op }}: \mathcal{C}^{\text {op }} \longrightarrow \mathcal{V}$ being necessarily a representable $\mathcal{C}(-, C)$, we have $P^{\mathrm{op}} \cong S^{\mathrm{op}} J^{\mathrm{op}} \cong \mathcal{C}(J-, C)=\widetilde{J} C$, as required.

Now Theorem 6.11 gives:

Corollary 6.22 In these circumstances the functor $J$ is isomorphic to the inclusion $\mathcal{D} \longrightarrow \mathcal{D}^{\mathrm{op}}$-Alg of the $\mathcal{F}$-cocomplete small $\mathcal{D}$, so that for any $\mathcal{B}$ we have $J$-Com $[\mathcal{D}, \mathcal{B}]=$ $\mathcal{F}$-Cocts $[\mathcal{D}, \mathcal{B}]$.

Since the size of $\mathcal{F}$ here is bounded only below by the knowledge of the sketch $\Phi$, we cannot expect the comodels of the sketches $\left(\mathcal{A}^{\mathrm{op}}, \Phi\right)$ and $\left(\mathcal{D}^{\mathrm{op}}, \mathcal{F}\right)$ to "coincide" in every $\mathcal{B}$; that they do so when $\mathcal{B}$ is $\mathcal{F}$-cocomplete is an example of the "sufficient completeness" referred to in the penultimate paragraph of 5.13. The precise result is:

Theorem 6.23 In the circumstances of Proposition 6.21, every $\Phi$-comodel $G: \mathcal{A} \longrightarrow \mathcal{B}$ admits a left Kan extension along $Z: \mathcal{A} \longrightarrow \mathcal{D}$ whenever $\mathcal{B}$ is $\mathcal{F}$-cocomplete; and we have an equivalence

$$
\operatorname{Lan}_{Z} \dashv[Z, 1]: \mathcal{F}-\operatorname{Cocts}[\mathcal{D}, \mathcal{B}] \simeq \Phi-\operatorname{Com}[\mathcal{A}, \mathcal{B}]
$$

whose unit and counit are the canonical maps.

Proof. By Corollary 6.22 and Theorem 5.52, we have only to show that every $\Phi$-comodel $G: \mathcal{A} \longrightarrow \mathcal{B}$ has the form $H Z$ for some $\mathcal{F}$-continuous $H: \mathcal{D} \longrightarrow \mathcal{B}$.

We suppose first that $\mathcal{B}$ is small, and consider the inclusion $L: \mathcal{B} \longrightarrow \mathcal{B}^{\text {op }}$-Alg of Proposition 6.13. Then $L G$, being itself a $\Phi$-comodel as in Proposition 6.16, is by Theorem 5.56 isomorphic to $S K$ for some left-adjoint $S: \Phi$-Alg $\longrightarrow \mathcal{B}^{\text {op }}$-Alg; in fact $S$ is the $G_{*}$ of (6.19). The set $\mathcal{H}$ of those objects $C \in \mathcal{C}$ for which $S C$ lies in $\mathcal{B} \subset \mathcal{B}^{\text {op }}$-Alg certainly contains all the objects $K A$, since $S K \cong L G$; and it is closed under $\mathcal{F}$-colimits by Proposition 6.13 , since the left-adjoint $S$ preserves colimits; hence $\mathcal{H}$ contains $\mathcal{D}$ by the definition of this latter. Thus $S J \cong L H$ for some $H: \mathcal{D} \longrightarrow \mathcal{B}$, which is clearly $\mathcal{F}$-cocontinuous since $S J$ is so. Now $L G \cong S K=S J Z \cong L H Z$; and ( $L$ being fully faithful) we have the desired result $G \cong H Z$.

Passing to the case where the $\mathcal{F}$-cocomplete $\mathcal{B}$ need not be small, let $\mathcal{B}^{\prime}$ be the closure under $\mathcal{F}$-colimits in $\mathcal{B}$ of the full image of the $\Phi$-comodel $G: \mathcal{A} \longrightarrow \mathcal{B}$; this latter factorizing as $G^{\prime}: \mathcal{A} \longrightarrow \mathcal{B}^{\prime}$ followed by the full inclusion $N: \mathcal{B}^{\prime} \longrightarrow \mathcal{B}$. Since $N$ preserves and reflects $\mathcal{F}$-colimits, $G^{\prime}$ is itself a $\Phi$-comodel; and since $\mathcal{B}^{\prime}$ is small by 3.5 , we have as above $G^{\prime} \cong H^{\prime} Z$ for some $\mathcal{F}$-cocontinuous $H^{\prime}: \mathcal{D} \longrightarrow \mathcal{B}^{\prime}$. Now $G \cong H Z$, where $H=N H^{\prime}$ is $\mathcal{F}$-cocontinuous.

We may say that $\mathcal{D}^{\mathrm{op}}$ is the $\mathcal{F}$-theory generated by the sketch $\left(\mathcal{A}^{\mathrm{op}}, \Phi\right)$, or that $\left(\mathcal{A}^{\mathrm{op}}, \Phi\right)$ is a sketch of the $\mathcal{F}$-theory $\mathcal{D}^{\text {op }}$. The latter form of words comes closer to explicating Ehresmann's choice of the word "sketch". As he used the word (in the case $\mathcal{V}=$ Set), the small category $\mathcal{A}$ was not in general explicitly given, but presented by generators and relations; often this presentation was finite, and $\Phi$ was a finite set of finite cones (the colimits involved in $\mathcal{F}$ classically being conical ones). In such cases, one can literally see 
(the presentation of) ( $\left.\mathcal{A}^{\mathrm{op}}, \Phi\right)$ as a finitary way of "sketching" the generally infinite and complicated structure of $\mathcal{D}^{\mathrm{op}}$, or the still more complicated structure of $\mathcal{D}^{\mathrm{op}}$-Alg.

When $\mathcal{V}=$ Set and $\mathcal{F}$ consists of the functors $\Delta 1: \mathcal{L} \longrightarrow$ Set for finite $\mathcal{L}$, the $\mathcal{F}$ theories are the cartesian theories of 6.3 above; the sketch $\left(\mathcal{A}^{\mathrm{op}}, \Phi\right)$ gives rise to such a theory if $\Phi$ is a small set of finite cones. It is exactly the categories $\Phi$-Alg for such a $\Phi$ that are the locally finitely presentable categories; $\mathcal{D} \subset \Phi$-Alg is a small dense subcategory composed of objects which (since finite limits commute with filtered colimits in Set) are finitely presentable. In all the examples of categories $\mathcal{V}_{0}$ that we declared locally finitely presentable in 6.1, it is easy to see that $\mathcal{V}_{0}=\Phi$-Alg for such a sketch $\Phi$. For further information on the relation of locally presentable categories to reguli and sketches, see the classical [31], or the newer [45] which extends the ideas to certain important $\mathcal{V}$ other than Set. These $\mathcal{V}$ include Cat and Gpd, which are the appropriate base categories for the study of categories with essentially-algebraic structure. At this level, as is well known, equivalence becomes more central than isomorphism, and the naturally-occurring "functors" and "transformations" are more general than the $\mathcal{V}$-functors and the $\mathcal{V}$-natural transformations; which leads to various problems of coherence. The study of essentiallyalgebraic theories in this context must take account of these more general notions; this is not touched on in [45], but a beginning is made in [46].

\subsection{The symmetric monoidal closed structure on the category of $\mathcal{F}$ - cocomplete categories and $\mathcal{F}$-cocontinuous functors}

Before ending, we give a final application of Theorem 6.23. We consider the ( $\mathcal{V}$-Cat)category of small $\mathcal{F}$-cocomplete categories $\mathcal{A}$, where $\mathcal{F}$ is a small set of indexing-types. Since $\mathcal{F}$-Cocts $[\mathcal{A}, \mathcal{B}]$ denotes the $(\mathcal{V}$-Cat $)$-valued hom here, an appropriate name for this category is $\mathcal{F}$-Cocts.

If $\mathcal{B}$ and $\mathcal{C}$ are $\mathcal{F}$-cocomplete, so of course is $[\mathcal{B}, \mathcal{C}]$, with $\mathcal{F}$-colimits formed pointwise. The full subcategory $\mathcal{F}$-Cocts $[\mathcal{B}, \mathcal{C}]$ of this is again $\mathcal{F}$-cocomplete, since colimits commute with colimits. The functors $Q: \mathcal{A} \longrightarrow \mathcal{F}$-Cocts $[\mathcal{B}, \mathcal{C}]$ correspond to those functors $P: \mathcal{A} \otimes$ $\mathcal{B} \longrightarrow \mathcal{C}$ for which each partial functor $P(A,-): \mathcal{B} \longrightarrow \mathcal{C}$ is $\mathcal{F}$-cocontinuous. If $\mathcal{A}$ too is $\mathcal{F}$-cocomplete, the functor $Q$ is itself $\mathcal{F}$-cocontinuous precisely when the other partial functors $P(-, B): \mathcal{A} \longrightarrow \mathcal{C}$ are also $\mathcal{F}$-cocontinuous. We are thus led to the notion of a $P: \mathcal{A} \otimes \mathcal{B} \longrightarrow \mathcal{C}$ which is $\mathcal{F}$-cocontinuous in each variable separately.

Such a $P$ is a comodel for a sketch $\left((\mathcal{A} \otimes \mathcal{B})^{\text {op }}, \Phi\right)$. If $\lambda: F \rightarrow \mathcal{A}(T-, M)$ is a cylinder in $\mathcal{A}$, where $F: \mathcal{L}^{\text {op }} \longrightarrow \mathcal{V}$ and $T: \mathcal{L} \longrightarrow \mathcal{A}$, we can consider for $B \in \mathcal{B}$ the functor

$$
\mathcal{L} \cong \mathcal{L} \otimes I \underset{T \otimes B}{\longrightarrow} \mathcal{A} \otimes \mathcal{B}
$$

sending $L$ to $(T L, B)$, and called $T \otimes B$. Then we can consider the cylinder

$$
F \underset{\lambda}{\longrightarrow} \mathcal{A}(T-, M) \cong \mathcal{A}(T-, M) \otimes I \underset{1 \otimes j}{\longrightarrow} \mathcal{A}(T-, M) \otimes \mathcal{B}(B, B)=(\mathcal{A} \otimes \mathcal{B})((T-, B),(M, B)),
$$

and call this $\lambda \otimes B$. Note that this is a colimit-cylinder in $\mathcal{A} \otimes \mathcal{B}$ if $\lambda$ is one in $\mathcal{A}$; essentially because $-\otimes Y: \mathcal{V} \longrightarrow \mathcal{V}$ preserves colimits. If we take $\Phi$ to consist of all the $\lambda \otimes B$ and 
all the $A \otimes \mu$, where $\lambda$ is an $\mathcal{F}$-colimit-cylinder in $\mathcal{A}$ and $\mu$ is an $\mathcal{F}$-colimit-cylinder in $\mathcal{B}$, the $\Phi$-comodels $P: \mathcal{A} \otimes \mathcal{B} \longrightarrow \mathcal{C}$ are precisely those $P$ that are $\mathcal{F}$-cocontinuous in each variable. In fact more than this is true; it is easy to see that

$$
\Phi-\operatorname{Com}[\mathcal{A} \otimes \mathcal{B}, \mathcal{C}] \cong \mathcal{F}-\operatorname{Cocts}[\mathcal{A}, \mathcal{F} \text {-Cocts }[\mathcal{B}, \mathcal{C}]]
$$

Write $\left(\mathcal{A} \otimes_{\mathcal{F}} \mathcal{B}\right)^{\mathrm{op}}$ for the $\mathcal{F}$-theory generated by the sketch $\left((\mathcal{A} \otimes \mathcal{B})^{\mathrm{op}}, \Phi\right)$. In view of Theorem 6.23 we get

$$
\mathcal{F}-\operatorname{Cocts}\left[\mathcal{A} \otimes_{\mathcal{F}} \mathcal{B}, \mathcal{C}\right] \simeq \mathcal{F}-\operatorname{Cocts}[\mathcal{A}, \mathcal{F}-\operatorname{Cocts}[\mathcal{B}, \mathcal{C}]]
$$

The symmetry in $\mathcal{A}, \mathcal{B}, \mathcal{C}$ of $\mathcal{F}$ - $\operatorname{Cocts}[\mathcal{A}, \mathcal{F}$-Cocts $[\mathcal{B}, \mathcal{F}$-Cocts $[\mathcal{C}, \mathcal{D}]]]$ now easily gives

$$
\left(\mathcal{A} \otimes_{\mathcal{F}} \mathcal{B}\right) \otimes_{\mathcal{F}} \mathcal{C} \simeq \mathcal{A} \otimes_{\mathcal{F}}\left(\mathcal{B} \otimes_{F} \mathcal{C}\right)
$$

and

$$
\mathcal{A} \otimes_{\mathcal{F}} \mathcal{B} \simeq \mathcal{B} \otimes_{\mathcal{F}} \mathcal{A} .
$$

If $\overline{\mathcal{A}}$ is the free $\mathcal{F}$-cocompletion of $\mathcal{A}$ as in Theorem 5.35, it is clear that (for any small $\mathcal{A}$ and $\mathcal{B}$ )

$$
\overline{\mathcal{A}} \otimes_{\mathcal{F}} \overline{\mathcal{B}} \simeq \overline{\mathcal{A} \otimes \mathcal{B}}
$$

it is also clear that, for any small $\mathcal{A}$ and for $\mathcal{F}$-cocomplete $\mathcal{B}$, the $\mathcal{F}$-cocontinuous functors $\overline{\mathcal{A}} \otimes_{\mathcal{F}} \mathcal{B} \longrightarrow \mathcal{C}$ correspond to the functors $P: \mathcal{A} \otimes \mathcal{B} \longrightarrow \mathcal{C}$ for which $P(A,-)$ is $\mathcal{F}$-cocontinuous. It follows that

$$
\bar{I} \otimes_{\mathcal{F}} \mathcal{B} \simeq \mathcal{B}
$$

where $\bar{I}$ is the free $\mathcal{F}$-cocompletion of $I$; which by Theorem 5.35 is the closure of $I$ in $\mathcal{V}$ under $\mathcal{F}$-colimits.

The coherence, in the appropriate sense, of the maps (6.25), (6.26), and (6.28), follows from the universal property $(6.24)$. Thus the $(\mathcal{V}$-Cat)-category $\mathcal{F}$-Cocts is endowed with something like a symmetric monoidal closed structure - the difference being that we have equivalences in place of isomorphisms. 


\section{Bibliography}

[1] C. Auderset, Adjonctions et monades au niveau des 2-catégories, Cahiers de Top. et Géom. Diff. 15(1974), 3-20.

[2] H. Bass, Algebraic K-theory, W.A. Benjamin Inc., New York and Amsterdam, 1968.

[3] A. Bastiani and C. Ehresmann, Categories of sketched structures, Cahiers de Top. et Géom. Diff. 13(1972), 103-214.

[4] J. Bénabou, Catégories avec multiplication, C.R. Acad. Sci. Paris 256(1963), 18871890.

[5] J. Bénabou, Catégories relatives, C.R. Acad. Sci. Paris 260(1965), 3824-3827.

[6] J. Bénabou, Introduction to bicategories, Lecture Notes in Math. 47(1967), 1-77.

[7] J. Bénabou, Structures algébriques dans les catégories, Cahiers de Top. et Géom. Diff. 10 (1968), 1-126.

[8] J. Bénabou, Les distributeurs, Univ. Cath. de Louvain, Séminaires de Math. Pure, Rapport No. 33(1973).

[9] F. Borceux and G.M. Kelly, A notion of limit for enriched categories, Bull. Austral. Math. Soc. 12(1975), 49-72.

[10] M. Bunge, Relative functor categories and categories of algebras, J. Algebra 11(1969), 64-101.

[11] E. Brown, Cohomology theories, Ann. Math. 75(1962), 467-484.

[12] B.J. Day, On closed categories of functors, Lecture Notes in Math. 137(1970), 1-38.

[13] B.J. Day, Construction of biclosed categories (Ph.D. Thesis, University of New South Wales, 1970).

[14] B.J. Day, A reflection theorem for closed categories, J. Pure Appl. Algebra 2(1972), $1-11$.

[15] B.J. Day, On adjoint-functor factorization, Lecture Notes in Math. 420(1974), 1-19.

[16] B.J. Day, On closed categories of functors II, Lecture Notes in Math. 420(1974), 20-54. 
[17] B.J. Day, An embedding theorem for closed categories, Lecture Notes in Math. 420(1974), 55-64.

[18] B.J. Day, An adjoint-functor theorem over topoi, Bull. Austral. Math. Soc. 15(1976), 381-394.

[19] B.J. Day and G.M. Kelly, On topological quotient maps preserved by pullbacks or products, Proc. Cambridge Phil. Soc. 67(1970), 553-558.

[20] B.J. Day and G.M. Kelly, Enriched functor categories, Lecture Notes in Math. 106(1969), 178-191.

[21] Y. Diers, Type de densité d'une sous-catégorie pleine, Ann. Soc. Scient. Bruxelles 90(1976), 25-47.

[22] E.J. Dubuc, Kan extensions in enriched category theory, = Lecture Notes in Math. $145(1970)$.

[23] E. J. Dubuc and H. Porta, Convenient categories of topological algebras, and their duality theory, J. Pure Appl. Algebra 3(1971), 281-316.

[24] C. Ehresmann, Esquisses et types de structures algégriques, Bul. Inst. Polit. Iasi 14(1968), 1-14.

[25] S. Eilenberg and G.M. Kelly, A generalization of the functorial calculus, J. Algebra 3(1966), 366-375.

[26] S. Eilenberg and G.M. Kelly, Closed categories, Proc. Conf. on Categorical Algebra (La Jolla, 1965), Springer-Verlag, Berlin-Heidelberg-New York, 1966; 421-562.

[27] J. Fisher-Palmquist and P.H. Palmquist, Morita contexts of enriched categories, Proc. Amer. Math. Soc. 50(1975), 55-60.

[28] P.J. Freyd, Abelian categories: an introduction to the theory of functors, Harper and Row, New York, Evanston, and London, 1964. Available as TAC Reprints number 3, http://www.emis.de/journals/TAC/reprints/articles/3/tr3abs.html

[29] P.J. Freyd, Several new concepts: lucid and concordant functors, pre-limits, precompleteness, the continuous and concordant completions of categories, Lecture Notes in Math. 99(1969), 196-241.

[30] P.J. Freyd and G.M. Kelly, Categories of continuous functors I, J. Pure Appl. Algebra 2(1972), 169-191; Erratum, J. Pure Appl. Algebra 4(1974), 121.

[31] P. Gabriel and F. Ulmer, Lokal präsentierbare Kategorien, = Lecture Notes in Math. 221(1971).

[32] J.W. Gray, Closed categories, lax limits and homotopy limits, J. Pure Appl. Algebra 19(1980), 127-158. 
[33] M.F. Gouzou and R. Grunig, U-distributeurs et catégories de modules, Manuscript, Univ. de Paris, 7, 1975.

[34] A. Grothendieck, Catégories fibrées et descente, Séminaire de géometrie algébrique de l'I.H.E.S., Paris, 1961.

[35] J.R. Isbell, Adequate subcategories, Illinois J. Math. 4(1960), 541-552.

[36] J.R. Isbell, Structure of categories, Bull. Amer. Math. Soc. 72(1966), 619-655.

[37] J.R. Isbell, General functorial semantics I, Amer. J. Math. 94(1972), 535-596.

[38] D.N. Kan, Adjoint functors, Trans. Amer. Math. Soc. 87(1958), 294-329.

[39] G.M. Kelly, On Mac Lane's conditions for coherence of natural associativities, commutativities, etc., J. Algebra 1(1964), 397-402.

[40] G.M. Kelly, Tensor products in categories, J. Algebra 2(1965), 15-37.

[41] G.M. Kelly, Monomorphisms, epimorphisms, and pull-backs, J. Austral. Math. Soc. 9(1969), 124-142.

[42] G.M. Kelly, Adjunction for enriched categories, Lecture Notes in Math. 420(1969), 166-177.

[43] G.M. Kelly, Doctrinal adjunction, Lecture Notes in Math. 420(1974), 257-280.

[44] G.M. Kelly, A unified treatment of transfinite constructions for free algebras, free monoids, colimits, associated sheaves, and so on, Bull. Austral. Math. Soc. 22(1980), $1-83$.

[45] G.M. Kelly, Structures defined by finite limits in the enriched context. I. Third Colloquium on Categories, Part VI (Amiens, 1980). Cahiers Topologie Géom. 23 (1982), $3-42$.

[46] G.M. Kelly, Categories with structure - biadjoints for algebraic functors, to appear.

[47] G.M. Kelly and S. Mac Lane, Coherence in closed categories, J. Pure Appl. Algebra 1(1971), 97-140; Erratum, J. Pure Appl. Algebra 1(1971), 219.

[48] G.M. Kelly and S. Mac Lane, Closed coherence for a natural transformation, Lecture Notes in Math. 281(1972), 1-28.

[49] G.M. Kelly and R.H. Street, Review of the elements of 2-categories, Lecture Notes in Math. 420(1974), 75-103.

[50] J.F. Kennison, On limit-preserving functors, Illinois J. Math. 12(1968), 616-619.

[51] A. Kock, Closed categories generated by commutative monads, J. Austral. Math. Soc. 12(1971), 405-424. 
[52] F.W. Lawvere, Functorial semantics of algebraic theories, Proc. Nat. Acad. Sci. U.S.A. 50(1963), 869-872.

[53] F.W. Lawvere, Teoria delle categorie sopra un topos di base, Mimeographed notes, Perugia 1972/1973.

[54] F.W. Lawvere, Metric spaces, generalized logic, and closed categories, Rend. del Sem. Mat. e Fis. di Milano, 43(1973), 135-166. Available as TAC Reprints number 1, http://www.emis.de/journals/TAC/reprints/articles/1/tr1abs.html

[55] H. Lindner, Morita equivalences of enriched categories. Cahiers de Top. et Géom. Diff. 15(1974), 377-397.

[56] F.E.J. Linton, Autonomous categories and duality of functors, J. Algebra 2(1965), 315-349; Erratum, J. Algebra 4(1966), 172.

[57] F.E.J. Linton, Relative functorial semantics: adjointness results, Lecture Notes in Math. 99(1969), 384-418.

[58] S. Mac Lane, Natural associativity and commutativity, Rice Univ. Studies 49(1963), $28-46$.

[59] S. Mac Lane, Categorical algebra, Bull. Amer. Math. Soc. 71(1965), 40-106.

[60] S. Mac Lane, Categories for the Working Mathematician, Springer-Verlag, New YorkHeidelberg-Berlin, 1971.

[61] J.P. May, The geometry of iterated loop spaces, Lecture Notes in Math. 271(1972).

[62] J.P May, Pairings of categories and specta, J. Pure Appl. Algebra 19(1980), 299-346.

[63] B. Mitchell, Low dimensional group cohomology as monoidal structures, Amer. J. Math. 105 (1983), 1049-1066.

[64] Ju. A. Mitropol'skii and L.A. Nazorova, editors: Matrix Problems, Akad. Nauk Ukrain, SSR Inst. Mat., Kiev, 1977. (in Russian)

[65] K. Morita, Duality for modules and its application to the theory of rings with minimum condition, Sci. Rep. Tokoyo Kyoiku Daigaku, Sect. A, 6(1958), 83-142.

[66] D. Newell, Morita theorems for functor categories, Trans. Amer. Math. Soc. 168(1972), 423-434.

[67] J. Wick Pelletier, Dual functors and the Radon-Nikodym property in the category of Banach spaces, J. Austral. Math. Soc. (Series A) 27(1979), 479-494.

[68] S.V. Polin, Morita equivalence and the Jacobson-Rees theorems for rings and monoids in closed categories, Soviet Math. Doklady 15(1974), 1248-1251.

[69] E. Spanier, Quasi-topologies, Duke Math. J. 30(1963), 1-14. 
[70] R.H. Street, The formal theory of monads, J. Pure Appl. Algebra 2(1972), 149-168.

[71] R.H. Street, Fibrations and Yoneda's lemma in a 2-category, Lecture Notes in Math. 420(1974), 104-133.

[72] R.H. Street, Elementary cosmoi I, Lecture Notes in Math. 420(1974), 134-180.

[73] R.H. Steet, extract from a letter to F. Foltz; to appear in Diagrammes.

[74] R.H. Street and R.F.C. Walters, The comprehensive factorization of a functor, Bull. Amer. Math. Soc. 79(1973), 936-941.

[75] R.F.C. Walters, Sheaves and Cauchy-complete categories, Third Colloquium on Categories, Part IV (Amiens, 1980). Cahiers Topologie Géom. Diff. 22 (1981), 283-286.

[76] R.F.C. Walters, Sheaves on sites as Cauchy-complete categories. J. Pure Appl. Algebra 24 (1982), 95-102. 


\section{Index}

Absolute colimit, 101

$K$-absolute colimit, 92

Accessible functor, 77

weakly accessible functor, 78

Adequate, 86

adjoint

partial, 23

Adjoint functor theorem

general, 79

special, 80

Adjoint, left, 23

Adjoint, right, 23

Adjunction, 24

Algebra, 109, 119

for a regulus, 118

for a sketch, 119

Algebraic functor, 120

Atom, 80

Bicategory, 4

Biclosed

bicategory, 14

monoidal category, 14

Boolean algebra, 15

$\mathcal{C}$-object in $\mathcal{P}, 109$

$C$-object in $P, 109$

Cartesian theory, 120

Category

2-category, 8

cartesian closed, 13

cartesian monoidal, 7

closed, 13

enriched over $\mathcal{V}, 8$

filtered, 71

finite, 71

functor category, 30 monoidal, 7

monoidal biclosed, 14

monoidal closed, 13

of elements of $F, 22$

underlying, 10

Cauchy complete, 95

Cauchy completion, 95

Closed, 7

Closed monoidal category, 13

Closure

under a family of colimits, 45, 82

under a family of cylinders, 82

Cocomplete, 27

$\mathcal{F}$-cocomplete, 99

finitely, 71

Cocompletion, free, 67

Cocontinuous, 39, 99

$\mathcal{F}$-cocontinuous, 99

Codense, 86

Coend, 53

Cogenerator, strong, 46

Coherence, 124

Coherence axioms, 7, 11

Cointersection, 113

Colimit

absolute, 101

classical, 42

conical, 42, 50, 52

$\mathcal{F}$-colimits, 99

filtered, 72

indexed, 38

iterated, 51

$K$-absolute, 92

pointwise, 40

Comodel, 106

for a regulus, 118 
for a sketch, 119

Complete, 27, 50

finitely, 71

Completion

Cauchy, 95

idempotent-splitting, 100

under $\mathcal{F}$-colimits, 100

under finite colimits, 101

Component

connected, 44

Components

of a $\mathcal{V}$-natural transformation, 9

Composition law, 8

Comprehensive factorization system, 73

Cone, 42

canonical inductive, 43

projective, 42

Conical limit or colimit

classical, 42

in a $\mathcal{V}$-category, 49, 50, 52

Connected component, 44

Conservative, 8, 46

Continuity of a limit in its index, 42

Continuous, 39

Contravariant functor, 12 representable, 15

Copower, 48

Cotensor product, 48

Cotensored, 48

Counit

of a general end, 52

of a limit, 37

of a representation, 22

of a right Kan extension, 60

of an adjunction, 23

of an end in $\mathcal{V}, 27$

Cowellpoweredness, weak, 47

Cylinder, 38, 118-120, 122, 124

$K$-cylinder, 81

Dense, 86

Dense functors, 119-122

composition of, 87

Density presentation, 121
Density, formulations for, 85

Diagonal fill-in, 113

Diagram, 37

Directed, 72

Discrete Grothendieck construction, 74

Discrete op-fibration, 73

Distributor, 95

Dual $\mathcal{V}$-category, 12

$(\mathcal{E}, \mathcal{M})$-generator, 113

Element

of $X \in \mathcal{V}, 8$

of a set-valued functor, 29

End

in $\mathcal{V}, 27$

in any $\mathcal{V}$-category, 52

Epimorphism, extremal, 44

Equivalence, 24

Essentially surjective, 24

Essentially-algebraic

structure, 124

theory, 107, 109, 124

finitary, 120

Extraordinary $\mathcal{V}$-naturality, 17

Extremal epimorphism, 44

$\mathcal{F}$-cocomplete, 99

$\mathcal{F}$-Cocontinuous, 99

$\mathcal{F}$-colimits, 99

$\mathcal{F}$-theory, 120

generated by a sketch, 123, 125

Factorization system, 74

comprehensive, 73

proper, 113

Filtered category, 71

$\alpha$-filtered, 73

Filtered colimit, 72

Filtered-colimit completion, 102

Final diagram, 70

Final functor, 70

Finitary essentially-algebraic theory, 120

Finite-colimit completion, 101

Finitely cocomplete, 71

Finitely complete, 71

Finitely presentable, 83 
Flat, 102

Free cocompletion, 67

Free $\mathcal{V}$-category, 35

Fubini theorem

for ends in $\mathcal{V}, 29$

general, 41

Full image, 25

Full subcategory, 9

Fully faithful

locally, 121

Fully faithful functor, 9

Functor

2-functor, 10

algebraic, 120

conservative, 8

contravariant, 12

contravariant representable, 15

enriched over $\mathcal{V}, 9$

flat, 102

fully faithful, 9

generating, 46

of two variables, 12

partial, 12

representable, 15, 22

underlying, 10

Functor category, 30

General adjoint functor theorem, 79

Generating functor, 46

Generator, 46

$(\mathcal{E}, \mathcal{M})$-generator, 113

regular, 81

strong, 46

Generic model, 109

Grothendieck construction

discrete, 74

Heyting algebra, 15

Hom, internal, 14

Idempotent monad, 25

Idempotent, split, 100

Idempotent-splitting completion, 100

Identity element, 8

Image full, 25

replete, 25

Indexed colimit, 38

Indexed limit, 37

Indexing type, 37

Inductive cone, canonical, 43

Initial diagram, 69

Initial functor, 70

Interchange

of ends in $\mathcal{V}, 29$

of limits in general, 41

Internal hom, 14

Intersection of subobjects, 80

of $\mathcal{M}$-subobjects, 113

Iterated colimit, 51

$K$-absolute colimit, 92

$K$-comodel, 106

$K$-cylinder, 81

$K$-model, 107

Kan adjoint, 67

Kan extension, 60, 61

pointwise, 65

weak, 65

Left adequate, 86

Left adjoint, 23

Left exact, 71

Left Kan extension, 61

weak, 65

Limit

classical, 42

conical, 42, 49, 52

indexed, 37

pointwise, 40

Limit cone, 42, 49

Locally bounded, 114, 118

Locally finitely presentable, 115, 124

Locally fully faithful, 121

Locally presentable, 115

Locally small, 8, 10

$\mathcal{M}$-subobject, 113

$\mathcal{M}$-wellpowered, 114

Mates, 25 
Model, 107

for a regulus, 118

for a sketch, 119

generic, 109

Monad, idempotent, 25

Monoidal category, 7

biclosed, 14

cartesian, 7

closed, 13

strict, 8

symmetric, 12

Morita equivalence, 95

Opposite $\mathcal{V}$-category, 12

Orthogonal, 117, 119

Partial adjoint, 23

Partial functor, 12

Pointwise Kan extension, 65

Pointwise limit or colimit, 40

Pointwise limits, preservation of, 42

Power, 48

Presentation of density, 93

Preservation

of a limit, 39

of a right Kan extension, 60

Profunctors, 95

Projective cone, 42

Proper factorization system, 113

Realization, 120

Reflect limits, 44

Reflective full subcategory, 25

Regular generator, 81

Regulus, 118, 119

Replete full subcategory, 25

Replete image, 25

Repletion, 25

Representable $\mathcal{V}$-functor, 15, 22

Representation, 22

Right adequate, 86

Right adjoint, 23

Right exact, 71

Right Kan extension, 60

Sketch, 119, 123 of an $\mathcal{F}$-theory, 123

small, 122

Small, 10, 33

Small limit

$\alpha$-small, 73

Small-projective, 94

Small sketch, 122

Small $\mathcal{V}$-category, 9, 27

Solution-set condition, 79

Special Adjoint Functor Theorem, 80

Split coequalizer diagram, 81

Split idempotent, 100

Strict monoidal category, 8

Strong cogenerator, strongly cogenerating, 46

Strong generator, strongly generating, 46, 121

Subobject, 80

$\mathcal{M}$-subobject, 113

Surjective, essentially, (on objects), 24

Symmetric, 7

Symmetric monoidal category, 12

Symmetry, 11

Tensor product, 8,48

of $\mathcal{F}$-theories, 124

of $\mathcal{V}$-theories, 12

Tensored, 48

Theory, cartesian, 120

Theory, essentially-algebraic, 107, 109

$\mathcal{F}$-theory, 120

finitary, 120

Transformation, $\mathcal{V}$-natural, 9

Triangular equations, 23

Underlying category, 10

Underlying functor, 10

Union, of $\mathcal{M}$-subobjects, 113

Unit object, 8

Unit of $\mathrm{a}(\mathrm{n})$

adjunction, 23

colimit, 38

left Kan extension, 61

representation, 22

Unit $\mathcal{V}$-category, 10 
Universal $\mathcal{V}$-natural family, 27

$\mathcal{V}$-category, 8 cocomplete, 39

complete, 39

dual, 12

opposite, 12

small, 9, 27

unit, 10

$\mathcal{V}$-category

free, 35

$\mathcal{V}$-functor, 9

accessible, 77

cocontinuous, 39

conservative, 46

continuous, 39

dense, 86

representable, 15, 22

strongly cogenerating, 46

strongly generating, 46

$\mathcal{V}$-natural family

extraordinary, 17

universal, 27

$\mathcal{V}$-natural transformation, 9

Weak Kan extension, 65

Weakly accessible, 78

Weakly cowellpowered, 47

Wellpowered: $\mathcal{M}$-wellpowered, 114

Yoneda embedding, 34

Yoneda isomorphism, 33, 39

Yoneda lemma

strong, 33

weak, 21 
REPRINTS IN THEORY AND APPLICATIONS OF CATEGORIES will disseminate articles from the body of important literature in Category Theory and closely related subjects which have never been published in journal form, or which have been published in journals whose narrow circulation makes access very difficult. Publication in 'Reprints in Theory and Applications of Categories' will permit free and full dissemination of such documents over the Internet. Articles appearing have been critically reviewed by the Editorial Board of Theory and Applications of Categories. Only articles of lasting significance are considered for publication. Distribution is via the Internet tools WWW/ftp.

SUBSCRIPTION INFORMATION Individual subscribers receive (by e-mail) abstracts of articles as they are published. To subscribe, send e-mail to tac@mta.ca including a full name and postal address. For institutional subscription, send enquiries to the Managing Editor.

SELECTION OF REPRINTS After obtaining written permission from any copyright holder, any three TAC Editors may propose a work for TAC Reprints to the Managing Editor. The proposal will be reported to all Editors. The Managing Editor may either accept the proposal or require that the Editors vote on it. Before such a vote, the author, title and original publication data will be circulated to Editors. If a 2/3 majority of those TAC Editors responding within one month agrees, the work will be accepted for TAC Reprints. After a work is accepted, the author or proposer must provide to TAC either a usable TeX source or a PDF document acceptable to the Managing Editor that reproduces a typeset version. Up to five pages of corrections, commentary and forward pointers may be appended by the author. When submitting commentary, authors should read and follow the Format for submission of Theory and Applications of Categories at http://www.tac.mta.ca/tac/.

MANAGING EDITOR Robert Rosebrugh, Mount Allison University: rrosebrugh@mta.ca

TEXnICAL EDITOR Michael Barr, McGill University: barr@math.mcgill.ca

Assistant TEX EDitor Gavin Seal, Ecole Polytechnique Fédérale de Lausanne: gavin_seal@fastmail.fm

\section{TRANSMITTING EDITORS}

Clemens Berger, Université de Nice-Sophia Antipolis, cberger@math.unice.fr

Richard Blute, Université d' Ottawa: rblute@uottawa.ca

Lawrence Breen, Université de Paris 13: breen@math.univ-paris13.fr

Ronald Brown, University of North Wales: ronnie.profbrown(at)btinternet.com

Valeria de Paiva: valeria.depaiva@gmail.com

Ezra Getzler, Northwestern University: getzler (at)northwestern(dot)edu

Kathryn Hess, Ecole Polytechnique Fédérale de Lausanne : kathryn.hess@epfl.ch

Martin Hyland, University of Cambridge: M.Hyland@dpmms . cam.ac .uk

P. T. Johnstone, University of Cambridge: ptj@dpmms.cam.ac.uk

Anders Kock, University of Aarhus: kock@imf .au.dk

Stephen Lack, Macquarie University: steve.lack@mq.edu.au

F. William Lawvere, State University of New York at Buffalo: wlawvere@buffalo.edu

Tom Leinster, University of Glasgow, Tom.Leinster@glasgow.ac.uk

Jean-Louis Loday, Université de Strasbourg: loday@math.u-strasbg.fr

Ieke Moerdijk, University of Utrecht: moerdijk@math.uu.nl

Susan Niefield, Union College: niefiels@union.edu

Robert Paré, Dalhousie University: pare@mathstat.dal.ca

Jiri Rosicky, Masaryk University: rosicky@math.muni.cz

Giuseppe Rosolini, Università di Genova: rosolini@disi.unige.it

Alex Simpson, University of Edinburgh: Alex.Simpson@ed.ac.uk

James Stasheff, University of North Carolina: jds@math.upenn.edu

Ross Street, Macquarie University: street@math.mq.edu.au

Walter Tholen, York University: tholen@mathstat.yorku.ca

Myles Tierney, Rutgers University: tierney@math.rutgers.edu

Robert F. C. Walters, University of Insubria: robert.walters@uninsubria.it

R. J. Wood, Dalhousie University: rjwood@mathstat.dal.ca 